Teaching Critical Thinking in Higher Education

Avoiding, Detecting, and Explaining

Bias in Reasoning 



\section{Teaching Critical Thinking in Higher Education}

Avoiding, Detecting, and Explaining

Bias in Reasoning 


\section{Leden beoordelingscommissie:}

Prof. dr. A. B. H. de Bruin

Prof. dr. J. Elen

Prof. dr. S. M. M. Loyens

Prof. dr. S. F. te Pas

Prof. dr. J. W. F. van Tartwijk

Cover design and layout:

Hakim Sugito

\section{Print:}

Ridderprint BV., Alblasserdam

ISBN: 978-94-6375-878-9

(C) 2020 Eva Janssen

The research reported in this dissertation was funded by the Netherlands Organization for Scientific Research (NWO) under project number 409-15-203. It was carried out in the context of the Dutch Interuniversity Centre for Educational Research (ICO).

All rights reserved. No part of this dissertation may be reproduced or transmitted in any form, by any means, electronic or mechanical, without the prior permission of the author, or where appropriate, of the publisher of the articles. 
Teaching Critical Thinking in Higher Education

Avoiding, Detecting,and Explaining

Bias in Reasoning

Kritisch denken doceren in het hoger onderwijs:

Bias in redeneren leren

vermijden, herkennen en vitleggen

(met een samenvatting in het Nederlands)

\section{Proefschrift}

ter verkrijging van de graad van doctor aan de Universiteit Utrecht op gezag van de rector magnificus, prof.dr. H. R. B. M. Kummeling, ingevolge het besluit van het college voor promoties in het openbaar te verdedigen op

vrijdag 13 november 2020 des middags te 2.30 uur

door

Eva Marieke Janssen

geboren op 24 oktober 1990

te Delft 
Promoter:

Prof. dr. T. van Gog Copromotoren:

Dr. M. T. Mainhard

Dr. P. P. J. L. Verkoeijen

Dr. A. E. G. Heijltjes 


\section{Contents}

Chapter $1 \quad$ Introduction

\section{PART 1 Teaching critical thinking}

Chapter 2 Identifying characteristics associated with higher education teachers' Cognitive Reflection Test performance and their attitudes towards teaching critical thinking

Chapter $3 \quad$ Training higher education teachers' critical thinking and attitudes towards teaching it

Chapter $4 \quad$ Training higher education teachers' ability to explain biases in students' reasoning

\section{PART 2 Cognitive mechanisms and dispositions underlying biased reasoning}

Chapter 5 Recognizing biased reasoning: Conflict detection during

Chapter 6 "You're wrong!": The impact of accuracy feedback on the bat-and-ball problem

Chapter $7 \quad$ Psychometric properties of the Actively Open-Minded

Thinking scale

Chapter $8 \quad$ Summary and discussion

Nederlandse samenvatting (summary in Dutch)

Supplementary materials

References

Dankwoord 


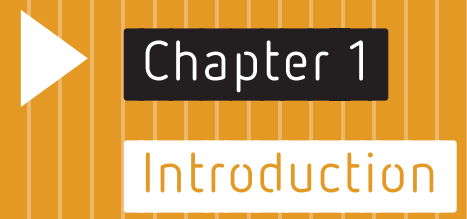


One of the major ambitions of higher education is to foster students' critical thinking (CT), to prepare them for functioning in a complex and rapidly changing society. Not only are CT-skills associated with higher levels of employment, a more sound financial situation, and stronger civic engagement (Arum et al., 2012), critically thinking citizens are considered to form the basis of a rational and democratic society (Facione, 1990a; Nussbaum, 2010). CT-skills, however, do not seem to develop automatically as a 'by-product' of higher education. Results of two large-scale longitudinal studies in the United States suggested that students' CT-skills hardly improved over the college years (Arum \& Roksa, 2011; Pascarella et al., 2011; although others are more optimistic Huber \& Kuncel, 2016). These findings are perhaps not surprising given that CT-skills are rarely taught explicitly (Jones, 2007). Yet, promoting $\mathrm{CT}$ in higher education requires $\mathrm{CT}$ to be an explicit educational goal at the institutional level, the teaching program level, and the course level (Elen et al., 2019).

According to a meta-analysis, the most effective intervention for improving students' CT-skills is a combination of authentic instruction, dialogue, and mentoring (Abrami et al., 2015). Because teachers are responsible for providing such interventions, they face a challenging task. Indeed, almost all empirical studies and theoretical papers on how to improve students' CT-skills point explicitly or implicitly to the crucial role of teachers in this process, and to the importance of teachers' own CT-skills (Abrami et al., 2008, 2015; Halpern, 1998; Pithers \& Soden, 2000; Ritchhart \& Perkins, 2005; Tishman et al., 1993). Nevertheless, there is a surprising paucity of research focusing on teachers and their teaching of CT. Therefore, the main aim of this dissertation was to start investigating how to equip higher education teachers ${ }^{1}$ with the knowledge and skills necessary for teaching one essential CT-skill: the ability to avoid bias in reasoning and decision-making (West et al., 2008).

\subsection{CRITICAL THINKING AND COGNITIVE BIASES}

It is still debated how CT should be defined, and conceptions of CT differ across disciplines. Philosophers tend to identify qualities of an ideal critical thinker (e.g., "criticality", Barnet et al., 2017), whereas psychologists tend to focus on cognitive actions critical thinkers should do when performing a task (e.g., "twelve key abilities of a critical thinker", Ennis, 1987). The educational perspective is closely tied to the psychological perspective but has a stronger focus on learning, teaching, and assessing CT. An important definition for purposes of educational assessment and instruction was formed by a Delphi panel of 46 experts from different fields (e.g., philosophy, psychology, education, physics, economics): "We understand critical thinking to be purposeful, self-regulatory judgment which results in interpretation, analysis, evaluation, and inference, as well as explanation of the evidential, conceptual, methodological, criteriological, or contextual considerations upon which that judgment is

\footnotetext{
${ }^{1}$ In this dissertation, the term "higher education teachers" refers to post-secondary teachers employed at a university (of applied sciences).
} 


\section{CHAPTER 1}

based" (Facione, 1990a). What this and many other definitions have in common is that CT is seen as complex higher-order thinking, which requires a large set of different cognitive skills (e.g., interpretation, analysis, evaluation) and dispositions (e.g., inquisitiveness, open-mindedness, flexibility). The complexity and broadness of the construct is also what makes it challenging to study CT in educational settings. It may not be realistic to expect one intervention to foster all aspects of CT or to measure the full construct with one test. It is, however, possible to zoom in on particular aspects of CT. In this dissertation I focused on a specific, yet, important aspect of $\mathrm{CT}$, namely, the ability to avoid bias in reasoning and decision-making (West et al., 2008).

In the CT literature, scholars have viewed the ability to evaluate evidence and arguments independently of one's prior beliefs and opinions as an important aspect of CT (Baron, 2008; Ennis, 1987; Perkins et al., 2000; Sternberg, 2001). This is also illustrated in tests that measure $\mathrm{CT}$, in which an important component is assessing the ability to avoid reasoning that is biased by prior opinion and prior belief (Ennis et al., 1985; Facione, 1990b; West et al., 2008). In addition, cognitive theorists have analyzed CT in terms of rational thinking concepts and the philosophy of rational thought (Siegel, 1988). Biases - systematic errors in people's thinking - violate the normative rules of rationality as set for instance by logic or probability (Stanovich et al., 2016; Tversky \& Kahneman, 1974). For example, due to the base-rate neglect bias (Kahneman \& Tversky, 1973), most people are more concerned about the risks of terrorism than about statistically more likely risks that they confront in daily life (e.g., a car accident; Sunstein, 2003). Other examples of biases that commonly occur are that people tend to look for confirmation instead of falsification when testing hypotheses (Wason, 1968), tend to infer conclusions based on personal beliefs in violation of logic (Evans et al., 2001), and tend to make choices that are affected by irrelevant contextual information (Jacowitz \& Kahneman, 1995; Tversky \& Kahneman, 1986). Biases are caused by intuitive or "heuristic" thinking. Heuristics are mental short-cuts that help to make quick decisions without loading heavily on the limited working-memory capacity. For example, due to the availability heuristic - which relies on immediate examples that come to a person's mind people automatically realize that there is high chance of traffic jam during rush hour (i.e., they can easily think of previous examples of rush hour traffic jams). This same availability heuristic, however, also opens the door to the base-rate neglect bias in risk perception of terrorism (i.e., people can easily think of previous terror attacks and therefore consider the chance that it will happen again relatively high). Although heuristics are indispensable for efficient reasoning, and biases are inherent to human cognition and often relatively innocent, there are many situations in which biased decisions can have serious consequences. For example, when a judge misinterprets evidence based on intuitive stereotypical associations (Eberhardt et al., 2006; Thompson \& Schumann, 1987), when a doctor makes a diagnostic error due to the exposure of media-provided information about a disease (Schmidt et al., 2014), when investors make bad investment decisions based on the mere familiarity of a stock (Oster \& Koesterich, 2013), or when parents decide not to vaccinate their children because of remembering rare instances in which vaccines have failed (Smith, 2017).

To assess biases in thinking, researchers have designed heuristics-and-biases tasks. These are reasoning problems in which an intuitively cued or heuristic response conflicts 
with elementary rational principles (Tversky \& Kahneman, 1974), consider the following (classical) example:

Premise 1: All living things need water.

Premise 2: $\quad$ Roses need water.

Conclusion: Roses are living things.

Does the conclusion follow necessarily from the premises?

Most reasoners intuitively conclude that the conclusion follows necessarily from the premises. However, after some reflection, it should become clear that the correct answer requires you to replace your intuitive or heuristic response ("yes, of course roses are living things") with a logical response ("the first premise does not necessarily imply that all things in need of water are indeed living things"). Although the logical answer does not require strong logical reasoning skills, numerous studies have shown that even educated reasoners fail to solve the problem correctly, with correct solution rates of approximately $30 \%$ (e.g., Markovits \& Nantel, 1989; Sá et al., 1999). Correct performance on heuristics-and-biases tasks has been positively associated with a person's ability to make unbiased judgments and decisions in a wide variety of (real-world) contexts, also after controlling for explained variance due to cognitive ability (Pennycook, Cheyne, et al., 2015; Pennycook, Fugelsang, et al., 2015b; Primi et al., 2016; Toplak et al., 2011, 2014, 2017).

\subsection{COGNITIVE MECHANISMS AND DISPOSITIONS UNDERLYING BIASED REASONING}

Although it is well established that our thinking is often biased, the precise nature of this bias is less clear. Researchers have explained (in)correct performance on heuristics-and-biases tasks with individual differences in people's thinking dispositions, their available mindware, and their cognitive ability (Campitelli \& Gerrans, 2014; Frederick, 2005; Heijltjes et al., 2015; Klaczynski, 2014; West et al., 2008). It is theorized that people with strong rational thinking dispositions - people with the tendency to enjoy and engage in effortful thinking (cf. Need for Cognition; Cacioppo \& Petty, 1982; Cohen et al., 1955) and in open-minded thinking (cf. Actively Open-minded Thinking; Baron, 1991; Stanovich \& West, 1997) - are more inclined to engage in further reflection. Consequently, they are more likely to detect a potential conflict between their heuristic response and other, more rational, considerations and to reason correctly (Stanovich et al., 2016; Stanovich \& West, 1998). However, merely detecting the need to think further is necessary but not sufficient for a good decision. One also needs to possess the requisite mindware, that is, the declarative knowledge and skills needed for the reasoning situation (e.g., knowledge of logical rules, statistical probabilities), and sufficient working memory capacity to start and sustain an override of the intuitive, heuristic response. Indeed, studies confirmed that providing learners instruction on relevant mindware was effective for improving performance on heuristics-and-biases tasks (e.g., Heijltjes, Van Gog, Leppink, et al., 2014; Van Peppen et al., 2018). Moreover, multiple studies showed that thinking dispositions, mindware, and cognitive ability were independent predictors of 


\section{CHAPTER 1}

successful performance on such tasks (Campitelli \& Gerrans, 2014; Toplak et al., 2011, 2014; West et al., 2008).

Until recently, influential scholars in the field suggested that most people perform poorly on heuristics-and-biases tasks because they do not recognize that their intuitive heuristic response is at conflict with logical or probabilistic principles; it was assumed that biased reasoners are completely unaware of the error in their reasoning (Evans \& Stanovich, 2013; Kahneman, 2011). Hence, people do not necessarily lack the requisite mindware or cognitive ability, but reason based on intuition without recognizing the need to engage in more effortful thinking (i.e., no conflict detection). Vice versa, it was suggested that the few people who do not respond biased to a reasoning problem were able to override their intuitive response and corrected it during more effortful thinking. Interestingly, however, recent studies have started to show that, even though they make a biased decision, most biased reasoners do show at least some sensitivity to the conflict between their heuristic response and the normative considerations. This is, for example, indicated by decreased confidence and longer response times (De Neys, 2014; Frey et al., 2018; Pennycook, Fugelsang, et al., 2015a). In addition, two studies showed that for most unbiased reasoners (i.e., correct performers) their intuitive response was already the correct, unbiased, response (Bago \& De Neys, 2017, 2019).

Thus, it is known that people's thinking dispositions, their available mindware, and their cognitive ability are important predictors of performance on heuristics-and-biases tasks. However, the precise cognitive mechanisms underlying biased reasoning are still debated. Acquiring further insight into the nature of biased reasoning is important because it can ultimately inform us on what works and why when teaching students to avoid bias in their thinking.

\subsection{TEACHING CRITICAL THINKING}

Most research on training to avoid biased reasoning and on teaching CT in general, is focused on students and on how to improve their thinking skills. Only a few studies focused on teachers and their teaching of CT. Nevertheless, based on findings about what works for students, one can infer what qualities teachers should possess to effectively teach CT. In addition, the little CT research with teachers and the general research base on teaching and teacher education offer some directions about what teachers may need to be able to teach CT.

As for findings on what works for improving students' CT-skills, results of meta-analyses showed that the most effective CT-intervention for student outcomes was a combination of authentic instruction, dialogue, and mentoring (Abrami et al., 2015) and that the most effective pedagogical grounding of the CT-intervention was achieved when instructors received special advanced training in preparation for teaching CT-skills (Abrami et al., 2008). Regarding the specific skill to avoid bias in reasoning, experimental studies with students have shown that explicit instruction about cognitive biases (through a video or a text) combined with the opportunity to practice with CT-tasks addressing these same biases improved students' performance on such tasks compared to a control condition, both on an immediate posttest (Heijltjes et al., 2015; Heijltjes, Van Gog, \& Paas, 2014) and on a delayed posttest two or three weeks later (Heijltjes, Van Gog, Leppink, et al., 2014; Van 
Peppen et al., 2018). A problem with all CT-interventions (both on avoiding bias and on CT-skills in general), however, is that the effects hardly seem to transfer across tasks or contexts (Heijltjes et al., 2015; Heijltjes, Van Gog, Leppink, et al., 2014, 2014; Kenyon \& Beaulac, 2014; Ritchhart \& Perkins, 2005). A lack of transfer across contexts is problematic because the ultimate goal of CT teaching is that students can apply the learned CT-skills outside the school. Empirical evidence on how to achieve transfer is scarce, especially with regard to the skill to avoid biased reasoning. Although there is not much empirical evidence available, it has been argued that to achieve transfer, thinking skills need to be taught with many different types of (real-world) examples and corrective feedback, while highlighting the underlying principles, so that students learn to recognize when and what thinking skill is needed in a particular situation (Halpern, 1998; Ritchhart \& Perkins, 2005; Tishman et al., 1993).

Thus, teachers need to be very skilled in avoiding bias in their own reasoning if they want to teach students CT-skills that help them to avoid bias in their reasoning and to achieve transfer. Teachers need to be able to provide explicit instruction on important reasoning biases and be able to integrate practice with avoiding biased reasoning in their lessons (e.g., through dialogues, mentoring and feedback on students' reasoning). To achieve this, knowing how to avoid biased reasoning oneself is a necessary condition. However, it is not sufficient: for teachers to be able to engage their students in dialogue about biases and to provide them adequate feedback, they also need the ability to detect the biases in their students' reasoning and to clearly explain to them why their reasoning is biased. The few available studies indicate that teachers may not always have a concrete understanding of what CT encompasses or how they can teach it explicitly (Choy \& Cheah, 2009; Dumitru, 2018; Morais et al., 2018; Paul et al., 1997; Stedman \& Adams, 2012). This would be problematic, given the importance of explicit instruction for effective CT teaching.

Moreover, a lack of CT-skills or a lack of concrete understanding on how to teach CT may also negatively affect motivation for teaching it (Bandura, 1982). More specifically, in line with expectancy value theory (Eccles \& Wigfield, 2002), research showed that teachers with a positive attitude towards the relevance of teaching a particular subject (i.e., high task value) and confidence in one's ability (i.e., high expectancy of success) were more likely to engage in effective teaching (Klassen \& Tze, 2014; Lorencová et al., 2019; Paul et al., 1997; Van Aalderen-Smeets \& Walma van der Molen, 2013, 2015). Although this has not yet been empirically studied in the domain of CT-teaching, an experimental study on science teaching showed that interventions can positively affect teaching attitudes: a training focused on changing primary school teachers' professional attitudes had a large effect on perceived relevance and self-efficacy beliefs regarding science teaching, as well as on self-reported teaching behavior (Van Aalderen-Smeets \& Walma van der Molen, 2015).

In conclusion, for being able to teach $\mathrm{CT}$, there are three important variables, which formed the central focus in this dissertation: (1) teachers' own CT-skills, operationalized as the ability to avoid bias in one's own reasoning and decision-making; (2) teachers' CT-teaching-skills, operationalized as the ability to detect and explain biases in students' reasoning; and (3) teachers' positive attitudes towards teaching CT, operationalized as perceived relevance of and perceived competence in teaching CT. 


\section{CHAPTER 1}

\subsection{CONTEXT AND OVERVIEW OF THIS DISSERTATION}

This dissertation is one of the outcomes of a larger project named "Investing in Thinking Pays Good Interest: Improving Critical Thinking Skills of Students and Teachers in Higher Professional Education", funded by the Netherlands Organization for Scientific Research and co-financed by Avans University of Applied Sciences (project number 409-15-203). In this project, researchers from Utrecht University and Erasmus University Rotterdam collaborated closely with educational advisors, teachers, and researchers from Avans University of Applied Sciences. The project consisted of two subprojects. The first was on how to improve and maintain students' CT-skills to avoid bias in their reasoning and to achieve transfer (conducted by Lara van Peppen, Erasmus University Rotterdam). The second subproject focused on how to equip teachers for teaching students to avoid biased reasoning and resulted in this dissertation (conducted by the author of this dissertation).

The first part of this dissertation (Chapters 2, 3, and 4) comprises three studies addressing the main question "How can higher education teachers be equipped with the knowledge and skills necessary for teaching critical thinking?". The second part (Chapters 5, 6, 7) contains three additional studies on the cognitive mechanisms (Chapters 5 and 6) and dispositions (Chapter 7) that underlie biased reasoning. Studying these mechanisms can contribute to our understanding of what might work and why when teaching to avoid biased reasoning. Finally, a summary and discussion of the main findings from the six studies is provided (Chapter 8), along with implications of the research findings and suggestions for future research.

\section{PART 1: TEACHING CRITICAL THINKING}

Knowing what variables play a role in higher education teachers' CT-skills and attitudes towards teaching CT can inform research on how to better equip teachers for teaching it. Thus, as a first step, the study in Chapter $\mathbf{2}$ investigated what teacher characteristics are associated with higher education teachers' CT-skills and their attitudes towards teaching CT. CT-skills were assessed with an expanded version of the Cognitive Reflection Test (CRT; Toplak et al. 2014a), which measures the ability to avoid biased reasoning on seven short math problems. Teaching attitudes were measured with a questionnaire that addressed teachers' perceived relevance of and perceived competence in teaching CT.

The study presented in Chapter 3 tested whether teachers' CT-skills, CT-teaching-skills, and teaching attitudes can be fostered through training. In a quasi-experimental study, I investigated the impact of a three-session teacher training. The first session provided explicit instruction on cognitive biases and opportunity to practice with tasks addressing the instructed biases. The second and third sessions focused on strengthening teachers' attitudes and skills towards teaching $\mathrm{CT}$ through discussing the relevance of teaching $\mathrm{CT}$, providing extra opportunity for practice, designing a domain-specific CT-task, and discussing ways to integrate CT during teaching. Prior to the start of the training, after the first session, and after the third (final) session, we measured teachers' CT-skills, CT-teaching-skills, and attitudes towards teaching CT. CT-skills were assessed with classical heuristics-and-bias tasks addressing teachers' ability to avoid the instructed reasoning biases (on learning tasks) and their ability to avoid related but non-instructed reasoning biases (on transfer tasks). 
CT-teaching-skills were assessed with vignette tasks, measuring teachers' ability to detect and explain biases in student papers. Teaching attitudes were again assessed with a questionnaire on teachers' perceived relevance of and perceived competence in teaching CT. Teachers in an untreated control condition completed the tests around the same points in time.

The study described in Chapter $\mathbf{4}$ focused on the crucial CT-teaching-skill of providing feedback on students' reasoning. It was investigated whether the ability to explain biases in students' reasoning would improve from training. In this experimental study, teachers in the control condition participated in a CT-training session on avoiding biases in their own reasoning (cf. first training session in Chapter 3) and teachers in the experimental condition additionally followed an individual online training on how to clearly explain biases in students' written arguments. It was investigated whether this explanation training would improve teachers' ability to explain reasoning biases in student papers immediately after the training and two weeks later (on both learning and transfer tasks). In addition, I again explored whether the training impacted perceived relevance of and perceived competence in teaching CT.

\section{PART 2: COGNITIVE MECHANISMS AND DISPOSITIONS UNDERLYING BIASED REASONING}

As noted in section 1.2, the precise cognitive mechanisms underlying biased reasoning are still debated. The study in Chapter 5 builds on recent research in which it has been shown that most biased reasoners seem somehow aware that their reasoning is incorrect; they show robust signs of error or "conflict" detection. One important shortcoming in this research, however, is that the conflict detection effect has only been studied with classical, arguably non-realistic, heuristics-and-biases tasks. Therefore, I investigated whether conflict detection also occurs during reasoning on longer, more complex, and more realistic tasks involving decision-making (problem solving) or decision-evaluation (paralleling a teacher's task to evaluate students' decisions in assignments).

The study in Chapter 6 explored people's reasoning on the popular bat-and-ball problem (one of the CRT items used in Chapter 2). The aim was to investigate the impact of minimal response feedback (i.e., telling participants whether their response is correct or incorrect; frequently done in educational settings) on people's task performance and conflict detection. To examine the nature of a potential learning effect, a two-response paradigm was adopted, in which participants first had to indicate their initial, intuitive hunch and then could take time to deliberate and change their answer.

The last study presented in Chapter 7, investigated the internal validity of the Actively Open-minded Thinking scale. This frequently used questionnaire intends to measure the disposition towards rational thinking (an important disposition for teachers to possess) and has been used to explain individual differences in biased reasoning. Yet, despite its frequent use, it was unclear whether and to what degree the items measure the psychological trait actively open-minded thinking. 


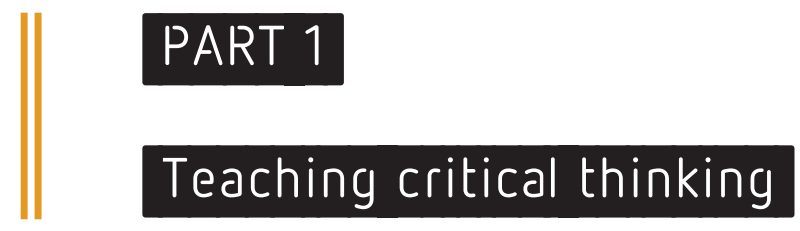





\section{Chapter 2}

\section{Identifying characteristics associated}

\section{with higher education teachers}

\section{Cognitive Reflection Test performance}

\section{and their attitudes towards teaching}

\section{critical thinking}

This chapter was published as:

Janssen, E. M., Meulendijks, W., Mainhard, T., Verkoeijen, P. P. J. L., Heijltjes, A. E. G., Van Peppen, L. M., \& Van Gog, T. (2019). Identifying characteristics associated with higher education teachers' Cognitive Reflection Test performance and their attitudes towards teaching critical thinking. Teaching and Teacher Education, 84, 139-149. doi:10.1016/j.tate.2019.05.008

\section{Acknowledgement of author contributions:}

EJ, WM, PV, AH, and TVG designed the study, WM, PV, and AH recruited participants and collected the data, EJ analyzed the data, TM checked the data package, EJ drafted the manuscript, all authors contributed to critical revision of the manuscript, TM, PV, AH, and TVG supervised the study. 


\section{ABSTRACT}

The aim of this study was to identify characteristics that are related to higher education teachers' $(N=263)$ Cognitive Reflection Test (CRT) performance, which assesses an important aspect of critical thinking (CT), and their attitudes towards teaching CT more generally. Results of a structural equation model showed that a stronger disposition towards effortful thinking, teaching in a more technological domain, and a higher level of education were related to a better CRT performance. Thinking dispositions were also related to teachers' perceived relevance of teaching CT. Confidence in CRT performance rather than actual performance was related to perceived competence in teaching CT.

Keywords. critical thinking; CRT; teaching attitudes; thinking dispositions; teaching and teacher education; higher education. 


\section{CHAPTER 2}

\subsection{INTRODUCTION}

The aim of this study was to identify characteristics that are related to higher education teachers' Cognitive Reflection Test (CRT) performance, which assesses an important aspect of critical thinking (CT), and their attitudes towards teaching CT more generally. Teaching $\mathrm{CT}$ is an important topic in higher education, since one of the major ambitions of higher education is to foster students' CT-skills (National Research Council, 2012). Teachers have an important role to play in this process, as it has been shown that students' CT-skills do not develop automatically as a by-product of higher education (Arum \& Roksa, 2011; Pascarella et al., 2011) and students need explicit instruction to improve their CT-skills (Abrami et al., 2015; Heijltjes et al., 2014). Remarkably, even though reviews on teaching CT highlight the crucial role of the teacher (Abrami et al., 2008, 2015; Pithers \& Soden, 2000; Ritchhart \& Perkins, 2005), studies on teachers' CT are scarce and mostly focused on preservice teachers. According to the few available studies, higher education teachers (i.e., postsecondary teachers) may not have a concrete understanding of what CT encompasses and how they can teach it (Choy \& Cheah, 2009; Stedman \& Adams, 2012). This would be problematic for teaching $\mathrm{CT}$, because two basic requirements for being able to teach a particular subject or skill are possessing the skill oneself (Hattie, 2003; Jones \& Moreland, 2003) and having a positive attitude towards teaching it (Klassen \& Tze, 2014; Van Aalderen-Smeets \& Walma van der Molen, 2013, 2015). Variables that play a role in higher education teachers' CT-skills and attitudes towards teaching CT have not yet been identified, yet knowledge on these variables can be can be informative for research on how to better equip teachers for teaching CT. Thus, as a first step, the present study investigated what teacher characteristics were associated with higher education teachers' CRT performance (as an important aspect of $\mathrm{CT}$ ) and with positive attitudes towards teaching $\mathrm{CT}$ more generally.

\subsubsection{CRITICAL THINKING AND THE COGNITIVE REFLECTION TEST}

An essential aspect of $\mathrm{CT}$ is to avoid bias in reasoning and decision-making (i.e., rational thinking). Bias is said to occur when a reasoning process results in a systematic deviation from a norm when choosing actions or estimating probabilities (Stanovich et al., 2016; Tversky \& Kahneman, 1974). Examples of biases that commonly occur are that people tend to make predictions based on their intuition without taking the probability of an outcome into account (Kahneman \& Tversky, 1973), tend to look for confirmation instead of falsification when testing hypotheses (Wason, 1968), tend to infer conclusions based on personal beliefs in violation of logic (Evans et al., 2001), and tend to make choices that are affected by irrelevant contextual information (Jacowitz \& Kahneman, 1995; Tversky \& Kahneman, 1986). Biases in reasoning can have serious consequences for decision-making in both daily life and complex professional environments (Lunn, 2013; Schmidt et al., 2014; Thompson \& Schumann, 1987; Toplak et al., 2017). Researchers have been studying bias empirically with 
reasoning problems in which an intuitively cued heuristic response conflicts with elementary logical principles (also called heuristics-and-biases tasks). One test that has been studied extensively in the reasoning and decision literature is Frederick's (2005) 3-item Cognitive Reflection Test (CRT). The most famous problem in this test is:

A bat and a ball together cost $\$ 1.10$. The bat costs $\$ 1$ more than the ball.

How much does the ball cost?

Most reasoners intuitively conclude that the ball must cost 10 cents $(\$ 1+\$ 0.10=\$ 1.10)$. However, this conclusion is incorrect ${ }^{1}$ because in this scenario the bat costs 90 cents more than the ball instead of $\$ 1$. After some reflection, it should become clear that the correct answer requires a different calculation leading to the conclusion that the ball costs 5 cents $(\$ 1.05+\$ 0.05=\$ 1.10)^{2}$. The logical answer of 5 cents does not require strong mathematical skills, yet a number of studies have showed that even educated reasoners fail to solve the problem correctly (Frederick, 2005; Toplak et al. 2014a). CRT items are designed to measure people's tendency to override their intuitive incorrect response and to engage in further reflection that leads to the correct response (Frederick, 2005). The original 3-item CRT and extended versions have been shown to reliably predict a person's ability to make unbiased judgments and decisions in a wide variety of contexts (Pennycook et al., 2015a; Primi et al., 2016; Toplak et al., 2011, 2014). In this study, we used the 7-item CRT developed by Toplak et al. (2014a) as a measure of an important aspect of CT.

Variables associated with CRT performance. In order to avoid a biased judgment on heuristics-and-biases tasks like for example the bat-and-ball problem, dual process theories explain that one needs to override an intuitive/heuristic Type 1 response $(\$ 1+\$ 0.10=$ $\$ 1.10)$ with a more effortful/logical Type $2(\$ 1.05+\$ 0.05=\$ 1.10$; Evans, 2008; Kahneman, 2011; Kahneman \& Frederick, 2005). Within the dual-process literature, researchers explain (in)correct performance on such tasks with individual differences in people's thinking dispositions, their available mindware, and their cognitive ability (Campitelli \& Gerrans, 2014; Frederick, 2005; Heijltjes et al., 2015; Klaczynski, 2014; West et al., 2008). People with strong rational thinking dispositions - people with the tendency to enjoy and engage in

\footnotetext{
${ }^{1}$ In research on reasoning, it is debated whether heuristic responses should be labelled "incorrect" or "biased" (for a review, see Stanovich \& West, 2000). For the sake of simplicity we use the terms "correct" response or "logical" response for the responses that are considered normatively correct following the rules of logic or probability and "incorrect" for responses that are not normatively correct according to the rules of logic or probability.

${ }^{2}$ The algebraic equation behind the problem is

$(x+1)+x=1.10$.

$2 x+1=1.10$

$2 x=0.10$

$\mathrm{x}=0.05$.
} 
effortful thinking and actively open-minded thinking - are more inclined to detect the need for a Type 1 override than people with less strong rational thinking dispositions (Stanovich, 2011; Stanovich et al., 2016). However, merely detecting the need to override is necessary but not sufficient for a good decision. One also needs to possess the requisite mindware, that is, the declarative knowledge and skills needed for the reasoning situation (e.g., in the example above, algebraic mathematical skills), and sufficient working memory capacity to start and sustain an override. All three variables are necessary conditions for being able to successfully perform the heuristics-and-biases tasks. For the CRT, numerous studies showed that thinking dispositions, numeracy mindware, and cognitive ability were independent predictors of successful performance (Campitelli \& Gerrans, 2014; Toplak et al., 2011, 2014). However, no research with teachers has been conducted yet. Knowledge on what teacher characteristics are related to a better CRT performance could provide some first insights into the variables that play a role in teachers' CT-skills (required for teaching CT). Based on the studies outlined above, we hypothesized that teachers with a stronger disposition towards effortful and actively open-minded thinking would have a better CRT performance (hypothesis 1a); that teachers from technological domains would perform better than teachers from economical and societal domains respectively, because the required numeracy mindware for the CRT is taught most explicitly in technological domains, followed by economics and society, respectively (hypothesis 1b); and that teachers with a higher level of education - associated with cognitive ability - would perform better on the CRT (hypothesis 1c).

\subsubsection{TEACHING ATITUDES}

In addition to the skill of thinking itself, believing that one is competent in teaching CT to students (perceived competence) and believing that teaching these skills is relevant (perceived relevance) may be positive antecedents of effective teaching. More general motivational theories, such as expectancy-value theory, frame these two factors in terms of expectancy for success (perceived competence) and task value (perceived relevance); both are viewed as direct predictors of task performance and persistence (Eccles \& Wigfield, 2002). In line with this framework, teaching research has indeed shown that teachers who have a positive attitude towards the relevance of teaching a subject (i.e., high task value) and confidence in their ability to do so (i.e., high expectancy of success) engage in more effective teaching (Klassen \& Tze, 2014; Van Aalderen-Smeets \& Walma van der Molen, 2013, 2015). For instance, studies in the domain of science teaching in primary school showed that these believes were positively related to joy in teaching science (Van Aalderen-Smeets \& Walma van der Molen, 2013) and that an intervention focused on changing teachers' professional attitudes on science education and personal attitudes towards science in general, positively affected both perceived relevance and competence, as well as self-reported science teaching behavior in the classroom (Van Aalderen-Smeets \& Walma van der Molen, 2015). The authors suggested that teachers' personal attitudes on science in general positively affect their professional attitudes towards teaching it. Given the importance of teaching attitudes for effective teaching, it is relevant to identify what teachers characteristics are related to 
positive attitudes towards teaching CT. However, it has not yet been investigated what characteristics are related to teachers' perceived relevance of and perceived competence in teaching CT. Based on findings by Van Aalderen-Smeets and Walma van der Molen (2015) in the domain of science, we hypothesized that teachers with a stronger disposition towards effortful and actively open-minded thinking - as an expression of personal attitude on CT in general - would perceive the teaching of CT as more relevant (hypothesis 2a). Additionally, given that performance attainment is one of the principal sources of expectancy and value (Bandura, 1982; Eccles \& Wigfield, 2002), we hypothesized that teachers with a better CRT performance would perceive the teaching of CT as more relevant (hypothesis $2 \mathrm{~b}$ ) and would perceive themselves as more competent in teaching it (hypothesis 3 ).

\subsubsection{THE PRESENT STUDY}

The aim of the present study was to identify teacher characteristics that play a role in three variables that we considered important for effectively teaching CT: (1) teachers' CRT performance (as important aspect of CT-skills); (2) teachers' perceived relevance of teaching CT (3) and teachers' perceived competence in teaching CT more generally. Based on the literature and hypotheses outlined above, we constructed one model testing all of our hypotheses (see Figure 2.1). In addition, we also tested whether CRT performance mediated the relationship between thinking dispositions and perceived relevance of teaching CT (because we hypothesized that thinking dispositions were positively associated with CRT performance and that, subsequently, CRT performance was positively associated with perceived relevance, see Figure 2.1).

Exploratory analyses. Potentially, teachers' calibration with regard to their CRT performance may also be relevant for their teaching attitudes and effective teaching behavior. Calibration reflects the degree to which individuals' judgments about their capability correspond to their actual capability (Lichtenstein, Fischhoff, \& Phillips, as cited in Alexander, 2013). A teacher with a realistic estimation of her CT-skills knows what CT-skills she already possesses and what skills need some extra practice before being able to teach them. An unrealistic estimation, however, may negatively affect teaching attitudes and behavior. For instance, a teacher with high ability in a particular CT-skill who judges his ability very low (under-confidence), may feel incompetent in teaching it and subsequently avoid teaching the skill to his students. Vice versa, a teacher with a low ability in a certain CT-skill who judges her ability very high (overconfidence), may feel very competent in teaching it but may teach the skill inadequately to her students. To gain insight in teachers' understanding of their own thinking skills, we used their confidence judgments regarding their CRT performance to explore how well they were calibrated and, additionally, whether these confidence judgments mediated our hypothesized relationship of CRT performance with perceived relevance of and perceived competence in teaching $\mathrm{CT}$. 


\section{CHAPTER 2}

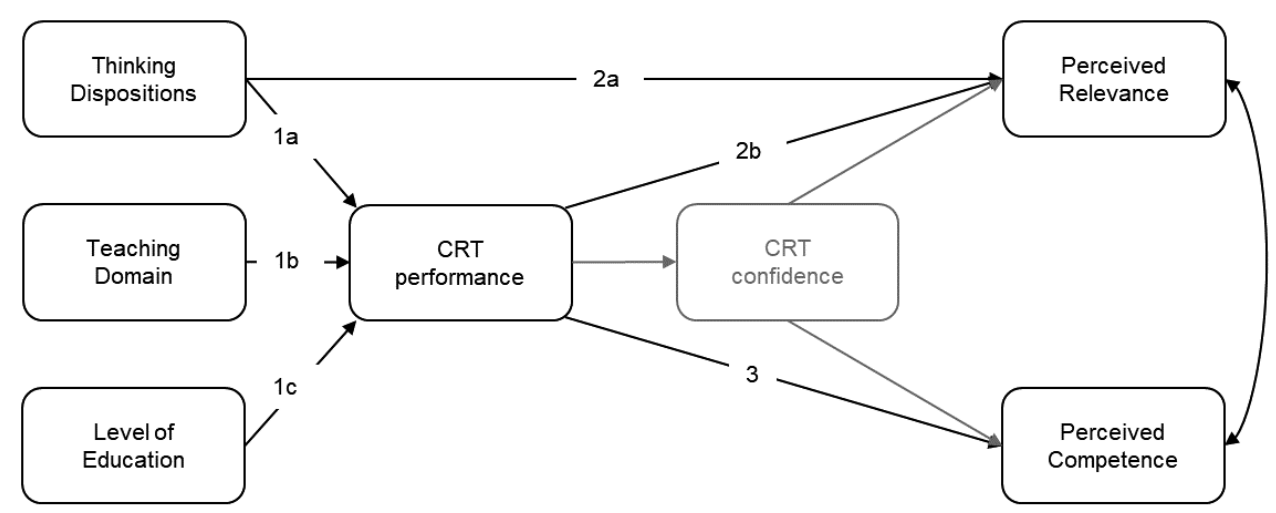

Figure 2.1. Hypothesized relationships between teachers' thinking dispositions, teaching domain, level of education, CRT performance, CRT confidence, Perceived Relevance, and Perceived Competence. Grey indicates exploratory analyses. CRT = Cognitive Reflection Test.

\subsection{METHOD}

\subsubsection{PARTICIPANTS}

Participants were teachers from a Dutch university of applied sciences. All 1378 teachers of this university received a request to participate via email. A total of 319 teachers started the questionnaire after providing informed consent. This response rate of $23.2 \%$ was about one standard deviation below the average of response rate of surveys in educational sectors (for a review, see Baruch \& Holtom, 2008), but could be expected because the announced survey was relatively long (i.e., 30 minutes), which negatively affects response rates (Galesic \& Bosnjak, 2009; Marcus et al., 2007). Additionally, we had to exclude data of 56 teachers: four because of noncompliance (e.g., answering with the same response to each question) and 52 because they already dropped out before completing the demographic questions or the CRT (i.e., the first task). Thus, self-selection bias seemed to play a role here; we return to this issue in the discussion. The final sample included 263 teachers $(41.4 \%$ female; age: $M=46.3$ years, $\mathrm{SD}=10.7$; teaching experience: $\mathrm{M}=9.8$ years, $\mathrm{SD}=8.7$ ).

\subsubsection{MATERIALS, PROCEDURE, AND DATA ANALYSIS}

We used an online survey with a forced response-format generated using Qualtrics Survey Software (Qualtrics, Provo, UT; http://www.qualtrics.com). The survey addressed four topics 
in a fixed order: (1) background variables, (2) CRT, (3) teaching attitudes, and (4) thinking dispositions. The survey was in Dutch for Dutch teachers and in English for non-Dutch teachers $(n=9)$.

Background variables. The demographic questions addressed Sex, Age (years), Teaching Experience (years), Level of Education, Teaching Domain, and CT-experience. Answer options for Level of Education ${ }^{3}$ were: bachelor/master program at a university of applied sciences $(n=54)$, bachelor/master program at an academic university $(n=182), \mathrm{PhD}(n=$ 26 ), and something else namely___ (one teacher reported vocational education as highest level of education). Answer options for Teaching Domain were (multiple answers possible): (1) technology; (2) ICT; (3) art \& design; (4) economics \& management; (5) welfare; (6) education; (7) health; and (8) law. We merged these into three broader domain-categories which paralleled the sections of the university of applied sciences: technology (category $1-3^{4}$ $n=96$ ), economics (category $4 ; n=102$ ), and society (category $5-8 ; n=65$ ). Some teachers taught in multiple domains (8\%): teachers in both technology and economics or society were assigned to the technological domain and teachers in both economics and society to the economical domain.

For CT-experience, teachers answered the question "Do you already have experience with CT? (multiple answers possible)". Answer options were: (1) I took a CT course/workshop at this University of Applied Sciences; (2) I took a CT course/workshop somewhere else, namely __ ; (3) I developed a CT course for students; (4) I taught a CT course for students; (5) I am a member of the CT community of this University of Applied sciences; (6) I read a book about CT, namely__ ; and (7) something else, namely__. We assigned one point for each reported activity. Two raters coded all open answers, which were rewarded with one point if the reported activity addressed CT explicitly and did not belong to one of the already listed categories. Absolute agreement between the two coders on the total CT-experience score was .997 (two-way random effects intraclass-correlation coefficient; see Shrout \& Fleiss, 1979). The two raters discussed the few inconsistent cases to reach consensus. The computed variable was a sumscore of the number of reported activities, one extra point was added to this sumscore if one of the reported activities involved - what we considered - deeper processing of CT-skills (i.e., teaching or developing a CT course, completing a bachelor/ master in philosophy, or conducting scientific research in the field CT). The possible score for CT-experience ranged from 0 to 8 , with higher scores representing a higher level of CT-experience.

\footnotetext{
${ }^{3}$ In the Dutch education system, higher education can be higher professional education offered by universities of applied sciences (Bachelor, Master), and academic education offered by academic universities (Bachelor, Master, PhD, with the $\mathrm{PhD}$ being an additional four-year trajectory after a Master degree).

${ }^{4}$ At the university of the present study, Art \& Design belongs to the technology domain because these subjects are embedded in a more technology/ICT context (e.g., programming).
} 
CRT. We measured CRT performance with a seven-item CRT developed by Toplak et al. (2014a), that we translated into Dutch. Toplak et al. (2014a) extended the original three-item CRT by Frederick (2005) to increase the reliability of the test and because the original items may have become familiar to participants. As explained (see section 2.1.1), the CRT is a short math test designed to measure the tendency to override an intuitive response-alternative that is incorrect and engage in further reflection to arrive at the correct response. An example item (Toplak et al. 2014a) is: "Jerry received both the 15th highest and the 15th lowest mark in the class. How many students are in the class? (intuitive answer: 30; correct answer: 29)". Six items had a free-response format and one item had a multiple-choice format with three answer options. Correct answers were rewarded with one point, incorrect answers with zero points. CRT performance was computed as the sumscore on all seven items and could therefore range from 0 to 7 . Cronbach's alpha in the original study was .72 and in our sample it was .66.

Confidence and calibration. Participants rated the confidence in their response to each CRT-item by answering the question "How certain are you that your response is correct?" on a four-point rating scale ranging from (1) very uncertain; (2) somewhat uncertain; (3) somewhat certain; to (4) completely certain. CRT confidence was the average of the confidence ratings for all seven CRT-items and could therefore range from 1 to 4 . To compute a calibration index, we identified the frequencies of the false negatives (i.e., very uncertain or somewhat uncertain judgment for a correct answer), true negatives (i.e., very uncertain or somewhat uncertain judgment for an incorrect answer), true positives (i.e., completely certain or somewhat certain judgment for a correct answer), and false positives (i.e., completely certain or somewhat certain for an incorrect answer) for each CRT-item separately.

Teaching attitudes. In order to measure teachers' attitudes towards teaching $\mathrm{CT}$ more generally, we constructed a questionnaire (see Table 2.1) that addressed teachers'(1) perceived relevance of teaching CT and perceived competence in teaching CT. Participants rated their agreement to 19 statements on a six-point rating scale ranging from (1) strongly disagree to (6) strongly agree. They received the following instruction "Suppose we would define critical thinking as follows: 'Critical thinking means that one engages in reflective reasoning before deciding what to believe or what to do, and that one can explain what those beliefs or decisions are based on'. Then to what extent do you agree with the following statements?" Thirteen of the 19 statements that followed were items from Stedman and Adams (2012; translated for the Dutch version), who measured perceptions of CT-instruction. Because the ability to avoid biases in reasoning and decision-making is an important element of CT, we added five items that addressed this element of $\mathrm{CT}$ as an addition to other $\mathrm{CT}$ elements that the items in the questionnaire addressed (see Table 2.1). We used one item as a control-item to check whether teachers adopted the frame of reference (see Table 2.1). Teachers should generally agree with this item if they had read the provided definition carefully, which was the case $(\mathrm{M}=4.8, \mathrm{SD}=1.0)$. Nine of the 18 items intended to measure Perceived Relevance; the other nine Perceived Competence. 
Because the questionnaire was a combination of self-constructed and not frequently used items, we examined the two-factor structure with a confirmatory factor analysis (CFA) using the 'Lavaan' package in R (R Development Core Team, 2008; Rosseel, 2012). We used a robust maximum likelihood (MLR) estimation as the assumption of multivariate normality was violated. Table 2.1 shows the standardized and unstandardized factor loadings. The model did not fit the data well, Comparative fit index $(\mathrm{CFI})=.85$, Root mean square error of approximation $(\mathrm{RMSEA})=.063$, and Standardized root mean square residual $(\mathrm{SRMR})=.074$. Based on the factor loadings in this model and reconsideration of the items' interpretation, we explored other factor structures. We found that a two-factor model with three items as indicators of Perceived Relevance and three of Perceived Competence (boldfaced items in Table 2.2) fitted the data well, CFI $=.98, \mathrm{RMSEA}=.054, \mathrm{SRMR}=.035$. The rationale behind the new item selection was that we selected only those items that addressed teachers' relevance perception of teaching CT for students' learning specifically, and only those that addressed teachers' perceived competence in teaching CT in one's courses. The excluded items of Perceived Relevance described other relevance aspects (i.e., relevance of CT in general, for enjoyment of learning, for active learning, or as personal responsibility to teach). The excluded items of Perceived Competence referred to a specific aspect of teaching CT (i.e., explaining or recognizing incorrect conclusions) or actually described behavior or faith in students' ability instead of competence perception. The thin lines in Figure 2.2 depict the final measurement model graphically, including the standardized factor loading and the squared multiple correlations (SMC). The SMSs indicate the (lower bound) reliability of the items. For example, Perceived Relevance accounted for $38 \%$ of the variance in y1. Item-correlations with means and standard deviations are shown in Table 2.2.

Thinking dispositions. Teachers' rational thinking dispositions were measured with two questionnaires: the 41-item Actively Open-minded Thinking scale (AOT; Stanovich \& West, 2007) and the 18-item (short form) of the Need For Cognition scale (NFC; Cacioppo et al., 1984). Again we used translations for the Dutch version of the survey (derived from Heijltjes et al., 2014). Participants rated their agreement to the 59 statements in total on a six-point rating scale ranging from (1) strongly disagree to (6) strongly agree. Scores on the items were averaged for NFC and AOT separately (after reverse scoring items that were formulated negatively) and could therefore range from 1 to 6 . Higher scores on the AOT represent a stronger tendency towards open-minded thinking. An example item is "A person should always consider new possibilities." Cronbach's alpha was .82 . Higher scores on the NFC represent a stronger tendency to engage in and enjoy thinking. An example item is "The notion of thinking abstractly is appealing to me." Cronbach's alpha was .85 . 


\section{CHAPTER 2}

Table 2.1. Standardized and unstandardized coefficients for the confirmatory factor analysis on the 18-item teaching attitudes questionnaire

\begin{tabular}{|c|c|c|c|c|}
\hline Observed variable & Construct & $\beta$ & $\mathrm{B}$ & SE \\
\hline CT is essential in making important decisions $(n)$ & PR & 0.38 & 1.00 & - \\
\hline $\begin{array}{l}\text { CT during educational activities discourages students from } \\
\text { active learning }(r)\end{array}$ & PR & -0.43 & -1.43 & 0.38 \\
\hline $\begin{array}{l}\text { CT allows students to better understand the course } \\
\text { content (y1) }\end{array}$ & PR & 0.63 & 1.46 & 0.33 \\
\hline $\begin{array}{l}\text { CT during educational activities encourages students to } \\
\text { become independent thinkers }\end{array}$ & PR & 0.58 & 1.35 & 0.28 \\
\hline $\begin{array}{l}\text { I believe it is more important for students to trust their intuition, } \\
\text { than to evaluate evidence }(r, n)\end{array}$ & PR & -.27 & -0.89 & 0.26 \\
\hline $\begin{array}{l}\text { Learning outcomes will not improve from CT during } \\
\text { educational activities }(r, y 2)\end{array}$ & PR & -.58 & -1.33 & 0.30 \\
\hline $\begin{array}{l}\text { I believe that it is my responsibility to promote } \mathrm{CT} \text { in my } \\
\text { courses }\end{array}$ & PR & .53 & 1.11 & 0.30 \\
\hline $\begin{array}{l}\text { CT is a way of thinking that would help students enjoy the } \\
\text { learning process }\end{array}$ & PR & 62 & 1.55 & 0.35 \\
\hline $\begin{array}{l}\text { СT helps students to see the difference between intuition } \\
\text { and a balanced argument }(y 3, n)\end{array}$ & PR & .53 & 0.98 & 0.23 \\
\hline While teaching, I look for specific evidence of CT by students & $\mathrm{PC}$ & 62 & 1.00 & \\
\hline If required, I could implement CT into my courses $(y 4)$ & PC & .72 & 1.06 & 0.13 \\
\hline $\begin{array}{l}\text { I think that students have barriers to CT, regardless of the } \\
\text { strategies I use (r) }\end{array}$ & $\mathrm{PC}$ & -.12 & -0.24 & 0.17 \\
\hline $\begin{array}{l}\text { In order for me to fully implement CT in my courses I would } \\
\text { need additional support }(r)\end{array}$ & $\mathrm{PC}$ & -.56 & -1.23 & 0.19 \\
\hline $\begin{array}{l}\text { I find it hard to explain to my students why they are drawing } \\
\text { incorrect conclusions from given information }(r, n)\end{array}$ & $\mathrm{PC}$ & -.37 & -0.75 & 0.19 \\
\hline $\begin{array}{l}\text { I have the skills necessary to promote students' CT in my } \\
\text { courses (y5) }\end{array}$ & $\mathrm{PC}$ & .60 & 1.03 & 0.16 \\
\hline $\begin{array}{l}\text { Usually, it is hard to determine whether students engage in CT } \\
\text { during my courses (reverse) }\end{array}$ & PC & -.58 & -1.22 & 0.18 \\
\hline $\begin{array}{l}\text { I am aware when students give intuitive answers during my } \\
\text { lessons (new). }\end{array}$ & $\mathrm{PC}$ & .26 & 0.41 & 0.13 \\
\hline $\begin{array}{l}\text { I find it hard to integrate } C T \text { in the content I am teaching ( } r \text {, } \\
\text { y6) }\end{array}$ & $\mathrm{PC}$ & -.69 & -1.48 & 0.22 \\
\hline $\begin{array}{l}\text { Critical thinking should always include a reflective component } \\
\text { (control item) }\end{array}$ & - & - & - & - \\
\hline
\end{tabular}

Note. $N=254$. CT $=$ critical thinking, $\mathrm{PR}=$ Perceived Relevance, $\mathrm{PC}=$ Perceived Competence, $\mathrm{r}=$ reverse, $\mathrm{n}=$ newly constructed items. Boldfaced variables are the indicators in the final measurement model in Figure 2.2. 
Table 2.2. Means (M), standard deviations (SD), and correlations between items measuring Perceived Relevance and Perceived Competence

\begin{tabular}{ccccccc}
\hline & $\mathrm{M}(\mathrm{SD})$ & $\mathrm{y} 1$ & $\mathrm{y} 2$ & $\mathrm{y} 3$ & $\mathrm{y} 4$ & $\mathrm{y} 5$ \\
\hline $\mathrm{y} 1$ & $4.89(0.82)$ & & & & & \\
$\mathrm{y} 2$ & $2.06(0.82)$ & $-.42^{\star \star \star}$ & & & & \\
$\mathrm{y} 3$ & $5.17(0.66)$ & $.35^{\star \star \star}$ & $-.32^{\star \star \star}$ & & & \\
$\mathrm{y} 4$ & $4.76(0.78)$ & $.36^{\star \star \star}$ & $-.20^{\star \star}$ & .12 & & \\
$\mathrm{y} 5$ & $4.30(0.91)$ & $.25^{\star \star \star}$ & -.11 & $.16^{\star}$ & $.49^{\star \star \star}$ & \\
$\mathrm{y} 6$ & $2.95(1.13)$ & $-.14^{\star}$ & $.16^{\star}$ & -.001 & $-.51^{\star \star \star}$ & $-.34^{\star \star \star}$ \\
\hline
\end{tabular}

Note. Range: $1-6 .{ }^{*} p<.05 .{ }^{\star \star} p<.01{ }^{\star \star \star} p<.001$.

\subsubsection{ANALYSIS}

To test our hypotheses, we computed a structural equation model (SEM) using the lavaan package in R (R Development Core Team, 2008; Rosseel, 2012). SEM is a combination of factor analysis (measurement model) and multiple regression (structural model). The measurement model examines relationship between the latent variables and their measures (i.e., answers to items). The structural model tests the interrelations among latent and observable variables. We included the measurement model of Perceived Relevance and Perceived Competence. We lacked power, however, to include the measurement models of all latent variables in our model; therefore, we included the latent variables that we measured with existing instruments (i.e., AOT, NFC, and CRT) as observed variables (mean centered). We used MLR estimation for our hypothesized model and a bootstrap estimation approach with 5000 samples to test the indirect effects in our explorative mediation analyses. Lastly, within our final sample of 263 teachers, 24 cases contained missing values: nine participants dropped out before starting the teaching attitudes questionnaire that followed after the CRT and, additionally, fifteen participants before starting the final questionnaire on thinking dispositions. Little's Missing Completely at Random (MCAR) test indicated that MCAR could be inferred, $\chi^{2}=32.91, d f=22, p=.063$. Therefore, we handled missingness using Full Information Maximum likelihood (FIML).

\subsection{RESULTS}

To check whether the required assumptions for SEM were met, we checked for normality, outliers, and linearity. First, CRT performance and CRT confidence were negatively skewed; therefore we reflected both variables (i.e., subtracting each score from the largest score plus 1) and subsequently applied square root transformations (Tabachnick \& Fidell, 2014). To enhance interpretation, we re-reflected the variables after transformation. Second, we identified a total of eight univariate outliers; two on CRT confidence, two on NFC, and four on AOT, that we winsorized to fit the distribution (i.e., the difference between the two 


\section{CHAPTER 2}

next highest or lowest values was added or subtracted to the next highest or lowest value with standardized value $<3.29$ or $>-3.29$; Tabachnick \& Fidell, 2014). We ran the analyses both with winsorized outliers and without outliers, yielding highly similar results (dissimilar results are reported). Third, in contrast to our hypotheses, there was no linear relationship between AOT and CRT; therefore this path was excluded from our structural model.

\subsubsection{DESCRIPTIVES}

Table 2.3 displays Spearman correlations between all study variables and the means with standard deviations. The teachers obtained, on average, relatively high scores on CRT performance, CRT confidence, Perceived Relevance, Perceived Competence, AOT, and NFC. Regarding calibration, Table 2.4 shows that $73.5 \%$ of all CRT performance judgments were accurate, indicating that teachers were quite well calibrated. Interestingly, accurate judgments mostly followed after a correct CRT performance (i.e. 1,218 out of the 1,354 accurate judgements concerned correctly performed CRT items), while the inaccurate judgments mostly followed after an incorrect CRT performance (i.e. 411 out of the 487 inaccurate judgments concerned in incorrectly performed).

The correlation analyses (Table 2.3) showed that CRT performance and CRT confidence were positively related $(r=.44, p<.001)$, indicating that teachers with a better CRT performance were on average more confident about their performance. Those with a higher confidence in their CRT performance were, however, not necessarily better performers: despite higher CRT confidence $(r=.31, p<.001)$, older teachers did not perform significantly better on the CRT than younger teachers (note, though, that more years of teaching experience was significantly positively related to CRT performance and confidence). Furthermore, teachers' need for cognition (NFC) was positively related to both their CRT performance $(r=.18$, $p=.005)$ and CRT confidence $(r=.22, p=.001)$, and also to their perceived relevance of teaching CT $(r=.34, p<.001)$ of and perceived competence in teaching CT $(r=.15, p$ $=.024$ ), and to amount of previous experience with CT-activities (CT-experience: $r=.15$, $p=.024$ ). Teachers' disposition towards actively open-minded thinking (AOT), was only significantly related to their perceived relevance of teaching CT $(r=.29, p<.001)$ and CT-experience $(r=.17, p=.008)$. 
Table 2.3. Means (M), standard deviations (SD), and spearman correlations between study variables

\begin{tabular}{lcccccccccc}
\hline & $\mathrm{M}(\mathrm{SD})$ & 1 & 2 & 3 & 4 & 5 & 6 & 7 & 8 & 9 \\
\hline 1. Sex & 0.41 & & & & & & & & & \\
2. Age & $46.3(10.7)$ & $-.27^{*}$ & & & & & & & & \\
3. Teaching exp. & $9.8(8.7)$ & -.08 & $.57^{*}$ & & & & & & & \\
4. CT exp. & $0.7(1.2)$ & .03 & .05 & .08 & & & & & & \\
5. CRT perf. & $4.9(1.8)$ & $-.17^{*}$ & .10 & $.17^{\star}$ & .05 & & & & & \\
6. CRT conf. & $3.5(0.6)$ & $-.27^{*}$ & $.31^{*}$ & $.24^{\star}$ & -.08 & $.44^{*}$ & & & & \\
7. PR & $5.0(0.6)$ & -.01 & .04 & -.05 & $.15^{\star}$ & .10 & .15 & & & \\
8. PC & $4.4(0.7)$ & -.06 & .07 & .09 & $.13^{\star}$ & .08 & $.17^{\star}$ & $.25^{*}$ & & \\
9. AOT & $4.7(0.4)$ & -.09 & .06 & -.07 & $.17^{\star}$ & .05 & .06 & $.29^{*}$ & .13 & \\
10. NFC & $4.6(0.5)$ & -.09 & .06 & -.07 & $.15^{\star}$ & $.18^{*}$ & $.22^{\star}$ & $.34^{*}$ & $.15^{\star}$ & $.35^{\star}$ \\
\hline
\end{tabular}

Note. Means and standard deviations are computed from the untransformed variables, correlation analyses included the transformed variables. Sex: male $=0$, female $=1 . C T=$ critical thinking, exp. $=$ experience, $\mathrm{CRT}=$ Cognitive Reflection Test, perf. $=$ performance. conf. $=$ confidence, $\mathrm{PR}=$ Perceived Relevance, $\mathrm{PC}=$ Perceived Competence, $\mathrm{NFC}=$ Need for Cognition, $\mathrm{AOT}=$ Actively Open-minded Thinking. Correlation analyses with excluded outliers instead of winsorized outliers did not yield any different results with regard to the direction or the significance of the correlations. ${ }^{*} p<.05$.

Table 2.4. Calibration index for the Cognitive Reflection Test (CRT)

\begin{tabular}{|c|c|c|c|c|c|c|}
\hline \multirow[t]{3}{*}{ Confidence judgment } & \multicolumn{6}{|c|}{ Performance } \\
\hline & \multicolumn{2}{|c|}{ Correct } & \multicolumn{2}{|c|}{ Incorrect } & \multicolumn{2}{|c|}{ Total } \\
\hline & $n$ & $\%$ & $n$ & $\%$ & $n$ & $\%$ \\
\hline \multirow[t]{2}{*}{ Very uncertain } & 24 & 1.3 & 48 & 2.6 & 72 & 3.9 \\
\hline & \multicolumn{2}{|c|}{ False negatives } & \multicolumn{2}{|c|}{ True negatives } & & \\
\hline Somewhat uncertain & 52 & 2.8 & 88 & 4.8 & 140 & 7.6 \\
\hline \multirow[t]{2}{*}{ Somewhat certain } & 310 & 16.8 & 186 & 10.1 & 496 & 26.9 \\
\hline & \multicolumn{2}{|c|}{ True positives } & \multicolumn{2}{|c|}{ False positives } & & \\
\hline Completely certain & 908 & 49.3 & 225 & 12.2 & 1133 & 61.5 \\
\hline Total & 1294 & 70.2 & 547 & 29.7 & 1841 & 99.9 \\
\hline
\end{tabular}




\subsubsection{STRUCTURAL EQUATION MODEL}

Figure 2.2 graphically displays the results of our SEM model including the thinking dispositions AOT and NFC, Teaching Domain (dummies), Level of Education (dummies), CRT performance, Perceived Relevance, and Perceived Competence. Our model fitted the data well, $\mathrm{CFI}=.94, \mathrm{RMSEA}=.041, \mathrm{SRMR}=.050$. The individual pathways are described below.

CRT performance. While taking the other predictors of the model into account, NFC was positively and significantly related to CRT performance $(\beta=0.14, \mathrm{SE}=0.07, p=.037$, $95 \% \mathrm{CI}=0.009,0.278)^{5}$. This indicated that teachers with a stronger disposition towards effortful thinking, achieved (on average) a higher CRT performance, which was in line with our hypothesis 1a. Furthermore, both dummy variables of Teaching Domain were significantly related to CRT performance (Economics vs. Technology: $\beta=0.14, \mathrm{SE}=0.07$, $p=.027,95 \% \mathrm{CI}=0.016,0.267$; Economics vs. Society: $\beta=-0.16, \mathrm{SE}=0.08, p=.016$, $95 \% \mathrm{CI}=-0.289,-0.030$ ), such that, as expected (hypothesis $1 \mathrm{~b}$ ), teachers in the domain of technology achieved the highest average score $(\mathrm{M}=5.49, \mathrm{SD}=1.42)$, followed by teachers in the domain of economics $(\mathrm{M}=4.84, \mathrm{SD}=1.89)$ and teachers in the domain of society $(\mathrm{M}$ $=4.20, \mathrm{SD}=1.81)$. Finally, the dummy variables of Level of Education were significantly related to CRT as well (Bachelor/Master at an Academic university vs. University of applied sciences: $\beta=-0.14, \mathrm{SE}=0.07, p=.017,95 \% \mathrm{CI}=-0.255,-0.025$; Bachelor/Master at an Academic university vs. PhD: $\beta=0.13, \mathrm{SE}=0.08, p=.004,95 \% \mathrm{CI}=0.041,0.221$ ), revealing that teachers with a $\mathrm{PhD}$ scored, on average, the highest $(\mathrm{M}=6.08, \mathrm{SD}=.1 .09)$, followed by teachers with an academic Bachelor or Master degree $(\mathrm{M}=4.91, \mathrm{SD}=1.80)$ and teachers with an applied-university degree $(\mathrm{M}=4.39, \mathrm{SD}=1.72)$, which was in line with our hypothesis $1 \mathrm{c} . \mathrm{R}^{2}$ indicated that the predictors in the model explained $16 \%$ of the variability in teachers' CRT performance, which is a medium overall effect (Cohen, 1988).

Perceived Relevance. A positive covariance between Perceived Relevance and Perceived Competence $(\beta=0.33, \mathrm{SE}=0.04, p=.019,95 \% \mathrm{CI}=0.024,0.273)$ showed that both teaching attitudes were moderately interrelated. Furthermore, the significant regression coefficients for both NFC $(\beta=0.33, \mathrm{SE}=0.08, p<.001,95 \% \mathrm{CI}=0.103,0.299)$ and AOT $(\beta=0.22, \mathrm{SE}$ $=0.14, p=.018,95 \% \mathrm{CI}=0.023,0.240)^{6}$ indicated that teachers with stronger dispositions towards effortful and actively open-minded thinking indeed perceived teaching CT as more relevant (hypothesis 2a). CRT performance was positively but not significantly related to Perceived Relevance $(\beta=0.11, \mathrm{SE}=0.09, p=.249,95 \% \mathrm{CI}=-0.045,0.172)$. Thus, in contrast to our hypothesis $2 \mathrm{~b}$, teachers with higher CRT performance did not perceive

\footnotetext{
${ }^{5}$ This path became nonsignificant in the analyses where we excluded the outliers: $\beta=0.13, \mathrm{SE}=0.07, p=.050,95 \%$ $\mathrm{Cl}=0.000,0.268$.

${ }^{6}$ This path became nonsignificant in the analyses where we excluded the outliers: $\beta=0.18, \mathrm{SE}=0.15, \mathrm{p}=.053,95 \%$ $\mathrm{Cl}=-0.001,0.215$.
} 


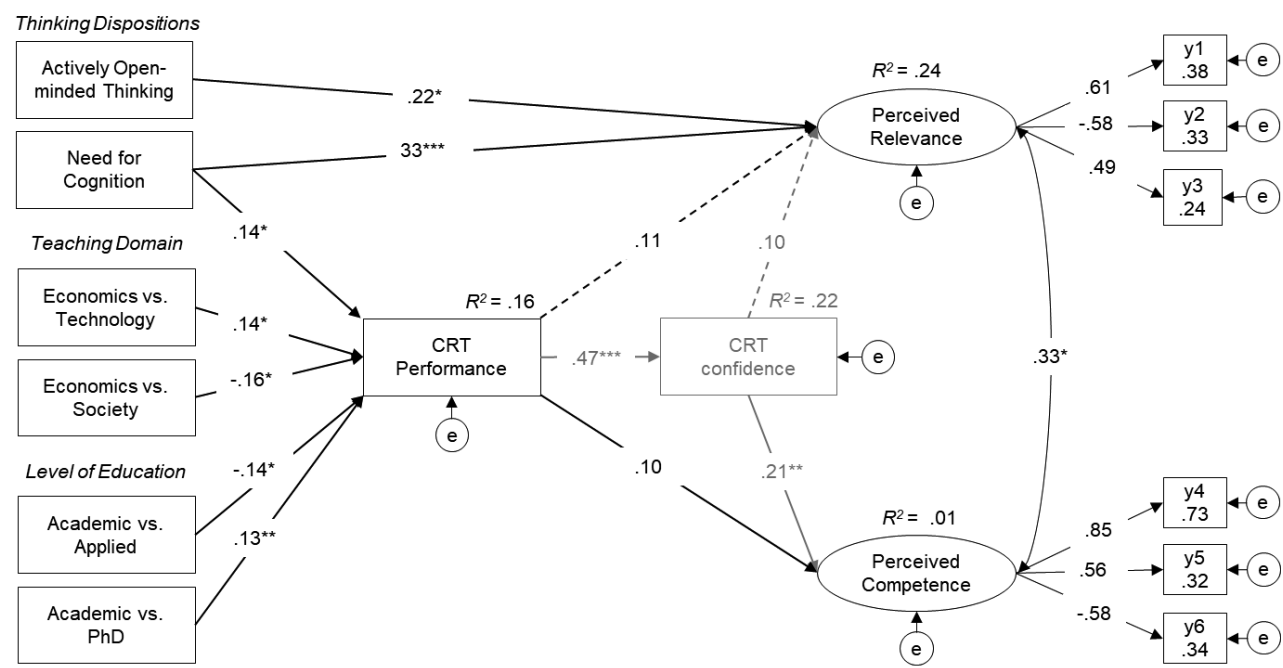

Figure 2.2. Results of the structural equation model of Thinking Dispositions, Teaching Domain (dummies), Level of Education (dummies), CRT performance, CRT confidence, Perceived Relevance and Perceived Competence. Rectangles and circles represent observed and latent variables, respectively. Values in $y 1$ to y6 are squared multiple correlations, indicating the reliability of each measure. Path coefficients are standardized regression weights. Dashed lines indicate nonsignificant paths. CRT = Cognitive Reflection Test. ${ }^{\star} p<.05 ;{ }^{* \star} p<.01,{ }^{\star \star \star} p<.001$

teaching CT as more relevant. $\mathrm{R}^{2}$ indicated that the predictors in the model explained $24 \%$ of the variability in teachers' perceived relevance of teaching CT, which is a large overall effect (Cohen, 1988).

Perceived Competence. Just as with Perceived Relevance, a positive but nonsignificant regression coefficient for CRT performance $(\beta=0.10, \mathrm{SE}=0.09, p=.139,95 \% \mathrm{CI}=$ $-0.028,0.200)$ suggested that teachers who scored higher on the CRT did not perceive themselves as more competent in teaching $\mathrm{CT}$, which was incongruent with our hypothesis 3. Not surprisingly, the predictors in the model explained almost no variance in teachers' competence perception towards teaching $\mathrm{CT}\left(\mathrm{R}^{2}=.01\right)$.

Mediation analyses. As mentioned in the introduction, we also tested whether CRT performance mediated the relationship between thinking dispositions and perceived relevance of teaching CT (see section 2.1.3). Additionally, we explored whether teachers' confidence in their CRT performance mediated the hypothesized relationship of CRT performance with Perceived Relevance and Perceived Competence (section 2.1.3). We conducted all mediation analyses in one explorative model (see grey paths in Figure 2.2) which yielded a good data fit as well, $\mathrm{CFI}=.94, \mathrm{RMSEA}=.039, \mathrm{SRMR}=.049$. Furthermore, the direction and significance of all previously described relationships remained the same. 


\section{CHAPTER 2}

First, we found no indirect effect of NFC via CRT performance on Perceived Relevance, $(\beta=0.01, \mathrm{SE}=0.01, p=.575,95 \% \mathrm{CI}=-0.012,0.027)$, suggesting that CRT performance did not mediate the relationship between teachers' disposition towards effortful thinking and their perceived relevance of teaching CT. Note, however, that the results of the first model already rejected our hypothesis that CRT performance was significantly related to Perceived Relevance.

Second, although we found a positive direct effect of CRT performance on CRT confidence $(\beta=0.47, \mathrm{SE}=0.01, p<.001,95 \% \mathrm{CI}=0.353,0.580)$, we found no direct effect of CRT confidence on Perceived Relevance $(\beta=0.10, \mathrm{SE}=0.55, p=.262,95 \% \mathrm{CI}=$ $-0.035,0.174)$, nor an indirect effect of CRT performance via CRT confidence on Perceived Relevance $(\beta=0.05, \mathrm{SE}=0.04, p=.252,95 \% \mathrm{CI}=-0.017,0.082)$. Hence, neither teachers' CRT performance nor their confidence in that performance was related to how relevant they perceived teaching $\mathrm{CT}$ to be. In contrast, CRT confidence did have a direct effect on Perceived Competence $(\beta=0.21, \mathrm{SE}=0.23, p=.004,95 \% \mathrm{CI}=0.067,0.307)$ and $\mathrm{CRT}$ performance had an indirect effect via CRT confidence on Perceived Competence $(\beta=0.10$, $\mathrm{SE}=.05, p=.007,95 \% \mathrm{CI}=0.030,0.149)$, indicating that - despite a non-significant overall effect of CRT performance on Perceived Competence - teachers who demonstrated better CRT performance were more confident about their CRT performance, and those teachers perceived themselves as more competent in teaching CT. Finally, the explained variance by the predictors in this explorative model remained more or less the same for CRT performance $\left(\mathrm{R}^{2}=.16\right)$ and Perceived Relevance $\left(\mathrm{R}^{2}=.25\right)$ as compared to our hypothesized model. For Perceived Competence, however it increased to $5 \%$ which is a small to medium effect (Cohen, 1988).

\subsection{DISCUSSION}

Research on CT highlights the crucial role of the teacher, yet research on teachers' CT and attitudes towards teaching it is scarce. This study was the first to investigate what teacher characteristics are associated with teachers' CRT performance, which assesses an important aspect of CT, and their attitudes towards teaching CT more generally. Our findings can inform future research on how to better equip higher education teachers for teaching CT.

\subsubsection{CRT PERFORMANCE}

As we hypothesized (Hypothesis 1a), teachers with a stronger disposition towards effortful thinking (NFC) indeed performed better on the CRT. In contrast, the disposition to engage in active open-minded thinking (AOT) was not related to CRT performance. Furthermore, it should be noted that the relationship between NFC and CRT was uncertain given the wide confidence interval for the regression coefficient and because its significance depended on including the (winsorized) outliers. Hence, we found no strong support for the hypothesized relationship between teachers' thinking dispositions and their CT-skills. This was surprising because previous studies in (mainly) student populations consistently showed that both NFC 
and AOT positively correlated with the CRT (Baron et al., 2015; Campitelli \& Gerrans, 2014; Frederick, 2005; Pennycook et al., 2015a; Szaszi et al., 2017; Thomson \& Oppenheimer, 2016; Toplak et al., 2011, 2014).

We see multiple possible explanations for these divergent findings. One explanation may be that we did not measure our latent constructs sufficiently. As we lacked power to test a full measurement model, we did not include NFC, AOT, and CRT as latent variables in our model (as we did with perceived relevance and perceived competence). Hence, the model did not test the data fit of these measures and did not take their measurement errors into account. This may be especially problematic for the AOT because - despite its frequent use and in contrast to the CRT and NFC - the factor structure is somewhat unclear. Svedholm-Häkkinen and Lindeman (2018) recently showed that AOT was not a unidimensional construct, which may be problematic for the interpretation of the sumscores. Future research should focus on further validating the AOT. Nevertheless, measurement problems do not provide an explanation of why other studies consistently found a relation between thinking dispositions and CRT performance as these studies also used sumscores instead of measurement models. Another potential explanation could be that this relationship does not apply to higher education teachers. However, a more likely explanation seems that our study sample was not representative. As participation was voluntary and the survey length relatively long, the teachers who finished the entire survey were probably very conscientious and/or already quite enthusiastic about CT. Hence, it is likely that we systematically over-sampled for teachers who had a stronger tendency to engage in and enjoy thinking and thus scored high on NFC. This self-selection bias could also have affected other variables in our study, since NFC has been shown to be a reliable predictor of performance on a wide range of CT-tasks and other thinking dispositions (Stanovich, 2011; Stanovich et al., 2016). Indeed, the teachers in our sample performed particularly well on the CRT and had relatively strong rational thinking dispositions: they scored more than thrice as high $(\mathrm{M}=4.9)$ on the CRT compared to a sample of Canadian university students $(\mathrm{M}=1.5 \mathrm{cf}$. Toplak et al. 2014a) and had stronger rational thinking dispositions (AOT: $\mathrm{M}=4.7 \mathrm{NFC}$ : $\mathrm{M}=4.6$ ) than students of that same university of applied sciences, as reported in another study (AOT M $=4.0$; NFC M $=3.9$ cf. Heijltjes et al., 2015). Moreover, the negatively skewed distributed CRT performance suggested that a considerable proportion of the teachers performed at ceiling, which may have caused a restricted range of values that reduced the correlations or made them more dependent on outliers in the sample.

Returning to our hypotheses, we expected that teaching in a technological domain would predict better CRT performance (Hypothesis 1b) than teaching in an economical or societal domain respectively, and this was indeed the case. Teaching domain was considered to reflect individual differences in available mindware, that is, the declarative knowledge and skills needed for correct reasoning. Our findings are in line with results of previous studies on mindware and CT (again mainly conducted with student populations) showing that numeracy skills, which play a more important role and are taught more explicitly in study programs in the technological domains, predicted performance on the CRT and other types of heuristics-and-biases tasks (Campitelli \& Gerrans, 2014; Frederick, 2005; Klaczynski, 2014; Liberali et al., 2012; Szaszi et al., 2017). 


\section{CHAPTER 2}

Level of Education was also related to CT-skills: academic teachers with a doctorate degree $(\mathrm{PhD})$ achieved the highest CRT average, followed by academic teachers without a doctorate, and non-academic teachers (University of Applied Sciences), respectively. As level of education is typically associated with cognitive ability, this corresponds with previous findings that cognitive ability predicts performance on the CRT and other heuristics-and-biases tasks (Frederick, 2005; Klaczynski, 2014; West et al., 2008).

In addition, we explored how well teachers were calibrated, that is, how accurately they could judge their own CRT performance. The calibration index showed that most teachers were, overall, highly accurate in judging their CRT performance. This is perhaps not surprising, because teachers in our study also performed well on the CRT, and it were the correct performances in particular that were judged accurately. That they were somewhat overconfident, is shown by the finding that inaccurate judgments mainly pertained to incorrect performances (rather than erroneously discarding a correct answer), indicating that teachers did not detect their thinking error when they made one. Our findings seem to confirm Hattie's (2013) suggestion (concerning students) that individuals know what they know, but are less able to judge what they do not know.

\subsubsection{TEACHING ATITUDES}

In line with our hypothesis $2 \mathrm{a}$, we found a relationship between teachers' thinking dispositions (both NFC and AOT) and perceived relevance. Hence, teachers' personal thinking dispositions indeed played a role in how relevant they perceived teaching CT to be. This finding is in line with the proposition by Van Aalderen-Smeets and Walma van der Molen (2015) within the domain of science teaching, that personal attitudes (on science in general) positively influence professional attitudes (towards teaching it). In this light, thinking dispositions could also be seen as an expression of personal attitude on CT in general. However, just as with NFC and CRT, the relationship between AOT and perceived relevance was uncertain given the wide confidence interval for the regression coefficient and because its significance depended on including the (winsorized) outliers. Here, we also propose that self-selection bias or the measurement quality of the AOT-scale may explain the ambiguous relationship.

Rather surprisingly and in contrast to our hypotheses, teachers' CRT performance was not related to perceived relevance of (hypothesis $2 \mathrm{~b}$ ) or perceived competence in (hypothesis 3 ) teaching CT. In the literature, however, performance attainments are viewed as one of the principal sources of one's task value and competence judgments (Bandura, 1982; Eccles \& Wigfield, 2002). A possible explanation for the lack of predictive value here may be that the CRT is not a good indicator of performance attainment in teaching CT. With the CRT we assessed a specific aspect of CT (i.e., rational thinking within a mathematical context) and related it to teachers' perceived relevance of and perceived competence in teaching CT more in general. Given the positive correlation between previous CT-experience and perceived competence, it is possible that positive experiences in following courses on CT or in teaching $\mathrm{CT}$ would be better predictors of perceived relevance of and perceived competence 
in teaching than a CRT performance. Therefore, it might be interesting for future research to investigate indicators of performance attainment in teaching CT. Another possible explanation for the nonsignificant relationship between CRT performance and perceived competence comes from our explorative mediation analyses, where we did find an indirect effect of CRT performance, via CRT confidence, on perceived competence. Hence, only those teachers with higher CRT scores and, subsequently, a higher confidence about that performance perceived themselves as more competent in teaching CT. This may imply that better CT-skills are related to higher perceived competence in teaching it, but only if one recognizes that one possesses the skill. This is in line with the expectancy value literature, which states that one's own interpretations of previous achievements are the antecedents of perceptions of competence (Eccles \& Wigfield, 2002). The additional direct effect of CRT confidence on perceived competence (independent of actual performance) suggests that teachers' competence perception may be affected by personal traits as well. Finally, note that the relatively small amount of explained variance in teachers' perceived competence also suggests that other characteristics not considered in our model (e.g., personality traits or positive experience with teaching $\mathrm{CT}$ ), may be more important.

\subsubsection{LIMITATIONS AND IMPLICATIONS FOR FUTURE RESEARCH}

The results of this study have to be seen in light of some limitations. First, given the self-selection bias that likely occurred, the results of this study should be interpreted with caution. We expect the means for CRT performance, thinking dispositions, and teaching attitudes in the general teacher population to be lower than observed in the current study. Furthermore, although we suspect that the self-selection bias probably reduced the size of the correlations in our study and that, consequently, the effect sizes for the studied relationships may be even larger in a more representative teacher sample (see section 2.4.1), we cannot know whether this is true. This is something that further research should point out. Nevertheless, most of our findings on the studied relationships were in line with previous findings in student populations, which seems to suggest they are meaningful.

A second potential limitation concerns our measurement instruments. We only focused on one (albeit important) aspect of CT (rational thinking) and regarded the CRT-score as a proxy of teachers' ability to avoid bias in reasoning and decision-making. Even though the CRT has been studied extensively and has been shown to be a reliable predictor of a person's ability to make unbiased judgments and rational decisions in a wide variety of contexts (Pennycook et al., 2015a; Primi et al., 2016; Toplak et al., 2011, 2014), it remains an open question whether our findings would also apply to other CT-tasks. Also note that the reliability of the CRT in our sample was somewhat lower than reported in the original study (in our study $\alpha=.66$ versus $\alpha=.72$ in Toplak et al. 2014a). We suspect that this low reliability can be explained by low variance, due to the very high CRT performance in our study. Furthermore, all of our instruments were Dutch translations of English questionnaires, and although we do not expect cultural differences to impact the findings, we cannot fully 


\section{CHAPTER 2}

rule this out, because a direct comparison has not been made. For the AOT and NFC, we used existing translations that had been used in previous research (Heijltjes et al., 2014) in which Dutch students achieved similar averaged sum scores on the translated questionnaires compared to a US student sample (Stanovich \& West, 2007) and, as in the US sample, their sum scores correlated positively with performance on heuristics-and-biases tasks. A final limitation is, as with any correlational design, that this study does not allow us to draw conclusions about causality or the directions of the studied relationships.

Despite these limitations, our findings have some interesting implications for future research on how to better equip higher education teachers for teaching CT. First, despite some ambiguous results, our findings underline that thinking dispositions are important to take into account when designing and investigating the effectiveness of interventions aimed towards improving teachers' CT-skills, attitudes, and ability to teach CT. An interesting open question for future research to address would be to what extent dispositions are malleable. For instance, the finding that CT-experience correlated positively with both dispositions (AOT and NFC) could imply that increasing experience with CT (e.g., through workshops, lectures, et cetera) would positively affect dispositions towards CT. On the other hand, teachers with stronger dispositions may have sought out more opportunities for engaging with $\mathrm{CT}$. If dispositions would be malleable, however, an important question would be whether changing a teacher's disposition towards effortful and actively open-minded thinking would lead to better performance on CT-tasks and a more positive teaching attitude, which together would ultimately affect teaching behavior and quality.

Second, our findings regarding teaching domain and level of education seem to endorse the important role of mindware and cognitive ability in CT-skills. Hence, when training teachers, it is important to take their mindware into account and to address potential knowledge gaps. In our study, numeracy was important. However, for other types of CT-tasks, different kinds of mindware may be required (e.g., rules of logic, probabilities, et cetera) and may need to be explicitly trained. Yet having mindware available does not guarantee its application, and dispositions may again be important here. Klaczynski (2014) found that only at high levels of thinking dispositions, numeracy mindware was predictive of performance on the CRT and four other types of heuristics-and-biases tasks. Put differently, it seems that possessing mindware (or possessing great cognitive capacity) is only beneficial when you are also favorably disposed towards thinking critically. Thus, when training teachers it can indeed be helpful to take mindware into account, but it is important to keep in mind that possessing relevant mindware is not equal to being less prone to biases in reasoning and decision-making. Finally, our finding that better CRT performance was not directly related to a more positive attitude towards teaching CT, may suggest that training teachers' CT-skills do not automatically reinforce their teaching attitudes. Therefore, future research should experimentally investigate the effects of CT-training on both teachers' CT-skills and their attitudes towards teaching it. 


\subsubsection{CONCLUSION}

In conclusion, by identifying variables that play a role in higher education teachers' CT-skills and teaching attitudes, the results of this study provide a first step towards future research on how to equip teachers for the important task of teaching CT. Future research should establish whether interventions targeting these variables would help to improve teachers' CT-skills, their teaching of CT and, ultimately, students' CT-skills. 


\section{Chapter 3}

Training higher education teachers'

\section{critical thinking and attitudes}

\section{towards teaching it}

This chapter was published as:

Janssen, E. M., Mainhard, T., Buisman, R. S. M., Verkoeijen, P. P. J. L., Heijltjes, A. E. G., Van Peppen, L. M., \& Van Gog, T. (2019). Training higher education teachers' critical thinking and attitudes towards teaching it. Contemporary Educational Psychology, 58, 310-322. doi:10.1016/j.cedpsych.2019.03.007

\section{Acknowledgement of author contributions:}

EJ, TM, PV, AH, and TvG designed the study, EJ, PV, and AH recruited participants and collected the data, EJ and RB analyzed the data, TM checked the data package, EJ drafted the manuscript, all authors contributed to critical revision of the manuscript, TM, PV, AH, and TVG supervised the study. 


\section{ABSTRACT}

Teachers play a crucial role in attaining a major objective of higher education: fostering students' critical thinking (CT). Yet, little is known about how to foster teachers' own CT-skills and attitudes towards teaching $\mathrm{CT}$. In a quasi-experimental study $(N=54)$, we investigated whether a three-session teacher training on (teaching) CT $(n=32)$ positively affected higher education teachers' CT-skills and their attitudes towards teaching CT compared to a control condition $(n=22)$. The training consisted of explicit instruction on common reasoning biases combined with assignments focused on the teaching practice. Results showed that the training improved teachers' performance on trained but not on novel CT-tasks. Also teachers' ability to detect biases in a written student product improved; however, despite a small improvement, they still had difficulties in correctly explaining those biases. Possibly due to ceiling effects the training did not affect perceived relevance of teaching CT. Finally, perceived competence in teaching CT decreased temporarily after the first training session but this negative effect disappeared after the final third session. Future research should investigate ways to promote teachers' ability to transfer trained skills to other CT-tasks, their ability provide feedback on students' reasoning (i.e., bias explanation), and their attitudes towards teaching CT.

Keywords. critical thinking; heuristics and biases; teaching and teacher education; instructional design, higher education. 


\subsection{INTRODUCTION}

One of the major ambitions of higher education is to foster students' critical thinking (CT) in order to prepare them for functioning in a complex and rapidly changing society. Indeed, CT-skills have been associated with higher levels of employment, a more sound financial situation, and stronger civic engagement (Arum et al., 2012; Toplak et al., 2017). CT-skills, however, do not develop automatically as a 'by-product' of higher education. Results of two large-scale longitudinal studies $(N=2322$ and $N=2212)$ in the United States, showed that students' CT-skills hardly improved over the college years (Arum \& Roksa, 2011; Pascarella et al., 2011). This is perhaps not surprising given that CT-skills are rarely explicitly taught (Jones, 2007), whereas research has shown that students need explicit instruction to improve their CT-skills (Abrami et al., 2015; Heijltjes, Van Gog, Leppink, et al., 2014). Because teachers are responsible for providing this explicit instruction, they play an important role in students' acquisition of CT-skills. Even though reviews on teaching CT have highlighted the crucial role of the teacher in this process (Abrami et al., 2008, 2015; Pithers \& Soden, 2000; Ritchhart \& Perkins, 2005) there is a paucity of research focusing on teachers' CT-skills. Moreover, the limited research that is available suggested that higher education teachers may not have a concrete understanding of what CT encompasses and how they can teach it (Choy \& Cheah, 2009; Stedman \& Adams, 2012). A prerequisite for being able to provide instruction and guidance to students on a subject is that teachers themselves possess the required skill, and that their attitude towards teaching it is positive: they need to perceive it as a highly relevant to teach and identify themselves as self-competent in teaching it (Eccles \& Wigfield, 2002; Klassen \& Tze, 2014; Watt \& Richardson, 2007; Zee \& Koomen, 2016). In the present study, we examined whether these preconditions can be positively affected through a training for higher education teachers (i.e., postsecondary teachers) consisting of explicit instruction on common reasoning biases combined with the opportunity for practice, along with assignments focused on their teaching practice.

\subsubsection{CRITICAL THINKING AND COGNITIVE BIASES}

In the CT literature, scholars have viewed the ability to evaluate evidence and arguments independently of one's prior beliefs and opinions as an important aspect of CT (Baron, 2008; Ennis, 1987; Perkins et al., 2000; Sternberg, 2001). This is also illustrated in tests that measure $\mathrm{CT}$, in which an important component consists of assessing the ability to avoid reasoning that is too biased by prior opinion and prior belief (Ennis et al., 1985; Facione, 1990b; West et al., 2008). In addition, cognitive theorists have analyzed CT in terms of rational thinking concepts and the philosophy of rational thought (Siegel, 1988). Biases violate the normative rules of rationality, as set, for instance, by logic or probability (Stanovich et al., 2016; Tversky \& Kahneman, 1974). For example, due to the base-rate neglect bias, most people are more concerned about the risks of terrorism than about statistically larger risks that they confront in daily life (Sunstein, 2003). Although biases are inherent to human cognition and often innocent, in some situations they lead to decisions that have serious consequences. For 


\section{TRAINING CRITICAL THINKING AND TEACHING ATIITUDES}

example, when a judge misinterprets statistical evidence (Thompson \& Schumann, 1987) or when a doctor makes a biased decision in medical diagnosis (Schmidt et al., 2014). In the present study we focus on this essential aspect of CT: the ability to avoid bias in reasoning and decision-making (i.e., rational thinking).

To assess biases in thinking, researchers have designed heuristics-and-biases tasks (Tversky \& Kahneman, 1974), consider for example the following (Frey et al., 2018):

In a study 1000 people were tested. Among the participants there were 5 sixteen-year-olds and 995 forty-year-olds. Lisa is a randomly chosen participant of the study. Lisa likes to listen to techno and electro music. She often wears tight sweaters and jeans. She loves to dance and has a small nose piercing.

What is most likely?

Lisa is sixteen

Lisa is forty

Because the description is very representative of a sixteen-year-old, a majority of the university students who were given this problem incorrectly indicated that Lisa is most likely sixteen. As explained by dual processing theories (Evans, 2008; Kahneman \& Frederick, 2005) one needs to replace a heuristic (Type 1) response "this description fits with the image of adolescents, Lisa is probably sixteen" with a more effortful logical (Type 2) response "there are also forty-year-olds that listen to techno wearing tight jeans, and since the study sample consisted of 995 forty-year-olds compared to only 5 sixteen-year-olds, it is most likely that Lisa is forty". Stanovich et al. (2016) argued that this shifting from a heuristic to a normative response requires a disposition towards rational thinking (e.g., actively open-minded thinking) and sufficient working memory capacity, but also what they referred to as 'mindware', that is, knowledge and skills needed for correct reasoning (e.g., of logic/ probability).

\subsubsection{TEACHING CRITICAL THINKING}

Research on the effectiveness of CT-instruction has mainly focused on students. The results of meta-analyses showed that the most effective type of CT-intervention for student outcomes was a combination of authentic instruction, dialogue, and mentoring (Abrami et al., 2015) and that the most effective pedagogical grounding of the CT-intervention was achieved when instructors received special advanced training in preparation for teaching CT-skills (Abrami et al., 2008). Regarding the specific skill to avoid biases in reasoning, experimental studies with students have shown that explicit instruction about cognitive biases (through a video or a text) combined with the opportunity for task practice improved students' performance on tasks addressing these same biases compared to a control condition, both at an immediate posttest (Heijltjes et al., 2015; Heijltjes, Van Gog, Leppink, et al., 2014; Heijltjes, Van Gog, $\&$ Paas, 2014) and on a delayed posttest two weeks later (Van Peppen et al., 2018). A problem 
with all CT-interventions (both on avoiding bias and on CT-skills in general), however, is that the effects hardly seem to transfer across tasks or contexts (Heijltjes et al., 2015; Heijltjes, Van Gog, Leppink, et al., 2014, 2014; Kenyon \& Beaulac, 2014; Ritchhart \& Perkins, 2005). A lack of transfer is problematic because the ultimate goal of CT-teaching is that students can apply the learned CT-skills in contexts outside the school context. Scholars argued that, to achieve transfer, thinking skills need to be taught with many different types of (real-world) examples and corrective feedback, while highlighting the underlying principles, so that students learn to recognize when and what thinking skill is needed in a particular situation (Halpern, 1998; Ritchhart \& Perkins, 2005). Hence, for students to achieve transfer, their teachers need to be very skilled in (teaching) CT, and able to bridge across various tasks and contexts.

In sum, teachers play an important role in students' CT-skills acquisition. In order to be able to teach CT, teachers first need to possess CT-skills themselves so that they can provide explicit instruction and integrate $\mathrm{CT}$ in their lessons (e.g., through dialogues, and feedback on students' reasoning). Based on the previous experiments on avoiding bias (Heijltjes et al., 2015; Heijltjes, Van Gog, Leppink, et al., 2014; Van Peppen et al., 2018), we expect explicit instruction of cognitive biases to improve teachers' performance on heuristics-and-biases tasks. However, as the previous experiments used student samples, it is an open question whether this indeed is the case. Moreover, for teachers to be able to engage their students in dialogue about biases and provide adequate feedback to their students, they also need to be able to detect biases in their students' reasoning and to explain errors students make. Finally, for teachers to apply CT-skills (acquired during the training) in practice, they need to have positive attitudes regarding CT-teaching. More specifically, in line with expectancy value theory (Eccles \& Wigfield, 2002), research showed that teachers with a positive attitude towards the relevance of teaching a particular subject (i.e., high task value) and confidence in one's ability (i.e., high expectancy of success) were more likely to engage in effective teaching (Choy \& Cheah, 2009, 2009; Klassen \& Tze, 2014; Paul et al., 1997; Van Aalderen-Smeets \& Walma van der Molen, 2013, 2015). Although this has not yet been investigated in the domain of CT-teaching, an experimental study in the domain of science teaching showed that interventions can positively affect teaching attitudes: a training focused on changing primary school teachers' professional attitudes had a large effect on perceived relevance and self-efficacy beliefs regarding science teaching as well as on self-reported teaching behavior (Van Aalderen-Smeets \& Walma van der Molen, 2015).

Thus, next to investigating whether training affects teachers' CT-skills as measured with heuristics-and-biases tasks, we also investigated whether teachers' ability to recognize and explain students' reasoning errors and their attitudes towards teaching CT improved.

\subsubsection{THE PRESENT STUDY AND HYPOTHESES}

The goal of the present study was to gain insight in how to equip higher education teachers with the skills and attitudes necessary for teaching CT. We explored the potential impact of a training consisting of three sessions of three hours each. The first session provided explicit instruction on cognitive biases and task practice (e.g., Heijltjes et al., 2015). The other two sessions focused on strengthening teachers' attitudes and skills towards teaching CT through 
discussing the relevance of teaching CT, providing extra opportunity for practice, designing a domain-specific CT-task, and discussing ways to integrate CT during teaching. Figure 3.1 displays an overview of the study design: we measured teachers' CT-skills and teaching attitudes before the start of the training, after its first session, and after its final third session (and at similar points in time in an untreated control condition). CT-skills were measured as (1) performance on heuristics-and-biases tasks, and (2) ability to detect and explain reasoning biases in a written student product (vignette).

First, we hypothesized that the explicit instruction on cognitive biases and the opportunity for task practice that the training provided, would positively affect teachers' performance on instructed tasks (i.e., learning; Hypothesis 1). We explored whether the training would lead to better performance on not-instructed tasks (transfer); although findings on effects of training on transfer were mixed so far, the generative learning activities provided in the additional sessions (e.g., designing a CT-task) may enhance transfer (Fiorella \& Mayer, 2016). Second, we expected that the training would positively affect teachers' ability to detect (hypothesis $2 \mathrm{a}$ ) and to explain (hypothesis $2 \mathrm{~b}$ ) reasoning biases in a student product. Third, regarding teaching attitudes, we expected (based on findings from science teaching, see previous section) that CT-instruction in combination with specific attention to the teaching practice would positively affect teachers' perceived relevance of (hypothesis 3a) and perceived competence in (3b) teaching CT.

To gain further insight in how the training affected teachers' task approach, we explored self-reported mental effort investment in relation to teachers' CT-task performance. Previous studies consistently found that instruction resulted in a more efficient way of solving the learning tasks, as indicated by a an improved task performance from pretest to posttest on instructed tasks without investing more mental effort in solving the tasks (Heijltjes et al., 2015; Heijltjes, Van Gog, Leppink, et al., 2014; Van Peppen et al., 2018). For transfer tasks, one study found no effects of instruction on invested mental effort (Heijltjes, Van Gog, Leppink, et al., 2014), whereas two other studies showed that - after instruction invested effort increased, yet without a performance improvement (Heijltjes et al., 2015; Van Peppen et al., 2018). This may indicate that training stimulated students to replace a heuristic (Type 1) response with a more effortful (Type 2) response to the new, not-instructed tasks (which would arguably be an important outcome of training), but that their mindware was not sufficiently automatized to correctly perform the task.

\begin{tabular}{|c|c|c|c|c|c|}
\hline Pretest & $\begin{array}{c}\text { Training } \\
\text { session } 1\end{array}$ & $\begin{array}{c}\text { Intermediate } \\
\text { test }\end{array}$ & $\begin{array}{l}\text { Training } \\
\text { session } 2\end{array}$ & $\begin{array}{l}\text { Training } \\
\text { session } 3\end{array}$ & Posttest \\
\hline $\begin{array}{l}\text { 1. Backgr. var. } \\
\text { 2. H-B tasks } \\
\text { 3. Det. \& expl. } \\
\text { 4. Teach. att. }\end{array}$ & $\begin{array}{l}\text { Bias instruction } \\
+ \text { task practice }\end{array}$ & $\begin{array}{l}\text { 1. H-B tasks } \\
\text { 2. Det. \& expl. } \\
\text { 3. Teach. att. }\end{array}$ & \multicolumn{2}{|c|}{$\begin{array}{l}\text { Retrieval practice }+ \text { assignments } \\
\text { focused on the teaching practice }\end{array}$} & $\begin{array}{l}\text { 1. H-B tasks } \\
\text { 2. Det. \& expl. } \\
\text { 3. Teach. att. }\end{array}$ \\
\hline
\end{tabular}

Figure 3.1. Overview of the study design. The control condition did not take part in the training sessions (dashed lines), only in the measurement occasions (solid lines). Backgr. var. = background variables; $\mathrm{H}$ $\mathrm{B}$ tasks = heuristics-and-biases tasks; Det. \& Expl. = Detecting and explaining reasoning biases in a student product; Teach. att. $=$ Attitudes towards teaching critical thinking. 


\subsection{METHOD}

\subsubsection{PARTICIPANTS AND DESIGN}

Participants were 56 teachers from a Dutch University of Applied Sciences ${ }^{1}$. Two participants were excluded (one was not proficient in Dutch and one was a policy maker instead of a teacher), leaving a final sample of 54 teachers $(63.0 \%$ female; age: $M=45.0$ years, $\mathrm{SD}=9.4$; teaching experience: $\mathrm{M}=8.4$ years, $\mathrm{SD}=6.7)$. The study had a quasi-experimental design, with a training condition $(n=32)$ and a control condition $(n=22)$. The teachers in the training condition voluntarily signed up for participation in the CT-training that consisted of three sessions of approximately three hours, spread over six weeks (the second session was three weeks after the first, the third session was two weeks after the second). Participation was recommended and endorsed by their faculty management. The training was given in three separate groups. Group A $(n=14)$ consisted of teachers from one department, teachers in Group B $(n=11)$ and $\mathrm{C}(n=7)$ came from different departments. The teachers in the control condition volunteered to take the tests and did not receive any training. The study consisted of six phases: (1) pretest; (2) first training session (3); intermediate test; (4) second training session; (5) third training session; (6) posttest. Teachers in the control condition only engaged in phase 1, 3, and 6 at approximately similar time intervals (see Figure 3.1).

\subsubsection{MATERIALS}

Pretest, intermediate test, and posttest. The tests were administered as an online survey with a forced response-format using Qualtrics Survey Software (Qualtrics, Provo, UT; http://www. qualtrics.com). The pretest addressed four topics in a fixed order: background variables, attitudes towards teaching $\mathrm{CT}$, bias detection and explanation in a student product, and heuristics-and-biases tasks. The intermediate test and posttest addressed the following outcome measures: teaching attitudes, bias detection and explanation, and heuristics-and-biases tasks.

Background variables. Because we were not able to randomly assign participants to conditions, we collected information on some background variables to check the comparability of the subsamples: gender, age (years), teaching experience (years), teaching domain, CT-experience, relevant experience, and thinking dispositions. Response categories for teaching domain were (1) technology/ICT; (2) economics/HRM/business administration; (3) social studies; (4) legal studies; other, namely _. These were merged into three broad domain-categories which paralleled the sections of the University of Applied Sciences: technology, economics, and society. For CT-experience, teachers answered the question "For how many hours (estimation) have you been actively involved with the theme 'thinking errors' in the past two years (think for example of following a workshop, teaching lessons, or developing lesson

${ }^{1}$ The Dutch education system distinguishes between higher education at an academic university (i.e., MSc at a research university) or non-academic university (i.e., university of applied sciences). 


\section{TRAINING CRITICAL THINKING AND TEACHING ATTITUDES}

materials)?" Answering categories were: 0 hours, 1-20 hours, 21-40 hours, 41-60 hours, 61-80 hours, 81-100 hours, or $>100$ hours. For relevant experience, teachers answered four yes/no questions that asked whether they had taught statistics, logic or programming and whether their job included the assessment of written student products.

Finally, we measured teachers' rational thinking dispositions with Dutch translations (Heijltjes, Van Gog, Leppink, et al., 2014) of two questionnaires: the 18-item short form of the Need For Cognition scale (NFC; Cacioppo et al., 1984) and the 41-item Actively Open-minded Thinking scale (AOT; Stanovich \& West, 2007). NFC intends to measure tendency to engage in and enjoy thinking and the AOT high-level epistemic goals and the tendency to reflect on rules of inference. Participants rated their agreement to the 59 statements in total on a six-point rating scale ranging from (1) strongly disagree to (6) strongly agree. Scores on the items were averaged for NFC and AOT separately (after reverse scoring items that were formulated negatively) and could therefore range from 1 to 6 . In the current study, the NFC had a Cronbach's alpha of .79 and the AOT of .78.

Heuristics-and-biases tasks. In line with previous research on the ability to avoid bias in reasoning and decision-making, we used several heuristics-and biases tasks as measures of CT (Heijltjes, Van Gog, Leppink, et al., 2014; Tversky \& Kahneman, 1974; West et al., 2008). Each test contained fifteen common heuristics-and-biases tasks (example items of all task categories are available in section 3.1. in the Supplementary Materials). All tasks at pretest, intermediate, and posttest were designed as structurally equivalent tasks but with different surface features. Two categories reflected learning tasks that were instructed and practiced during the training: (1) logic reasoning tasks in which belief bias played a role $(7 \mathrm{x})$, these tasks consisted of syllogisms that examined the tendency to be influenced by the believability of a conclusion when evaluating the logical validity of arguments and had a multiple-choice format with two answer options and one correct answer (adapted from Evans, 2002); and (2) tasks that assessed base-rate neglect in probability estimation $(3 \mathrm{x})$, which measure the tendency to overrate individual-case evidence (e.g., from personal experience, a single case, or prior beliefs) and to underrate statistical information. These tasks had multiple-choice formats with two, four, or six answer options where only a specific combination of selected answers was correct for the latter two (adapted from Fong et al., 1986; Stanovich et al., 2016; Stanovich \& West, 2000; Tversky \& Kahneman, 1974). We included a higher number of syllogisms because the chance of guessing the answer correctly was higher than for the base-rate tasks. Learning-task performance was computed as a percentage score to which both task categories contributed equally. Cronbach's alpha for the learning tasks was .37, and .43 on the intermediate test and posttest respectively.

Additionally, two task categories reflected transfer tasks (i.e., not addressed during the training): (1) tasks that assessed confirmation bias in logic reasoning (3x), which were Wason selection tasks that measure the tendency to verify logic rules rather than to falsify them, using a multiple-choice format with four answer options in which only a specific combination of two selected answers was correct (adapted from Evans, 2002; Gigerenzer \& Hug, 1992) and (2) tasks that assess covariation detection in probability estimation $(2 x)$, which measure the tendency to base estimations on already experienced evidence and disregard presented 
evidence (multiple-choice format with two answer options and one correct answer; adapted from Heijltjes, Van Gog, Leppink, et al., 2014; Stanovich \& West, 2000; Wasserman et al., 1990). Transfer-task performance was computed as a percentage score to which both task types contributed equally. Cronbach's alpha for the transfer tasks was .26 and .61, on the intermediate test, and delayed posttest, respectively.

After each task, participants reported their invested mental effort on a 9-point rating scale ranging from (1) very, very low effort to (9) very, very high effort (Paas, 1992).

Detecting and explaining reasoning biases. We constructed three vignettes - one for each measurement occasion - to assess teachers' recognition of reasoning biases in a written student product. Each vignette (about 600 words) was in the form of a summary of a bachelor thesis and contained five biases that were also addressed in the heuristics-and-biases tasks: belief bias $(2 \mathrm{x})$ and confirmation bias $(1 \mathrm{x})$ in logic reasoning and base-rate neglect $(1 \mathrm{x})$ and a covariation detection problem $(1 \mathrm{x})$ in probability estimation. Teachers were instructed to read the text carefully, to indicate all reasoning biases in the text, and to provide an explanation. Maximum score for each bias was two points, one point for detecting a reasoning bias (bias detection) and one point for a correct explanation (bias explanation). We pilot-tested one vignette and designed the other two as structurally equivalent but with the biases in a different order and different surface features. Bias detection and explanation were scored by two raters who coded $25 \%$ of the data. We found, respectively, substantial and almost perfect agreement (Landis \& Koch, 1977), ICC .63 and ICC $=.83$. The remainder of the data was scored by one rater (the first author). The final scores for bias detection and explanation were sumscores (range: 0-5 each).

Attitudes towards teaching. Attitudes towards teaching CT were measured with a questionnaire that originally consisted of 16 items, of which we selected 6 pretest-items (for details, see section 3.2. in the Supplementary Materials) that addressed teachers' perceived relevance of (3 items) and perceived competence in (3 items) teaching CT. The three items for relevance perception were a slightly adapted Dutch translation of items from Stedman and Adams (2012), who measured teachers' perceptions of CT instruction. An example-item is "Learning outcomes will improve from critical thinking during educational activities." The three competence perception items were constructed by the authors, an example item is "I can explain clearly to my students how they are drawing incorrect conclusions from the available information." Participants rated their agreement to the statements on a six-point rating scale ranging from (1) strongly disagree to (6) strongly agree. Averaged scores on the perceived relevance and perceived competence scales could therefore range from 1 to 6 . Per measurement occasion Cronbach's alpha for Perceived relevance was $.71, .75$, and .80 and for Perceived competence $80, .77$, and .70, respectively. A CFA showed that a two-factor model fitted the data well for each measurement occasion, $\chi^{2} \mathrm{~s}(8) \leq 9.81, p \mathrm{~s} \geq 0.279$, CFIs $\geq$ .97, TLIs $\geq .95$, RMSEAs $\leq .07$, SRMRs $\leq .06$. 


\section{TRAINING CRITICAL THINKING AND TEACHING ATTITUDES}

\subsubsection{TRAINING AND PROCEDURE}

The CT-training consisted of three sessions of approximately three hours, spread over six weeks. All sessions were given by the first author together with the fourth or fifth author. Two weeks before the start of the first training session of Group A (Group B started two days later; Group C two weeks later), all participants - both in the training and control condition - received a request via email to complete the pretest.

The first session provided explicit instruction combined with practice on CT-skills. The session consisted of four parts: (1) a general introduction on CT, heuristics and biases; (2) explicit instruction on belief bias in syllogistic reasoning and base-rate neglect in probability estimation (i.e., we presented multiple statements to which the teachers could respond whether or not the conclusions were correct according to them; hereafter we explained the correct answers using worked-examples); (3) the opportunity for practice on syllogistic reasoning problems; and (4) an intermediate test. During the last hour of the first training session, teachers in the training condition completed the intermediate test on their laptop in the same room. That same week, teachers in the control condition received a request via email to complete the intermediate test.

The second and third session focused on strengthening teachers' attitudes and skills towards teaching $\mathrm{CT}$ through providing extra opportunity for practice, discussing the relevance of teaching $\mathrm{CT}$, designing a domain-specific CT-task, and discussing ways to integrate CT during teaching. The second session consisted of (1) retrieval practice of the tasks from the first session; (2) an introduction on CT in education highlighting empirical findings on effective teaching strategies and learning outcomes from CT-instruction; and (3) a small-group assignment to design a CT task in the teachers' own teaching domain. The third session consisted of (1) discussion of the designed tasks; (2) discussing ways to integrate CT during teaching; (3) posttest; (4) evaluation. One hour of the third training session was again reserved for a posttest and teachers in the control condition again received a request via email to complete the posttest that same week.

\subsubsection{DATA ANALYSIS}

To examine how the training affected teachers' CT-skills and variables related to teaching it, we employed multilevel analyses for each outcome measure. Multilevel-analysis handled the $11 \%$ posttest drop-out (see below) without excluding cases list-wise (Hox, 2010). We used the 'lme4' package in R (Bates et al., 2015; R Development Core Team, 2008) and employed likelihood ratios to evaluate fit between a multilevel model and the data. For every outcome measure, we started with an intercept-only or so-called empty model (M1) including no predictors, that decomposed the variance of the outcome measure in two separate components: variance attributed to random error and to differences between the measurement occasions (level $1 ; \sigma_{e}^{2}$ ) and variance attributed to stable differences between the teachers (level $2 ; \sigma^{2}{ }_{u 0}$ ). We used this model to calculate the proportion of variance located at the teacher level, the intraclass correlation (ICC) $=\sigma_{u 0}^{2} /\left(\sigma_{u 0}^{2}+\sigma_{e}^{2}\right)$. Next, we tested an 
unconditional growth model with Occasion as fixed predictor (dummy-coded with the pretest as the reference category; M2), that tested a main effect of Occasion ${ }^{2}$. Third, we added a cross-level interaction between Occasion and Condition (with the control condition as the reference category; M3) which tested our hypotheses that teachers' progress on the outcome variables would differ from pretest to intermediate test and pretest to posttest, depending on being in the training or control condition. To compute effect sizes $\left(\mathrm{R}_{2}{ }_{2}\right)$, we used M2 as baseline model and calculated how much of the variance between teachers' scores could be explained by the predictors in the final model, as advised Hox $(2010), \mathrm{R}_{2}^{2}=\left(\sigma_{u(\mathrm{M} 2)}^{2}\right.$ $\left.\sigma_{u 0(\mathrm{M} 3)}^{2}\right) / \sigma^{2}{ }_{u(\mathrm{M} 2)}$, for which we considered $.01, .09$, and .25 a small, medium, and large effect, respectively (Cohen, 1988).

Assumptions. Some variables did not meet the required assumptions for multilevel analysis. First, we identified one univariate outlier for relevance perception (at intermediate test) with a standardized score $<-3.29$. We ran the analyses both without and with winsorizing this outlier (i.e., subtracting the difference between the two next lowest values of the next lowest value with standardized value $>-3.29$; Tabachnick \& Fidell, 2014), yielding similar results (results from the original data are reported). We did not identify any multivariate outliers using Mahalanobis' distance. Second, the assumption of univariate normality was violated for bias detection (moderate negative skewness) at the posttest and for bias explanation (moderate positive skewness) at the pretest and the intermediate test. When included in the multilevel models, we applied a square root transformation on both variables (Tabachnick \& Fidell, 2014). As bias detection was negatively skewed, the transformation was on the reflected variables (i.e., the subtracting each score from the largest score plus 1) that we re-reflected after transformation to enhance interpretation. After inspecting Q-Q plots (i.e., a probability plot of the standardized residuals against the values that would be expected under normality) for each multilevel model, we concluded that multivariate normality was met.

Absence and missing data. All 54 teachers filled out the pretest and the intermediate test and a final sample of 49 teachers completed the posttest. Within the control condition, three teachers dropped out after the intermediate test (although one did fill out the posttest teaching-attitudes questionnaire). Within the training condition, all 32 teachers attended the first session and completed the pretest and intermediate test, and 30 teachers completed the posttest. Eight teachers were unable to fully attend (one of) the next sessions (one could not attend session 2; six could not fully attend session 3; and one could not fully attend both sessions). Five of the seven teachers who did not (fully) attend session 3 were willing to complete the posttest online at home. We ran analyses both with and without the data of those (partly) absent teachers to assess how absence affected the results (dissimilar results are reported).

\footnotetext{
${ }^{2}$ Initially, we intended to run a model with Occasion as a random predictor before adding the cross-level interaction, but we lacked power to test for random slope variance, which is a common issue with categorical predictors. As recommended by LaHuis and Ferguson (2009), we checked further for cross-level interaction effects because fixed effects are estimated with more precision.
} 


\subsection{RESULTS}

To check the comparability of the experimental conditions, we first tested for significant differences on the background variables (Table 3.1) and on the outcome variables at the pretest. The training condition consisted of significantly more females and teachers from the economical teaching domain than the control condition (all teachers in training Group A were females from the same department). There were no other significant differences and the conditions did not differ on the pretest outcome variables, $t \mathrm{~s} \leq 1.63, p s \geq .109$. Table 3.2 displays the pretest, intermediate test, and posttest data per condition (Tables S3.1 and S3.2 in section 3.3. of the Supplementary Materials additionally display performance on the heuristics-and-biases and the vignettes for each task category separately).

Table 3.1. Comparison of participant characteristics between conditions

\begin{tabular}{lcccc}
\hline & Control & Training & & \\
\hline Chi-square tests to compare distribution & $\%$ & $\%$ & $\boldsymbol{X}^{\mathbf{2}(\mathbf{1})}$ & $\boldsymbol{p}$ \\
Gender - female & 46 & 75 & 4.88 & .027 \\
Teaching domain $^{\mathrm{a}}$ & - & - & 15.32 & $<.001$ \\
Experience critical thinkingb $^{\mathrm{b}}$ & - & - & 1.51 & .220 \\
Experience teaching statistics - yes & 14 & 19 & 0.25 & .620 \\
Experience teaching logic - yes & 9 & 13 & 0.15 & .695 \\
Experience teaching programming - yes & 14 & 9 & 0.24 & .624 \\
Experience student product assessment - yes & 91 & 100 & 3.02 & .082 \\
Independent t-tests to compare means & $\mathbf{M}$ (SD) & $\mathbf{M}(\mathbf{S D})$ & $\mathbf{t ( 5 2 )}$ & $\boldsymbol{p}$ \\
Age (years) & $46.4(10.6)$ & $44.0(8.5)$ & 0.9 & .373 \\
Teaching experience (years) & $7.0(6.8)$ & $9.5(6.5)$ & -1.4 & .182 \\
Need for Cognition (range: 1-6) & $4.5(0.5)$ & $4.5(0.5)$ & -0.4 & .705 \\
Actively Open-minded Thinking (range: $1-6)$ & $4.7(0.3)$ & $4.8(0.3)$ & -0.6 & .580 \\
\hline
\end{tabular}

Note. $n=22$ and $n=32$ for the control and training condition, respectively. a Domains were Technology $(n=10)$, Economics $(n=29)$, and Society $(n=15)$ with 2 degrees of freedom in Chi-square test instead of 1 . b Consisted of 6 ordinal answer categories, therefore we used a chi square test for trend for comparison. 
Table 3.2. Means (M) and standard deviations (SD) of the outcome variables at pretest, intermediate test, and posttest per condition

\begin{tabular}{|c|c|c|c|c|}
\hline & \multicolumn{2}{|c|}{$\begin{array}{l}\text { Control } \\
n=22^{\mathrm{a}}\end{array}$} & \multicolumn{2}{|c|}{$\begin{array}{c}\text { Training } \\
n=32^{\mathrm{b}}\end{array}$} \\
\hline & $M$ & SD & $\mathrm{M}$ & SD \\
\hline \multicolumn{5}{|c|}{ Learning tasks (range: $0-100 \%$ ) } \\
\hline Pretest & 53.7 & 21.1 & 61.5 & 14.4 \\
\hline Intermediate test & 57.9 & 16.8 & 74.7 & 13.3 \\
\hline Posttest & 52.9 & 20.1 & 73.1 & 17.8 \\
\hline \multicolumn{5}{|c|}{ Transfer tasks (range: $0-100 \%$ ) } \\
\hline Pretest & 48.9 & 13.2 & 42.7 & 21.8 \\
\hline Intermediate test & 54.2 & 16.4 & 51.0 & 21.1 \\
\hline Posttest & 53.5 & 18.9 & 43.3 & 29.1 \\
\hline \multicolumn{5}{|c|}{ Mental effort learning tasks (range: $1-9$ ) } \\
\hline Pretest & 3.9 & 0.9 & 4.2 & 1.2 \\
\hline Intermediate test & 4.2 & 1.0 & 4.8 & 1.2 \\
\hline Posttest & 3.9 & 1.0 & 4.3 & 1.2 \\
\hline \multicolumn{5}{|c|}{ Mental effort transfer tasks (range: $1-9$ ) } \\
\hline Pretest & 5.3 & 1.0 & 5.0 & 1.6 \\
\hline Intermediate test & 5.0 & 1.6 & 5.5 & 1.4 \\
\hline Posttest & 4.3 & 1.4 & 5.6 & 1.6 \\
\hline \multicolumn{5}{|c|}{ Bias detection (range: $0-5$ ) } \\
\hline Pretest & 2.6 & 1.5 & 2.8 & 1.1 \\
\hline Intermediate test & 1.6 & 1.3 & 2.4 & 1.2 \\
\hline Posttest & 2.8 & 1.5 & 4.6 & 0.5 \\
\hline \multicolumn{5}{|c|}{ Bias explanation (range: $0-5$ ) } \\
\hline Pretest & 1.3 & 1.5 & 1.1 & 1.0 \\
\hline Intermediate test & 0.3 & 0.6 & 0.7 & 0.9 \\
\hline Posttest & 0.6 & 0.7 & 1.8 & 1.3 \\
\hline \multicolumn{5}{|c|}{ Perceived relevance (range: 1-6) } \\
\hline Pretest & 5.0 & 0.5 & 5.2 & 0.5 \\
\hline Intermediate test & 5.0 & 0.7 & 5.1 & 0.5 \\
\hline Posttest & 4.8 & 0.8 & 5.1 & 0.6 \\
\hline \multicolumn{5}{|c|}{ Perceived competence (range: $1-6$ ) } \\
\hline Pretest & 4.3 & 0.7 & 4.0 & 0.8 \\
\hline Intermediate test & 4.2 & 0.6 & 3.6 & 0.9 \\
\hline Posttest & 4.1 & 0.7 & 4.0 & 0.6 \\
\hline
\end{tabular}

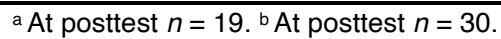




\subsubsection{HEURISTICS-AND-BIASES TASKS}

Average pretest performance on the learning and transfer tasks was, respectively, $58.3 \%$ (SD $=17.7)$ and $45.2 \%(\mathrm{SD}=18.9)$. Table 3.3 summarizes the results of the multilevel analyses that tested whether the training improved teachers' performance on learning (Hypothesis 1) and transfer.

Learning tasks. The ICC (M1) for learning-task performance revealed that $45 \%$ of the variance in performance on the instructed heuristics-and-biases tasks could be attributed to stable differences between teachers (level 2). Both adding Occasion as a predictor (M2) and, subsequently, adding a cross-level interaction between Occasion and Condition (M3) improved model fit significantly, M2: $\chi^{2}(2)=14.05, p<.001$; M3: $\chi^{2}(3)=21.65$, $p<.001$. The results revealed that, as compared to the control condition, teachers in the training condition did not improve significantly more on learning tasks from the pretest to the intermediate test, but did improve significantly more from pretest to posttest, Pre-Intermediate $\times$ Condition: $t(149)=1.82, p=.070$; Pre-Post $\times$ Condition: $t(149)=2.61, p=$ .010 . Thus, the training positively affected teachers' learning-task performance but this effect was apparent only after all three sessions (posttest). $\mathrm{R}_{2}{ }_{2}$ indicated that the predictors in the final model (M3) explained $28 \%$ of the variance between teachers' learning-task performance, which is a large overall effect. When excluding the teachers who were (partially) absent during the second or the third session, training effects were also statistically significant after the first session, Pre-Intermediate $\times$ Condition: $t(131)=2,13, p=.035$; Pre-Post $\times$ Condition: $t(131)=2.63, p=.010$.

Mental effort. As compared to the pretest, teachers in both conditions invested more mental effort in the learning tasks at the intermediate test $(p<.001)$ but not at the posttest (see Table S3.3, in section 3.3. of the Supplementary Materials).

Transfer tasks. The ICC for transfer-task performance indicated that $37 \%$ of the variance in performance on the not-instructed heuristics-and-biases tasks was due to stable differences between teachers. However, neither adding Occasion (M2) nor a cross-level interaction between Occasion and Condition (M3) could explain these differences, M2: $\chi^{2}(2)=5.04, p=$ $.080 ; \mathrm{M} 3: \chi^{2}(5)=8.11, p=.150$.

Mental effort. As compared to the pretest, teachers in the training condition invested more effort in the transfer tasks than teachers in the control condition at the intermediate test $(p=$ .018 ) and at the posttest ( $p<.001$; see Table S3.3, section 3.3. in Supplementary Materials). 
Table 3.3. Effect of critical thinking training on teachers' performance on learning and transfer (heuristics-and-biases) tasks

\begin{tabular}{|c|c|c|c|}
\hline & $\begin{array}{c}\text { M1: } \\
\text { intercept only }\end{array}$ & $\begin{array}{c}\mathrm{M} 2: \\
\text { effect occasion }\end{array}$ & $\begin{array}{c}\text { M3: } \\
\text { effect of training }\end{array}$ \\
\hline \multicolumn{4}{|l|}{ Learning tasks } \\
\hline Fixed part & Coefficient (s.e.) & Coefficient (s.e.) & Coefficient (s.e.) \\
\hline Intercept & $63.62(2.05)^{\star \star}$ & $58.33(2.50)^{\star \star}$ & $53.68(3.55)^{\star \star}$ \\
\hline Pre-Intermediate & & $9.52(2.50)^{\star *}$ & $4.22(3.78)$ \\
\hline Pre-Post & & $6.44(2.59)^{\star}$ & $-1.65(3.97)$ \\
\hline Condition & & & $7.85(4.61)$ \\
\hline Pre-Intermediate $\times$ Condition & & & $8.95(4.91)$ \\
\hline Pre-Post $\times$ Condition & & & $13.28(5.10)^{\star}$ \\
\hline \multicolumn{4}{|l|}{ Random part } \\
\hline Occasion and error variance $\left(\sigma_{e}^{2}\right)$ & $193.5(13.91)$ & $169.0(13.00)$ & $157.0(12.53)$ \\
\hline Teacher variance $\left(\sigma^{2} u o\right)$ & $160.1(12.65)$ & $167.8(12.96)$ & $120.3(10.97)$ \\
\hline Deviance & 1338.2 & 1324.1 & 1302.5 \\
\hline \multicolumn{4}{|l|}{ Transfer tasks } \\
\hline Fixed Part & Coefficient (s.e.) & Coefficient (s.e.) & Coefficient (s.e.) \\
\hline Intercept & $48.36(2.23)^{\star \star}$ & $45.22(2.88)^{\star \star}$ & $48.86(4.45)^{\star \star}$ \\
\hline Pre-Intermediate & & $7.10(3.19)^{\star}$ & $5.30(4.97)$ \\
\hline Pre-Post & & $2.23(3.29)$ & $4.75(5.22)$ \\
\hline Condition & & & $-6.16(5.78)$ \\
\hline Pre-Intermediate $\times$ Condition & & & $3.03(6.46)$ \\
\hline Pre-Post $\times$ Condition & & & $-4.02(6.70)$ \\
\hline \multicolumn{4}{|l|}{ Random part } \\
\hline Occasion and error variance $\left(\sigma_{e}^{2}\right)$ & $288.4(16.98)$ & $274.9(16.58)$ & $271.9(16.49)$ \\
\hline Teacher variance $\left(\sigma^{2} u 0\right)$ & $167.9(12.96)$ & $172.0(13.12)$ & $163.5(12.79)$ \\
\hline Deviance & 1388.2 & 1383.2 & 1380.1 \\
\hline
\end{tabular}

Note. Occasion is dummy-coded in two dummies (Intermediate test, Posttest) with Pretest as reference category. Condition coded $0=$ control, $1=$ training. ${ }^{\star} p<.05,{ }^{\star \star} p<.001$.

\subsubsection{DETECTING AND EXPLAINING REASONING BIASES}

At the pretest, teachers detected $2.7(\mathrm{SD}=1.3)$ out of five biases in a student product and correctly explained $1.2(\mathrm{SD}=1.3)$ biases. Both condition-averages decreased from pretest to intermediate test, whereas only the training condition increased from pretest to posttest on bias detection and explanation (Table 3.2). At all tests, the average number of correctly explained biases remained substantially behind the number of detected biases. Table 3.4 summarizes the analyses that tested whether the training significantly improved teachers' detection and explanation of reasoning biases (hypothesis $2 \mathrm{a}$ and $2 \mathrm{~b}$ ).

Bias detection. The ICC for bias detection revealed that only $8 \%$ of the variance in teachers' ability to detect biases was due to stable differences between teachers. Both Occasion (M2) and the Occasion $\times$ Condition interaction (M3) improved the model fit significantly, M2: $\chi^{2}(2)$ $=67.40, p<.001 ; \mathrm{M} 3: \chi^{2}(3)=30.61, p<.001$. As expected, the training positively affected 
teachers' ability to detect biases, but only after three sessions, Pre-Intermediate $\times$ Condition: $t(149)=1.45, p=.152$; Pre-Post $\times$ Condition: $t(149)=4.44, p<.001 . \mathrm{R}_{2}{ }_{2}$ indicated that the predictors in this final model explained $25 \%$ of the variability in teachers' ability to detect biases in student products, which is a large effect.

Bias explanation. $15 \%$ of the variance in teachers' ability to explain reasoning biases was due to stable differences between teachers (M1; ICC). Both adding Occasion and the Occasion $\times$ Condition interaction improved the model fit significantly, M2: $\chi^{2}(2)=21.65, p$ $<.001$; M3: $\chi^{2}(3)=17.93, p<.001$, showing that - as expected - the training positively affected teachers' ability to explain biases correctly, but (again) only after three sessions, Pre-Intermediate $\times$ Condition: $t(149)=1.20, p=.232$; Pre-Post $\times$ Condition: $t(149)=3.38, p<$ .001 . The predictors in this model explained $10 \%$ of the variability in bias explanation (a medium effect).

Table 3.4. Effect of critical thinking training on teachers' ability to detect and explain reasoning biases in a student product

\begin{tabular}{|c|c|c|c|}
\hline & $\begin{array}{c}\text { M1: } \\
\text { intercept only }\end{array}$ & $\begin{array}{c}\mathrm{M} 2: \\
\text { effect occasion }\end{array}$ & $\begin{array}{c}\text { M3: } \\
\text { effect of training }\end{array}$ \\
\hline \multicolumn{4}{|l|}{ Bias detection (sq. root) } \\
\hline Fixed part & Coefficient (s.e.) & Coefficient (s.e.) & Coefficient (s.e.) \\
\hline Intercept & $1.73(0.04)^{\star \star}$ & $1.67(0.05)^{\star \star}$ & $1.64(0.07)^{\star \star}$ \\
\hline Pre-Intermediate & & $-0.18(0.06)^{\star}$ & $-0.27(0.08)^{\star}$ \\
\hline Pre-Post & & $0.40(0.06)^{\star \star}$ & $0.09(0.09)$ \\
\hline Condition & & & $0.05(0.09)$ \\
\hline Pre-Intermediate $\times$ Condition & & & $0.16(0.11)$ \\
\hline Pre-Post $\times$ Condition & & & $0.50(0.11)^{\star \star}$ \\
\hline \multicolumn{4}{|l|}{ Random part } \\
\hline Occasion and error variance $\left(\sigma_{e}^{2}\right)$ & $0.17(0.4)$ & $0.09(0.30)$ & $0.08(0.27)$ \\
\hline Teacher variance $\left(\sigma^{2} u o\right)$ & $0.02(0.12)$ & $0.05(0.22)$ & $0.03(0.19)$ \\
\hline Deviance & 183.8 & 116.4 & 85.8 \\
\hline \multicolumn{4}{|l|}{ Bias explanation (sq. root) } \\
\hline Fixed Part & Coefficient (s.e.) & Coefficient (s.e.) & Coefficient (s.e.) \\
\hline Intercept & $0.74(0.06)^{\star \star}$ & $0.84(0.09)^{\star \star}$ & $0.83(0.13)^{\star \star}$ \\
\hline Pre-Intermediate & & $-0.39(0.11)^{\star \star}$ & $-0.53(0.16)^{*}$ \\
\hline Pre-Post & & $0.13(0.11)$ & $-0.31(0.17)$ \\
\hline Condition & & & $0.02(0.17)$ \\
\hline Pre-Intermediate $\times$ Condition & & & $0.25(0.21)$ \\
\hline Pre-Post $\times$ Condition & & & $0.72(0.21)^{\star \star}$ \\
\hline \multicolumn{4}{|l|}{ Random part } \\
\hline Occasion and error variance $\left(\sigma_{e}^{2}\right)$ & $0.39(0.62)$ & $0.31(0.56)$ & $0.28(0.53)$ \\
\hline Teacher variance $\left(\sigma^{2} u\right)$ & $0.07(0.26)$ & $0.10(0.31)$ & $0.09(0.30)$ \\
\hline Deviance & 319.6 & 298.0 & 280.1 \\
\hline
\end{tabular}

Note. Occasion is dummy-coded in two dummies (Intermediate test, Posttest) with Pretest as reference category. Condition coded $0=$ control, $1=$ training. ${ }^{*} p<.05,{ }^{* \star} p<.001$. 


\subsubsection{ATIITUDES TOWARDS TEACHING}

Average perceived relevance and perceived competence ratings on the teaching attitudes questionnaire were already quite high at pretest, at $5.1(\mathrm{SD}=0.5)$ and $4.1(\mathrm{SD}=0.8)$ on a six-point scale. Table 3.5 summarizes the results of the analyses that tested whether the training positively affected teachers' relevance perception (hypothesis $3 \mathrm{a}$ ) and competence perception (hypothesis 3b).

Table 3.5. Effect of critical thinking training on teachers' perceived relevance of and perceived competence in teaching critical thinking

\begin{tabular}{|c|c|c|c|}
\hline & $\begin{array}{c}\text { M1: } \\
\text { intercept only }\end{array}$ & $\begin{array}{c}\text { M2: } \\
\text { effect occasion }\end{array}$ & $\begin{array}{c}\text { M3: } \\
\text { effect of training }\end{array}$ \\
\hline \multicolumn{4}{|l|}{ Perceived relevance } \\
\hline Fixed part & Coefficient (s.e.) & Coefficient (s.e.) & Coefficient (s.e.) \\
\hline Intercept & $5.07(0.07)^{\star \star}$ & $5.11(0.08)^{\star \star}$ & $4.97(0.13)^{\star \star}$ \\
\hline Pre-Intermediate & & $-0.05(0.08)$ & $0.06(0.12)$ \\
\hline Pre-Post & & $-0.09(0.08)$ & $-0.12(0.13)$ \\
\hline Condition & & & $0.24(0.16)$ \\
\hline Pre-Intermediate $\times$ Condition & & & $-0.19(0.16)$ \\
\hline Pre-Post $\times$ Condition & & & $0.04(0.16)$ \\
\hline \multicolumn{4}{|l|}{ Random part } \\
\hline Occasion and error variance $\left(\sigma_{e}^{2}\right)$ & $0.17(0.41)$ & $0.17(0.41)$ & $0.17(0.41)$ \\
\hline Teacher variance $\left(\sigma^{2} u\right)$ & $0.20(0.44)$ & $0.20(0.44)$ & $0.19(0.43)$ \\
\hline Deviance & 249.0 & 247.8 & 243.7 \\
\hline \multicolumn{4}{|l|}{ Perceived competence } \\
\hline Fixed Part & Coefficient (s.e.) & Coefficient (s.e.) & Coefficient (s.e.) \\
\hline Intercept & $4.02(0.09)^{\star \star}$ & $4.12(0.10)^{\star \star}$ & $4.33(0.15)^{\star \star}$ \\
\hline Pre-Intermediate & & $-0.27(0.10)^{\star \star}$ & $-0.09(0.14)$ \\
\hline Pre-Post & & $-0.02(0.10)$ & $-0.15(0.15)$ \\
\hline Condition & & & $-0.35(0.20)$ \\
\hline Pre-Intermediate $\times$ Condition & & & $-0.30(0.19)$ \\
\hline Pre-Post $\times$ Condition & & & $0.22(0.19)$ \\
\hline \multicolumn{4}{|l|}{ Random part } \\
\hline Occasion and error variance $\left(\sigma_{e}^{2}\right)$ & $0.27(0.52)$ & $0.25(0.50)$ & $0.23(0.48)$ \\
\hline Teacher variance $\left(\sigma^{2} u\right)$ & $0.31(0.56)$ & $0.32(0.57)$ & $0.29(0.54)$ \\
\hline Deviance & 321.7 & 312.2 & 299.9 \\
\hline
\end{tabular}




\section{TRAINING CRITICAL THINKING AND TEACHING ATTITUDES}

Perceived relevance. Fifty-three percent of the variance in teachers' perceived relevance of teaching CT was due to stable differences between teachers (M1; ICC). Unexpectedly, neither adding Occasion (M2) nor the Occasion $\times$ Condition interaction (M3) further improved the model, M2: $\chi^{2}(2)=1.25, p=.535 ; \mathrm{M} 3: \chi^{2}(5)=5.33, p=.377$, indicating that the training did not affect teachers' perceived relevance of teaching CT.

Perceived competence. Fifty-three percent of the variance in teachers' perceived competence in teaching $\mathrm{CT}$ was due to stable individual differences (M1; ICC). Adding Occasion (M2) improved the model fit significantly, $\chi^{2}(2)=9.48, p=.009$. Although M3 showed an improved fit, $\chi^{2}(3)=12.33, p=.006$, neither of the added Occasion $\times$ Condition interactions in this model were individually statistically significant. The interactions pointed towards a temporary decrease in teachers' perceived competence from pretest to intermediate test and a slight increase from pretest tot posttest, Pre-Intermediate $\times$ Condition: $t(150)=-1.62$, $p=.107$; Pre-Post $\times$ Condition: $t(150)=1.13, p=.261$. The predictors explained $10 \%$ of the variability in teachers' perceived competence (a medium effect).

\subsection{DISCUSSION}

The aim of this study was to gain insight into how higher education teachers' skills and attitudes related to teaching $\mathrm{CT}$ could be fostered, which is a first step towards better preparing and supporting teachers for their crucial role in fostering students' CT-skill acquisition. We focused on an essential CT-skill: the ability to avoid bias in reasoning and decision-making, as measured with heuristics-and-biases tasks. The training focused on improving teachers' own performance on those tasks, their ability to detect and explain biases in student products, and their attitudes towards teaching CT.

\subsubsection{HEURISTICS-AND-BIASES TASKS}

Because there were no previous experimental studies available on the effects of CT-training for teachers, we first tested whether we would replicate a main finding in student populations, that explicit bias instruction combined with task practice improves performance on learning (i.e., instructed) tasks but not on transfer (i.e., not-instructed but related) tasks (e.g., Heijltjes et al., 2014). Indeed, we replicated this finding with teachers and found a large effect on teachers' performance on learning tasks (hypothesis 1). However, in contrast to prior single-session studies with students, our learning effect was only significant after three training sessions when considering the entire sample (note that the training condition did improve significantly at intermediate test when excluding absent teachers from the analyses). The explorative analyses indicated that the improved performance on learning tasks after three training sessions was attained with a similar amount of mental effort investment as prior to training. This is consistent with previous research with students (Heijltjes et al., 2014, 2015; Van Peppen et al., 2018) and points to an acquired efficiency when dealing with the tasks (Hoffman \& Schraw, 2010; Paas \& Van Merriënboer, 1993; Van Gog \& Paas, 2008). 
The lack of an effect on transfer task performance is in line with prior research with student populations (e.g., Heijltjes et al., 2014). Although we speculated that the generative learning activities (e.g., designing a CT-task) offered in the second and third session could positively affect transfer, we found no evidence that this was the case. We should note that the low reliability of the transfer tasks dramatically reduced the power to detect intervention effects (Kanyongo et al., 2007). In our study, this low reliability can at least partly be explained by, respectively, low variance due to floor and ceiling effects at all tests for the Wason selection tasks and the covariation detection tasks that we used to measure transfer (see Table S3.1 in section 3.3 of the Supplementary Materials). Nevertheless, the measurement of CT is still a major challenge (Ku, 2009; Liu et al., 2014). Not only did other studies using heuristics-and-biases tasks report low reliabilities (Aczel et al., 2015; Bruine de Bruin et al., 2007; West et al., 2008), but multiple studies also reported low reliabilities and/or poor construct validity on widely used standardized CT tests (e.g., California Critical Thinking Skills Test and Watson-Glaser Critical Thinking Appraisal; Bernard et al., 2008; Bondy et al., 2001; Jacobs, 1999; Leppa, 1997; Loo \& Thorpe, 1999). We do have two other indications that at least some transfer took place. First, the explorative analyses showed an increase in invested mental effort in the transfer tasks on the intermediate test and the posttest compared to the pretest, which may indicate that the trained teachers did detect a conflict between their heuristic response and the normative response and invested more effort in attempting to resolve this conflict, but (mostly) without success. Following this, Stanovich (2018) would explain the incorrect performance as an override failure due to insufficiently automatized mindware (i.e., requisite knowledge for correct reasoning), that is, subjects possess enough mindware to detect a conflict but not enough mindware to trigger the normatively correct response. More importantly, we found some evidence of transfer to other contexts, as the training improved teachers' ability to detect and explain instructed and non-instructed biases in student products (see also Table S3.2 in section 3.3. of the Supplementary Materials).

\subsubsection{DETECTING AND EXPLAINING REASONING BIASES}

Because improving teachers' own CT-skills is not sufficient for teaching it, we also examined whether the training positively affected other variables, specifically related to teaching CT. In line with our hypotheses $(2 \mathrm{a}$ and $2 \mathrm{~b}$ ), we found that the trained teachers detected significantly more biases in the student product (vignettes) and were better able to explain the biases correctly than teachers in the control condition. Yet again, these effects were only visible after three training sessions. The results of the intermediate test are hard to interpret, because performance in both the training and the control condition dropped substantially from pretest to intermediate test. This indicates a potential limitation of our study: despite their structurally equivalent features, this may indicate that the vignettes varied in difficulty level. Notably, despite the fact that the ability to explain biases significantly improved after three sessions, performance was still relatively low: the descriptive statistics revealed that while the trained teachers detected on average all biases hidden in the student products, 
they could explain only half of them correctly. From a teaching perspective this is a crucial shortcoming as providing adequate feedback on students' reasoning, is essential for students' CT-skills acquisition (Abrami et al., 2015).

\subsubsection{ATITUDES TOWARDS TEACHING}

In contrast to our hypothesis (3a) and to the findings of a training study in the domain of science teaching (Van Aalderen-Smeets \& Walma van der Molen, 2015), we found no effect of the training on teachers' perceived relevance of teaching CT. The most likely explanation is a ceiling effect, as all participating teachers already perceived the teaching of CT as highly relevant prior to the training. Because participation was voluntary, the participating teachers may have been positively biased regarding the relevance of teaching CT compared to the general teacher population.

Also in contrast to our hypothesis ( $3 \mathrm{~b}$ ) and Van Aalderen-Smeets and Walma van der Molen (2015), we found no positive effect of the training on perceived competence. Interestingly, perceived competence of teachers in the training session even seemed to drop temporarily from pretest to intermediate test. Possibly, the first training session made teachers more aware of their knowledge gaps and thereby temporarily decreased their perceived competence in teaching CT. In addition, it may also be possible that, in order to improve teachers' attitudes, the focus on attitudes should be stronger than was the case in our training. We intended to foster attitudes with the second and third training session through discussing the relevance of teaching CT and focusing on ways to integrate CT during teaching, but this may not have been sufficient and may require more time. In comparison, the training in the study by Van Aalderen-Smeets and Walma van der Molen (2015) was fully focused on improving teachers' attitudes towards teaching science and more intensive (i.e., six meetings of three hours, spread over six months).

\subsubsection{LIMITATIONS AND FUTURE RESEARCH}

Two potential limitations of this study arise from the fact that this study was conducted in the context of an authentic professional development course for teachers. First, we were not able to randomly assign teachers to the training and the control condition and, therefore, cannot draw strong causal conclusions on the effects of the training, although our pretest data did not give strong reasons to question the comparability of both conditions (i.e., no significant condition differences on the outcome measures and on most of the background variables). The training condition did consist of significantly more females and teachers from the economical teaching domain than the control condition (all teachers in training Group A were females from the same department), and we cannot fully rule out that this may have affected the outcome measures. However, neither gender nor teaching domain correlated with improvement on the outcome measures, indicating that there was no confound. 
Second, because participation in the training and the study was voluntary, our sample might not be representative for the overall population of higher education teachers. As mentioned earlier, this likely affected our findings regarding effects of training on teaching attitudes. Future research should attempt to address perceived relevance of and perceived competence in teaching $\mathrm{CT}$ in more representative teacher samples, because according to expectancy-value theory, these variables are important predictors of whether teachers will actually teach CT-skills in their classroom. Regarding the measures of the effects of training on teachers' CT-skills, this was presumably less problematic as we see no reason (given similar findings from prior research with students) to expect a different data pattern in a more varied sample. However, as our sample size was rather small and because this is the first experiment focusing on teachers' CT-skills, future research should point out whether our findings are replicated. Furthermore, what could possibly be a result of self-selection bias, is that the results of this study (and a previous survey study; Janssen et al., 2019) suggest that higher education teachers perform relatively well (compared to students) on the heuristics-and-biases tasks even prior to any intervention.

Another potential limitation is that we cannot draw conclusions on what specific aspects of the training were most effective for which outcomes. Because there were no experimental studies available on training CT-skills of (higher education) teachers, our first step was to explore whether CT-training would have the potential to affect teachers' CT-skills and attitudes towards teaching CT. Future research on teacher training should address this question and our findings suggest that such research should not only focus on what is most effective for establishing improvement in teachers' performance on CT-tasks, but particularly on how to improve their performance on tasks that more closely related to the teaching practice, like the detection and explanation measures we introduced in this study. These measures can be seen as direct indicators of an essential condition necessary for teaching CT, namely providing adequate feedback so that students know how to improve their reasoning. As explaining why particular arguments were invalid was especially hard for teachers, we suggest that (research on) teacher CT-training should particularly focus on how to further improve this ability.

Finally, because we found clear effects on our outcome measures only after three training sessions, and even found a temporary drop in teachers' competence perception after one session, it seems important to investigate issues concerning the amount of training time needed and spacing and repetition of practice opportunities. Spacing and repetition might also be effective means to foster deep learning and, thereby, increase transfer to other tasks and contexts, and increase the ability to explain students' reasoning biases, which are both very important in teaching CT. Especially given the constraints of time available for professional development in practice, insight into efficient training programs that nevertheless provide teachers with enough time to acquire the complex skill of CT and to gain confidence in their ability to teach it are needed.

In a future study, it would be desirable to replicate the findings using a full experimental design with a larger and more representative teacher sample. In addition, it would also be worthwhile to test specific interventions that focus on impacting one of the outcome measures addressed in our study (e.g., bias explanation or teaching attitudes). 


\subsubsection{CONCLUSION}

To the best of our knowledge this was the first study that explored the potential effects of CT-training on teachers' CT-skills and attitudes. Where previous research largely focused on how to improve students' CT-skills, we focused on teachers' CT-skills. In line with previous studies with students, our study provides evidence for the trainability of teachers' CT-skills, but it also shows that the skills and attitudes needed for teaching CT do not improve automatically. Our findings highlight the importance of supporting teachers in their challenging but crucial role of fostering students' CT-skills and ask for further research into the best ways to promote teachers' ability to transfer trained skills to other CT-tasks and their ability to explain students' reasoning and to foster their attitudes towards teaching CT. 
Chapter 4

Training higher education

teachers' ability to explain biases

in students' reasoning

An adapted version of this chapter is submitted for publication as:

Janssen, E. M., Mainhard, T., Verkoeijen, P. P. J. L., Heijltjes, A. E. G., Van Peppen, L. M., \& Van Gog, T. (submitted). Training Higher Education Teachers' Ability to Explain Biases in Students' Reasoning. Manuscript submitted for publication.

\section{Acknowledgement of author contributions:}

EJ, TM, PV, AH, and TVG designed the study, EJ and AH recruited participants and collected the data, EJ analyzed the data, TM checked the data package, EJ drafted the manuscript, all authors contributed to critical revision of the manuscript, TM, PV, AH, and TVG supervised the study. 


\section{ABSTRACT}

Higher education teachers face the complex task of improving students' critical thinking (CT). This study investigated whether teachers can be trained in explaining biases in students' reasoning. Prior research showed they struggled with this crucial skill for teaching CT (i.e., providing feedback). Teachers in the control condition only received CT-training on avoiding bias in reasoning. Teachers in the experimental condition additionally received training on how to explain biases in students' reasoning (in essays/papers). As hypothesized, teachers in the experimental condition showed pre-to-posttest improvements in explaining trained biases at immediate posttest, whereas the control condition did not. This improvement was maintained at delayed posttest two weeks later, but - surprisingly - the control condition had also improved. Improvement in explaining non-trained biases (i.e., transfer) was very small and did not differ between conditions. This study suggests that one important aspect of CT-teaching, namely the ability to explain biases in students' reasoning, is trainable.

Keywords. critical thinking; heuristics and biases; teaching and teacher education; instructional design; higher education. 


\section{CHAPTER 4}

\subsection{INTRODUCTION}

An important objective of higher education is to foster students' critical thinking (CT) skills. CT-skills, however, do not develop automatically as a 'by-product' of higher education. Two large-scale longitudinal studies in the United States showed that students' CT-skills hardly improved over the college years (Arum \& Roksa, 2011; Pascarella et al., 2011; although others are more optimistic Huber \& Kuncel, 2016). According to a meta-analysis, the most effective intervention for improving students' CT-skills is a combination of authentic instruction, dialogue, and mentoring (Abrami et al., 2015). Because teachers are responsible for providing such interventions, they face a challenging task. Surprisingly, even though review studies highlighted the crucial role of teachers (Abrami et al., 2008; Pithers \& Soden, 2000; Ritchhart \& Perkins, 2005), there is a paucity of research focusing on how to equip teachers for teaching CT (Janssen, Mainhard, et al., 2019; Lorencová et al., 2019; Paul et al., 1997).

The present study focuses on one important aspect of CT, namely, the ability to avoid bias in reasoning and decision-making (Baron, 2008; Facione, 1990a; Perkins et al., 2000; West et al., 2008), and investigates effects of training higher education teachers' ability to explain biases in students' reasoning, which is critical for teaching $\mathrm{CT}^{1}$. To assess biases in reasoning, researchers have designed heuristics-and-biases tasks. These are reasoning problems in which an intuitively cued heuristic response conflicts with normative rules of rationality as set for instance by logic or probability (Tversky \& Kahneman, 1974), consider the following example:

Premise 1: If ports follow safety guidelines, they are protected sufficiently against safety risks

Premise 2: The port of Rotterdam does not follow safety guidelines,

Conclusion: The port of Rotterdam is not protected sufficiently against safety risks

Does the conclusion follow necessarily from the premises?

Because of its believability, most people intuitively conclude that it does (cf. the belief-bias effect; Markovits \& Nantel, 1989; Sá, West, \& Stanovich, 1999). However, the correct answer requires replacing the intuitive, or heuristic response ("yes, of course a port is not sufficiently protected without following any safety guidelines") with a logical response ("the first premise does not imply anything about the consequences of not following guidelines"). Thus, if a safety-management student concludes in an assignment that the port of Rotterdam

${ }^{1}$ In this article, the term "higher-education teachers" refers to post-secondary teachers employed at a university. 
is not protected against safety risks, merely based on the observation that the port was not following particular guidelines (without recognizing that the port may have used a different way to protect itself), the teacher should be able to provide a clear explanation of why that student's argument is incorrect ${ }^{2}$.

While research has shown that explicit instruction combined with opportunities for practice improves students' performance on heuristics-and-biases tasks (Heijltjes et al., 2014, 2015; Van Peppen et al., 2018), very little research is available on how teachers can best be trained to provide such instruction and feedback to their students (Janssen, Mainhard, et al., 2019; Janssen, Meulendijks, et al., 2019). The (limited) research with teachers available thus far, suggests teachers' own ability to avoid biased reasoning could be trained, but did not necessarily make teachers better equipped for teaching CT. That is, a quasi-experimental training study showed that providing teachers with instructions on reasoning biases and how to avoid them, followed by practice tasks, had a large effect on their ability to avoid those biases (evidenced by their performance on test tasks) and their ability to detect these biases in students' written arguments, compared to a no-training control group (Janssen, Mainhard, et al., 2019). However, teachers hardly improved in correctly explaining why a students' argument was incorrect, which is crucial for providing adequate feedback to students during instruction, dialogue and mentoring sessions (which, in turn, are essential for students' CT-skills acquisition; Abrami et al., 2015).

In sum, training teachers' own CT-skills is necessary but not sufficient to equip them for teaching CT. Training should additionally focus on skills more directly related to teaching CT. Therefore, the present study investigated the effects of providing teachers with additional explanation training on their ability to explain students' reasoning biases and - also - on their attitudes towards teaching CT. For teachers to engage in teaching CT in their daily practice, they not only need to possess CT-skills and CT-teaching-skills, they also need to have positive attitudes towards teaching CT. In line with expectancy-value theory (Eccles \& Wigfield, 2002), teachers with a positive attitude towards the relevance of teaching a particular subject (i.e., high task value) and confidence in one's ability (i.e., high expectancy of success) were found to be more likely to engage in effective teaching (Choy \& Cheah, 2009, 2009; Klassen \& Tze, 2014; Paul et al., 1997; Van Aalderen-Smeets \& Walma van der Molen, 2013, 2015). In two previous studies, most teachers strongly agreed on the relevance of teaching CT and moderately agreed on statements addressing confidence in their own ability to teach CT (Janssen, Mainhard, et al., 2019; Janssen, Meulendijks, et al., 2019). Training did not further affect perceived relevance of teaching CT (possibly because it was already high prior to training; Janssen, Meulendijks, et al., 2019). Surprisingly, better CT-task performance was not (directly) related to higher perceived competence in teaching CT (Janssen, Mainhard, et al., 2019; Janssen, Meulendijks, et al., 2019). In fact, in a training study, performance increased

\footnotetext{
${ }^{2}$ In research on reasoning, it is debated whether heuristic responses should be labelled "incorrect" or "biased" (for a review, see Stanovich \& West, 2000). For the sake of simplicity we use the terms "correct" response or "logical" response for the responses that are considered normatively correct following the rules of logic and "incorrect" for responses that are not normatively correct according to the rules of logic
} 
yet perceived competence decreased after the first training session (Janssen, Mainhard, et al., 2019). Although this drop was temporary (perceived competence increased again after the last training session), this counterintuitive finding highlights the importance of measuring the effects of training not only on teachers' skills, but also on their attitudes.

\subsubsection{THE PRESENT STUDY}

The present study experimentally investigated whether extending CT-training on avoiding biases (cf. Janssen, Mainhard, et al., 2019) with an individual online training on how to explain reasoning biases in students' written arguments, would improve teachers' ability to explain reasoning biases and affect their perceived relevance of and perceived competence in teaching CT. The control condition participated in the CT-training on avoiding biases only. The experimental condition additionally followed an explanation training. The explanation training focused on: how to (1) detect, (2) explain, and (3) improve biased reasoning in students' writing. The training was presented in an online learning environment and relied on example-based learning (Van Gog et al., 2019). Teachers first received a fully worked-out example of all three steps; then a partially completed example in which they completed step 1 themselves; and, finally, had to complete the three steps without guidance (cf. the 'fading' principle; Renkl \& Atkinson, 2003). Prior to the training, immediately afterwards, and two weeks later, teachers completed a CT-test assessing their ability to explain the reasoning biases instructed during the CT-training. Teachers in de experimental condition had practiced with explaining half of the reasoning biases that the test addressed (increased performance on non-practiced biases would be indicative of transfer). In addition, teachers reported their invested mental effort (Paas, 1992) in providing the explanations, and their attitudes towards teaching CT (Janssen, Mainhard, et al., 2019).

Hypotheses. We hypothesized that teachers in the experimental condition would improve more than teachers in the control condition in their ability to explain the practiced biases as evidenced by increased performance from the pretest to the immediate posttest, and expected this effect to remain over time (i.e., still present at the delayed posttest).

Explorative analyses. We explored whether the explanation training also had a positive effect on transfer, that is, whether teachers' explanations of reasoning biases they had not practiced, but had similar underlying principles as biases they did practice, also improved. Findings on transfer have been mixed so far (Heijltjes et al., 2014, 2015; Van Peppen et al., 2018), but example-based learning and the fading principle have been shown to enhance transfer of other cognitive skills (Van Gog et al., 2019). We also explored whether the explanation training affected teachers' (self-reported) mental effort investment in explaining biases (Paas, 1992). Combined with performance data, this gives more detailed insight into the efficiency of the training (Paas \& Van Merriënboer, 1993; Van Gog \& Paas, 2008; see also previous training studies on avoiding bias, Heijltjes et al., 2014; Janssen, Mainhard et al., 2019; Van Peppen et al., 2018). Finally, we explored whether the explanation training (with its explicit focus on a crucial aspect of teaching CT) positively affected teachers' perceived relevance of and perceived competence in teaching CT. 


\subsection{METHOD}

\subsubsection{PARTICIPANTS AND DESIGN}

Participants were 40 teachers from the study program Safety and Security Management at a Dutch university of applied sciences ${ }^{3}$. Three participants had to be excluded from the analyses (one was not a native Dutch speaker, and two teachers completed the pretest only), resulting in a sample of 37 (Age: $\mathrm{M}=46.8$ years, $\mathrm{SD}=9.9$; Teaching Experience: $\mathrm{M}=8.0$ years, SD = 5.2; Sex: 16 females, 21 males; Level of Education: 14 had an applied-university degree, 22 a research-university degree, and 1 had attended a research university yet never graduated). The teachers were randomly assigned to the control condition (CT-training only; $n=19$ ) or the experimental condition (CT-training plus online explanation training; $n=18$ ). Participating in the training was a mandatory part of professional development (imposed by the faculty management); participating in the study (i.e., giving informed consent to the use of their data on part of the training for research) was, however, voluntary.

The study design was pre-approved by the faculty ethics review board of the first author's institute. Hypotheses and statistical analyses were preregistered via the As Predicted format. The preregistration and the study data are stored on an Open Science Framework (OSF) page for this project, see https://osf.io/8h4me/?view_only=a1101a3fd9c9413da5flad436b994ea6 (anonymized view-only link for the purpose of masked review).

\subsubsection{MATERIALS}

An overview of the study design is shown in Figure 4.1.

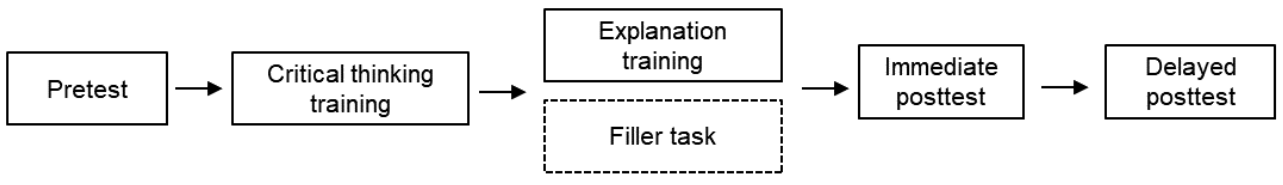

Figure 4.1. Overview of the study design.

\section{Training materials.}

Critical thinking training. As noted, all teachers participated in the same interactive CT-training focused on avoiding reasoning biases (duration approximately 150 minutes). The training was an adapted version of the first training session in our previous study (Janssen, Mainhard,

\footnotetext{
${ }^{3}$ The Dutch education system distinguishes between higher education at an academic university (i.e., Bachelor or Master at a research university) or non-academic university (i.e., Bachelor or Master at a university of applied sciences).
} 
et al., 2019) and was given by the first and fourth author in four separate groups $(n=8, n=$ $7, n=14$, and $n=8$ ). The goal was to introduce the concept biased reasoning and to practice with avoiding bias in teachers' own thinking and reasoning. Teachers were trained in avoiding belief bias in syllogistic reasoning (i.e., tendency to be influenced by the believability of a conclusion when evaluating the logical validity of arguments, see e.g.,Markovits \& Nantel, 1989; Newstead et al., 1992) and in avoiding confirmation bias in Wason selection tasks (tendency to verify rules/hypotheses rather than to falsify them, see e.g.,Dawson et al., 2002; Nickerson, 1998). The content of all training and test materials was adapted to the teachers' educational program Safety and Security Management. Extra information is provided in the Supplementary Materials (section 4.1).

Explanation training. After the CT-training, teachers in the experimental condition followed an individual, online training unit in which they practiced with explaining the just instructed biases in students' syllogistic reasoning. The explanation training relied on example-based learning (Van Gog et al., 2019) and applied the fading principle (Renkl \& Atkinson, 2003). We first showed teachers a text written by students containing a belief-biased conclusion, which was logically invalid (see Figure 4.2), along with a worked example of the three steps on (1) how to detect this type of bias; (2) how to explain the bias using the laws of logic; and (3) how to provide a concrete suggestion to improve the logic of the students' argument. Hereafter, teachers received a second text containing a similar bias along with a completion assignment in which teachers completed step 1 themselves, received accuracy feedback, and then saw a worked example of step 2 and 3. Finally, teachers received a third text with a similar bias and had to complete all steps themselves. There were two sets of 'fading examples', in the first set the belief-biased conclusions concerned logical fallacies in the form of 'denial of the antecedent' arguments (if $p$ then $q$, not $p$ therefore not q; invalid, cf. Evans, 2002) and in the second set it concerned logical fallacies in the form of 'affirmation of the consequent' arguments (if p then q, q therefore p; invalid, cf. Evans, 2002). Teachers could practice at their own pace with these six tasks, for a maximum of 45 minutes.

\section{Students wrote the following in a group assignment where they evaluated the safety policy of the Port of Rotterdam:}

The aim of the SOLAS (Safety of Life at Sea) guidelines is to minimize the harm caused by incidents as much as possible. If the SOLAS guidelines are followed, the risk of incidents with harmful consequences is minimized (IMO, 2017). Because a recent inspection investigation showed that the Port of Rotterdam does not follow all of the SOLAS guidelines, we know that the port does not sufficiently minimize the risk of incidents with harmful consequences. In Chapter 3 we make some recommendations to improve the safety policy of the port.

Explain students why their argument is invalid.

Explain students how they could improve their argument.

Figure 4.2. Task example of a students' text containing a belief-biased conclusion (cf. denial of the antecedent). 
Filler task. Teachers in the control condition completed an individual, online filler task after the CT-training. The filler task was not related to explaining biases - yet relevant in the professional development context (i.e., dealing with sensitive topics in the classroom, such as conspiracy theories). Teachers worked at their own pace for maximally 45 minutes.

Tests. All tests were administered as an online survey with a forced-response format using Qualtrics Survey Software (Qualtrics, Provo, UT; http://www.qualtrics.com).

Background variables. Next to Sex, Age, Teaching Experience, and Level of Education, we asked five questions addressing teachers' Previous Experience potentially relevant for the skill to explain logical reasoning biases. First, teachers answered the question "For how many hours (estimation) have you been actively involved with the theme 'reasoning biases' in the past two years (think for example of following a workshop, teaching lessons, or developing lesson materials)?" Answering categories were: 0 hours, 1-20 hours, 21-40 hours, 41-60 hours, 61-80 hours, 81-100 hours, or $>100$ hours. Second, teachers answered four yes/no questions that asked whether they had followed a course on logic, taught such a course, followed a programming course, or taught a programming course.

Bias explanation tasks. To measure teachers' ability to explain biases in students' work, we constructed 21 vignettes (i.e., seven for each Test Occasion), based on actual student papers from the teachers' study program (Safety and Security Management). Each vignette (about 100 words) was presented as an excerpt from a student paper (written in the context of a group assignment) and in each vignette a logically invalid (yet seemingly plausible) conclusion was drawn (see Figure 4.2). Teachers were instructed to read the texts and to provide an explanation to students why the conclusion was incorrect and to provide a suggestion on how to improve the logic of the argument. The full test instruction and an example explanation to the students in Figure 4.2 are provided in Section 4.2 and 4.3 of the Supplementary Materials, respectively.

During each Test Occasion, four out of seven vignettes contained a belief-biased conclusion in students' syllogistic reasoning (twice denial of the antecedent; twice affirmation of the consequent) and three contained a confirmation-biased conclusion in students' reasoning about the verification of rules/hypotheses, as manifested in Wason selection tasks (task examples are provided in Section 4.4 of the Supplementary Materials). As the experimental condition practiced with explaining bias in the syllogistic-reasoning tasks, those four tasks allowed for examining whether the explanation training affected teachers' learning (from now on referred to as 'Learning Tasks'). Teachers did not practice with explaining the Wason selection tasks (though these had been part of the CT-training all teachers received), so those three tasks allowed for examining transfer (from now on referred to as 'Transfer Tasks').

The seven tasks were presented in randomized order. Each Test Occasion contained structurally equivalent student texts (i.e., same type of bias and fallacy), but with different surface features (i.e., different cover stories). All tasks were pilot-tested by five teachers from the same university of applied sciences (who were not participants in this study) to check whether the instruction was clear, to get an idea of the difficulty level of the tasks (to prevent ceiling or floor effects), and to assess time on task. Based on this pilot, we made some small textual changes. 
Coding scheme. Previous studies that coded participants' explanations of syllogistic reasoning problems (e.g., Hawkins et al., 1984; Markovits et al., 1996; Venet \& Markovits, 2001) distinguished between different types of correct statements about logic (e.g., simply repeating the major premise or referring to the possibility of another antecedent leading to the same outcome) and statements not involving logic (e.g., referring to real-world knowledge or to authority). Based on these schemes (in particular Markovits et al., 1996) we developed a coding scheme for the teacher explanations in the current study. Points were assigned for correctly explaining why the student's conclusion was logically invalid (0-3 points) and for correctly explaining how the student's argument could be improved ( $0-2$ points), resulting in a total score of 5 points per task. Table 4.1 provides an overview of all code definitions along with coded examples (Table S4.1 and S4.2 in Supplementary Material provide more coded examples). We also kept track of how often teachers used invalid logic in their explanations to the students (coded example: "It is better to conclude that if the port of Rotterdam does not follow all SOLAS guidelines, the damage caused by possible incidents will not be sufficiently limited." [invalid; not $\mathrm{p}$ therefore not q]). If an explanation provided both a logically correct statement on the student's argument and also a logically incorrect statement, we did not deduct points that were assigned to the correct statement (coded example: "SOLAS can make a contribution, but that does not mean that its absence does not lead to higher risks [invalid; not $\mathrm{p}$ therefore not, not, not, q]. Other guidelines may also ensure that the risk is minimized [valid: Counter-example explanation]").

Two coders scored about $25 \%$ of the data (all data of 10 randomly selected teachers). Interrater reliability was assessed on the variables as included in the statistical analyses by calculating a two-way mixed, consistency, single-measures intra-class correlation (ICC; McGraw \& Wong, 1996). The resulting ICCs were in the good and excellent range (Cicchetti, 1994). That is, the ICC per Test Occasion (pretest, immediate posttest, and delayed posttest) was $.81, .71$, and .86 for Learning Tasks and $.67, .91$, and .84 for Transfer Tasks ${ }^{4}$. The remainder of the data was scored by one coder. Cronbach's alpha per Test Occasion was .20, .80 , and .52 for Learning Tasks and $.14, .72$ and .75, for Transfer Tasks. Low reliability of the pretest scores is likely due to lack of knowledge (guessing); we return to this issue in the discussion.

Perceived mental effort. After each task, participants rated how much Mental Effort they invested on a 9-point rating scale ranging from (1) very, very low effort to (9) very, very high effort (Paas, 1992).

Attitudes towards teaching critical thinking. Attitudes towards teaching CT were assessed as Perceived Relevance of Perceived Competence in teaching CT (3 items per construct; Janssen, Mainhard et al., 2019). An example item of Perceived Relevance is "Learning outcomes will improve from critical thinking during educational activities." An example item of Perceived Competence is "I can explain clearly to my students how they are drawing incorrect conclusions from the available information." (rated on a six-point scale ranging from (1) strongly disagree to (6) strongly agree). Averaged scale scores could therefore range from 1 to 6 . Cronbach's alpha per Test Occasion was respectively $.50, .67$, and .77 for

\footnotetext{
${ }^{4}$ Because we also report the code frequencies in the results, we additionally calculated ICCs (two-way mixed, absolute agreement, single-measures) for each code category separately. The resulting ICCs ranged from fair to excellent and are reported in Table S4.3 in the Supplementary Material.
} 
Perceived Relevance and 42, .74, and .74 for Perceived Competence. Hence, whereas the pretest scores showed low reliability it was acceptable at the posttests (we return to this issue in the discussion).

Table 4.1. Overview of coding scheme with coded examples (translated from Dutch) belonging to the task shown in figure 4.2 (cf. denial of the antecedent)

\begin{tabular}{|c|c|c|}
\hline \multicolumn{3}{|c|}{ Explain students why their argument is invalid } \\
\hline Code & Definition & Coded example \\
\hline $\begin{array}{l}\text { no explanation } \\
\text { (Op) }\end{array}$ & $\begin{array}{l}\text { The explanation does not contain any } \\
\text { (correct) statements about logic of } \\
\text { the students' argument }\end{array}$ & $\begin{array}{l}\text { "Goal of SOLAS is to minimize harm } \\
\text { caused by incidents. That is not the } \\
\text { same as minimizing the risk of } \\
\text { incidents with harmful consequences." }\end{array}$ \\
\hline detection (1p) & $\begin{array}{l}\text { The explanation shows recognition of } \\
\text { the invalid conclusion }\end{array}$ & $\begin{array}{l}\text { "You cannot conclude that if SOLAS is } \\
\text { not fully followed, there will be } \\
\text { insufficient minimization of incidents." }\end{array}$ \\
\hline $\begin{array}{l}\text { information-lack } \\
\text { explanation }(2 p)\end{array}$ & $\begin{array}{l}\text { The explanation points out what } \\
\text { necessary information is lacking to } \\
\text { draw the students' conclusion }\end{array}$ & $\begin{array}{l}\text { "... As a reader, I don't know what the } \\
\text { reason is that not all guidelines are } \\
\text { followed by the port of Rotterdam." }\end{array}$ \\
\hline $\begin{array}{l}\text { counter-example } \\
\text { explanation }(3 p)\end{array}$ & $\begin{array}{l}\text { The explanation demonstrates } \\
\text { through a counter example how the } \\
\text { students' argument can also yield a } \\
\text { different conclusion }\end{array}$ & $\begin{array}{l}\text { “... The port of Rotterdam may also } \\
\text { follow other policy / (non-SOLAS) } \\
\text { guidelines, so that harmful } \\
\text { consequences are still reduced." }\end{array}$ \\
\hline \multicolumn{3}{|c|}{ Explain students how they could improve their argument } \\
\hline Code & Definition & Coded example \\
\hline $\begin{array}{l}\text { no suggestion } \\
(0 p)\end{array}$ & $\begin{array}{l}\text { The explanation does not contain } \\
\text { concrete (correct) suggestions to } \\
\text { improve the logic of the students' } \\
\text { argument }\end{array}$ & $\begin{array}{l}\text { "Through a more thorough } \\
\text { investigation." }\end{array}$ \\
\hline $\begin{array}{l}\text { reservation } \\
\text { suggestion (1p) }\end{array}$ & $\begin{array}{l}\text { The explanation suggests to place a } \\
\text { reservation to the conclusion }\end{array}$ & $\begin{array}{l}\text { "... it may be possible that the port } \\
\text { does not sufficiently minimize the risk } \\
\text { of incidents with harmful } \\
\text { consequences." }\end{array}$ \\
\hline $\begin{array}{l}\text { required- } \\
\text { information } \\
\text { suggestion }(2 p)\end{array}$ & $\begin{array}{l}\text { The explanation provides a } \\
\text { suggestion on concrete information } \\
\text { that would allow the students to draw } \\
\text { their original conclusion }\end{array}$ & $\begin{array}{l}\text { "... Indicate that they do not follow } \\
\text { SOLAS guidelines and that you will } \\
\text { check what they actually do to minimize } \\
\text { the risk." }\end{array}$ \\
\hline
\end{tabular}

Note. For conciseness we did not copy the full explanations to the 'Coded example' column, only the phrases that were illustrative of the code definition. 


\subsubsection{PROCEDURE}

Two weeks before the start of the training, teachers received a request via email to complete the pretest. The pretest first addressed background variables. Hereafter, teachers completed the questionnaire on attitudes towards teaching CT, and the bias explanation tasks along with Mental Effort ratings, respectively.

At least one day before attending the training session, all teachers had completed the pretest at home ${ }^{5}$. During the training session of 240 minutes in total (including breaks), teachers engaged in the CT-training (ca. $150 \mathrm{~min}$ ), the explanation training or filler task (45 $\mathrm{min})$, and the immediate posttest $(45 \mathrm{~min})$. After the CT-training, teachers worked individually on their laptop and received an e-mail with a hyperlink that randomly directed them to either the online explanation training (experimental condition) or to the online filler task (control condition). Once they finished (or after $45 \mathrm{~min}$. passed), teachers were directed to the immediate posttest.

Two weeks later, the teachers returned for a second professional development session, of which the first 45 minutes were reserved for the delayed posttest ${ }^{6}$. Because we wanted to test whether teachers could apply the laws of logic in their feedback to students and not whether they remembered the rules correctly, the four laws of logic that had been instructed in the first training session, were displayed on the board in abstract form (if $p$ then $q$ etc.) during both posttests ${ }^{7}$. After the delayed posttest, we debriefed teachers on the study purpose and provided hyperlinks to both the explanation training and the filler task (so that teachers had access to all learning materials).

\subsubsection{DATA ANALYSIS}

To examine how the explanation training affected teachers' bias explanations (Learning Tasks, Transfer Tasks, Mental Effort) and attitudes towards teaching CT (Perceived Relevance, Perceived Competence), we employed multilevel analyses for each outcome measure. We used the 'Ime4' package in R (Bates et al., 2015; R Development Core Team, 2008) and employed likelihood ratios to evaluate the fit between a multilevel model and the data. For every outcome measure, we started with an intercept-only model (M1) that decomposed the variance of the outcome measure into random error and differences between measurement

\footnotetext{
${ }^{5}$ Upon arrival of the teachers of the first training group, it appeared that one teacher had not received the pre-test in his e-mail (due to a miscommunication about the group he was in). He agreed, however, to take the test immediately and, consequently, joined the training session 20 minutes later.

${ }^{6}$ Six teachers were unable to attend the second training session within their own training group and therefore attended the second training session of one of the other training groups (i.e., consequently, three teachers completed a delayed posttest one week after the training and three completed it three weeks after the training).

${ }^{7}$ Due to practical circumstances, the laws of logic were not shown on the board at immediate posttest for one group.
} 
occasions (level $1 ; \sigma_{e}^{2}$ ) and variance attributed to stable differences between teachers (level 2; $\sigma_{u 0}^{2}$ ). In the second model (M2) we entered Test Occasion as fixed predictor (dummy-coded with the pretest as the reference category). Third (M3), we added a cross-level interaction between Test Occasion and Condition (with the control condition as reference category), which tested whether teachers in the experimental condition progressed differently on the outcome measures than teachers in the control condition. We used M2 as baseline model to compute the effect size of the experimental manipulation $\left(\mathrm{R}_{2}^{2}\right)$. As advised by Hox (2010), we calculated how much additional variance between teachers' scores was explained by the added Condition predictor in the final model, $\mathrm{R}_{2}^{2}=\left(\sigma_{u \theta(\mathrm{M} 2)}^{2}-\sigma^{2}{ }_{u(\mathrm{M} 3)}\right) / \sigma^{2}{ }_{u(\mathrm{M} 2)}$, for which we considered .01, .09, and .25 a small, medium, and large effect, respectively (Cohen, 1988).

Because there were no prior studies on which we could base a plausible prespecification of the model's parameters (e.g., standard errors or effect sizes), it was not possible to perform an a-priori multilevel power calculation. Therefore, we conducted a sensitivity analysis in $\mathrm{G}^{*}$ Power (Faul et al., 2007) assuming an ANOVA with a Test-Occasion $\times$ Condition effect, under a fixed alpha level of .05 , a power of .80 , and a correlation of .50 among repeated measures. Results indicated that a sample of $n=40$ would be sufficient to pick up small to medium effects $(f=0.20)$, and $n=30$ to pick up medium effects $(f=0.24)$ when considering $0.10,0.25$, and .40 as the cut-offs for small, medium, and large effects, respectively (Cohen, 1988). Given that our multilevel analyses were equivalent in power to ANOVA (yet robust to sphericity violations) our sample size should be sufficient to detect medium to large effects of the explanation training (Misangyi et al., 2006).

We tested for multivariate normality and multivariate outliers for each multilevel model by inspecting Q-Q plots and conducting Mahalanobis distance analyses. Assumptions were met, except for Transfer Tasks: data was not normal (positively skewed) and one multivariate outlier was identified. We therefore log-transformed the scores on Transfer Tasks (and report the untransformed means for ease of interpretation).

\subsection{RESULTS}

To check the comparability of the two experimental conditions, we first tested for significant differences on background variables and pretest scores. As Table 4.2 indicates, the control condition contained significantly more males and teachers who had previously taken a logic course than the experimental condition. In addition, the control condition had a significantly higher pretest score on Transfer Tasks, Welch's $t(27.9)=2.28, p=.031$, Cohen's d $=0.74$. Thus, the test for effectiveness of the bias explanation training was extra conservative. We found no other significant Condition differences on the background variables (Table 4.2) or pretest scores ( $p$ ranged from .839 to .061 , Cohen's d ranged from 0.07 to 0.64 ). 
Table 4.2. Comparison of participant characteristics between conditions

\begin{tabular}{lcccc}
\hline & $\begin{array}{c}\text { Experimental } \\
(n=18)\end{array}$ & $\begin{array}{c}\text { Control } \\
(n=19)\end{array}$ & & \\
\hline Independent t-tests to compare means & $\mathbf{M}(\mathbf{S D})$ & $\mathbf{M}(\mathbf{S D})$ & $\boldsymbol{t}$ & $\boldsymbol{p}$ \\
$\quad$ Age (years) & $45.3(8.7)$ & $48.1(10.9)$ & 0.85 & .400 \\
$\quad$ Teaching Experience (years) & $6.8(4.7)$ & $9.2(5.5)$ & 1.47 & .150 \\
& & & & \\
Chi-square tests to compare distributions & $\%$ & $\%$ & $\boldsymbol{X}^{2}$ & $\boldsymbol{p}$ \\
$\quad$ Sex - female & 66.7 & 21.1 & 7.84 & .005 \\
$\quad$ Level of Education - research-university & 66.7 & 52.6 & 1.22 & .270 \\
$\quad$ Previous Experience: & & & & \\
$\quad$ Reasoning biases $-1-20$ hours & 44.4 & 47.3 & 1.09 & .779 \\
$\quad$ Taking a logic course - yes & 22.2 & 57.9 & 4.88 & .027 \\
$\quad$ Teaching a logic course - yes & 11.1 & 31.6 & 0.002 & .969 \\
$\quad$ Taking a programming course -yes & 5.6 & 5.3 & 2.29 & .131 \\
$\quad$ Teaching a programming course - yes & 0.0 & 0.0 & - & - \\
\hline
\end{tabular}

Note. For $t$-tests degrees of freedom $(\mathrm{df})=35$ and for Chi-square tests $\mathrm{df}=1$, except Chi-square test on Previous Experience with reasoning biases for which $\mathrm{df}=3$. a Three levels: applied-university degree, research-university degree, and $\mathrm{PhD}$ ( $\mathrm{df}=1$ because $n=0$ for $\mathrm{PhD}$ ). ${ }^{\mathrm{b}}$ Eight response categories: 0 hours, 1-20 hours, 21-40 hours, 41-60 hours, 61-70 hours, 71-80 hours , 81-100 hours, and $>100$ hours ( $\mathrm{df}=3$ because $n=0$ for the four categories in italics).

\subsubsection{BIAS EXPLANATIONS: LEARNING TASKS}

Table 4.3 (top panel) shows the model statistics of the multilevel analyses testing whether the explanation training improved teachers' performance on Learning Tasks. Figure 4.3 (left panel) graphically displays the results of the Test-Occasion $\times$ Condition interaction (M3). As the figure illustrates, teachers in both conditions received on average about half of the maximum score for their provided explanations at the pretest (experimental: $\mathrm{M}=9.8, \mathrm{SD}=$ $3.9,95 \%$ CI $[7.9,11.8]$; control: $\mathrm{M}=10.1, \mathrm{SD}=4.2,95 \% \mathrm{CI}[8.1,12.1])$. After the training, however, the two conditions showed a divergent pattern. The experimental condition showed improved performance (compared to pretest) at both the immediate posttest $(\mathrm{M}=13.0, \mathrm{SD}$ $=5.7,95 \% \mathrm{CI}[10.2,15.8])$ and the delayed posttest $(\mathrm{M}=12.8, \mathrm{SD}=4.6,95 \% \mathrm{CI}[10.5$, 15.1]). The control condition, on the other hand, did not show improved performance (or even became worse) at immediate posttest $(\mathrm{M}=8.6, \mathrm{SD}=5.5,95 \%$ CI $[5.9,11.2])$. Yet, surprisingly, the control condition did improve from the pretest to delayed posttest $(M=11.9$, $\mathrm{SD}=4.0,95 \% \mathrm{CI}[10.0,13.8])$. The multilevel analyses confirmed the observed pattern. That is, the model including a cross-level interaction between Test Occasion and Condition (M3) fitted the data better than models including no predictors (M1) or merely Test Occasion as predictor (M2), M1-M2: $\Delta \chi^{2}(2)=9.82, p=.007$; M2-M3: $\Delta \chi^{2}(3)=13.46, p=.004$. In line with the descriptives, the interaction indicated a significant difference in pretest to immediate posttest gains favoring the experimental condition over the control condition, but there were 
no differences between conditions in gains from pretest to delayed posttest, Immediate $\times$ Condition: $t(103)=3.43 p<.001$; Delayed $\times$ Condition: $t(103)=0.84, p=.401 . \mathrm{R}_{2}{ }_{2}$ indicated that Condition explained only $1.7 \%$ extra variability between teachers' performance on Learning Tasks over and above Test Occasion. This small effect was not surprising given the non-significant differences at both pretest and delayed posttest. A post-hoc comparison between the two conditions at the immediate posttest yielded a performance difference with a large effect size, $t(35)=2.40, p=.022$ Cohen's $d=0.79$.

Table 4.3. Effect of the explanation training on teachers' performance on learning tasks and transfer tasks

\begin{tabular}{|c|c|c|c|}
\hline & $\begin{array}{c}\text { M1: } \\
\text { intercept only } \\
\text { (no predictors) }\end{array}$ & $\begin{array}{c}\text { M2: } \\
\text { effect of } \\
\text { Test Occasion }\end{array}$ & $\begin{array}{c}\text { M3: } \\
\text { effect of } \\
\text { explanation } \\
\text { training }\end{array}$ \\
\hline \multicolumn{4}{|l|}{ Learning Tasks } \\
\hline Fixed part & Coefficient (s.e.) & Coefficient (s.e.) & Coefficient (s.e.) \\
\hline Intercept & $11.01(0.66)^{\star \star}$ & $9.97(0.78)^{\star \star}$ & $10.11(1.05)^{\star \star}$ \\
\hline Immediate & & $0.76(0.74)$ & $-1.53(0.95)$ \\
\hline Delayed & & $2.35(0.74)^{\star}$ & $1.79(0.95)$ \\
\hline Condition & & & $-0.27(1.50)$ \\
\hline Immediate $\times$ Condition & & & $4.69(1.37)^{\star \star}$ \\
\hline Delayed $\times$ Condition & & & $1.16(1.37)$ \\
\hline \multicolumn{4}{|l|}{ Random part } \\
\hline Occasion and error variance $\left(\sigma^{2} e\right)$ & $11.59(3.41)$ & $10.15(3.19)$ & $8.66(2.94)$ \\
\hline Teacher variance $\left(\sigma^{2} u\right)$ & $11.95(3.46)$ & $12.43(3.53)$ & $12.22(3.50)$ \\
\hline Deviance & 639.14 & 629.32 & 615.86 \\
\hline \multicolumn{4}{|l|}{ Transfer Tasks (log transformed) } \\
\hline Fixed Part & Coefficient (s.e.) & Coefficient (s.e.) & Coefficient (s.e.) \\
\hline Intercept & $0.36(0.04)^{\star \star}$ & $0.13(0.06)^{\star}$ & $0.22(0.08)^{\star}$ \\
\hline Immediate & & $0.28(0.07)^{\star \star}$ & $0.10(0.09)$ \\
\hline Delayed & & $0.40(0.07)^{\star \star}$ & $0.31(0.09)^{\star \star}$ \\
\hline Condition & & & $-0.18(0.11)$ \\
\hline Immediate $\times$ Condition & & & $0.36(0.13)^{*}$ \\
\hline Delayed $\times$ Condition & & & $0.17(0.13)$ \\
\hline \multicolumn{4}{|l|}{ Random part } \\
\hline Occasion and error variance $\left(\sigma^{2}{ }_{e}\right)$ & $0.12(0.35)$ & $0.08(0.29)$ & $0.07(0.27)$ \\
\hline Teacher variance $\left(\sigma^{2} u 0\right)$ & $0.03(0.18)$ & $0.05(0.22)$ & $0.05(0.22)$ \\
\hline Deviance & 103.67 & 73.44 & 65.79 \\
\hline
\end{tabular}

Note. Test Occasion is dummy-coded in two dummies: Immediate ( $0=$ pretest, $1=$ immediate posttest $)$ and Delayed $(0=$ pretest, $1=$ delayed posttest $)$. Condition coded $0=$ control, $1=$ experimental. ${ }^{*} p<.05,{ }^{* *} p<.001$. 


\section{CHAPTER 4}

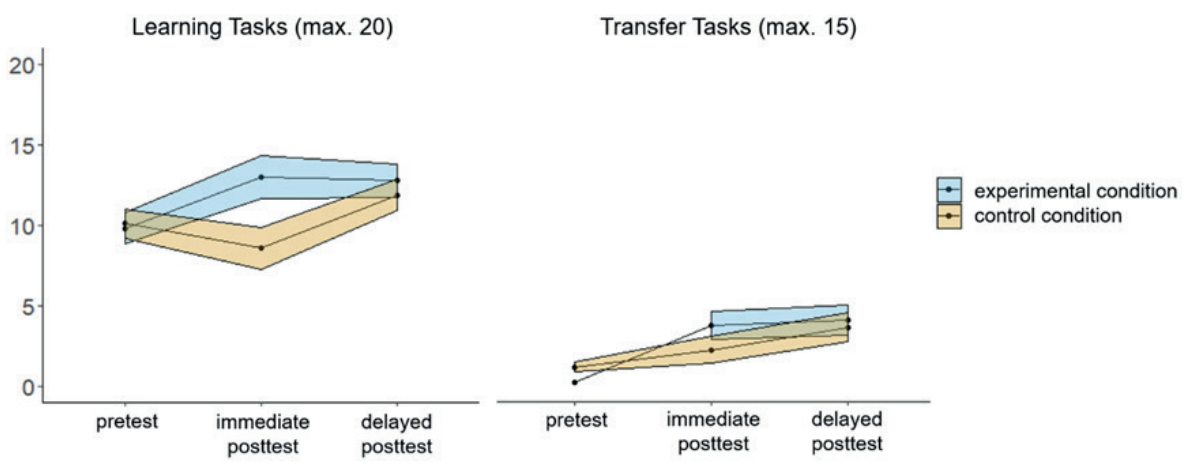

Figure 4.3. Plotted interaction effects between Test Occasion and Condition. Error bands indicate standard error.

Mental Effort. Across the test occasions, the average perceived mental effort invested in the learning tasks seemed to decrease slightly within the experimental condition, whereas it slightly increased within the control condition (see also Figure S4.1 in the Supplementary Material). This trend was, however, not further confirmed by the multilevel analyses, which indicated no significant differences in invested mental effort in the learning tasks between conditions or measurement occasions, with a grand mean of 5.0, $\mathrm{SD}=1.5,95 \% \mathrm{CI}[4.7,5.2]$, M1-M2: $\Delta \chi^{2}(2)=0.85, p=.655 ; \mathrm{M} 1-\mathrm{M} 3: \Delta \chi^{2}(5)=6.05, p=.302$.

In sum, in line with our first hypothesis we found a large benefit of the explanation training on teachers' bias explanations on Learning Tasks compared to the control condition, but - in contrast to our second hypothesis - this difference had disappeared two weeks later at the delayed posttest. Both conditions did perform better at delayed posttest than at pretest. Surprisingly, not so much the lack of a sustained intervention effect but rather a seemingly spontaneous performance improvement in the control condition from immediate to delayed posttest resulted in non-significant differences between the conditions. Consistent with previous research (Heijltjes et al., 2014, 2015; Janssen, Mainhard, et al., 2019; Van Peppen et al., 2018), participants attained improved performance without increasing their effort investment as prior to training (i.e., higher performance efficiency).

\subsubsection{BIAS EXPLANATIONS: TRANSFER TASKS}

Table 4.3 (bottom panel) and Figure 4.3 (right panel) show the results of the multilevel analyses on Transfer Tasks. As Figure 4.3 illustrates, both conditions followed a similar pattern. Pretest explanations were poor (experimental: $\mathrm{M}=0.2, \mathrm{SD}=0.9,95 \% \mathrm{CI}[-0.2,0.7]$; control: $\mathrm{M}=1.2, \mathrm{SD}=1.7,95 \% \mathrm{CI}[0.3,2.0])$, yet both conditions improved at the immediate posttest (experimental: $\mathrm{M}=3.7, \mathrm{SD}=3.7,95 \% \mathrm{CI}[1.9,5.5]$; control: $\mathrm{M}=2.2, \mathrm{SD}=3.7$, $95 \% \mathrm{CI}[0.4,4.0]$ ) and at the delayed posttest (experimental: $\mathrm{M}=4.1, \mathrm{SD}=4.4,95 \% \mathrm{CI}$ 
$[1.9,6.2]$; control: $\mathrm{M}=3.6, \mathrm{SD}=3.7,95 \% \mathrm{CI}[1.8,5.4])$. The analyses (Table 4.3) confirmed that there were no differences between conditions. The model including Test Occasion as predictor (M2) fitted the data better than an empty model (M1), M1-M2: $\Delta \chi^{2}(2)=30.23$, $p<.001$. In line with the descriptives, both conditions improved from pretest to immediate posttest, $t(106)=4.18, p<.001$, and from pretest to delayed posttest, $t(106)=5.95, p<.001$. Yet, adding a Test-Occasion $\times$ Condition effect (M3) did not further improve the model fit, M2-M3: $\Delta \chi^{2}(3)=7.65, p=.054$.

Mental Effort. The seeming trend towards decreasing mental effort investment across test occasions in the experimental condition and increasing effort investment in the control condition (see Figure S1) was not confirmed by the multilevel analyses, which indicated no significant differences in invested mental effort in the transfer tasks between conditions and test occasions, with a grand mean of $5.2, \mathrm{SD}=1.5,95 \% \mathrm{CI}[4.9,5.5], \mathrm{M} 1-\mathrm{M} 2: \Delta \chi^{2}(2)=2.91$, $p=.234 ; \mathrm{M} 1-\mathrm{M} 3: \Delta \chi^{2}(5)=8.88, p=.114$.

In sum, all teachers improved on the Transfer Tasks after training, and this performance improvement did not require additional effort investment (i.e., higher performance efficiency).

\subsubsection{EXPLORING TEACHERS' EXPLANATIONS}

As shown in Table 4.1, teachers' explanations were scored on quality of bias explanation (no explanation, detection, information-lack explanation, or counter-example explanation) and on suggestion for improvement (no suggestion, reservation suggestion, or required-information suggestion). We explored the frequencies of the assigned codes to teachers' provided explanations ${ }^{8}$. In general, the results endorsed the patterns found with the statistical analyses and, additionally, they showed how teachers explained the students' biases before and after (explanation) training.

Learning Tasks. Figure 4.4 shows an overview of the averaged code frequencies on the Learning Tasks. Conditions exhibited a similar pattern at pretest, a different pattern at immediate posttest, and again a more or less similar pattern at delayed posttest. Prior to intervention (pretest), about a quarter of the teachers did not provide correct statements about the students' logic (no explanation: $\mathrm{M}=25.7 \%, \mathrm{SD}=16.0$ ). Another quarter did recognize what element in the students' argument was logically invalid but did not (correctly) explain why it was invalid (detection: $\mathrm{M}=23.65 \%, \mathrm{SD}=8.9$ ). Nevertheless, already $50.7 \%$ ( $\mathrm{SD}=$ 10.9 ) of the teachers explained why the students' conclusion was logically invalid, either by pointing out a lack of information $(\mathrm{M}=6.8 \%, \mathrm{SD}=9.0)$ or by providing a counter-example $(\mathrm{M}$ $=43.9 \%, \mathrm{SD}=13.8)$. Also, $43.2 \%(\mathrm{SD}=12.9)$ provided students with a concrete suggestion

\footnotetext{
${ }^{8}$ We first examined the frequencies of the assigned codes per learning and transfer task (explanation and suggestion category), Test Occasion, and Condition separately (see Tables S4.4 -S4.7 in the Supplementary Material). By and large we found similar patterns applying to the tasks within one measurement occasion and experimental condition. For conciseness ( 7 tasks $\times 2$ code categories $\times 3$ test occasions $\times 2$ experimental conditions $=84$ frequency patterns) we therefore we averaged the frequency patterns.
} 


\section{CHAPTER 4}

for improvement; $6.1 \%(\mathrm{SD}=5.6)$ suggested to place a reservation to the conclusion and $37.2 \%(\mathrm{SD}=14.2)$ explained what information would allow the students to draw their original conclusion.

Teachers who followed the explanation training improved their bias explanations at immediate posttest. The number of teachers that failed to mention anything about logic (no explanation) decreased, whereas the number of teachers providing the highest-rated explanation (counter-example) and suggestion (required-information) increased (see Figure 4.4). The control condition still showed a rather similar pattern to the pretest. At delayed posttest, the improved explanation pattern in the experimental condition was sustained. Remarkably, however, the control condition showed almost the same (improved) pattern at this point.

Finally, at the pretest, $20.8 \%(\mathrm{SD}=11.5)$ of the teachers in the experimental condition used invalid logic in their own explanations as compared to $13.2 \%(\mathrm{SD}=12.5)$ in the control condition. In both conditions, the number of teachers using invalid logic decreased from pretest to immediate posttest (experimental: $\mathrm{M}=5.6 \%, \mathrm{SD}=4.5$; control: $\mathrm{M}=9.2 \%, \mathrm{SD}=$ 9.0) and from pretest to delayed posttest (experimental: $\mathrm{M}=11.1 \%, \mathrm{SD}=9.1$; control: $\mathrm{M}=$ $1.3 \%, \mathrm{SD}=2.6$ ).

Transfer Tasks. For both conditions, a similar explanation pattern for the Transfer Tasks emerged (see Figure 4.5). At the pretest, the large majority of teachers failed to provide an accurate explanation $(\mathrm{M}=91.9 \%, \mathrm{SD}=4.7)$, with only a few teachers who provided a good explanation of the reasoning bias (information-lack explanation: $\mathrm{M}=2.7 \%, \mathrm{SD}=2.7$; counter-example explanation: $\mathrm{M}=5.4 \% \mathrm{SD}=2.7$ ). Virtually none of the teachers provided a correct suggestion for improvement. After the CT-training, the number of teachers providing an accurate explanation (information-lack or counter-example) and suggestion (reservation or required-information) increased both at immediate posttest (explanation: $\mathrm{M}=22.5 \%$, SD $=8.7$; suggestion: $\mathrm{M}=14.4 \%, \mathrm{SD}=1.6$ ) and at delayed posttest (explanation: $\mathrm{M}=37.8 \% \mathrm{SD}$ $=7.2$; suggestion: $\mathrm{M}=10.8 \%, \mathrm{SD}=0.0$ ). Finally, just as with Learning Tasks, teachers' use of invalid logic in their own explanations decreased from pretest (experimental: $M=22.2 \%$, $\mathrm{SD}=5.6$; control: $\mathrm{M}=14.0 \%, \mathrm{SD}=11.0$ ) to immediate posttest (experimental: $\mathrm{M}=9.3 \%$, $\mathrm{SD}=3.2$; control: $\mathrm{M}=12.3 \%, \mathrm{SD}=11.0$ ) and to delayed posttest (experimental: $\mathrm{M}=13.0 \%$, $\mathrm{SD}=3.2$; control: $\mathrm{M}=8.8 \%, \mathrm{SD}=11.0$ ).

\subsubsection{TEACHING ATITUDES}

We observed that teachers' average ratings of the relevance of teaching CT and their competence in teaching it were quite high and quite similar in both conditions and constant across all test occasions, with a grand mean of $4.7(\mathrm{SD}=0.6,95 \% \mathrm{CI}[4.6,4.8]$ for Perceived Relevance and of 4.1 (SD $=0.7,95 \%$ CI $[4.0,4.2]$ ) for Perceived Competence (see also Figure S4.2 in the Supplementary Material). The multilevel analyses indeed confirmed that there were no significant changes; Perceived Relevance: M1-M2: $\Delta \chi^{2}(2)=4.91, p=.086$; M1-M3: $\Delta \chi^{2}(5)=11.07, p=.050$; Perceived Competence: M1-M2: $\Delta \chi^{2}(2)=4.28, p=.118$; M1-M3: $\Delta \chi^{2}(5)=8.08, p=.152$. 


\section{TRAINING CRITICAL THINKING TEACHING SKILLS}

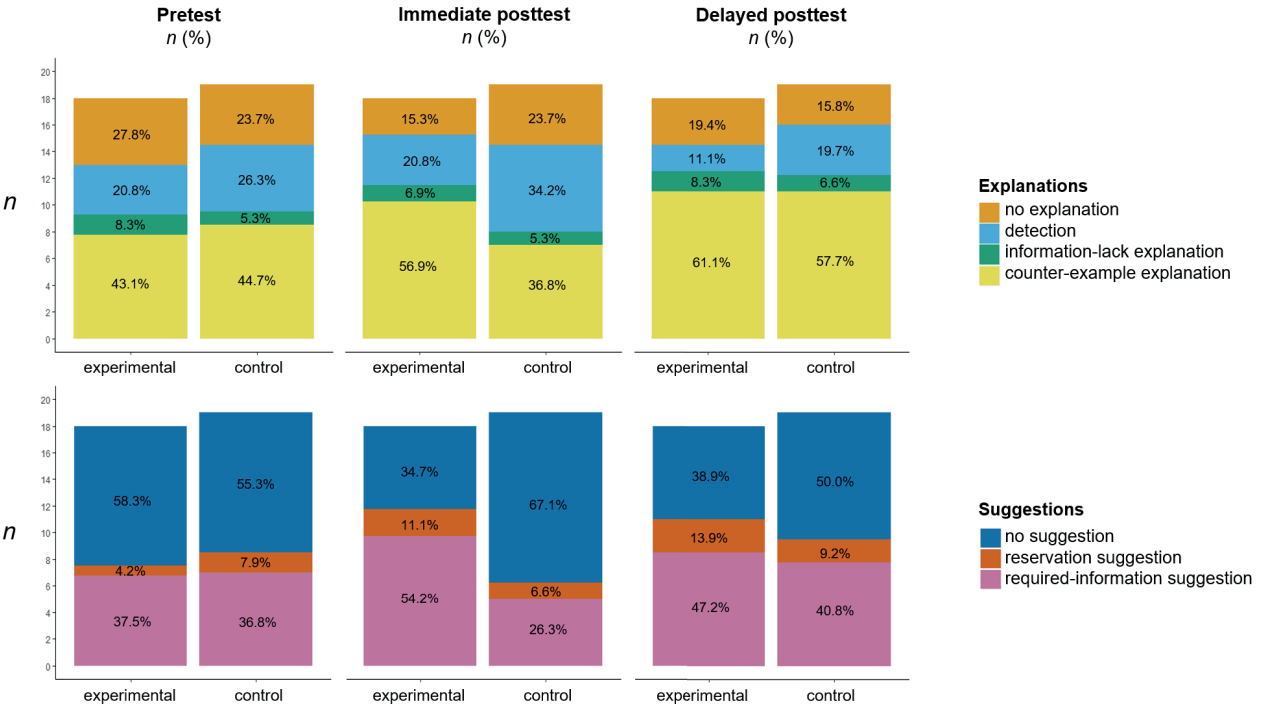

Figure 4.4. Overview of the code frequencies, averaged across Learning Tasks, per Test Occasion and Condition.
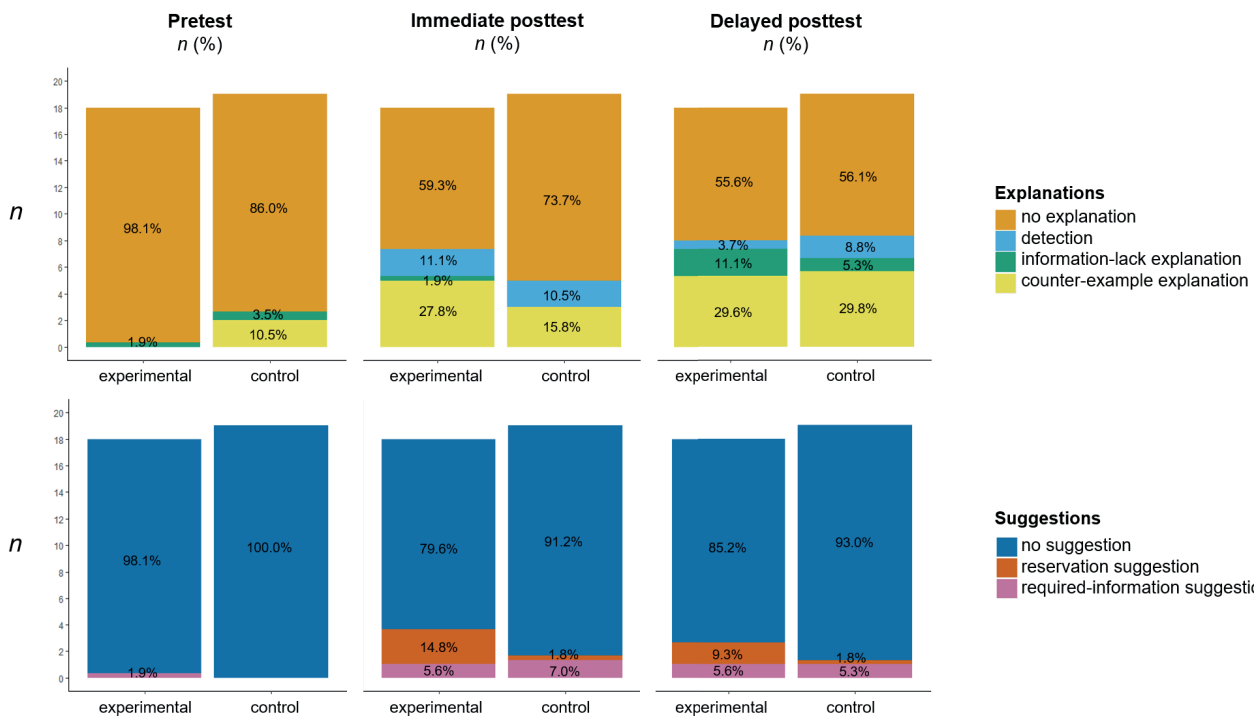

Suggestions

no suggestion

reservation suggestion

required-information suggestion

Figure 4.5. Overview of the code frequencies, averaged across Transfer Tasks, per Test Occasion and Condition. 


\subsection{DISCUSSION}

The aim of the present study was to gain more insight in how to foster higher education teachers' ability to explain biases in students' reasoning, a crucial skill for teaching CT. To this end, we experimentally tested the impact of extending CT training focused on avoiding biases (Janssen, Mainhard, et al., 2019) with an online 'bias explanation training'. In line with our first hypothesis, teachers who received explanation training improved their explanations of biases they had practiced with at an immediate posttest (i.e., learning), whereas teachers in the control condition did not. The experimental condition maintained this improvement on a delayed posttest two weeks later, but - in contrast to our second hypothesis - the control condition had also improved, seemingly spontaneously. The explanation training had no effect on explanations of biases teachers had not practiced with (transfer), on teachers' perceived invested effort in explaining biases, or on teachers' perceived relevance of and perceived competence in teaching $\mathrm{CT}$.

\subsubsection{LEARNING EFFECT OF BIAS EXPLANATION TRAINING}

The results showed a large immediate effect of the explanation training on learning. First, the test of effectiveness was extra conservative since the experimental condition seemed less skilled in logical reasoning prior to the training than the control condition (i.e., less experience in logical reasoning and lower pretest performance on Transfer Tasks). Despite that disadvantage, the experimental condition significantly outperformed the control condition immediately after following the explanation training. This improvement was attained with a similar amount of effort as prior to training, which seems to point to acquired efficiency in explaining the biases (Hoffman \& Schraw, 2010; Paas \& Van Merriënboer, 1993). Further explorations indicated that $63.8 \%$ of the teachers who had followed the CT-training plus explanation training (experimental condition) correctly explained why the students' conclusion was logically invalid and that $65.3 \%$ provided a concrete suggestion for improvement, compared to $42.1 \%$ and $32.9 \%$, respectively, of teachers who only received CT-training (the control condition). The number of teachers who received the highest rated explanations (counter-example) and suggestions (required-information) increased slightly (with about 15\%) in the experimental condition whereas it did not increase at all in the control condition. Thus, although there was still room for improvement, the fact that almost all teachers showed at least some improvement in their explanations on the immediate posttest without investing extra effort, and that a few even managed to arrive at very good explanations after only one training session, is promising.

Whereas the learning effect remained over time in the sense that the experimental condition still scored better on explaining the practiced biases at delayed posttest than at pretest, there was no longer a significant difference with the control condition at the delayed posttest. We should note that in contrast to the immediate posttest, the delayed posttest was only marginally reliable and thus has higher chance to be influenced by measurement errors. Assuming we can trust these findings despite this reliability issue, the question is why the 
control condition suddenly improved their explanation performance on the delayed test. Although we have no information on this, one possible explanation is that they talked to and learned from colleagues who had received the explanation training (given the random assignment, each session had teachers who did and did not receive explanation training).

\subsubsection{TRANSFER EFFECT OF BIAS EXPLANATION TRAINING}

We found no immediate or delayed effect of the explanation training on transfer performance. Both conditions improved their explanations (again without requiring more effort; i.e., higher performance efficiency). However, the average performance scores were still rather low, and almost none of the teachers could provide adequate suggestions for improving the logic of the students' argument. The observed improvement in both conditions and the lack of added value of the explanation training is interesting, as it seems to suggest that the CT-training, which focused on teachers' own skills to avoid bias, was sufficient to achieve transfer to the teaching skill of explaining these biases. This is rather surprising given the difficulty level of the transfer tasks (as indicated by the floored pretest performance) and because previous studies demonstrated that transfer is so difficult to achieve (Heijltjes et al., 2014, 2015; Janssen, Mainhard, et al., 2019; Van Peppen et al., 2018). One potential explanation is that the transfer (i.e., Wason selection) task had been explained during the CT-training (so all teachers might have remembered this). Previous studies measured transfer on tasks addressing biases that were not previously instructed that explicitly (cf. Heijltjes et al., 2015; Heijltjes, Van Gog, Leppink, et al., 2014; Van Peppen et al., 2018). Another potential explanation is that the four logic rules underlying the syllogism tasks (which were also relevant for the Wason tasks) were displayed on the board during the posttests (which may have helped teachers to map these onto the Wason tasks). In sum, although the improvement in performance on the transfer tasks is positive, there is substantial room for improvement and it is not fully clear how to achieve that; explanation training did not seem to have added value for transfer.

\subsubsection{ATITUDES TOWARDS TEACHING CRITICAL THINKING}

In line with our previous study (Janssen, Mainhard, et al., 2019), all participating teachers already perceived the teaching of CT as highly relevant prior to the training. As such, it is not surprising that the training did not affect perceived relevance. This finding of high perceived relevance is interesting though, because in our previous studies, participation was voluntary, which opened the door to self-selection bias (Janssen, Mainhard, et al., 2019; Janssen, Meulendijks, et al., 2019). In contrast, in the present study we did not expect participants to be positively biased, because the training was now a mandatory part of a professional development program. Hence, our findings confirm that teachers (from this university of applied sciences) feel that teaching CT is important.

We found no significant changes over time or between conditions in teachers' perceived competence in teaching CT either. Thus, despite the fact that teachers became better at an important skill for teaching CT, they not did feel more competent in teaching CT. Attaining 
an increased feeling of competence in teaching such a complex skills as CT may, however, require more time and opportunity for practice than was available in the current training setting (cf. research on science teaching; Van Aalderen-Smeets \& Walma van der Molen, 2015). Interesting for future research would be to further investigate teachers' calibration with regard to teaching CT (i.e., perceived competence vs. actual competence in teaching CT).

\subsubsection{LIMITATIONS AND FUTURE DIRECTIONS}

One limitation in this study concerns the sample size. The size was not small for an experiment in an actual educational setting with teachers participating in the context of a professional development program, and was still large enough to pick up medium to large training effects, but smaller effects could not be captured with the current sample. Related to this, the sample size was too small to obtain precise Cronbach's alpha (and other reliability) coefficients (Charter, 2003). Because we cannot be fully sure how (un)reliable our measures were, we cannot know how adequate or sensitive the instruments classified individuals' scores from low to high, which again reduces the chance to pick up more subtle changes accurately. The low reliability of our pretest measures can at least partly be explained by a lack of prior knowledge of the participants, resulting in more inconsistent answers or zero scores (which in turn result in low variance). The marginal reliability of the Learning Tasks at delayed posttest, however, is somewhat harder to explain other than by the small sample size. Nevertheless, even when taking these limitations into account the current study still provides valuable new insights in how to equip teachers for teaching CT, especially given the paucity of (experimental) research on how teachers reason about their students' biased reasoning and on how to improve it.

Our findings imply that it is possible to foster teachers' ability to explain biases in their students' reasoning. Given the importance of this teaching skill for students to improve their CT-skills, this is promising. Relevant next steps would be to also investigate the effectiveness of such a training in other teaching domains and with other biases. Our training was situated in a Safety and Security Management education program and focused on two common biases in logical reasoning. There is, however, an endless list of existing cognitive biases and it might differ per teaching domain which biases are most important to avoid. Broader implementation of bias explanation training therefore requires knowledge on what particular reasoning biases are most important for teachers to be trained in, hereby distinguishing between biases relevant for each teaching domain and domain-specific biases. Another important question is how (well) the trained explanation skills transfer to teachers' actual feedback to their students' reasoning in assignments or in the classroom and whether this would improve students' performance. Although our study materials were based on actual student papers, explaining logical reasoning biases in 'real' student papers is more difficult than it was at our test tasks (i.e., actual papers longer and typically contain more ill structured arguments). Ultimately, we need to know how to implement teacher training on CT in an effective way, such that teachers endorse the specific learning goals and that the learning environments are set up (e.g., enough support and time) to achieve these goals (Van Veen et al., 2012). 


\subsubsection{CONCLUSION}

In sum, based on our study results we can conclude that it possible to foster teachers' ability to explain students' logical reasoning biases. This is relevant because being able to explain biases is necessary to provide adequate feedback to students, which in turn is essential for students' CT-skills acquisition. 


\section{PART 2}

\section{Cognitive mechanisms}

and dispositions underlying biased reasoning 

Chapter 5

Recognizing biased reasuning:

Conflict detection during

decision-making and decision-evaluation

An adapted version of this chapter is submitted for publication as:

Janssen, E. M., Velinga, S.B., De Neys, W., \& Van Gog, T. (submitted). Recognizing biased reasoning: Conflict detection during decision-making and decision-evaluation. Manuscript submitted for publication.

\section{Acknowledgement of author contributions:}

EJ, SV, and TVG designed the study, SV recruited participants and collected the data, EJ and SV analyzed the data, EJ drafted the manuscript, all authors contributed to critical revision of the manuscript, TvG supervised the study. Additional note: Tim Mainhard checked the data package. 


\section{ABSTRACT}

Although it is well established that our thinking is often biased, the precise cognitive mechanisms underlying this bias are still debated. The present study builds on recent research showing that biased reasoners often seem aware that their reasoning is incorrect; they show robust signs of error or "conflict" detection. One important shortcoming in this research, however, is that the conflict detection effect has only been studied with classical, arguably non-realistic, heuristics-and-biases tasks in which participants have to make a decision (i.e., problem solving) involving fairly trivial consequences. Therefore, the present study $(N=159)$ investigated whether conflict detection also occurs during tasks with more realistic scenarios involving decision-making (problem solving) and decision-evaluation (vignettes on decisions of others). We analyzed participants' conflict detection sensitivity on confidence, response time, and confidence response time measures. Results showed that conflict detection (as indicated by decreased confidence) also occurred on the more realistic tasks used in the present study, during both decision-making and decision-evaluation. The response time indices appeared to be less reliable measures of conflict detection. These findings are especially relevant for (studying) reasoning in contexts in which recognizing reasoning errors is important (e.g., in medicine where doctors have to evaluate initial diagnoses of others, or in education where teachers have to detect and give feedback on biases in their students' reasoning).

Keywords. reasoning and decision-making; decision-evaluation; heuristics and biases; conflict detection. 


\subsection{INTRODUCTION}

Every day, people make countless decisions and the vast majority is made effortlessly, without deliberate thought. While this is highly adaptive (we would be exhausted if we had to think through each and every decision) and usually yields good decisions, it can also lead to biases in reasoning (Kahneman, 2011; Stanovich et al., 2016). For instance, consider the following reasoning problem:

In a study 1000 people were tested. Among the participants there were 5 dentists and 995 rock singers.

Stan is a randomly chosen participant of the study. Stan is 36 . He married his college sweetheart after graduating and has two kids. He doesn't drink or smoke but works long hours.

What is most likely?

Stan is a dentist

Stan is a rock singer

Because the description of Stan fits with people's stereotypes of a dentist, most people indicate that Stan is most likely a dentist (cf. $80 \%$ in a university student sample, see De Neys, Cromheeke, \& Osman, 2011; and 60\% in a North-American Mechanical Turk sample, see Frey, Johnson, \& De Neys, 2018). According to principles of statistical probability, however, this conclusion is not correct. The description of Stan indeed fits the image of a dentist, but could also apply to a rock singer and - importantly - since the large majority of the study's participants are rock singers it is much more likely that Stan is a rock singer than a dentist. This biased conclusion is referred to as "base-rate neglect", and base-rate neglect problems such as this one are illustrative of the classical "heuristics-and-biases tasks". These tasks are widely used to demonstrate that human judgment is often based on fast intuitions or "heuristic" thinking rather than on more deliberate reasoning (Kahneman, 2011). In the example, people tend to make a probability estimation based on a representativeness heuristic (telling them whether the description is more representative of a dentist or rock singer), which leads to a statistical base-rate neglect bias in their estimation.

Decades of reasoning and decision-making studies have proven that people typically perform very poorly on a wide range of heuristics-and-biases tasks (Evans \& Over, 1996; Kahneman, 2011). Although biases are inherent to human cognition and are often relatively innocent, there are many situations in which biased decisions can have serious consequences. For example, when a judge misinterprets evidence based on intuitive stereotypical associations (Eberhardt, Davies, Purdie-Vaughns, \& Johnson, 2006; Thompson \& Schumann, 1987), when a doctor makes a diagnostic error due to exposure to popular media information about a disease (Schmidt et al., 2014), when investors make bad investment decisions based on the mere familiarity of a stock (Oster \& Koesterich, 2013), or when parents decide not to 
vaccinate their children because of rare but highly publicized instances in which vaccines have failed (Smith, 2017).

Although it is well established that our thinking is often biased, the precise cognitive mechanisms underlying this bias are still debated. Until recently, influential scholars in the field suggested that most people perform poorly on heuristics-and-biases tasks because they do not recognize that their intuitive heuristic response is at conflict with logical or probabilistic principles (Evans \& Stanovich, 2013; Kahneman, 2011). Put differently, it was assumed that biased reasoners are completely unaware of the error in their reasoning. Interestingly, however, recent studies have started to show that, even though they make a biased decision, most biased reasoners do show at least some sensitivity to the conflict between their heuristic response and logical considerations (De Neys \& Pennycook, 2019). These studies typically compared individuals' responses on reasoning problems that - as in the "Stan" problem above - prime a heuristic response which is incongruent with logical principles (i.e., conflict problems) to responses on problems that prime a heuristic response which is congruent with the logical principles (i.e., no-conflict problems). A no-conflict version of the "Stan" problem above would refer to a study sample of 995 dentists and 5 rock singers, so that the most likely option is congruent with the prompted stereotype. In other words, conflict and no-conflict problems trigger the exact same heuristic response (e.g., Stan is probably a dentist), but only on the no-conflict problem is this heuristic response also the correct response. Not surprisingly, almost everyone solves no-conflict problems correctly (De Neys et al., 2011; Frey et al., 2018).

Interestingly, even though most people give the same heuristic responses to both conflict problems and no-conflict problems, they process the two problems differently. People take significantly longer to enter their (incorrect) heuristic response on conflict problems than they do to enter their (correct) heuristic response on no-conflict problems (e.g., Bonner \& Newell, 2010; De Neys \& Glumicic, 2008). They are also less confident about their (incorrect) responses to conflict problems, compared to their (correct) responses to no-conflict problems (e.g., De Neys et al., 2011; Gangemi et al., 2015). In other words, biased reasoners do show sensitivity to the logical conflict. This conflict detection effect, as indicated by confidence ratings, response times, or confidence response times (i.e., the time taken to provide a confidence rating) has been found across a wide variety of classical heuristics-and-biases tasks (Bago \& De Neys, 2017; De Neys, 2014; Frey et al., 2018; Mevel et al., 2015; Pennycook et al., 2015a; Stupple et al., 2013; although there are also a few studies that found no evidence for conflict detection: Ferreira et al., 2016; Mata et al., 2017; Pennycook et al., 2012).

Despite the increasing number of studies showing that most biased reasoners show sensitivity to their reasoning errors, research on the conflict detection effect is still in its formative stages and the effect requires further investigation (De Neys, 2012, 2014; De Neys $\&$ Pennycook, 2019). One important shortcoming is that the conflict detection effect has only been studied with classical heuristics-and-biases tasks, like the base-rate neglect problem above. This is problematic because, in the end, we want to know how biased reasoning occurs in everyday situations and - while effective for demonstrating bias - these classical tasks are arguably rather artificial (e.g., judging whether a person is most likely a dentist or rock singer 
is quite far removed from important real-world decisions with far-reaching consequences). In everyday situations, we are often confronted with (biased) conclusions or decisions made by others and have to decide for ourselves whether or not we agree with them. For example, when reading news articles, people are not asked to actively reason about the likelihood of a particular situation, but are confronted with a likelihood estimation made by someone else. When that estimation confirms a reader's own intuitive ideas, recognizing that it is biased is arguably just as difficult as making the estimation yourself. This ability to detect biases in texts reflecting the reasoning of others is very important in many contexts. For instance, in medicine where physicians often see patients after a referral and initial diagnosis by another doctor (Van den Berge et al., 2012) or in education where teachers have to detect and give feedback on biases in their students' reasoning (Janssen et al., 2019).

Thus, to improve our knowledge of biased reasoning in everyday situations, it is important to establish whether people would detect biased reasoning in decisions of others, and if not, whether they at least show signs of conflict detection. Detecting a conflict in your own versus another person's decision might involve similar cognitive mechanisms. In this case, failing to detect bias in reasoning of others would occur as frequently as failing to override bias in people's own reasoning, and, moreover, a similar conflict detection effect might apply. However, it could also be the case that the underlying mechanisms differ. For instance, research into argumentation suggests that people become more deliberative and critical when they have to judge the argumentation of others than when they themselves have to make a judgment (Mercier \& Sperber, 2011; Trouche et al., 2016). If this is the case, then people would be more likely to detect biases in reasoning of others than in their own, and possibly show even stronger signs of conflict detection. On the other hand, people typically agree with conclusions confirming their own ideas and beliefs (Markovits \& Nantel, 1989; Thompson \& Evans, 2012). Thus, if someone else's conclusion is in line with their own intuitive ideas and the related decision does not directly affect them, people might be less motivated to pay attention to someone else's reasoning. In that case, people would be less likely to detect biases in reasoning of others than in their own or to show signs of conflict detection.

\subsubsection{THE PRESENT STUDY}

In sum, it is both theoretically and practically relevant to investigate conflict detection in real-world scenarios and to establish whether people would detect biased reasoning in decisions of others, and if not, whether they at least show signs of conflict detection. Therefore, the present study explored the conflict detection effect on problem-solving tasks (i.e., decision-making) as well as on vignette tasks in which participants had to evaluate decisions made by others as described in short texts (i.e., decision-evaluation). In both the problem-solving tasks and the vignette tasks, we used more realistic (real-world) scenarios than in the classical heuristics-and-biased tasks that have been used thus far to study conflict detection. The study was explorative in nature; as mentioned earlier, it is hard to make a priori predictions on whether reasoning accuracy and conflict detection would differ or not between problem-solving and vignette tasks. We investigated conflict detection on three 
different response indices (cf. Frey et al., 2018): confidence, response time, and confidence response time. A lower confidence on incorrectly performed conflict tasks relative to correctly performed no-conflict tasks would point to conflict detection; a higher response time meaning slower answering - on incorrectly performed conflict tasks relative to correctly performed no-conflict tasks would point to conflict detection; and higher response time when making a confidence judgment on incorrectly performed conflict tasks relative to correctly performed no-conflict tasks would point to conflict detection.

\subsection{METHOD}

\subsubsection{PARTICIPANTS}

In total, 160 native Dutch-speaking participants were recruited on Prolific Academic (www. prolific.ac) and paid $£ 7.75$ for participation. One participant had to be excluded due to a technical error, leaving a final sample of 159 participants (108 males, 49 females, 2 indicated "other"; age: $\mathrm{M}=26.9$ years, $\mathrm{SD}=9.2$ ). In terms of educational background, $73.0 \%$ of the participants reported having obtained a higher education degree or being enrolled to obtain this degree, $9.4 \%$ a vocational education degree, and $17.6 \%$ a secondary education degree.

Data statement. All data is stored on an Open Science Framework (OSF) page for this project, see https://osf.io/k7uhs/?view_only=62ff2947f60743b4aa556e194cd0355a (anonymized view-only link for the purpose of masked review).

\subsubsection{MATERIALS}

Based on classical base-rate and conjunction problems (see e.g., De Neys et al., 2011; Frey et al., 2018), we designed a total of 24 new reasoning tasks (in Dutch): six base-rate problem-solving tasks (i.e., decision-making), six base-rate vignette tasks (i.e., decision-evaluation), six conjunction problem-solving tasks (i.e., decision-making), and six conjunction vignette tasks (i.e., decision-evaluation). The new tasks differed in several respects from the previously studied classical problems. Whereas classical heuristics-and-biases problems typically described short and simple situations in which the reasoning was quite far removed from important real-world decisions with far-reaching consequences, the current tasks described longer and more complex situations in which the required reasoning was always relevant for achieving a relevant goal (e.g., tackling companies committing fraud or deciding whether soups are likely to contain dangerous additives). In addition, next to the typical problem-solving tasks in which participants have to make a probability estimation themselves (i.e., decision-making), we introduced vignette tasks, in which the participants' job was to evaluate the reasoning of another person (i.e., decision-evaluation).

Base-rate problem-solving tasks (i.e., decision-making). Three out of the six base-rate problem-solving tasks were conflict tasks: the description and base-rates cued conflicting responses. The other three were no-conflict tasks in which the description and base-rates 
cued the same response. A translated example of a base-rate problem-solving task in conflict version is:

The Dutch government has recently made tackling fraud by companies one of the police's priorities. The police have received a list of 1000 companies that may be committing fraud. Further investigation has shown that 8 of these companies have committed fraud and that the remaining 992 companies have not committed fraud. However, certain information was lost during a reorganization. The police no longer know which companies have committed fraud. Van Been Ltd is a randomly chosen company that is on the police's list.

Van Been Ltd has a closed and competitive corporate culture. Its employees put a lot of effort into making big profits. The annual report also shows that the company has made a remarkably high profit in the past year. There is also a strikingly high number of fines that employees have received in company cars.

What is most likely?

Van Been Ltd committed fraud

Van Been Ltd did not commit fraud

As in the classical base-rate problems, the narrative description was designed to cue an intuitive response based, on a stereotype that is at odds with the base-rate information. All tasks had the same underlying structure of about the same word length, but a different cover story. Each task started with a sentence that introduced a particular situation, followed by two sentences including base-rate information, a sentence with additional information explaining the current situation, and a sentence introducing a randomly selected individual case. In the next paragraph, specific information about the selected individual case was presented, after which the participant had to indicate which of two possible situations was most likely ${ }^{1}$. To construct a no-conflict version, we simply changed the sentence including the base-rate information, so that the intuitively cued response was in line with the statistically most likely option (e.g., "Further investigation has shown that 992 of these companies have committed fraud and that the remaining 8 companies have not committed fraud).

Base-rate vignette tasks (i.e., decision-evaluation). Three out of the six base-rate vignette tasks were conflict tasks (heuristic decision by other is in conflict with base-rate) and three were no-conflict tasks (heuristic decision by other is in line with base-rate). Here is an example of the earlier base-rate problem-solving task in vignette format:

\footnotetext{
${ }^{1}$ Responses that were in line with the base-rates (i.e. selection of the largest group as most likely answer) were labeled as correct answers. In line with Frey et al. (2018), we used extreme base-rates (variations around 995 and 5) and moderate cues to minimize the concern developed by Gigerenzer, Hell, and Blank (1988) that when relying on a formal Bayesian approach, selection of the heuristic response should be considered normatively correct (see De Neys, 2014).
} 
The Dutch government has recently made tackling fraud by companies one of the police's priorities. The police have received a list of 1000 companies that may be committing fraud. Further investigation has shown that 8 of these companies have committed fraud and that the remaining 992 companies have not committed fraud. However, certain information was lost during a reorganization. The police no longer know which companies have committed fraud. One of the companies on the list, Van Been Ltd, stands out for the police because of a strikingly high number of fines that employees have received in company cars. Van Been Ltd has a closed and competitive corporate culture. Its employees put a lot of effort into making big profits. The annual report also shows that the company has made a remarkably high profit in the past year. The police have decided to start an official investigation into the company, because they estimate it more likely that Van Been bv has committed fraud than that Van Been bv has not committed fraud.

Is the estimation on which the police have based its decision correct?

Yes

No

As the example indicates, the vignette tasks were very similar to the problem-solving tasks, but differed on three aspects. First, instead of just presenting information about a randomly chosen individual case, the story explained that one individual case had caught the attention of one of the actors in the story. Second, a sentence was added in which the actor estimated the likelihood of two possible situations, on which a specific decision was based. Third, instead of indicating which of two possible situations was most likely (cf. problem-solving tasks), participants had to indicate whether the estimation on which the actor's decision was based, was correct. No-conflict versions were again constructed by switching the base-rate information.

Conjunction problem-solving tasks (i.e., decision-making). Of the six conjunction problem-solving tasks (see section 5.1 in the Supplementary Materials for an example of the classical conjunction problem), three were again conflict tasks and three were no-conflict tasks. An example of a conjunction problem-solving task in conflict version is:

In the past year, the Dutch Food and Consumer Product Safety Authority has investigated 10 brands of tomato soup to determine whether these contained dangerous additives or not. Immediately after the investigation, Heinz removed all its tomato soups from the store shelves, according to the company itself in order to improve the taste of the soup.

What is most likely?

Heinz wanted to improve the taste of the soup.

Heinz wanted to improve the taste of the soup and the soup contained dangerous additives.

The conflict above emerges because the cued stereotype (the soup contained dangerous 
additives) is in the conjunctive answer option, yet logically, the conjunction of any two probabilities can never be more likely than either of the conjuncts in isolation (formally: $p(\mathrm{~A} \& \mathrm{~B}) \leq p(\mathrm{~A}), p(\mathrm{~B}))$. In other words, the probability of Heinz wanting to improve the taste plus the soup containing dangerous additives can never be greater than merely the probability of Heinz wanting to improve the taste of the soup. Each task had about the same word length and was structured as follows: It started with a sentence that introduced a particular situation. Next, an action by a person or institution was described. The person or institution always provided an unlikely explanation for this action, after which participants had to indicate which of two possible situations in the answering option was most likely ${ }^{2}$. Following Frey et al. (2018), to construct a no-conflict version we changed the person's or institution's provided unlikely explanation into a likely explanation (e.g., "Immediately after the investigation, Heinz removed all its tomato soups from the store shelves, according to the company itself because the soup contained dangerous additives") and replaced the unlikely explanation in the non-conjunctive answering option with the likely explanation:

What is most likely?

The soup contained dangerous additives.

The soup contained dangerous additives and Heinz wanted to improve the taste of the soup.

Conjunction vignette tasks (i.e., decision-evaluation). Three out of the six conjunction vignette tasks were conflict tasks and three were no-conflict tasks. The conjunction problem-solving task above in vignette format is:

In the past year, the Dutch Food and Consumer Product Safety Authority has investigated 10 brands of tomato soup to determine whether these contained dangerous additives or not. Immediately after the investigation, Heinz removed all its tomato soups from the store shelves, according to the company itself in order to improve the taste of the soup. However, according to an investigative journalist of the Volkskrant [Dutch news paper], it is more likely that Heinz not only wanted to improve the taste of the soup but that the soup also contained dangerous additives.

Is the estimation of the investigative journalist of the Volkskrant correct?

Yes

No

\footnotetext{
${ }^{2}$ People have the tendency to choose the answer that contains the stereotypical description, irrespective of whether this is the conjunctive or non-conjunctive answer option (Tversky \& Kahneman, 1983). Although, it is possible that the conjunction of two probabilities is equally large as one of the two in isolation, it can never exceed the probability of either one in isolation. Therefore, the conjunctive answering option can never be more likely than the non-conjunctive one. Hence, in this reasoning situation one should normatively always choose the non-conjunctive statement.
} 
As the examples indicate, the conjunction vignette tasks differed from the problem-solving tasks on two aspects. First, a sentence was added in which a new actor was introduced (e.g., an investigative journalist), who made a decision about the likelihood of two possible situations. Second, instead of indicating which of two possible situations was most likely (cf. problem-solving tasks), participants had to indicate whether the estimation of the actor was correct (decision-evaluation). The no-conflict version were created by changing the person's or institution's provided unlikely explanation into a likely explanation (cf. problem-solving tasks) and by changing the decision of the new actor into a probability estimation of a conjunctive situation in which an unlikely explanation was added to the likely explanation (e.g., "However, according to an investigative journalist of the Volkskrant, it is more likely that not only the soup contained dangerous additives but that Heinz also wanted to improve the taste of the soup"). In each task, the actor judged the conjunctive situation as more likely than the non-conjunctive situation. Hence, the actor was always incorrect.

Filler tasks. In addition to the 12 problem-solving and 12 vignette tasks, four filler tasks were presented about halfway through (to make the tasks of interest less repetitive and predictable). These were problem-solving tasks in which participants had to find the correct day of the week (cf. Schmeck, Opfermann, Van Gog, Paas, \& Leutner, 2015; Van Gog, Kirschner, Kester, \& Paas, 2012). For example, "Suppose today is Friday. What day is it the day after the day before yesterday?".

Participants completed a total of 28 tasks grouped in five blocks. The first two blocks were always vignette tasks: a block of six base-rate tasks (three conflict, three no-conflict) and a block of six conjunction tasks (three conflict, three no-conflict). The order of these two blocks was randomized and the order of the six tasks within each block was also randomized. Hereafter, participants completed a block with the four filler tasks. The final two blocks were always problem-solving tasks: a block of six base-rate tasks (three conflict, three no-conflict) and a block of six conjunction tasks (three conflict, three no-conflict). Again, the order of the two blocks and of the six tasks within each block was randomized. The vignette tasks were administered first because we were most interested in conflict detection during decision-evaluation on the vignette tasks. Therefore, we wanted to ensure that participants' reasoning evaluation processes were not influenced by prior exposure to problem-solving tasks. We counterbalanced the content of the reasoning tasks across task format and conflict version ${ }^{3}$.

\footnotetext{
${ }^{3}$ Note each task had four versions: a conflict problem-solving version, a no-conflict problem-solving version, a conflict vignette version, and a no-conflict vignette version. Participants completed 24 tasks, hence, there were $24 \times 4=96$ task versions in total.
} 
Response time. On each task, participants' response time was logged from the moment the task was presented on the screen until the participant clicked on one of the two multiple-choice answering options.

Confidence. Immediately after submitting their responses (i.e., their decision on the problem-solving tasks or decision-evaluation on the vignette tasks) participants had to indicate how confident they were that their answer to the reasoning task was correct. Their confidence was measured in percentages from $0 \%$ (not at all confident) to $100 \%$ (completely confidence) that increased in steps of 5\%.

Confidence response time. In line with Frey et al. (2018), we also measured participants' confidence response times. For each confidence rating, we logged the time it took participants to rate their confidence (i.e., the interval between the presentation of the scale and the moment they clicked a percentage point).

\subsubsection{PROCEDURE}

The experiment was run online. All materials were presented in Gorilla software (Anwyl-Irvine et al., 2018). Participants were instructed that the study would take up to 45 minutes and demanded their full attention. After giving informed consent, participants were presented with general instructions on how the experiment should be displayed (full screen and notifications off). Next, an attention check was conducted to see whether the participants had read the full instruction ${ }^{4}$, followed by some demographic questions (age, gender, and educational background). Next, a short reading test was implemented to check for anomalies in reading speed or reading comprehension (adopted from Taalblad.be, Van Kelecom, 2017; none were excluded based on the reading test). To familiarize participants with the confidence measure, they were given three weekday problems (cf. filler tasks) as practice tasks. By varying the problem complexity on these tasks, we also got an indication of whether participants varied their confidence ratings accordingly (which was the case). Then, participants could start with the actual reasoning tasks. After finishing all blocks, one final attention check was administered to determine whether participants still answered the confidence measure attentively. Participants were presented a clearly false statement ("München is the capitol of Germany") and had to indicate whether this statement was correct or incorrect and give their confidence in their answer (96\% answered correctly with an average confidence of $97.6 \%$, $\mathrm{SD}=7.6 ; 4 \%$ answered incorrectly with an average confidence of $45.0 \%, \mathrm{SD}=49.7$ ).

\footnotetext{
${ }^{4}$ The final sentence of the general instruction was: "On the next page you will be asked which button you have to press. Then press space bar." On the next page, a next-button appeared along with the question "Which button do you have to press?". Participants who incorrectly clicked the next-button instead of pressing the spacebar were prompted to the read the general instructions again.
} 


\section{CONFLICT DETECTION IN DECISION-MAKING AND DECISION-EVALUATION}

\subsubsection{DATA ANALYSIS}

All analyses were performed using R version 3.5.2 and run separately for the base-rate and conjunction tasks.

Item-level check. Because the tasks were new, we checked whether the content of the items' cover stories influenced participants' accuracy. We conducted a logistic mixed effects model, with response accuracy (incorrect $=0$; correct $=1$ ) as dependent variable, with item-content number as fixed effect, and with task format (problem-solving $=0$; vignette $=1$ ), conflict version (conflict $=0$; no-conflict $=1$ ), and participants' identification as random effects. Item content did not tend to affect accuracy on the tasks (see Supplementary Material, Section 5.2).

Accuracy. To get an overview of the overall performance, we calculated participants' proportion of correct responses per task format and per conflict version. To test whether accuracy differed between task formats and conflict version, we conducted logistic mixed-effect models with response accuracy as dependent variable (incorrect $=0$; correct $=1$ ), with task format (problem solving $=0$; vignette $=1$ ), conflict version $($ conflict $=0$; no conflict $=1$ ), and the interaction between task format and conflict version as fixed effects, and with item-content number and participants' identification as random effects.

Conflict detection. To provide an overview of the conflict detection indices, we calculated participants' average confidence (\%), response time (s), and confidence response time (s) across their correctly and incorrectly performed trials, per task format and per conflict version. For both task formats, we tested for significant conflict detection effects on all conflict detection indices. For these analyses, we followed the standard practice to only include biased participants, who failed to solve at least one of the conflict tasks correctly (e.g., De Neys et al., 2011; Frey et al., 2018). Per task format, we conducted three linear mixed-effect models with each conflict detection index as dependent variable, with conflict version (conflict $=0$; no conflict $=1$ ) as fixed effect, and with item-content number and participants' identification as random effects ${ }^{5}$. In all analyses using response times, we used log-transformed values. For ease of interpretation we report the raw response time values in the tables and the text.

To see how large the conflict detection effects were, we calculated the difference between participants' confidence ratings or response times on incorrect responses to conflict tasks and on correct responses to no-conflict tasks. The reported group-level conflict detection effect sizes were calculated following a standard procedure (e.g., De Neys et al., 2011; Frey et al., 2018): we subtracted the average confidence/response times on biased participants' correctly solved no-conflict trials from the average confidence/response times on biased participants'

\footnotetext{
${ }^{5}$ For reasons of sample size, we did not test for effects of task format in the main analyses (i.e., then only participants who were biased on both the problem-solving tasks and the vignette tasks could be included). However, we additionally ran these analyses on the smaller sample and report significant effects in the results section.
} 


\section{CHAPTER 5}

incorrectly solved conflict trials. We did not analyze the correctly performed conflict trials, as conflict detection measures on correctly performed conflict trials do not provide a pure indication of conflict detection efficiency per se (De Neys \& Bonnefon, 2013). The few incorrectly answered no-conflict trials were also discarded from further analyses (i.e., it is hard to interpret these trials, since no-conflict trials cue heuristic responses which are congruent with correct performance).

\subsection{RESULTS}

\subsubsection{BASE-RATE TASKS}

Accuracy. Table 5.1 (top panel) presents an overview of participants' average performance on conflict and no-conflict base-rate tasks in problem-solving and vignette format. In both task formats, most participants performed poorly on the conflict tasks. No-conflict tasks, on the other hand, were performed very well. Correct solution rates were comparable to those of previous studies (e.g., Frey et al., 2018 obtained averages of .40 for conflict versions and .85 for no-conflict versions). The logistic mixed-effects model yielded a significant interaction effect between task format and conflict version, $\mathrm{B}=-0.50, \mathrm{SE}=0.24, \mathrm{~W}=-2.05, p=.040$. Follow-up analyses revealed that participants did not differ significantly in response accuracy when solving conflict tasks in problem-solving or vignette format, $\mathrm{B}=-0.20, \mathrm{SE}=0.15, \mathrm{~W}=$ $-1.35, p=.178$, whereas, for no-conflict tasks, participants were significantly more accurate on the problem-solving format compared to the vignette format, $\mathrm{B}=-0.70, \mathrm{SE}=0.20, \mathrm{~W}=$ $-3.46, p<.001$.

Thus, participants showed a similar performance pattern on these base-rate tasks with more realistic scenarios as previously found on classical base-rate problems (i.e., low accuracy on conflict tasks and high accuracy on no-conflict tasks). Furthermore, task format did not affect participants' reasoning accuracy on conflict tasks but it did affect accuracy on no-conflict tasks: participants performed these slightly better in problem-solving format (i.e., decision-making) than in vignette format (i.e., decision-evaluation).

Table 5.1. Average accuracy (SD) on the base-rate and conjunction tasks

\begin{tabular}{lll}
\hline & Conflict & No-conflict \\
\hline Base-rate tasks & & \\
$\quad$ Problem-solving & $.39(.35)$ & $.90(.17)$ \\
$\quad$ Vignette & $.35(.32)$ & $.83(.24)$ \\
Conjunction tasks & & \\
$\quad$ Problem-solving & $.26(.32)$ & $.89(.19)$ \\
$\quad$ Vignette & $.34(.29)$ & $.75(.27)$ \\
\hline
\end{tabular}


Conflict detection. Table 5.2 (top two panels) provides an overview of the average scores on the conflict detection indices for correctly and incorrectly performed trials.

Confidence (\%). Regarding confidence on the problem-solving tasks, we found that biased participants (i.e., participants solving at least one conflict task incorrectly) were significantly less confident about their performance on incorrectly solved conflict tasks than about their performance on correctly solved no-conflict tasks, $\beta=0.26, \mathrm{SE}=0.03, t(585.70)=9.39, p$ $<.001$. Biased participants showed an average confidence decrease of 9.4 percentage points $(\mathrm{SD}=18.6)$. We will refer to this difference as the size of the conflict detection effect (see Data Analysis section). Also for the vignette tasks, we found that participants were significantly less confident on incorrectly evaluated decisions on the conflict tasks than on correctly evaluated decisions on the no-conflict tasks, $\beta=0.25, \mathrm{SE}=0.03, t(575)=8.11, p<.001$, with about a similar conflict detection effect size $(\mathrm{M}=-8.9 \%, \mathrm{SD}=18.4)$ as obtained for the problem-solving task. The additional model testing the effect of task format on the smaller sample (see footnote 5) revealed a significant main effect, $\beta=0.08, \mathrm{SE}=0.03, t(1277.64)=$ $2.40, p=.017$. This indicated that participants were on average more confident about their performance on vignette tasks than about their performance on problem-solving tasks (on both conflict and no-conflict versions). Overall, the conflict detection findings on both task formats were in line with previous studies using classical base-rate problems (e.g., Frey et al., 2018, who found a conflict detection effect with an average size of $-12.3 \%$ ).

Response time (s). Participants' average response time on incorrectly solved conflict problem-solving tasks was not significantly longer than on correctly solved no-conflict problem-solving tasks, $\beta=-0.01, \mathrm{SE}=0.03, t(558.52)=-0.43, p=.670$. Hence, we found no evidence of conflict detection on the response time index, only a very small trend in the descriptive statistics with an average size of $0.7 \mathrm{~s}(\mathrm{SD}=18.6)$. Likewise for the vignette tasks, we did not obtain a significant difference between response time for incorrectly evaluated decisions on conflict tasks and correctly evaluated decisions on no-conflict tasks, $\beta=-0.001$, $\mathrm{SE}=0.03, t(557.82)=-0.05, p=.960$. Average response time for incorrect performance on conflict vignette tasks was even slightly shorter than for correctly performed no-conflict tasks, pointing to a small reversed detection trend in the descriptive statistics $(\mathrm{M}=-0.6 \mathrm{~s}$, $\mathrm{SD}=19.9$ ). Also, the additional model (see footnote 5) revealed a significant main effect of task format, $\beta=0.20, \mathrm{SE}=0.03, t(1260.30)=6.81, p<.001$, indicating that participants took on average longer to complete the vignette tasks than to complete the problem-solving tasks (independent of conflict version). These conflict detection results were not in line with previous findings (e.g., Frey et al. 2018, who did find a conflict detection effect with an average effect size of $1.3 \mathrm{~s}$ ).

Confidence response time (s). Finally, we found a conflict detection effect on the confidence response time index for the problem-solving tasks. Participants took significantly longer to enter their confidence judgements for incorrectly solved conflict tasks than to enter their confidence judgements for correctly solved no-conflict tasks, $\beta=-0.11, \mathrm{SE}=0.03, t(594.44)=$ $-3.80, p<.001$, with an average effect size of $0.3 \mathrm{~s}(\mathrm{SD}=1.3)$. For the vignette tasks, we only found a trend towards conflict detection, $\beta=0.05, \mathrm{SE}=0.03, t(598.20)=-1.65, p=.099$, with a more or less similar average difference $(\mathrm{M}=0.2 \mathrm{~s}, \mathrm{SD}=1.6)$. These findings were party in 


\section{CHAPTER 5}

line with a previous study by Frey et al. (2018), who found a negative trend with an effect size of $-0.3 \mathrm{~s}$.

Thus, in line with previous studies using classical base-rate problems (De Neys et al., 2011; Frey et al., 2018; Thompson \& Johnson, 2014), we found significant conflict detection effects on the confidence index for the current base-rate tasks, both in problem-solving (i.e., decision-making) and in vignette (i.e., decision-evaluation) format. However, in contrast to many previous studies (e.g., Frey et al., 2018; Pennycook, Cheyne, et al., 2014), we found no evidence for conflict detection on the response time index. Finally, for the confidence response time index, we found a conflict detection effect on the problem-solving tasks but not on the vignette tasks. This is partly in line with a previous study by Frey et al. (2018), who found a negative trend.

Table 5.2. Group-level averages (SD) on each of the three conflict detection indices as a function of response accuracy

\begin{tabular}{lcccc}
\hline Conflict detection index & $\begin{array}{c}\text { Conflict: } \\
\text { correct }\end{array}$ & $\begin{array}{c}\text { Conflict: } \\
\text { incorrect }\end{array}$ & $\begin{array}{c}\text { No-conflict: } \\
\text { correct }\end{array}$ & $\begin{array}{c}\text { No-conflict: } \\
\text { incorrect }\end{array}$ \\
\hline Base-rate problem-solving task & $n=104$ & $n=135$ & $n=159$ & $n=43$ \\
$\quad$ Participants by group & $66.7(17.8)$ & $66.8(18.1)$ & $77.1(15.4)$ & $63.5(18.8)$ \\
$\quad$ Average confidence (\%) & $39.9(21.9)$ & $38.3(25.0)$ & $36.5(15.2)$ & $65.6(14.8)$ \\
$\quad$ Average RT (s) & $2.2(1.5)$ & $2.2(1.9)$ & $1.9(1.2)$ & $1.8(1.5)$ \\
$\quad$ Average confidence RT (s) & & & & \\
Base-rate vignette task & $n=102$ & $n=147$ & $n=156$ & $n=64$ \\
$\quad$ Participants by group & $64.6(17.5)$ & $68.9(17.6)$ & $77.7(13.8)$ & $62.9(19.1)$ \\
$\quad$ Average confidence (\%) & $49.8(24.1)$ & $47.2(23.3)$ & $24.2(0.7)$ & $26.2(0.1)$ \\
$\quad$ Average RT (s) & $2.5(1.7)$ & $2.3(1.5)$ & $1.3(0.7)$ & $2.4(2.2)$ \\
$\quad$ Average confidence RT (s) & $n=78$ & $n=145$ & $n=157$ & $n=45$ \\
Conjunction problem-solving task & $n 9.1(16.8)$ & $70.7(16.7)$ & $81.7(14.3)$ & $67.8(17.9)$ \\
$\quad$ Participants by group & $27.4(25.0)$ & $24.0(20.9)$ & $22(16.5)$ & $25.9(16.6)$ \\
$\quad$ Average confidence (\%) & $2.0(1.3)$ & $1.8(0.8)$ & $2.0(1.1)$ & $2.1(1.8)$ \\
$\quad$ Average RT (s) & & & & \\
$\quad$ Average confidence RT (s) & $n=112$ & $n=149$ & $n=155$ & $n=88$ \\
Conjunction vignette task & $58.6(19.4)$ & $65.4(17.0)$ & $71.0(16.8)$ & $65.5(16.8)$ \\
$\quad$ Participants by group & $30.3(16.6)$ & $29.1(15.3)$ & $28.3(17.6)$ & $31.5(19.0)$ \\
$\quad$ Average confidence (\%) & $2.0(1.2)$ & $2.1(1.4)$ & $2.4(2.9)$ & $1.8(1.2)$ \\
$\quad$ Average RT (s) & & &
\end{tabular}

Individual differences. We also explored potential individual differences in conflict detection. Next to investigating whether conflict detection takes place at the averaged group level (i.e., the analyses above), it also relevant to know how many individuals this effect actually applied (for a discussion on this, see Frey et al., 2018). Therefore, we analyzed how many of the biased reasoners showed conflict detection. The interested reader can find a complete 
overview of these results in the Supplementary Materials, Section 5.3. Overall, results showed that the vast majority of the biased reasoners showed conflict detection effects on the conflict detection indices that also yielded significant conflict detection effects at the group level. This was the case for both tasks formats.

Some previous studies found correlations between the conflict detection effect size and performance accuracy on conflict problems (Mevel et al., 2015; Pennycook et al., 2015a). In line with those previous studies, we also calculated correlations between each individual's conflict detection effect size and their total accuracy on the conflict tasks (for a full overview see the Supplementary Materials, Section 5.3). The correlation analyses indicated that for the vignette tasks, conflict detectors with larger confidence and response time effect sizes were more likely to correctly evaluate decisions on conflict tasks (i.e., recognized that a decision was biased). For the problem-solving tasks, on the other hand, we did not find these correlations.

Finally, given the similarity of conflict detection patterns across both task formats, one would expect that individuals who detected conflict on problem-solving tasks, would also detect conflict on vignette tasks. To test this assumption, we used cross-tables and counted how many of the biased participants showed conflict detection across both task formats (see Supplementary Materials, Section 5.3). According to all three conflict detection indices, there was a group of consistent detectors, who showed conflict detection on both the problem-solving tasks and on the vignette tasks, and a relatively small group of consistent non-detectors, who showed no sign of conflict detection in either of the two task formats. Surprisingly, most participants were inconsistent detectors (between $44.0 \%$ and $52.0 \%$ of biased responders), showing conflict detection on only one of the two task formats. For all three conflict detection indices, there were more participants who detected conflict on the problem-solving tasks (i.e., decision-making) than on vignette tasks (i.e., decision-evaluation), although the differences were small.

\subsubsection{CONJUNCTION TASKS}

We conducted the same analyses on the conjunction tasks. In general, the results were consistent with the findings on the base-rate tasks.

Accuracy. Table 5.1 (bottom panel) provides an overview of the average performance on the conjunction problem-solving and vignette tasks. Participants performed poorly on the conflict tasks, whereas they performed well on the no-conflict tasks. Correct solution rates were comparable to those of previous studies (e.g., Frey et al., 2018 obtained an average of .22 for conflict versions and .64 for no-conflict versions). The logistic mixed-effect model yielded a significant interaction effect between task format and conflict version, $\mathrm{B}=-1.49$, $\mathrm{SE}=0.24, \mathrm{~W}=-6.18, p<.001$. Follow-up analyses showed that participants achieved a higher accuracy on conflict tasks in vignette format than on conflict tasks in problem-solving format, $\mathrm{B}=0.44, \mathrm{SE}=0.15, \mathrm{~W}=2.90, p=.003$. For no-conflict tasks, on the other hand, a higher accuracy was achieved on the problem-solving format than on the vignette format, $\mathrm{B}$ $=-1.19, \mathrm{SE}=0.20, \mathrm{~W}=-5.86, p<.001$. 
Thus, participants showed a similar performance pattern on these conjunction tasks with more realistic scenarios as previously found on classical conjunction problems (i.e., low accuracy on conflict tasks and high accuracy on no-conflict tasks). Furthermore, participants reasoned slightly better on conflict tasks in vignette format (i.e., decision-evaluation) but no-conflict tasks were performed better when presented in problem-solving format (i.e., decision-making).

Conflict detection. Table 5.2 (bottom two panels) provides an overview of the average scores on the conflict detection indices for correctly and incorrectly performed trials.

Confidence (\%). The confidence index yielded a significant conflict detection effect on both task formats. Participants showed a decreased confidence on incorrectly solved conflict problem-solving tasks, compared to correctly solved no-conflict problem-solving tasks, $\beta$ $=0.27, \mathrm{SE}=0.02, t(631.94)=11.3, p<.001$. The average conflict detection effect size was $-10.1 \%(\mathrm{SD}=14.3)$. Participants showed the same pattern on the vignette tasks, $\beta=0.13$, SE $=0.03, t(535.63)=4.34, p<.001$, yet with a smaller effect size $(\mathrm{M}=-5.2 \%, \mathrm{SD}=16.7)$. The additional model (see footnote 5) revealed a significant interaction effect between task format and conflict version, $\beta=-0.13, \mathrm{SE}=0.03, \mathrm{t}(1288.58)=-3.81, p<.001$. Hence, this indicated that participants' conflict detection effect size was significantly smaller on vignette tasks. The conflict detection findings were in line with previous studies using conjunction problems (e.g., Frey et al. 2018, who found a conflict detection effect with an average size of $-12.5 \%$ ).

Response time (s). For response time, descriptive statistics seemed to show a trend towards conflict detection with slightly longer response times for incorrect conflict tasks than for correct no-conflict tasks, but this was not significant, on the problem-solving tasks, $\beta=-0.29$, $\mathrm{SE}=0.03, t(626.31)=-1.17, p=.240$ (average difference $1.7 \mathrm{~s} \mathrm{SD}=15.4$ ) or the vignette tasks, $\beta=-0.17, \mathrm{SE}=0.03, t(511.75)=-0.54, p=.590$ (average difference $0.4 \mathrm{~s} \mathrm{SD}=15.8$ ). The additional model (see footnote 5 ) revealed a significant main effect of task format, $\beta$ $=0.24, \mathrm{SE}=0.03, t(1281.58)=8.71, p<.001$, indicating that participants took on average longer to complete the vignette tasks than to complete the problem-solving tasks (both conflict versions). The results on conflict detection were not in line with previous findings (e.g., Frey et al. 2018, who did find a conflict detection effect with an average effect size of $1.2 \mathrm{~s})$.

Confidence response time (s). For the confidence response time index, there seemed to be a trend towards reversed detection in the descriptive statistics, with participants taking slightly longer time to enter their confidence judgements for correctly solved no-conflict tasks than for incorrectly solved conflict tasks. Whereas this was not significant on the problem-solving tasks, $\beta=0.02, \mathrm{SE}=0.03, t(651.81)=0.63, p=.530$ (average difference $-0.1 \mathrm{~s}, \mathrm{SD}=1.0)$ it was significant on the vignette tasks, $\beta=0.08, \mathrm{SE}=0.03, t(545.98)=2.52, p=.012$ (average effect size of $-0.3 \mathrm{~s}, \mathrm{SD}=2.8$ ). These findings were more or less in line with a previous study by Frey et al. (2018), who found a trend towards a reversed conflict detection effect with an average size of $-0.2 \mathrm{~s}$.

In sum, just as with the base-rate tasks, participants showed quite consistent conflict detection patterns across both task formats. As previously found for classical conjunction problems (e.g., De Neys et al., 2011; Frey et al., 2018), participants showed significant 
conflict detection effects on the confidence index on both task formats, although a larger effect was found on the problem-solving tasks. Again, unexpectedly, we found no significant conflict detection effect on the response time index or the confidence response time index; moreover, on the latter, there were indications for a reversed detection effect, which is in line with the negative trend obtained in the study by Frey et al. (2018).

Individual differences. All individual differences analyses are fully reported in the Supplementary Materials, Section 5.3. Overall, results showed that whenever a conflict detection index yielded a significant group level effect, the vast majority showed conflict detection at the individual level too. Interestingly, we found that, within the subgroups of conflict detectors, the confidence conflict detection effect size did not differ between task formats, but, the percentage of conflict detectors did; the percentage of conflict detectors was lower on the vignette format than on the problem-solving format. In addition, in both response formats we found that conflict detectors with larger confidence and response time effect sizes had overall higher accuracies on the conflict tasks. Results were mixed for the confidence response time conflict detection indices. Finally, just as with the base-rate tasks, all three indices indicated that there was a relatively large group of inconsistent conflict detectors (between $43.5 \%$ and $48.8 \%$ of biased reasoners), meaning that they detected conflict either on the problem-solving tasks or on the vignette tasks. Again, all three indices suggested that slightly more participants detected conflict in the problem-solving context than in decision-evaluation context.

\subsection{DISCUSSION}

Thus far, research on conflict detection has studied this effect only during reasoning on classical, arguably non-realistic, heuristics-and-biases tasks. The present study investigated conflict detection with more relevant "real-world" scenarios and aimed to establish whether people would also detect biased reasoning in decisions of others, and if not, whether they at least show signs of conflict detection.

\subsubsection{ACCURACY}

In line with previously studied classical heuristics-and-biases tasks (e.g., Frey et al., 2018; Pennycook et al., 2015a; Raoelison \& De Neys, 2019; Thompson et al., 2011), participants performed very well on the no-conflict versions of our tasks with more realistic scenarios and performed quite poorly on the conflict versions. This applied to both task types (base-rate and conjunction) and both task formats (problem-solving and vignette). Yet, there was an additional effect of task format on reasoning accuracy. No-conflict tasks were performed better when presented in problem-solving (decision-making) format than in vignette (decision-evaluation) format; this applied to both task types. Conflict tasks were performed equally well in both task formats of the base-rate tasks, but, for the conjunction tasks, we found that conflict tasks were performed slightly better when presented in vignette format. The higher performance on the no-conflict problem-solving tasks may simply indicate that 
participants improved due to the repeated task presentation, as the problem-solving tasks were always performed after the vignette tasks. Given that the vignette tasks were always completed before the problem-solving tasks, it is unlikely that the better performance on these tasks results from a general repeated task presentation effect. Hence, this could indicate that, for the conjunction tasks, participants were better at recognizing someone else's biased decision (vignette tasks) than at making an unbiased decision themselves (problem-solving tasks). This would align with the suggestion that people become more deliberative and critical when they have to judge the argumentation of others than when they themselves have to make a judgment (Mercier \& Sperber, 2011; Trouche et al., 2016). Note, however, that the average performance difference between the two task formats was not that large (i.e., 12\%).

\subsubsection{CONFLICT DETECTION}

With regard to conflict detection, the confidence index showed clear and consistent conflict detection effects, yet, the response time and confidence response time indices yielded inconsistent results across task types and formats. For the confidence index, we found significant conflict detection effects on both base-rate and conjunction tasks in both problem-solving and vignette format. In line with many previous studies (Bago \& De Neys, 2017; De Neys et al., 2011, 2013; De Neys \& Feremans, 2013; Gangemi et al., 2015; Thompson \& Johnson, 2014), participants were, on average, less confident about their incorrect performances on conflict tasks than about their correct performances on no-conflict tasks. For the conjunction tasks, the results additionally showed that the conflict detection effect size on the vignette task format was significantly smaller than on the problem-solving task format. Interestingly, further individual differences analyses revealed that it was not so much the size of the conflict detection effect, but the total percentage of biased participants showing conflict detection, that differed across tasks formats. That is, the confidence effect size for both subgroups of conflict detectors (only) was quite similar, but the percentage of conflict detectors on vignette tasks was smaller than on problem-solving tasks. In other words, fewer participants seemed aware of their errors when evaluating decisions of others (i.e., vignette tasks) than when making decisions themselves (i.e., problem-solving tasks). It could be that - in addition to a group of participants who become more deliberate when evaluating other people's decisions (cf. accuracy results) - there is also group of reasoners who are less motivated to pay attention to other people's decisions when these do not directly affect them and are in line with their intuitive ideas.

The response time and confidence response time indices did not appear to be reliable measures of conflict detection on the current tasks. We found no significant conflict detection effects on the response time index, and effects on the confidence response time index were inconsistent across task types and formats. The lack of response time effects is in stark contrast with many previous studies (Bonner \& Newell, 2010; De Neys \& Glumicic, 2008; Pennycook, Trippas, et al., 2014; Stupple \& Ball, 2008). The most likely explanation for this seems to lie in the more realistic scenarios used in our tasks, which made them more lengthy than classical heuristics-and-biases tasks. In comparison with classical tasks, 
average response times on the current tasks were very long and the variances were rather large. Hence, subtle differences in task processing could probably not reliably be captured with such response times. A potential solution might be to design shorter tasks or to apply a rapid-response paradigm in which the descriptive information is presented serially to obtain less noisy reasoning time measures (cf. Pennycook et al., 2014). Note, however, that both solutions would render the tasks less similar to real-world reasoning situations, which was the goal here. The inconsistent confidence response time results were in line with findings of Frey et al. (2018) and endorse their suggestion that this index may not be a reliable measure of conflict detection (see also Šrol \& De Neys, 2019). Thus, future studies that consider conflict detection with longer or more complex tasks can best rely on confidence measures or investigate other potential measures of conflict detection (e.g., reasoning effort, or process measures obtained through eye-tracking).

\subsubsection{CONSISTENCY IN CONFLICT DETECTION}

Taken together, the current results (especially on the confidence measures) suggest that conflict detection also occurs during reasoning on longer, more complex, and realistic reasoning tasks than studied before. In addition, the results indicated that the conflict detection effect was very similar on problem-solving and vignette tasks, except for the finding that somewhat fewer participants were conflict detectors on the conjunction vignette tasks. Yet, the individual differences results related to also pointed to a potential effect of task format. These results showed that nearly half of the biased reasoners was a consistent detector (i.e., detected conflict in both task formats), yet, an equally large part was inconsistent detector (i.e., detected conflict in only one of the two task formats). Also, all three conflict detection indices (on both conjunction and base-rate tasks) indicated that more participants detected conflict on the problem-solving tasks than on the vignette tasks. Although the differences were small, these results could imply that conflict detection is fairly task-format or domain-specific (cf. Frey \& De Neys, 2017; Šrol \& De Neys, 2019) and that it was slightly more challenging to detect conflict in vignette tasks. In other words, detecting conflict in task A does not necessarily imply that you will also detect it in in task B.

\subsubsection{LIMITATIONS}

This study took a first step towards investigating conflict detection in more realistic scenarios and in evaluating other people's decisions. However, the current tasks are not yet comparable to real-world reasoning. That is, for experimental rigor, all tasks were similarly structured, well-structured, included all necessary information to make an adequate probability estimation. In addition, on the vignette tasks, participants' attention was always directed explicitly to the relevant reasoning part (i.e., they were explicitly asked whether a specific estimation in the text was correct or not). Our findings suggest, though, that it would be fruitful for future research to start addressing conflict detection indices in (gradually) more realistic reasoning contexts (e.g., evaluating decisions of two people engaged in a dialogue). 


\section{CHAPTER 5}

\subsubsection{CONCLUSION}

In conclusion, the present study suggests that conflict detection also occurs on longer, more complex, and more realistic reasoning tasks than the classical heuristic-and-biases tasks studied before. Moreover, conflict detection occurs not only when making a decision oneself, but also when evaluating decisions of others (as described in a text). This is relevant because there are many everyday situations in which we are confronted with (biased) conclusions or decisions made by others and have to evaluate or form our own opinion on those decisions. Hence, these findings improve our understanding of reasoning in everyday scenarios, showing that even if people may not detect biased reasoning in decisions of others, they often do show signs of conflict detection. The current findings are especially relevant for (studying) reasoning in contexts in which recognizing errors is important; for instance, in medicine, where doctors often have to evaluate initial diagnoses of others, or in education, where teachers have to detect and give feedback on biases in their students' reasoning. Even though people may err when evaluating other's reasoning, there seems to be some error or conflict detection going on. One may envisage how future training could try to build on the currently demonstrated error signal to de-bias people's evaluative reasoning. 
CONFLICT DETECTION IN DECISION-MAKING AND DECISION-EVALUATION 


\section{Chapter 6}

\section{"You're wrong!": The impact of accuracy}

\section{feedback on the bat-and-ball problem}

This chapter was published as:

Janssen, E. M., Raoelison, M., and De Neys, W. (2020), "You're wrong!" : The impact of accuracy feedback on the bat-and-ball problem. Acta Psychologica, 206, 103042. doi:10.1016/j.actpsy.2020.103042

\section{Acknowledgement of author contributions:}

EJ, MR, and WdN designed the study, EM and MR recruited participants and collected the data, EJ and MR analyzed the data, EJ drafted the manuscript, all authors contributed to critical revision of the manuscript, WdN supervised the study. Additional note: Tim Mainhard checked the data package. 


\section{ABSTRACT}

The popular bat-and-ball problem is a relatively simple math riddle on which people are easily biased by intuitive or heuristic thinking. In two studies we tested the impact of a simple but somewhat neglected manipulation - the impact of minimal accuracy feedback - on bat-and-ball performance. Participants solved a total of 15 standard and 15 control versions of the bat-and-ball problem in three consecutive blocks. Half of the participants received accuracy feedback in the intermediate block. Results of both studies indicated that the feedback had, on average, no significant effect on bat-and-ball accuracy over and above mere repeated presentation. We did observe a consistent improvement for a small number of individual participants. Explorative analyses indicated that this improved group showed a more pronounced conflict detection effect (i.e., latency increase) at the pretest and took more deliberation time after receiving the negative feedback compared to the unimproved group.

Keywords. reasoning; heuristics and biases; bat-and-ball problem; feedback; learning; conflict detection. 


\section{CHAPTER 6}

\subsection{INTRODUCTION}

Although humans have unique cognitive abilities to reason, human reasoning can also be biased. For instance, investors can make bad investment decisions based on the mere familiarity of a stock (Oster \& Koesterich, 2013), doctors can make diagnostic errors due to patients' disruptive behaviors (Schmidt et al., 2016), or judges can misinterpret evidence because of intuitive stereotypical associations (Eberhardt et al., 2006; Thompson \& Schumann, 1987). Reasoning and decision-making studies often attribute this bias to the human tendency to base judgments on fast intuitive impressions rather than on more deliberate reasoning (e.g., Evans, 2008; Kahneman, 2011; Stanovich \& West, 2000; Thompson et al., 2011). This intuitive or so-called "heuristic" thinking can be useful because it is fast and effortless and frequently cues good decisions. However, the problem is that heuristics can also cue decisions that conflict with more logical considerations. In this case, following the intuitive heuristic will lead you astray.

Researchers have been studying heuristic bias empirically with reasoning problems in which an intuitively cued heuristic response conflicts with elementary logical principles. One task that has been studied extensively in the reasoning and decision literature is the bat-and-ball problem in Frederick's (2005) Cognitive Reflection Test (CRT), which states:

A bat and a ball together cost $\$ 1.10$. The bat costs $\$ 1$ more than the ball.

How much does the ball cost?

Most reasoners intuitively conclude that the ball must cost 10 cents $(\$ 1+\$ 0.10=\$ 1.10)$. However, this conclusion is incorrect because in this scenario the bat costs 90 cents more than the ball instead of $\$ 1$. After some reflection, it should become clear that the correct answer requires a different calculation leading to the conclusion that the ball costs 5 cents $(\$ 1.05+\$ 0.05=\$ 1.10)^{1}$. Although the correct "5 cents" answer does not require strong mathematical skills, numerous studies have shown that even educated reasoners fail to solve the problem correctly (Frederick, 2005; Toplak et al. 2014a; Travers et al., 2016), even after repeated problem presentation (Meyer et al., 2018; Raoelison \& De Neys, 2019; Stagnaro et al., 2018). Correct solution rates among university students are typically very low (about $32 \%$; for a meta-analysis, see Brañas-Garza et al., 2019).

The key goal of the present study was straightforward. We wanted to look at the impact of a simple but somewhat neglected manipulation - the impact of minimal response feedback (i.e., telling participants whether their response is correct or incorrect) - on people's bat-and-ball performance. With minimal response feedback we refer to a simple correct/incorrect

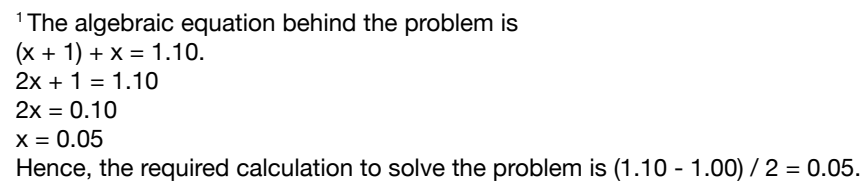


assessment that is presented to the reasoner after (s)he has answered a problem. Although there are some isolated exceptions (e.g., Ball, 2013; Zizzo et al., 2000) such feedback is usually not presented in research on reasoning and heuristic bias (Ball, 2013; Evans, 2002; Kahneman, 2011). Hence, participants are typically not told whether their response is correct or not. In other fields - such as perception and memory research - presenting performance feedback is a common procedure and has sometimes been shown to boost performance (e.g., Ball et al., 2010; Chun \& Wolfe, 1996; Donnelly et al., 2007; Hays et al., 2010). Therefore, it might be worthwhile to examine its impact on heuristic bias as well.

In addition to examining whether accuracy feedback can help to improve reasoning performance, it is also relevant to explore how it works. To gain more insight in the nature of the potential effect of feedback, we measured its effects using a two-response paradigm. In the two-response paradigm reasoners first have to enter their initial, intuitive response to a problem and, thereafter, they can take as much time as they need to deliberate and reflect on their final response (Thompson, Prowse Turner, \& Pennycook, 2011). To make maximally sure that people do not deliberate during the initial response generation, they have to provide it under time pressure, while - at the same time - their working memory is loaded with a memorization task (Bago \& De Neys, 2017, 2019; De Neys, 2017; Newman et al., 2017; Raoelison \& De Neys, 2019). Hence, the procedure deprives participants of the resources they need to efficiently deliberate in the initial response stage (Bago \& De Neys, 2019). In the current study, we used the two-response paradigm to see whether feedback affected people's intuitive and deliberate reasoning differently. For example, a feedback effect on both initial and final responses would indicate that reasoners can automatize the correct reasoning, whereas an effect on merely final responses would imply that reasoners have to keep correcting their intuitive responses to be able to reason correctly.

We conducted two studies in which we explored the impact of feedback on bat-and-ball reasoning while applying the two-response paradigm. Participants solved three consecutive blocks of problems. Half of the participants received accuracy feedback in the intermediate block. In Study 1, we tested the potential impact of feedback with a multiple-choice response format and a quasi-experimental design. That is, we compared data from a previous study (Raoelison \& De Neys, 2019) where participants solved bat-and-ball problems without receiving feedback (no-feedback condition) with new data specifically collected for the purpose of this study, in which bat-and-ball reasoners received the feedback manipulation in the intermediate block (feedback condition). With Study 2, we aimed to replicate the results of Study 1, while applying a full experimental design with a free-response format.

In addition to response accuracy, we also logged participants' response times for explorative analyses. First, we wanted to explore whether the provided feedback affected participants' response latencies. Second, we wanted to use the response latencies as a proxy of conflict or error detection. That is, we were interested in whether biased reasoners' showed some sensitivity to the fact that their heuristic response conflicted with the correct response. Such conflict or error detection has been shown to result in an increase in response latencies (when contrasted with latencies for easy control problems in which the heuristic response is also correct, see e.g., Bago \& De Neys, 2017, 2019; De Neys, 2012; Frey et al., 2018; 
Johnson et al., 2016; Pennycook et al., 2015a). We wanted to explore whether individuals whose accuracy improved after feedback were characterized by differential conflict detection effects.

\subsection{STUDY 1}

\subsubsection{METHOD}

Participants. A total of 50 participants were recruited for the feedback condition (all gave informed consent). Half of the participants $(n=25)$ were volunteers, recruited via personal networks. The other half $(n=25)$ was recruited on Prolific Academic (www.prolific.ac) and were paid at a rate of $£ 5 / \mathrm{h}$. All participants were native English speakers (30 females; age: M $=27.4$ years, $\mathrm{SD}=8.5)^{2}$. Most participants reported high school $(50.0 \%)$ or a Bachelor degree $(38.0 \%)$ as highest completed level of education, followed by a Master's degree $(6.0 \%)$, less than high school $(2.0 \%)$ and a Doctoral degree $(2.0 \%)$, respectively.

We used data of a previous a study (Raoelison \& De Neys, 2019) as a base-line to compare with our feedback data. In this study, 62 participants solved the same bat-and-ball problems without receiving feedback (no-feedback condition). All participants in this study were also native English speakers and recruited on Prolific Academic and were also paid at a rate of $£ 5 / \mathrm{h}$ (38 females; age: $\mathrm{M}=35.5$ years, $\mathrm{SD}=13.2$ ). Most participants reported a Bachelor degree $(46.8 \%)$ or high school $(35.5 \%)$ as highest completed level of education, followed by a Master's degree (12.9\%), less than high school (3.2\%), and a Doctoral degree $(1.6 \%)$, respectively.

Materials. The materials for the feedback condition were taken from the study by Raoelison and De Neys (2019), who designed a total of 110 items to test the robustness of biased responding on bat-and-ball problems by examining how responding is affected by repeated problem presentation. We used 33 items out of their 110 items. Of those 33 items, 15 items were variations of the bat-and-ball problem that had the same underlying structure as the original problem but different superficial item content (e.g., "In a company there are 150 men and women in total. There are 100 more men than women. How many women are there?"). Each problem specified two types of objects with different quantities instead of prices ( e.g., see Bago \& De Neys, 2019; Mata et al., 2017). Each of the 15 problems featured unique content with a total amount that was a multiple of ten and ranged from 110 to 650 (Raoelison $\&$ De Neys, 2019). Each problem was presented with four answer options; the correct response (" 5 cents" in the original bat-and-ball), the intuitively cued "heuristic" response ("10 cents" in the original bat-and-ball), and two foil options. Mathematically speaking, the correct equation to solve the standard bat-and-ball problem is: $\$ 1.00+2 \mathrm{x}=\$ 1.10$ (see footnote 1 ), instead, people are thought to be intuitively using the " $\$ 1.00+x=\$ 1.10$ " equation to determine their response (Kahneman, 2011). We always used the latter equation to determine

\footnotetext{
${ }^{2}$ Demographic information of one participant is missing because he/she dropped out before completing the demographic questions at the end.
} 
the "heuristic" answer option, and the former to determine the correct answer option for each problem. Following Bago and De Neys (2019), the two foil options were always the sum of the correct and heuristic answer (e.g., "15 cents" in original bat-and-ball units) and their second greatest common divider (e.g., "1 cent" in original units). For each item, the four response options appeared in a randomly determined order. The following illustrates the full item format:

In a company there are 150 men and women in total.

There are 100 more men than women.

How many women are there?

$\begin{array}{ll}\text { o } & 50 \\ \text { o } & 75 \\ \text { o } & 5 \\ \text { o } & 25\end{array}$

One possible cause for a lack of an intervention effect is that participants simply become bored with the repeated problem presentation and stop paying attention. To avoid that the task would become too repetitive and to verify that participants stayed minimally engaged in the task there were also 15 control problems. In the standard bat-and-ball versions the intuitively cued "heuristic" response cues an answer that conflicts with the correct answer, hereafter referred to as "conflict" problems. In the "no-conflict" control problems, the heuristic intuition was made to cue the correct response option. This was achieved by deleting the critical relational "more than" statement (De Neys et al., 2013; Travers et al., 2016). With the above example, a control problem version would look as follows:

In a company there are 150 men and women in total.

There are 100 men.

How many women are there in the company?

$\begin{array}{ll}\text { o } & 50 \\ \text { o } & 75 \\ \text { o } & 5 \\ \text { o } & 25\end{array}$

In this case the intuitively cued "50" answer was also correct. We presented the same four answer options as for a corresponding standard conflict version. We added three words to the control problem question (e.g., "in the company") so that standard "conflict" and control "no-conflict" versions had roughly the same length. Given that the control items can be solved correctly on the basis of mere intuitive reasoning, we expected to see ceiled performance on the control items throughout, if participants are paying minimal attention to the task and refrain from mere random responding. 
Finally, in addition to the 15 conflict and the 15 no-conflict problems, there were also 3 filler problems in which participants simply had to add two quantities. For example,

A tech company is offering 100 Motorola phones and 10 Samsung phones.

How many phones are they offering in total?

$\begin{array}{ll}\text { o } & 110 \\ \text { o } & 90 \\ \text { o } & 250 \\ \text { o } & 1000\end{array}$

The rationale behind the filler problems was that these would further help to render the task less repetitive and predictable. In total, participants had to solve 33 problems. The problems were grouped into three blocks (i.e., pretest, intermediate, and posttest) containing each 5 standard conflict problems, 5 control no-conflict problems, and one filler problem. The filler problem was always presented as the sixth problem in a block. Conflict and no-conflict problems were presented in a randomized order. Participants could take a short break after completing each block. We logged both response accuracy and response times on all 33 problems.

As noted, data from the original Raoelison and De Neys (2019), was used as no-feedback baseline. Blocks were similarly structured as the blocks in the feedback condition. In the Raoelison and De Neys study, participants solved a total of 10 blocks. Only the data from the first 3 blocks were analyzed in the current study ${ }^{3}$.

Procedure. The experiment was run online on the Qualtrics platform. Participants were specifically instructed that the experiment demanded their full attention throughout. As in Raoelison \& De Neys (2019), the study adopted the two-response procedure from Bago and De Neys $(2017,2019)$. Participants were instructed they had to provide two consecutive responses for each problem. They were told that we were interested in their very first, initial answer that came to mind and that - after selecting their initial response - they could reflect on the problem and take as much time as they needed to provide a final answer. To minimize the possibility that participants deliberated during the initial response stage, the initial response had to be generated within a stringent response deadline and while cognitive resources were burdened with a secondary load task. The deadline for the initial response was set to $5 \mathrm{~s}$, based on the pretesting of Bago and De Neys (2019) who established that this amounted to the time needed to read the problem. The load task was based on the dot memorization task (Miyake et al., 2001). Before each reasoning problem, participants were presented with a complex visual pattern (i.e., 4 crosses in a $3 \times 3$ grid) they had to memorize while solving the reasoning problem. After answering the reasoning problem the first time (intuitively), participants were shown four different matrices and had to choose the correct,

\footnotetext{
${ }^{3}$ Because the 10 blocks in Raoelison and De Neys (2019) were presented in a randomized order and the current study only used participants' first 3 blocks, the exact superficial item content was a random selection of the 110 items in the 10 blocks and hence differed in both conditions.
} 
to-be-memorized pattern (see Raoelison \& De Neys, 2019). The load and deadline were applied only during the initial response stage and not during the subsequent final response stage in which participants were allowed to deliberate.

Every trial started with a fixation cross shown for $2000 \mathrm{~ms}$. We then presented the first sentence of the problem (e.g., "In a company there are 150 men and women in total.") for $2000 \mathrm{~ms}$. Next, the target pattern for the memorization task was presented for $2000 \mathrm{~ms}$. Afterwards the full problem was presented. At this point, participants had $5000 \mathrm{~ms}$ to give an answer; after $4000 \mathrm{~ms}$ the background of the screen turned yellow to warn participants about the upcoming deadline. If they did not provide an answer before the deadline, they were asked to pay attention to provide an answer within the deadline on subsequent trials. After the initial response was entered, participants were presented with four matrix patterns from which they had to choose the correct, to-be-memorized pattern. Once they provided their memorization answer, they received feedback as to whether it was correct. If the answer was not correct, they were also asked to pay more attention to memorizing the correct pattern on subsequent trials.

Finally, the same item was presented again, and participants were asked to provide a final response. The presentation order of the response options was always the same in the initial and final response stage but was randomized across trials. Once participants clicked on one of the answer options they were automatically advanced to the next trial. The color of the answer options was green during the first response, and blue during the final response phase, to visually remind participants of which question they were answering. Therefore, right under the question we also presented a reminder sentence: "Please indicate your very first, intuitive answer." and "Please give your final answer.", respectively, which was also colored as the answer options. At the very end of the experiment, participants were shown the standard bat-and-ball problem and were asked whether they had seen it before. We also asked them to enter the solution. Finally, participants completed a page with demographic questions.

Feedback manipulation. In both the feedback and no-feedback condition participants were informed when they had completed a block (i.e., after 11 trials), how many blocks were still left and instructed to press the 'Next' button when they were ready to continue with the next block. Participants in the feedback condition additionally received the feedback manipulation in the second (intermediate) block. After completing the pretest block, they received the standard information message that they had completed a block and were additionally informed that they would receive feedback about their performance at the intuitive stage in the next block. The feedback was given immediately after participants had entered their intuitive response. The feedback said either "Correct!" or "Incorrect!". Participants then had to click on 'Next' to complete the load task and to give their final response. The feedback was given after all 11 problems during this block.

Exclusion criteria. We analyzed all conflict and no-conflict trials, which were 30 trials $\times 112$ participants $=3360$ trials in total. Participants failed to provide their first answer before the deadline on 113 trials (3.4\% of all trials) and further failed to pick the correct matrix for the load task on 353 trials ( $10.9 \%$ of remaining trials). Since we could not guarantee that the initial 
response for these trials did not involve any deliberation, we discarded them and analyzed the 2894 remaining trials $(86.1 \%$ of all trials). On average each participant contributed 12.9 $(\mathrm{SD}=2.0)$ conflict trials and $13.0(\mathrm{SD}=2.1)$ no-conflict trials. Note that following Raoelison \& De Neys (2019) we did not exclude participants based on their response to the familiarity question $^{4}$.

\subsubsection{RESULTS AND DISCUSSION}

Response accuracy. For each participant, we calculated the average proportion of correct initial and final responses on the conflict problems and no-conflict problems in each of the three blocks (pretest, intermediate, posttest). Figure 6.1 (top panel) provides an overview of the average performance on the conflict problems of the feedback and the no-feedback condition. We first focus on the response accuracies for the final responses. Figure 6.1 shows that most participants failed to solve the conflict problems correctly in the first block (pretest). On average, the final response accuracy at the pretest was $28.2 \%(\mathrm{SE}=6.0)$ for the feedback condition and $27.7 \%(\mathrm{SE}=5.4)$ for the no-feedback condition. Both conditions improved in average performance from pretest to posttest, but the feedback condition improved, with an average increase of $14.9 \%(\mathrm{SE}=4.5)$, more than the no-feedback condition, which had an average increase of $9.4 \%$ ( $\mathrm{SE}=3.0)$. An ANOVA on final accuracy with block (pretest vs. posttest) as within-subjects factor and condition (feedback vs. no feedback) as between-subjects factor revealed a main effect of block, $F(1,110)=20.77, p<.001, \eta_{\mathrm{p}}^{2}=$ .159 . There was, however, no main effect of condition, $F(1,110)=0.16, p=.688, \eta_{\mathrm{p}}^{2}=.014$, neither an interaction of block with condition, $F(1,110)=1.12, p=.293, \eta_{p}^{2}=.010$. Thus, reasoners in both conditions improved their final accuracy on the conflict reasoning problems over time, but - despite a small observed trend towards a better improvement for the feedback condition - there was no effect of feedback on participants' reasoning performance.

We repeated all the analyses on the final accuracies with the initial accuracies. Except for slightly lower performance averages, the results were fully consistent (see also Figure 6.1). On average, the initial response accuracy at the pretest was $18.6 \%(\mathrm{SE}=4.4)$ for the feedback condition and $23.0 \%(\mathrm{SE}=4.4)$ for the no-feedback condition. Both conditions improved in average performance from pretest to posttest, but the feedback condition improved, with an average increase of $23.0 \%(\mathrm{SE}=5.4)$, more than the no-feedback condition, which had an average increase of $11.7 \%(\mathrm{SE}=3.5)$. Again, the ANOVA showed that reasoners in both conditions improved their accuracy from pretest to posttest but were not affected by the feedback manipulation, block: $F(1,110)=30.60, p<.001, \eta_{\mathrm{p}}^{2}=.218$, condition: $F(1,110)=$ $05, p=.828, \eta_{\mathrm{p}}^{2}=.002$; block $\times$ condition interaction: $F(1,110)=3.68, p=.058, \eta_{\mathrm{p}}^{2}=.032$.

${ }^{4}$ Exploratory analyses confirmed that the pattern of results was similar when this exclusion criterion was applied or not. Reported results concern the full dataset. 


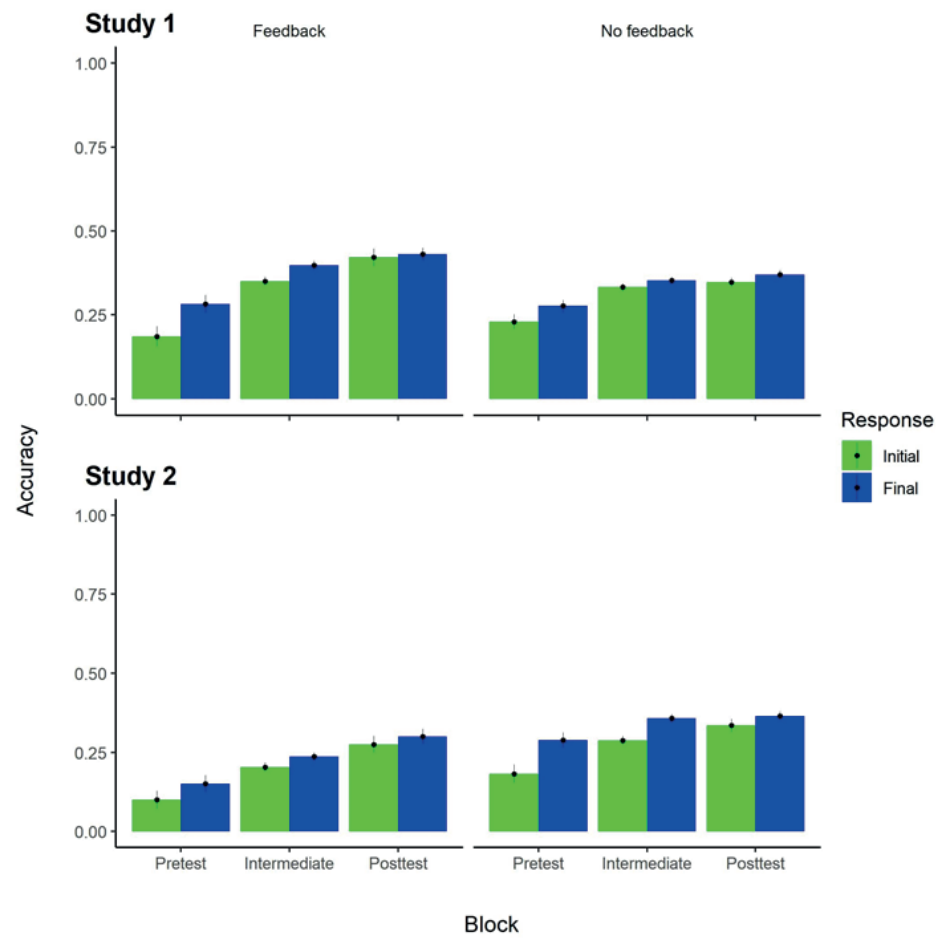

Figure 6.1. Average initial and final accuracy on conflict problems. Error bars are standard errors.

As expected, for the no-conflict control problems we observed a performance at ceiling for both conditions in all blocks with grand means of $95.8 \%(\mathrm{SE}=0.7)$ and $99.0 \%(\mathrm{SE}=0.3)$ for initial and final accuracy, respectively (see also Figure S6.1 in the Supplementary Material). An ANOVA on initial accuracy showed that there was no main effect of block, $F(1,110)=$ $3.35, p=.070, \eta_{\mathrm{p}}^{2}=.030$, no effect of condition, $F(1,110)=0.65, p=.422, \eta_{\mathrm{p}}^{2}=.006$, or an interaction, $F(1,110)=0.76, p=.386, \eta_{p}^{2}=.007$. For final accuracies none of the factors reached significance, block: $\mathrm{F}(1,110)=0.96, p=.331, \eta_{\mathrm{p}}^{2}=.009$; condition: $F(1,110)=0.21$, $p=.646, \eta_{\mathrm{p}}^{2}=.002$; block $\times$ condition: $F(1,110)=0.46, p=.833, \eta_{\mathrm{p}}^{2}<.001$.

In sum, as observed by Raoelison and De Neys (2019), mere repeated problem presentation resulted in a slight performance increase on standard bat-and-ball problems. However, providing feedback did not result in a further significant improvement per $\mathrm{se}^{5}$.

\footnotetext{
${ }^{5}$ To be absolutely sure that the feedback had no effect, we conducted some additional explorative analyses: (1) we conducted mixed effect logistic regression analyses on the dichotomous item accuracy responses with subjects' ID as random effect, yielding comparable results (see Table S6.1 in the Supplementary Material); (2) we controlled for effects of sex as covariate or moderator (Bosch-Domènech et al., 2014; Brañas-Garza et al., 2019), also yielding similar results (see Tables S6.2-S6.4 in the Supplementary Materials); and (3) we repeated all the analyses on a subsample in which we only included the participants that failed to solve any of the pretest conflict problems (i.e., the participants that already solved pretest conflict problems correctly could not further benefit from the feedback), again yielding similar results.
} 


\section{CHAPTER 6}

Individual level directions of change. To gain more insight into how participants solved the problems, we additionally performed a direction of change analysis (Bago \& De Neys, 2017, 2019) for each individual participant. More specifically, on each trial people can give a correct or incorrect response in each of the two response stages. Consequently, this can result in four different types of answer patterns on any single trial (" 00 ", incorrect response in both stages; "11", correct response in both stages; "01", initial incorrect and final correct response; "10", initial correct and final incorrect response). Figure 6.2 (top panel) plots the direction of change classification on each of the consecutive 15 conflict problems ( 5 per block) for each individual participant.

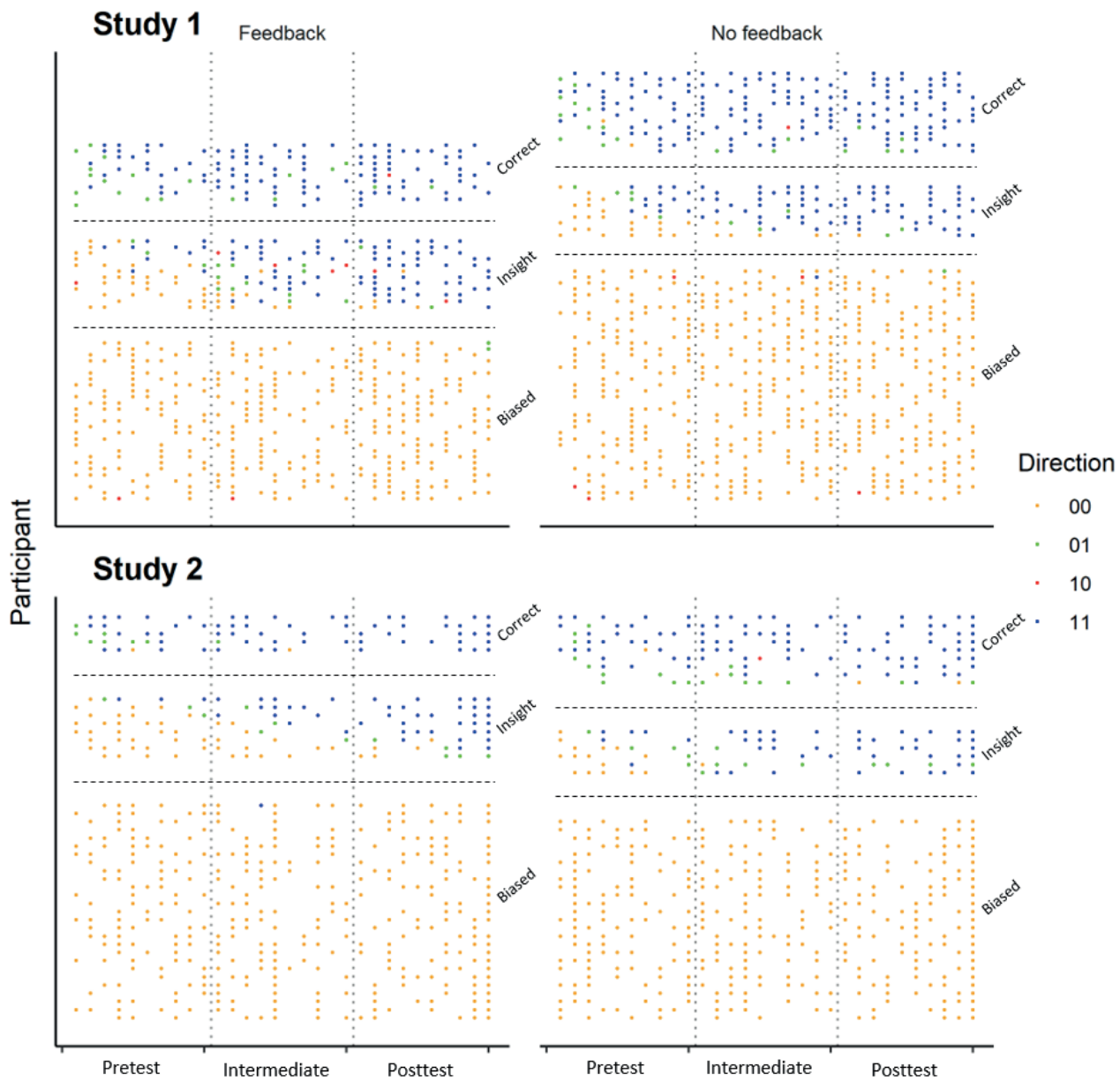

Figure 6.2. Individual trajectories (each row represents one participant). Due to discarding of missed deadline and load trials (see Exclusion criteria), not all participants contributed 15 analyzable trials. Participants are ranked based on the sum of their total initial and final response accuracy. 
As the figure indicates, the overall pattern was very similar in the feedback and no-feedback condition. We first describe the main trends applying to both conditions and end with a comparison between them. By and large, as in Raoelison and De Neys (2019), we can classify the participants in three main groups. First, most participants (66 out of 112 participants or $58.9 \%$ ) predominantly gave 00 responses from start to finish. Hence, the majority of the participants consistently gave incorrect intuitive and deliberate responses and remained biased throughout the study. This group is labeled as the "biased" group in Figure 6.2 . Second, $22.3 \%$ of the participants (25 out of 112) already gave a correct (final) response at their very first trial and predominantly remained responding correctly throughout the study. This group of "correct" reasoners obviously did not need any intervention to arrive at the correct answer. Third, 18.8\% (21 out of 112) started with an incorrect (final) response and found the correct answer somewhere along the way. Once they had found the solution, they remained correct on almost all subsequent trials. We labeled this group as the "insight" group. Interestingly, both in the "correct" and "insight" group we see that for most reasoners, correct responding initially occurs during the deliberation (final response) stage (i.e., a " 01 " response). However, after only one or two trials, they also managed to solve the subsequent problems correctly without deliberation (i.e., already correct at the intuitive response stage, i.e., a "11" response). This suggests that most participants who found the correct answer automatized the reasoning very quickly.

We now turn to the comparison between conditions. The key question is whether it was more likely to gain "insight" after receiving accuracy feedback. Hence, we simply tallied how many individuals in the insight group started responding correctly after the onset of feedback (i.e., after the pretest). This was the case for 6 participants $(12.0 \%$ of total $n=50)$. For comparison, in the no-feedback condition there were 3 participants $(4.8 \%$ of total $n=$ 62) who showed the insight pattern after the pretest block. Hence, these effects put the group level effects further in perspective. There is some evidence for a small intervention trend but this trend is driven by only a handful of participants. The vast majority of biased reasoners' is completely unaffected by feedback (or mere repeated presentation in the no-feedback condition).

Response latencies. We additionally explored whether feedback affected participants' response latencies. For each participant, we calculated the average final response time ${ }^{6}$ on the conflict problems and on the no-conflict problems in each of the three blocks, while distinguishing between correct and incorrect final responses. To reduce the impact of outliers, we used log-transformations which were then back transformed to enhance the interpretation. Furthermore, we excluded all trials with final response times $>120$ s (i.e., $>10.5$ SDs above average). This concerned two conflict trials and one no-conflict trial. Given the explorative nature, we only report descriptive trends.

Figure 6.3 (top panel) provides an overview of the average final response times of the feedback and the no-feedback condition on the conflict problems (see Figure S6.2 in the Supplementary Material for an overview of response time on the no-conflict problems).

${ }^{6}$ Given that initial responses were given under fixed response deadline we focus exclusively on the unrestricted final response times. 


\section{CHAPTER 6}

In the no-feedback condition we observed a general speeding-up after the pretest block, both for correct responses (pretest: $\mathrm{M}=7.75 \mathrm{~s}, \mathrm{SE}=1.19$; intermediate: $\mathrm{M}=4.01 \mathrm{~s}, \mathrm{SE}$ $=0.51$; posttest: $\mathrm{M}=4.54 \mathrm{~s}, \mathrm{SE}=1.19$ ) and incorrect responses (pretest: $\mathrm{M}=4.06 \mathrm{~s}, \mathrm{SE}=$ 0.50 ; intermediate: $\mathrm{M}=3.04 \mathrm{~s}, \mathrm{SE}=0.32$; posttest: $\mathrm{M}=3.93 \mathrm{~s}, \mathrm{SE}=1.26$ ). Hence, people responded faster in the blocks following the pretest block, although the absolute difference with the pretest was minimal for the incorrect responses. We observed the same trend for correct responses in the feedback condition (pretest: $\mathrm{M}=11.45 \mathrm{~s}, \mathrm{SE}=2.67$; intermediate: $\mathrm{M}$ $=3.67 \mathrm{~s}, \mathrm{SE}=0.33$; posttest: $\mathrm{M}=3.34 \mathrm{~s}, \mathrm{SE}=0.35$ ). However, the incorrect responses in the feedback condition showed a divergent pattern. Here, the average response time increased during the intermediate block $(\mathrm{M}=8.37 \mathrm{~s}, \mathrm{SE}=1.96)$, as compared to the pretest $(\mathrm{M}=6.92 \mathrm{~s}$, $\mathrm{SE}=1.34)$ or posttest $(\mathrm{M}=3.29 \mathrm{~s}, \mathrm{SE}=0.54)$. This indicates that the (negative) feedback was processed and made people take more time to respond. However, this additional deliberation time did not help (most participants) to arrive at the correct response (see previous section on response accuracy).

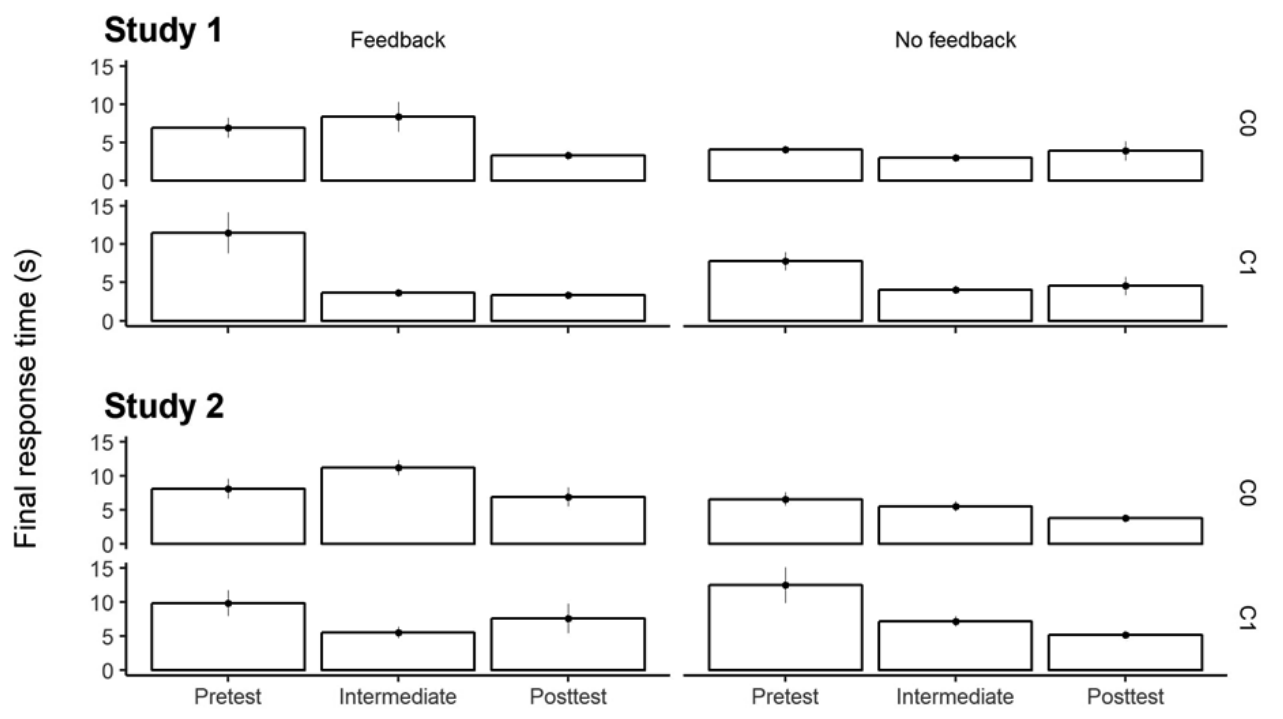

Block

Figure 6.3. Average final response times on conflict problems. $\mathrm{C} 0=$ incorrect performance, $\mathrm{C} 1=$ correct performance. Error bars are standard errors.

To examine more closely whether the increased response times for incorrect responses under feedback were related to accuracy, we simply contrasted the incorrect response latencies for reasoners who improved (i.e., posttest accuracy $>$ pretest accuracy, $n=8$ ) following 
feedback with those who did not improve $(n=26)^{7}$. Results indicated that improved reasoners had a longer deliberation time after receiving negative feedback $(\mathrm{M}=16.48 \mathrm{~s}, \mathrm{SE}=7.64)$ than the unimproved reasoners $(\mathrm{M}=5.87 \mathrm{~s}, \mathrm{SE}=0.74)$. The increase in comparison with the pretest was observed in both groups (improved reasoners: $\mathrm{M}=7.45 \mathrm{~s}, \mathrm{SE}=7.73$; unimproved reasoners: $\mathrm{M}=0.37 \mathrm{~s}, \mathrm{SE}=1.33$ ), although it was very small for unimproved reasoners. The interested reader can find an overview of the performance patterns of the improved reasoners in Figure S6.3 (Supplementary Material).

Conflict detection. In further exploratory analyses we used participants' final response latencies as proxy of conflict detection ${ }^{8}$. As noted in the introduction, previous studies have suggested that biased reasoners often show some minimal error or conflict sensitivity (Bago \& De Neys, 2017, 2019; De Neys, 2012; Frey et al., 2018; Johnson et al., 2016; Pennycook et al., 2015a). These studies contrast people's processing of conflict and no-conflict problems. On the no-conflict problems, the intuitively cued heuristic is also correct. On the conflict problems, the intuitively cued heuristic response conflicts with the correct response. If people are sensitive to this conflict, this should affect their processing (e.g., response time). Results indeed show that response times on incorrectly solved conflict problems are typically longer than the response times for correctly solved no-conflict problems (Bago \& De Neys, 2017, 2019; De Neys, 2012; Frey et al., 2018; Johnson et al., 2016; Pennycook et al., 2015a).

We first checked whether we replicated the results from previous studies. Overall, across both our conditions we found that during the pretest incorrect conflict response $(\mathrm{M}=5.32 \mathrm{~s}$ $\mathrm{SE}=0.66)$ indeed took longer than the correct no-conflict response $(\mathrm{M}=3.85, \mathrm{SE}=0.20$; i.e., on average a $1.47 \mathrm{~s}$ increase). The majority of biased reasoners showed this effect $(n=$ 60 out of $89,67.4 \%$ ). Next, analogous to the analysis of overall response times, we contrasted the conflict detection effects for each individual (i.e., mean response time incorrect conflict mean response time correct no-conflict) in the three consecutive blocks, per condition. Figure 6.4 (top panel) gives a complete overview. As with the overall latency effect, feedback clearly boosted the conflict detection effect during the intermediate block. This suggests that the increased deliberation time under feedback was specifically tied to the presence of conflict.

Finally, we also wanted to explore whether one's conflict detection was predictive of the intervention effect. That is, we examined whether reasoners whose accuracy improved from pretest to posttest already showed a stronger conflict detection effect in the first pretest block than those who had not improved (see Table 6.1, top panel). This indeed seemed to be case, in both the feedback and no-feedback conditions, the improved reasoning group had a relatively larger conflict detection effect size average at the pretest when compared to the unimproved reasoning group.

\footnotetext{
${ }^{7} \mathrm{~A}$ total of 5 improved participants and 11 unimproved participants in the feedback condition did not enter any incorrect responses in the intermediate (feedback) block and were thus not included in this analysis.

${ }^{8}$ Two-response studies have suggested that initial response latencies might not reliably track conflict detection effects at the initial response stage (Bago \& De Neys, 2017; Thompson \& Johnson, 2014).
} 


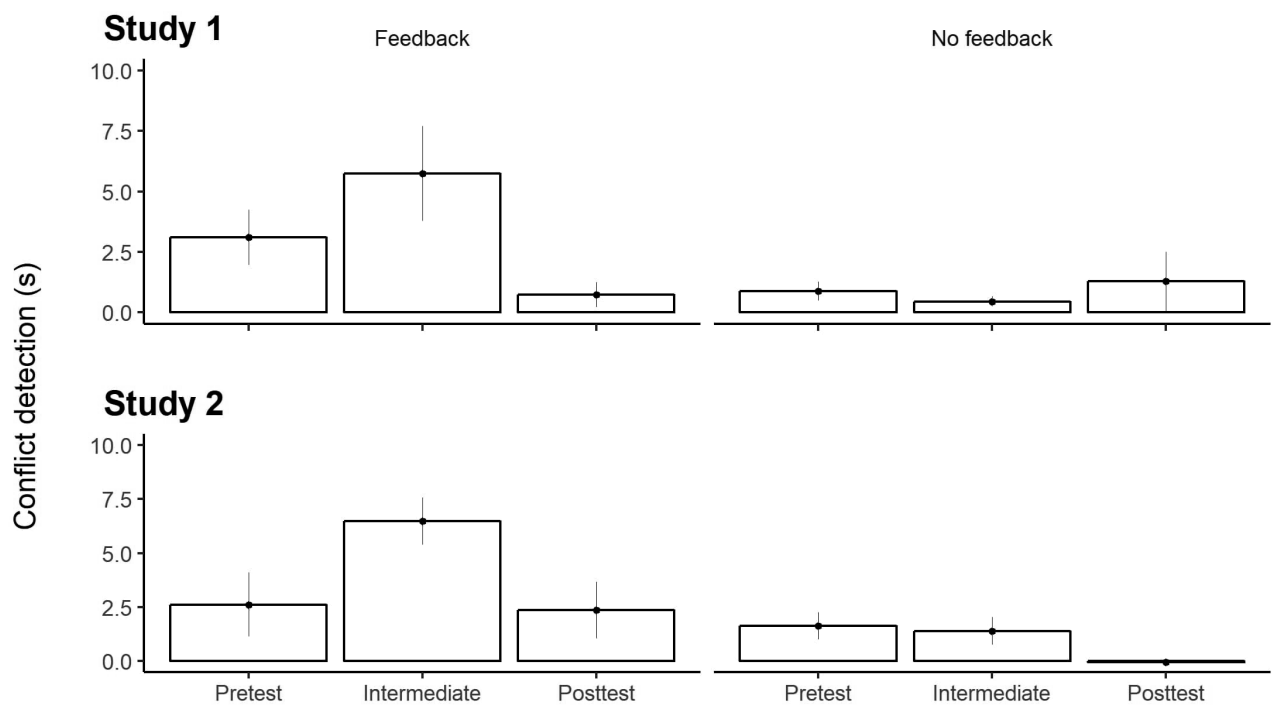

Block

Figure 6.4. Average conflict detection effect size as indexed by response time. Error bars are standard errors. Note. Due to a technical failure, final response time of one no-conflict trial in the intermediate block and one no-conflict trial in posttest block is missing for all participants in Study 2.

Table 6.1. Overview of average pretest conflict detection effect size as indexed by response time for improved versus unimproved biased reasoners

\begin{tabular}{lccc}
\hline & $n$ & $\mathrm{M}$ & $\mathrm{SE}$ \\
\hline Study 1 & & & \\
Feedback condition & & & \\
$\quad$ Improved reasoners & 13 & $5.55 \mathrm{~s}$ & 2.83 \\
$\quad$ Unimproved reasoners & 26 & $1.88 \mathrm{~s}$ & 0.91 \\
No-feedback condition & & & \\
$\quad 12$ & $2.54 \mathrm{~s}$ & 1.42 \\
$\quad$ Improved reasoners & 38 & $0.34 \mathrm{~s}$ & 0.20 \\
$\quad$ Unimproved reasoners & & & \\
Study 2 & & & 5.73 \\
Feedback condition & 9 & $9.44 \mathrm{~s}$ & 0.35 \\
$\quad$ Improved reasoners & 27 & $0.74 \mathrm{~s}$ & \\
$\quad$ Unimproved reasoners & & & 2.65 \\
No-feedback condition & 7 & $3.15 \mathrm{~s}$ & 0.36 \\
$\quad$ Improved reasoners & 25 & $1.21 \mathrm{~s}$ & \\
$\quad$ Unimproved reasoners & & & \\
\hline
\end{tabular}

Note. The $n$ s do not add up to the total sample sizes because 23 unimproved participants in Study 1 and 12 unimproved participants in Study 2 had a ceiled pretest performance (i.e., conflict detection effect size can only be calculated for participants who gave at least one biased response). 


\subsection{STUDY 2}

With Study 2, we aimed to test the robustness of the Study 1 findings while applying a number of methodological optimizations. First, we adopted a full experimental design instead of a quasi-experimental design and randomly allocated participants to a feedback and no-feedback condition. Second, we adopted a free-response format instead of multiple-choice answering options, to eliminate the possibility that a potential effect of feedback was driven by reasoning backwards from the presented answering options. Third, after the last problem in the posttest, participants were asked to justify their answer, to see whether they were able to explain the reasoning behind their answer. Fourth, participants had to solve two transfer problems after completing the bat-and-ball problems, to see whether a potential feedback effect also led to transfer.

\subsubsection{METHOD}

Given the similarity of both study designs and to avoid repetition, we only describe the aspects of the method that deviated from Study 1.

Pre-registration. The study design, sample size, and hypotheses were preregistered on the Open Science Framework (https://osf.io/b9u8r/). No specific analyses were preregistered.

Participants. For Study 2, 80 participants (all gave informed consent) were recruited on Prolific Academic (www.prolific.ac), were paid at a rate of $£ 5 / \mathrm{h}$, and were randomly assigned to either the feedback condition $(n=40)$ or the no-feedback condition $(n=40)$. All participants were native English speakers (49 females; age: $\mathrm{M}=34.3$ years, $\mathrm{SD}=12.6$ ). For most participants the highest completed level of education was high school $(45.0 \%)$ or a Bachelor degree (35.0\%), followed by a Master's degree $(8.8 \%)$, less than high school $(7.5 \%)$, and a Doctoral degree $(3.8 \%)$, respectively.

Materials. In addition to the 33 problems in Study 1, we administered one justification question and two transfer problems.

Justification. After completing their final bat-and-ball problem, participants received the following message (see Bago \& De Neys, 2019):

You have almost completed Block 3. We are interested in the reasoning behind your response to the final question:

In a school there are 130 boys and girls in total.

There are 100 more boys than girls. How many girls are there?

Could you please justify, why do you think that your previously entered response is the correct response to the question? Please choose from the presented options below:

o I did the math. Please specify how:

o I guessed

o I decided based on intuition/gut feeling

o Other, please specify: 
Two independent raters (first and second author) judged whether the specified justifications indicated a correct, incorrect, or unspecified justification. They were in agreement in $100 \%$ of the cases. A justification was coded as correct when the correct calculation was provided (e.g., " $130-100=30 / 30 / 2=15 ")$. A justification was coded as incorrect when it referred to the incorrect/heuristic math (e.g., "The total number is 130 and the boys are 100 more so I subtracted 100 from the total to give me the answer"). Justifications that did not explain the math procedure were coded as "not specified" (e.g., "I DID THE MATH").

Transfer problems. The transfer problems were two CRT-like items. The first one was an adapted version of the "widget" problem from the original CRT (Frederick, 2005):

If it takes 10 minutes for ten cooks to prepare 10 hamburgers,

how long would it take for 200 cooks to prepare 200 hamburgers?

The heuristic response here is 200 minutes and the correct response 10 minutes. The second transfer problem was an item derived from Thomson and Oppenheimer (2016):

If you're running a race and you pass the person in second place, what place are you in?

Here, the heuristic response is "first place" and the correct response "second place".

Procedure. Study 2 used a free response format, following the same procedure as Bago and De Neys (2019). Both in the initial and final response stage, participants needed to click on a blank field where they had to enter their response, type their answer, and click on a button labelled "Next" to advance. Because typing an answer requires more time than selecting a multiple-choice answer, the response deadline was $8000 \mathrm{~ms}$ instead of $5000 \mathrm{~ms}$ (see Bago \& De Neys, 2019). After $6000 \mathrm{~ms}$ the background of the screen turned yellow to warn participants about the upcoming deadline. Participants were instructed to only type numbers (no letters). Each time after a participant had entered an invalid character during the intuitive response stage, (s)he received a reminder message to only enter numbers. All invalid responses were excluded. In the final response stage it was not possible to enter other characters than numbers. To familiarize participants with the two-response procedure, they first solved two unrelated problems with the initial response deadline but yet without the load task. Next they practiced one load task and, thereafter, they solved two problems following the complete two-response procedure.

The order of all 33 problems was randomized in the same way as in Study 1 except for the fact that all participants completed the same final problem, a conflict problem, so that we could ask for their justification.

After the justification question, participants were instructed that they had completed block 3 out of 4 and that the final block consisted of only two problems that were somewhat different from the previous ones and, additionally, that they could think as long as they wanted to solve these. In contrast to Study 1, we did not check whether participants were familiar with the classic bat-and-ball problem (as we did not use this as an exclusion criterion, see Footnote 4). 


\section{FEEDBACK ON THE BAT-AND-BALL PROBLEM}

Feedback manipulation. We made the accuracy feedback during the intermediate block somewhat more salient than in Study 1. That is, for correct answers the feedback said "CORRECT answer!" in green, bold, capitalized letters and for incorrect answers "INCORRECT answer!" in red, bold, capitalized letters.

Exclusion criteria. We analyzed all conflict and no-conflict trials, which were 30 trials $\times$ 80 participants $=2400$ trials in total. Participants failed to provide their first answer before the deadline on 37 trials (1.4\% of all trials) and further failed to pick the correct matrix for the load task on 269 trials (11.4\% of remaining trials). Since we could not guarantee that the initial response for these trials did not involve any deliberation, we discarded them and analyzed the 2094 remaining trials $(87.3 \%$ out of 2400 trials). On average each participant contributed 13.1 $(\mathrm{SD}=1.6)$ conflict trials and 13.1 $(\mathrm{SD}=1.5)$ no-conflict trials.

\subsubsection{RESULTS AND DISCUSSION}

In general, the results of Study 2 were highly similar to the results of Study 1. For completeness, we discuss all results but try to be concise where possible.

Response accuracy. Figure 6.1 (bottom panel) provides an overview of the average performance of the feedback and no-feedback condition on the conflict problems. For the final accuracies, the average performance at the pretest was $15.1 \%(\mathrm{SE}=5.4)$ for the feedback condition and $28.8 \%(\mathrm{SE}=6.7)$ for the no-feedback condition. Both conditions improved in average performance from pretest to posttest, but the feedback condition improved, with an average increase of $14.9 \%(\mathrm{SE}=4.9)$, more than the no-feedback condition, which had an average increase of $7.7 \%(\mathrm{SE}=3.8)$. The ANOVA indicated that reasoners in both conditions improved their accuracy from pretest to posttest but were not affected by the feedback manipulation, block: $F(1,78)=13.25, p<.001, \eta_{\mathrm{p}}^{2}=.211$; condition: $F(1,78)=1.26, p=$ $.265, \eta_{\mathrm{p}}^{2}=.048$; block $\times$ condition: $F(1,78)=1.35, p=.248, \eta_{\mathrm{p}}^{2}=.01$.

As in Study 1, the initial accuracies showed the same pattern as the final accuracies but with slightly lower performance averages (see also Figure 6.1). The average initial accuracy at the pretest was $10.0 \%(\mathrm{SE}=4.1)$ for the feedback condition and $18.3 \%(\mathrm{SE}=5.2)$ for the no-feedback condition. Both conditions improved in average performance from pretest to posttest, but the feedback condition improved, with an average increase of $17.5 \%$ ( $\mathrm{SE}=$ 5.3 ), more than the no-feedback condition, which had an average increase of $15.3 \%$ ( $\mathrm{SE}=$ 4.9). Again, the ANOVA indicated that reasoners in both conditions improved their accuracy from pretest to posttest but were not affected by the feedback manipulation, block: $F(1,78)=$ $20.87, p<.001, \eta_{\mathrm{p}}^{2}=.243$; condition: $F(1,78)=0.83, p=.364, \eta_{\mathrm{p}}^{2}=.048$; block $\times$ condition: $F(1,78)=0.10, p=.754, \eta_{\mathrm{p}}^{2}=.007$.

The no-conflict control problems also showed the expected pattern. That is, we observed a performance at ceiling for both conditions in all blocks with grand means of $98.6 \%$ ( $\mathrm{SE}=$ $0.4)$ and $99.7 \%(\mathrm{SE}=0.2)$ for initial and final accuracy, respectively (see also Figure S6.1 in the Supplementary Material). An ANOVA on initial accuracy showed that there was a small main effect of block, $F(1,78)=7.03, p=.010, \eta_{\mathrm{p}}^{2}=.083$, no effect of condition, condition: $F(1,78)=0.37, p=.548, \eta_{\mathrm{p}}^{2}=.004$, or an interaction, $F(1,78)=0.01, p=.933, \eta_{\mathrm{p}}^{2}<.001$. For final accuracies none of the factors reached significance, block: $F(1,78)<0.001, p>.999$, 
$\eta_{\mathrm{p}}^{2}<.001$; condition: $F(1,78)<0.001, p \geq .999, \eta_{\mathrm{p}}^{2}<.001$; block $\times$ condition: $\mathrm{F}(1,78)=2.00$, $p=.161, \eta_{\mathrm{p}}^{2}=.025$.

Thus, also when using a free-response format (Study 2) instead of multiple-choice options (Study 1), reasoners improved their accuracy on the conflict bat-and-ball problems from pretest to posttest but were not affected by the feedback manipulation per se (despite a small observed trend towards a better improvement for the feedback condition $)^{9}$.

Individual level directions of change. Figure 6.2 (bottom panel) plots the direction of change classification on each of the consecutive conflict problems for each individual participant. Just as in Study 1, the figure shows a very similar pattern in the feedback and no-feedback conditions. First, most participants (53 out of 80 participants or $65.0 \%$ ) were in the "biased" group. That is, they predominantly gave incorrect intuitive and deliberate (00-) responses and remained biased throughout the study. Second, $17.5 \%$ of the participants (14 out of 80 ) was in the "correct" group, meaning that they already gave a correct (final) response at their very first trial and predominantly remained responding correctly throughout the study. Third, $17.5 \%$ (14 out of 80 ) was in the "insight" group. These participants started with an incorrect response and found the correct answer somewhere along the way, and remained correct from then on. Again, for both the "correct" and "insight" group, we observed that most participants who found the correct answer automatized the reasoning very quickly (i.e., only on or two 01-responses preceded the consistent row of 11-responses).

With regard to the comparison between conditions, 5 participants in the feedback condition (12.5\% of total $n=40)$ showed the insight pattern after the pretest block (i.e., after onset of feedback), compared to 1 participant $(2.5 \%$ of total $n=40)$ in the no-feedback condition. Hence, in line with Study 1, we observed evidence for a small intervention trend but this trend is driven by only a handful of participants. The vast majority of biased reasoners was not affected by feedback (or mere repeated presentation in the no-feedback condition).

Response latencies. Figure 6.3 (bottom panel) provides an overview of the average final response times of the feedback and no-feedback condition on the conflict problems (see Figure S6.2 in the Supplementary Material for an overview of response time on the no-conflict problems). For consistency with Study 1, we again excluded all trials with final response times $>120 \mathrm{~s}$. This concerned six conflict trials and two no-conflict trials. We found a similar pattern as in Study 1. In the no-feedback condition we observed a general speeding-up after the pretest block, both for correct responses (pretest: $\mathrm{M}=12.45 \mathrm{~s}, \mathrm{SE}=2.60$; intermediate: $\mathrm{M}=7.14 \mathrm{~s}, \mathrm{SE}=0.78$; posttest: $\mathrm{M}=5.20 \mathrm{~s}, \mathrm{SE}=0.34$ ) and incorrect responses (pretest: $\mathrm{M}$ $=6.58 \mathrm{~s}, \mathrm{SE}=0.98$; intermediate: $\mathrm{M}=5.51 \mathrm{~s}, \mathrm{SE}=0.77$; posttest: $\mathrm{M}=3.79 \mathrm{~s}, \mathrm{SE}=0.28$ ). We observed the same trend for correct responses in the feedback condition (pretest: $\mathrm{M}=$ $9.80 \mathrm{~s}, \mathrm{SE}=1.91$; intermediate: $\mathrm{M}=5.50 \mathrm{~s}, \mathrm{SE}=0.83$; posttest: $\mathrm{M}=7.58 \mathrm{~s}, \mathrm{SE}=2.11$ ) but not for the incorrect responses. The incorrect responses in the intermediate feedback block again showed increased response time (intermediate: $\mathrm{M}=11.18 \mathrm{~s}, \mathrm{SE}=1.12$ ), as compared to the other two blocks (pretest: $\mathrm{M}=8.12 \mathrm{~s}, \mathrm{SE}=1.48$; posttest: $\mathrm{M}=6.93 \mathrm{~s}, \mathrm{SE}=1.36$ ).

\footnotetext{
${ }^{9}$ As in Study 1, we conducted some additional (explorative analyses) to be absolutely sure that the feedback had no effect (see Footnote 5). These analyses again yielded no effect of feedback.
} 
This indicates that the (negative) feedback was processed and made people take more time to respond. However, this additional deliberation time did not help (most) participants to arrive at the correct response (see previous section on response accuracy). Again, we found that the improved reasoners had longer response times $(n=6, \mathrm{M}=17.10, \mathrm{SE}=3.40)$ after receiving negative feedback than those who had not improved $(n=27, \mathrm{M}=9.86, \mathrm{SE}=1.01)^{10}$. The increase in comparison with the pretest, however, was observed in both groups (improved reasoners: $\mathrm{M}=10.75 \mathrm{~s}, \mathrm{SE}=3.21$; unimproved reasoners: $\mathrm{M}=3.78 \mathrm{~s}, \mathrm{SE}=0.85$ ) although it was again smaller for the unimproved reasoners (an overview of the improved reasoners' performance pattern is shown in Figure S6.3 in the Supplementary Material).

Conflict detection. Consistent with Study 1, we replicated the conflict detection effect as found in previous studies (e.g., Bago \& De Neys, 2019; Frey et al., 2017). Across both our conditions we found that during the pretest, incorrect conflict responses $(\mathrm{M}=7.39 \mathrm{~s} \mathrm{SE}$ $=0.91)$ indeed took longer than the correct no-conflict responses $(\mathrm{M}=5.51, \mathrm{SE}=0.29$; i.e., on average a $1.88 \mathrm{~s}$ increase). The majority of biased reasoners showed this detection effect ( $n=48$ out of $68,70.6 \%$ ). Figure 6.4 (bottom panel) gives a complete overview of the conflict detection effects in the three consecutive blocks per condition. As with the overall latency effect and consistent with what we observed in Study 1, feedback clearly boosted the conflict detection effect during the intermediate block. Finally, we again also found that one's conflict detection was predictive of the intervention effect (see Table 6.1, bottom panel). In both the feedback and no-feedback condition, the improved reasoning group had a relatively larger conflict detection effect size average at the pretest when compared to the unimproved reasoning group.

Justification. The interested reader can find an overview of response justifications analysis in the Supplementary Material Table S6.5. Results showed that almost all participants that had solved the last conflict item correctly also gave the correct math justification (feedback condition: 11 out of 13; no-feedback condition: 14 out of 14). The majority of the participants that solved the item incorrectly, gave an incorrect math justification (feedback condition: 16 out of 24; no-feedback condition: 18 out of 25 ). Hence, irrespective of feedback, people who responded correctly typically also managed to explicate the correct solution strategy.

Transfer problems. For each participant, we calculated the average proportion of correct responses on the two transfer problems. Overall, average performance on the transfer problems was more or less similar in the feedback $(\mathrm{M}=46.3 \%, \mathrm{SE}=6.6 \%)$ and no-feedback condition $(\mathrm{M}=52.5 \%, \mathrm{SE}=6.7 \%)$. We also explored whether the transfer problem performance differed for improved versus unimproved reasoners. As Table S6.6 in Supplementary Materials shows, this was indeed the case. Both in the feedback and no-feedback condition, an improved reasoner $(n=16, \mathrm{M}=59.4 \%, \mathrm{SE}=9.4 \%)$ was more likely to solve the transfer problems correctly than an unimproved reasoner $(n=64, \mathrm{M}=46.88 \%, \mathrm{SE}=5.3 \%)^{11}$.

\footnotetext{
${ }^{10} \mathrm{~A}$ total of 3 improved participants and 4 unimproved participants in the feedback condition did not enter any incorrect responses in the intermediate (feedback) block and were thus not included in this analysis.

${ }^{11}$ We also ran a control analysis in which reasoners who were at ceiling in the pretest $(n=12)$ were excluded from the unimproved group. Results led to the same conclusion (see Table S6.6).
} 


\subsection{GENERAL DISCUSSION}

In the present two studies we tested the impact of a minimal intervention - response accuracy feedback - on people's bat-and-ball performance. We presented participants 15 standard (conflict) and 15 control (no-conflict) versions of the bat-and-ball problem, in three consecutive blocks (pretest, intermediate, and posttest). Half of the participants received accuracy feedback during the intermediate block, whereas the other half did not. Overall, the results of both studies were very consistent and clearly indicated that feedback had, on average, no significant effect on participants' bat-and-ball accuracy. We only observed a small trend in a handful of participants. Our explorative analyses did reveal a trend towards a feedback effect on response latencies (i.e., longer response times after receiving negative feedback) and conflict detection (i.e., larger conflict detection effect after receiving negative feedback). Hence, it seemed that feedback evoked extra deliberation but did not help most participants to arrive at the correct response. Interestingly, the small group of reasoners who did learn to correct their errors after receiving feedback, showed a stronger conflict detection effect in the pretest block, took more deliberation time after receiving negative feedback, and performed better on the two transfer problems (in Study 2), compared to the group of reasoners that remained biased on all problems.

Why was the feedback not more effective for improving bat-and-ball accuracy? One suggestion is related to the nature of error on this task. Previous studies (and the current study) revealed that the majority of the biased bat-and-ball reasoners already show some minimal error or bias detection from the onset (Bago \& De Neys, 2019; Frey et al., 2018; Gangemi et al., 2015; Hoover \& Healy, 2019; Mata, 2019; Mata et al., 2014, 2017; Travers et al., 2016). Hence, even without feedback people at least seem to implicitly detect that their answer is not fully warranted. In this light, it is perhaps not surprising that telling them this explicitly has little effect. In other words, people are not biased because they do not realize that 10 cents is incorrect but rather because they do not know how to arrive at the correct solution strategy. Given that most people are capable of solving the algebra behind the bat-and-ball problem (Hoover \& Healy, 2017), it remains an open question why they do not arrive at the correct solution strategy themselves. One possible explanation is that the required algebraic solution strategy concerns a reasoning strategy that is not frequently used in the daily-life reasoning of most people and is thus less easily available in long-term memory. Hence, a much stronger or more informative retrieval cue would be needed to arrive at the algebraic strategy. In this sense, giving people more detailed feedback or tutoring about the correct solution strategy might prove more effective.

Nevertheless, in addition to the large majority that remained biased, our results (and Raoelison \& De Neys, 2019) also show that some individual participants do manage to arrive at the correct solution strategy themselves. Here, we can distinguish between participants that were right from the start (the "correct" group) and participants that managed to correct themselves after responding biased at first (the "insight" group). For the "insight" group, we believe that our results on the predictive conflict detection effects are especially interesting. Previous studies have suggested there are individual differences in the extent of the conflict 
signal (Bago et al., 2019; Bago \& De Neys, 2017, 2019; Frey et al., 2018; Pennycook et al., 2015a). The current results indicated that those reasoners who improved from pretest to posttest (through the feedback or through mere repeated exposure), already showed a more pronounced conflict detection effect (i.e., higher latency increase) before the intervention. Hence, for those with the strongest signal, a minimal intervention "nudge" did suffice to get them to start reasoning correctly. In other words, the people who strongly feel that the 10 cents is incorrect are more likely to arrive at the solution strategy themselves after only a small push to think a little further. Following our previous "availability" suggestion, this group might show stronger conflict detection at the start and could correct themselves because the proper algebraic solution strategy was more easy available in long-term memory than for those who remained biased (e.g., because they have better numeracy skills, greater cognitive ability, or use the skill more often in daily life). This may also explain why they performed better on the transfer tasks than the unimproved "biased" group.

Finally, the "correct" group obviously did not need any intervention to arrive at the correct answer, and was therefore not affected by the feedback manipulation or the repeated problem presentation. We suggest that the solution strategy was most easily available for this correct group, which is in line with previous research on the CRT, showing that those with better numeracy skills, greater cognitive capacity, or work in a domain that requires numeracy skills are more likely to perform correctly (Campitelli \& Gerrans, 2014; Janssen et al., 2019; Toplak et al., 2011, 2014).

Obviously, the limited average impact of our feedback intervention does not entail that it is impossible to tutor people on the bat-and-ball problem. Our goal in the present study was to focus on a simple intervention that has proven to be effective in other fields. As we alluded to above, one could try to boost efficacity by switching to a more extensive tutoring or training in which participants are informed about how to arrive at the correct solution strategy. For example, Hoover and Healey (2017) showed that properly instructing people about the underlying mathematical equation can help to boost performance in the bat-and-ball problem. In this light, it might be interesting to explore the impact of reasoning feedback that may guide participants' reasoning towards the required calculation (e.g., explaining and highlighting the role of the "more than" phrase, adding the correct equation, etc.). Furthermore, although the simple response feedback was not an effective de-biasing strategy for the bat-and-ball problem, it would be interesting to explore its impact on other problems, for example on the classic base-rate problem (De Neys et al., 2011). In this problem the correct solution strategy is based on a very elementary statistical rule which is easy available in long-term memory of most people. If our suggestion concerning the ease of availability of a solution strategy is true, then accuracy feedback could have a stronger de-biasing impact here.

The current research had several limitations. First, the observed trends in both studies suggest that there might actually be a small effect of feedback that we were unable to detect with current sample size (which only allowed for picking up on medium effects). Second, accuracy feedback was given while participants were still burdened with the load task, which might have had an effect on their attention to the feedback. Hence, even though the response latencies suggested that participants processed the negative feedback, it could be that the 


\section{CHAPTER 6}

feedback would have been more effective when presented after the load task. Third, the overall obtained accuracies were rather low in comparison with other studies (Brañas-Garza et al., 2019), which might indicate that the current participants were not motivated enough to improve their reasoning. In this light, (monetary) incentives would perhaps have made the feedback more effective, although a recent meta-analysis indicated that monetary incentives have no overall effect on bat-and-ball performance (Brañas-Garza et al., 2019).

In closing, although the overall observed impact of feedback on people's accuracy was very limited, we believe that the present paper does indicate that a feedback manipulation has potential as a methodological tool and warrants to be further explored in the reasoning and decision making field. 
FEEDBACK ON THE BAT-AND-BALL PROBLEM 


\section{Psychometric properties of the Actively}

\section{Open-Minded Thinking scale}

An adapted version of this chapter was published as:

Janssen, E. M., Verkoeijen, P. P. J. L., Heijltjes, A. E. G., Mainhard, T., Van Peppen, L. M., \& Van Gog, T. (2020). Psychometric properties of the Actively Open-Minded Thinking scale. Thinking Skills and Creativity, 36, 100659. doi:10.1016/j.tsc,2020.100659

\section{Acknowledgement of author contributions:}

$\mathrm{EJ}, \mathrm{PV}$, and $\mathrm{AH}$ designed the study, AH recruited participants and collected the data, EJ analyzed the data, TM checked the data package, EJ drafted the manuscript, all authors contributed to critical revision of the manuscript, TM, PV, AH, and TvG supervised the study. Additional note: EJ would like to thank Renske Kuipers for her assistente with data analysis. 


\section{ABSTRACT}

The Actively Open-minded Thinking scale (AOT; Stanovich \& West, 2007) is a questionnaire that is used to measure the disposition towards rational thinking as a single psychological trait. Yet, despite its frequent use, also in abbreviated form, it is still unclear whether sumscores of the AOT can actually be used to order individuals on their disposition towards actively open-minded thinking and whether the questionnaire can be validly shortened. The present study aimed to obtain a valid and shorter AOT. We conducted Mokken scale analyses on the (Dutch) AOT using two samples of higher education students $(N=930 ; N=509)$. Our analyses showed that none of the 41 items could discriminate sufficiently between respondents with varying latent trait levels. Furthermore, no item-set of the AOT could be obtained to validly order individuals on the assumed latent trait, which is a crucial assumption when using it in research. Consequently, it is questionable whether scores on the AOT provide insights into the concept it aims to measure.

Keywords. actively open-minded thinking; thinking dispositions; rational thinking; reasoning and decision-making; psychometrics; assessment; mokken scale analysis. 


\subsection{INTRODUCTION}

After Baron (1991) introduced the concept of actively open-minded thinking, the Actively Open-minded Thinking scale (AOT) developed by Stanovich and West $(1997,2007)$ has been widely used to measure people's disposition towards rational thinking (see Table 7.1). The AOT has been shown to predict performance on, for example, critical thinking tests and is an important measure in reasoning and decision-making research (see e.g., Heijltjes et al., 2014; Toplak et al., 2011; West et al., 2008). The most widely used version of the AOT (Stanovich \& West, 2007) consists of 41 statements in the form of Likert type items. Because this version takes substantial time to administer, it would be practical to obtain a valid shorter version (cf. the abbreviated versions to assess Need for Cognition, another widely used disposition scale; Cacioppo et al., 1984; Chiesi et al., 2018). In the literature, many different versions of the AOT have been applied, with item selections ranging from as few as 7 items to 47 items (see also Table 7.1).

Thus far, based on considerably high Cronbach's alpha's, most versions of the AOT including the original version, have been considered to be reliable. Despite the high Cronbach alpha's and the associations of the AOT scores with variables like critical thinking test performance, it is as yet unclear to what degree the items together reflect the single psychological trait of actively open-minded thinking. In other words, it is unclear what the internal validity of the AOT is and because of this, it is hard to substantively interpret correlations of the AOT with other variables. Thus, to further advance and strengthen research in the domain of reasoning and decision-making, it is important to investigate whether and to what degree the items included in the AOT measure the psychological trait actively open-minded thinking.

The little research that has been conducted on the internal validity of the AOT has used Factor Analysis (FA; Stanovich \& West, 1997; Svedholm-Häkkinen \& Lindeman, 2018). The results of these FAs, however, shed doubt on whether the AOT sumscores measure a single psychological trait and, thus, on whether AOT sumscores can be interpreted meaningfully; a finding we will elaborate on shortly. Furthermore, an assumption of FA is that the item scores are continuous and although this is a common assumption in social sciences research, psychometricians argue that Likert scale data are not continuous and should be treated as categorical data (Flora et al., 2012; Jamieson, 2004; Liddell \& Kruschke, 2018). An alternative test theory approach that is suitable for categorical data is Mokken scale analysis (Mokken, 1971; Sijtsma \& Molenaar, 2002). Further benefits of this analysis are that it does not require multivariate normality or linear correlation between items, which are two assumptions of FA that are easily violated when working with Likert scale data (Flora et al., 2012; Jamieson, 2004; Liddell \& Kruschke, 2018). Therefore, the present study applied Mokken scale analyses on (Dutch) AOT data (translated version used in Heijltjes et al., 2014), to see whether we could obtain a shorter and valid version that measures a single psychological trait. In addition, for comparability with prior work by Stanovich and West (2007) and by Svedholm-Häkkinen and Lindeman (2018) we also conducted two Confirmatory Factor Analyses (CFAs), applying their proposed models. 


\subsubsection{ACTIVELY OPEN-MINDED THINKING}

Baron (1991) introduced the concept of actively open-minded thinking as an ideal standard of good thinking. According to Baron "good thinking consists of (1) search that is thorough in proportion to the importance of the question, (2) confidence that is appropriate to the amount and quality of thinking done, and (3) fairness to other possibilities than the one we initially favor" (Baron, 2008, p. 200, italics added); open-minded refers to "the consideration of new possibilities, new goals, and evidence against possibilities that already seem strong" (Baron, 2008 , p. 200); and active refers to not waiting for these things to happen but seeking them out. In his work, Baron argued that our thinking often deviates from the ideal of actively open-minded thinking, which leads to biases in our reasoning and decision-making. Think for example of our tendency to ignore evidence that goes against the conclusion we favor (i.e., confirmation bias).

Actively open-minded thinking is viewed as a thinking disposition. Thinking dispositions (or cognitive styles) are viewed as relatively stable psychological mechanisms that tend to generate characteristic behavioral tendencies and tactics (Stanovich et al., 2016). Thinking dispositions reflect people's goal management, epistemic values, and epistemic self-regulation. In their book "The rationality quotient", Stanovich et al. (2016), claim that, next to intelligence, thinking dispositions underlie rationality. They argue that - independent of cognitive ability - those who habitually seek various points of view or think extensively about a problem tend to display more rational behavior than those without such thinking dispositions. Psychologists have studied many thinking dispositions in relation to rationality. For instance, an individual's tendency to engage in and enjoy thinking, measured with the Need for Cognition scale, (NFC; Cacioppo \& Petty, 1982; Cacioppo et al., 1984) has shown to be positively associated with rational thinking skills ${ }^{1}$ after controlling for variance due to cognitive ability (Toplak \& Stanovich, 2002; West et al., 2008). Other examples of thinking disposition questionnaires are the Rational-Experiential Inventory (Epstein et al., 1996) and the Consideration of Future Consequences scale (Strathman et al., 1994). The disposition to think actively open-minded, however, is theorized to be the most central to rational thinking (Baron, 2008; Stanovich et al., 2016).

\subsubsection{THE ACTIVELY OPEN-MINDED THINKING SCALE}

Baron (2008) mostly measured actively open-minded thinking qualitatively by assessing people's beliefs of good thinking, for example, through asking them to evaluate exemplars of the thinking of others. Inspired by Baron's work, Stanovich and West $(1997,2007)$ composed a questionnaire to measure actively open-minded thinking, the Actively Open-minded

\footnotetext{
${ }^{1}$ In these studies, rational thinking is operationalized in ability to avoid bias in reasoning and decision-making measured with performance on so-called heuristics-and-biases tasks (e.g., West et al., 2008). These tasks measure whether someone is prone to a specific bias during a specific type of reasoning.
} 
Thinking scale (AOT). The AOT consists of statements about thinking based on which participants rate their (dis)agreement on a Likert response format with six categories: 6 (agree strongly), 5 (agree moderately), 4 (agree slightly), 3 (disagree slightly), 2 (disagree moderately), 1 (disagree strongly). An example of such a statement is: "A person should always consider new possibilities."

For the first version in 1997, Stanovich and West (1997) composed 56 items distributed across eight subscales (Flexible Thinking, Openness Values, Dogmatism, Categorical Thinking, Openness-ideas, Absolutism, Superstitious Thinking, and Counterfactual Thinking). They found only three out of the eight subscales were to be reliable ${ }^{2}$. Moreover, a principal components analysis (PCA) revealed that the first six subscale sumscores formed one component explaining most of the variance (40.8\%). Consequently, they excluded the subscales Superstitious Thinking and Counterfactual Thinking and computed a single composite score using the remaining subscales (i.e., summing the scores on the Flexible Thinking, Openness-Ideas, and Openness-Values scales and subtracting the sum of the Absolutism, Dogmatism, and Categorical Thinking scales), hereby treating the AOT as a unidimensional trait without subfactors. This score intended to order respondents on a scale ranging from "openness to belief-change and cognitive flexibility" (high scores) to "cognitive rigidity and resistance to belief change" (low scores). In the 1997 study, the Spearman-Brown corrected split-half reliability of the scale was .90 and Cronbach's $\alpha$ of the scale as a whole was .88.

Ten years after the first introduction of the AOT (Stanovich \& West, 1997), Stanovich and West (2007) introduced a 41-item AOT, which from then became the most widely used version of the AOT. The scale consisted again of six subscales: four scales remained the same as in the first version (i.e., Flexible Thinking, Openness Values, Dogmatism, Categorical Thinking), but two scales (Openness-Ideas and Absolutism) were replaced with the subscales Belief Identification and Counterfactual thinking. A sumscore of the 41 items (after reverse scoring of 30 items) intended to order respondents on their disposition towards actively open-minded thinking. Again, the reliability for the total scale was good (split half reliability .75 and Cronbach's alpha .83). Because the subscale reliabilities were not reported, we assume that the new item selection was again intended to assess actively open-minded thinking as a unidimensional trait. However, an analysis to test this assumption was not reported.

\subsubsection{RESEARCH USING THE ACTIVELY OPEN-MINDED THINKING SCALE}

Since its introduction, numerous researchers have used the AOT. For example, multiple studies showed that the sumscores of this scale positively correlated with rational thinking (Sá et al., 1999, 2005; Sá \& Stanovich, 2001; Toplak et al., 2011; West et al., 2008; see also Heijltjes et al., 2014 who used a Dutch translation; Lean Keng \& AlQudah, 2017 who used an Arabic

\footnotetext{
${ }^{2}$ Split-half reliability and Cronbach's alpha were, respectively, .49 and .50 for Flexible thinking; .73 and .71 for Openness values; .54 and .60 for Dogmatism; not reported for Categorical thinking because the scale consisted of only 2 items; .73 and .77 for Openness-ideas; and .69 and .64 for Absolutism, not reported for Counterfactual thinking because the scale consisted of only 2 items; .73 and .73 for Superstitious thinking (see Stanovich \& West, 1997).
} 
translation; or Svedholm-Häkkinen \& Lindeman, 2018 who used a Finnish translation). A search in Web of Science (February 2019) indicated that the scientific papers introducing the first (Stanovich \& West, 1997) and second version of the AOT (Stanovich \& West, 2007) have been cited in 205 and 87 journal articles, respectively. We reviewed the 87 studies citing the 2007 version (currently the most widely used version) and found that 36 had adopted (a part of) the AOT as a measure (see Table 7.1). Researchers used it - often in combination with other disposition questionnaires - as a predictor of reasoning and decision-making (e.g., performance on rational, scientific, or analytic reasoning tasks or political choices), epistemic beliefs (e.g., evolutionary theory acceptance, belief in conspiracy theories, or religiosity), or behavior (e.g., being a gambler or showing adaptive teaching behavior). Studies varied widely, however, in the way the scale was administered. Some relatively small differences concerned the response formats (4-point to 7-point Likert scales) and whether the items were intermixed with other (disposition) questionnaires. A more important difference concerned the item selection. Within those 36 studies, 12 studies used a different item selection than the original 41-item AOT (see Table 7.1). In addition, in their book 'The Rationality Quotient' Stanovich et al. (2016) introduced a 30-item version and a 16-item short-form. Most studies did report a sufficient reliability for the total scale, but as listed in Table 7.1, none tested the factor structure. Hence, it is still an open question whether and to what degree the AOT from 2007 and the newer versions measure actively open-minded thinking as a unidimensional trait.

Recently, Svedholm-Häkkinen and Lindeman (2018) noted that it is not clear whether the AOT is unidimensional or multidimensional because the PCA on the first 47-item AOT (Stanovich \& West, 1997) was run on sumscores of the subscales rather than single items and because subsequent studies reported reliability measures for the scale as a whole only. Svedholm-Häkkinen and Lindeman (2018) aimed to develop a reliable, valid, shorter AOT and investigated whether the AOT was multidimensional or not. To this end, they conducted FAs in four separate samples $(\mathrm{N}=2735, \mathrm{~N}=458, \mathrm{~N}=102$, and $\mathrm{N}=50)$ who had completed a Finnish version of the 41-item AOT (Stanovich \& West, 2007). A 17-item version was sufficient to obtain a good reliability (Cronbach's alpha) for the total scale and to obtain correlations with variables assessing other thinking dispositions, social competence, and supernatural beliefs. However, their results also showed that the AOT was not unidimensional. They compared five different factor models and concluded that four intercorrelated subfactors (Dogmatism, Fact resistance, Liberalism, and Belief personification) described the data best. Neither a model with a higher-order factor (i.e., representing active open-mindedness) explaining the common variance in the four subscales, nor a single factor solution described the data adequately, which suggests that AOT sumscores cannot be used to validly order individuals on the assumed psychological trait of active open-mindedness. In addition, just as in the study by Stanovich and West (1997), the four subscales were only marginally or not reliable ${ }^{3}$. 


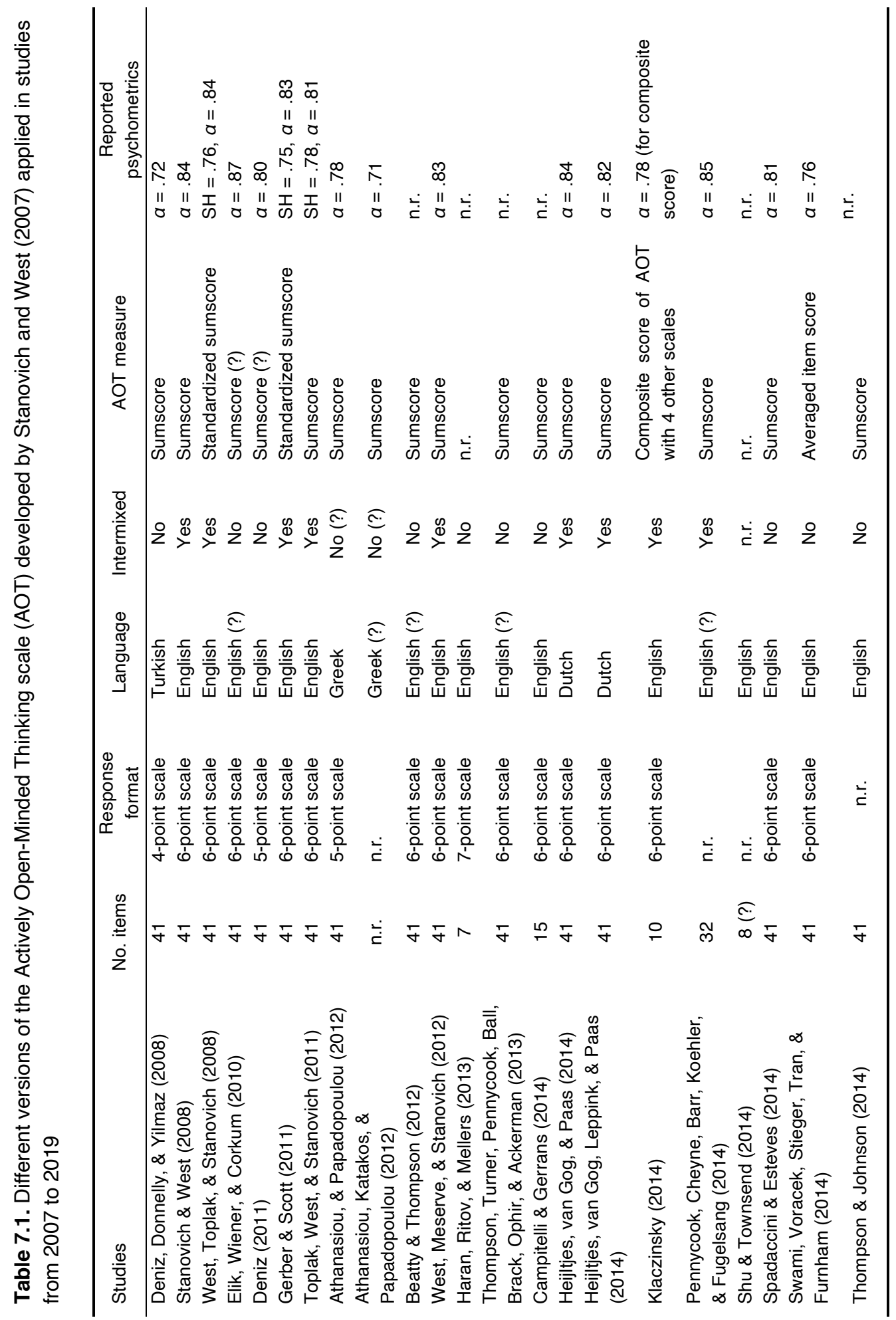




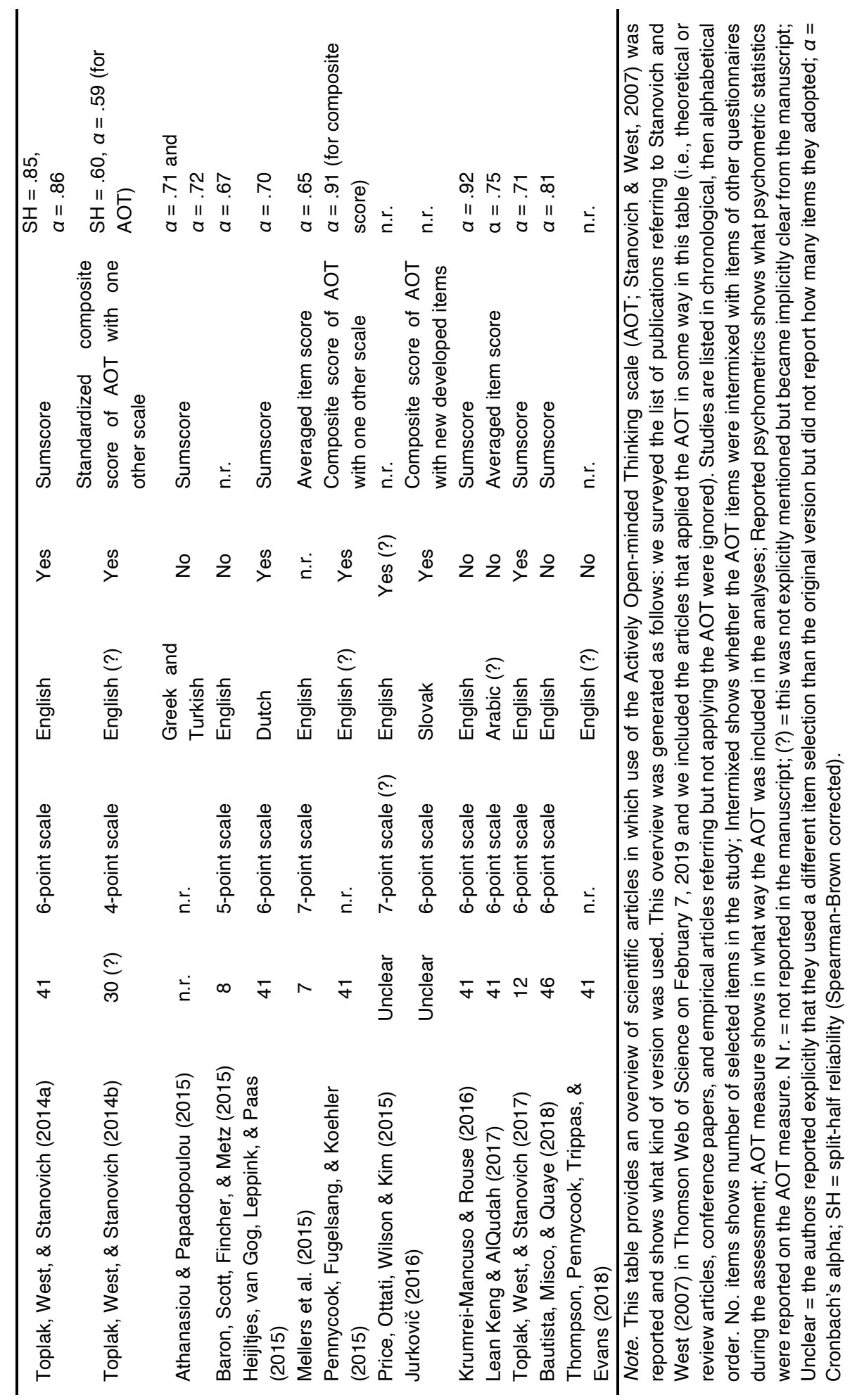




\subsubsection{THE PRESENT STUDY}

In sum, despite its frequent use, previous studies have not yet demonstrated that the AOT is a valid measurement instrument to order individuals on actively open-minded thinking. High reliability values indicate that the observed AOT sumscores can classify individuals low to high. In addition, a positive correlation of observed sumscores with an external predictor shows that a high AOT sumscore is likely to go together with a high score on a relevant other variable. When an AOT sumscore is computed and used in analyses, the implicit assumption is that all items load on the same latent trait representing active open mindedness. Both reliability and correlational analyses, however, do not answer the question of whether the items used (i.e., the specific AOT sumscore), measure a single latent trait. Psychometric validation of the AOT scale, therefore, requires testing this assumption by assessing whether the responses of individuals to each item can be described as a function of a single latent trait (i.e., internal validity).

Svedholm-Häkkinen and Lindeman (2018) tested this assumption; however, their FAs suggested that the items they included did not reflect a unidimensional trait (i.e., no higher-order factor). Hence, a sumscore of their 17-item solution cannot be used to validly order individuals on the latent trait of actively open-minded thinking either, as it is unclear what concept a sumscore on this abbreviated version reflects. To illustrate our point, imagine that we measured four traits with a questionnaire: social economic status (SES) with 4 items, work satisfaction with 4 items, motivation to eat healthy with 4 items, and engagement in politics with 5 items. If we validly measured the four traits and subjected all 17 items to a FA, one would expect to find four intercorrelated factors without a higher-order factor (i.e., similar to the factor structure as Svedholm-Häkkinen \& Lindeman, 2018). However, even if the reliability was sufficient, a sumscore of those 17 items cannot be interpreted meaningfully, because a sum of a person's SES and motivation to eat healthy cannot easily be interpreted as a character trait of a person (i.e., the scales do not form a higher-order unidimensional trait). Also if this sumscore would correlate with other variables (e.g., mental health or having debts), this still does not allow for interpreting the sumscore as a single latent trait.

The aim of this study was to re-examine the validity of all 41 items of the AOT developed by Stanovich and West (2007), to see whether we could develop a valid shorter version that does reflect a (higher-order) unidimensional trait. To this end, we used Mokken scale analysis. Mokken scale analysis is a non-parametric item response theory approach that has advantages over the commonly conducted FAs, when analyzing data based on Likert type scales. An important advantage is that Mokken scale analysis is suitable for categorical data whereas FA requires data at the interval level. In the field of psychometrics it is argued that treating Likert scale data as interval data can be problematic (Liddell \& Kruschke, 2018). When treating Likert scale data instead as being ordinal, it is technically not possible to test for the normality assumption of FA because the difference between two successive values cannot be quantified. Even if one would still assess normality with ordinal data, Likert-scale items typically indicate skewed or polarized distributions (Jamieson, 2004; Liddell \& Kruschke, 2018). An additional advantage of Mokken scale analysis in this respect, is that it does not require multivariate normality or linear correlations between items. In the present study, we conducted an exploratory Mokken scale analysis (Mokken, 1971; Sijtsma \& Molenaar, 2002) 
on the Dutch version of the AOT (translated version used in Heijltjes et al., 2014) using two samples of higher education students $(\mathrm{N}=930$ and $\mathrm{N}=509)$ to see whether we could obtain a valid shorter version of the AOT. In addition, for comparability with prior work by Stanovich and West (2007) and by Svedholm-Häkkinen and Lindeman (2018) we conducted two CFAs representing their proposed models.

\subsection{METHOD}

All materials, datasets, R-code, and output are stored on an Open Science Framework (OSF) page for this project, see https://osf.io/4hxzu/.

\subsubsection{PARTICIPANTS AND PROCEDURE}

We obtained anonymized AOT datasets from a Dutch University of Applied Sciences ${ }^{4}$, where the AOT (see next section) was filled out on a computer by first year students, as part of a critical thinking course they were enrolled in. It was not possible to skip questions and only fully completed questionnaires could be submitted, so there was no missing data.

We repeated the same Mokken scale analyses on two datasets to see whether we obtained similar results. Dataset A $(\mathrm{N}=930)$ was a merged dataset that consisted of 460 students in the economics and business domain (data collected in 2014) and 470 students in the health care domain (data collected in 2016). Age and sex were indicated by 908 participants, whose mean age was 18.84 years $(\mathrm{SD}=2.30)$ and $55 \%$ of whom were female. Dataset $\mathrm{B}(\mathrm{N}=509)$ was a merged dataset that consisted of 257 students in the marketing and business management domain (data collected in 2017) and 252 students in the health care domain (data collected in 2017). Age and sex were indicated by 506 participants, whose mean age was 18.82 years (SD $=2.46$ ) and of whom $50 \%$ were female.

\subsubsection{ACTIVELY OPEN-MINDED THINKING SCALE}

We used a Dutch translation of the original 41-item version of the AOT (Stanovich \& West, 2007). In a previous study, Heijltjes et al. (2014) made the Dutch translation, which was checked by two persons one of whom was a native English speaker. In line with the original scale, the response format consisted of six answering categories: Strongly agree (6), Moderately agree (5), Slightly agree (4), Slightly disagree (3), Moderately disagree (2), and Strongly disagree (1). We reverse scored 30 items so that for all items a higher score indicated a stronger disposition towards actively open-minded thinking 5 (for all items, see OSF page).

\footnotetext{
${ }^{4}$ The Dutch education system distinguishes between higher professional education offered by universities of applied sciences (Bachelor, Master), and academic education offered by research universities (Bachelor, Master, PhD, with the $\mathrm{PhD}$ being an additional four-year trajectory after a Master degree).

${ }^{5}$ The students additionally completed a Dutch translation of 18-item (short form) Need for Cognition questionnaire (NFC; Cacioppo et al., 1984) that we intended to use as criterion variable to assess the validity of our newly obtained AOT in subsequent analyses. However, since our results did not yield a useful item selection for the AOT (see Results section), we did not use this variable in further analyses.
} 


\subsubsection{ANALYSES}

Mokken scale analyses. We conducted an exploratory Mokken scale analysis (Mokken, 1971; Molenaar \& Sijtsma, 2000) in order to abstract a valid shorter version of the AOT from the total item pool. In contrast to factor analysis, Mokken scale analysis requires only few assumptions and is, therefore, robust to problems concerning the distribution of the underlying data. First, the model underlying Mokken scale analysis assumes unidimensionality, which means that all items in a particular test measure the same latent trait (Sijtsma \& Molenaar, 2002). Second, the underlying model assumes local independence, which means that a person's response to an item is not influenced by his or her responses to the other items in the test, given the underlying latent trait. However, if students gain knowledge during the test, which they can use to answer the next items in the very same test, the assumption of local independence is violated. The third and final assumption is that the probability of answering the item correctly (or, in case of polytomous items, the probability of agreeing to the item) increases or stays the same as the ability level increases or, put more technically, that the response functions of the items (IRFs) are monotonically nondecreasing (Sijtsma \& Molenaar, 2002). Furthermore, Mokken scale analysis is suitable for the analysis of categorical data. The AOT items all have six ordered response categories, ranging from "disagree strongly" to "agree strongly". Inspecting the frequency distributions of the 41 AOT items indicated that for some items the distribution was skewed (as such, Mokken scale analysis was most suitable for our AOT data as it does not assume the data to be normally distributed).

Three scalability coefficients were used to determine whether or not items formed a scale, and as diagnostics to assess the strength of the scales (Kuijpers, 2015): (1) item-pair scalability coefficient $H_{i j}$, which expresses the strength of the association between items $i$ and $j$ given their marginal distributions; (2) item scalability coefficient $H_{j}$, which expresses how well item $j$ fits with the other items in a test, and also indicates the extent to which item $j$ discriminates between respondents (Sijtsma \& Molenaar, 2002, p. 66); and (3) total-scale scalability coefficient $H$, which expresses the degree to which respondents can be ordered by means of a set of items (Sijtsma \& Molenaar, 2002, pp. 36, 39). A set of items form a unidimensional scale if (1) all $H_{i j} \geq 0$ (i.e., the underlying model assumes positive inter-item covariances) and (2) if $H_{j}>\mathrm{c}>0$ for all $j$. The latter indicates that all item scalability coefficients should be at least positive, and rather above a positive value c (by default set to .3), such that nondiscriminating items or only weakly discriminating items are excluded from the scale. As follows from these two criteria, the value of the total-scale scalability coefficient $H$ should be at least .3 (Kuijpers et al., 2013; Mokken, 1971; Molenaar \& Sijtsma, 2000). $H$-values lower than .3 are regarded as indicating that the set of items is poorly scalable. The Mokken scale analysis was performed using the 'Mokken' package in R (R Development Core Team, 2008; Van der Ark, 2007).

Confirmatory factor analyses. For comparability with prior work by Stanovich and West (2007) and by Svedholm-Häkkinen and Lindeman (2018), we also ran two CFAs on dataset A and B . First, we ran a CFA on the model proposed by Stanovich and West (2007): a one factor with the 41 items as indicators of a single trait. Second, we ran a CFA based on the final model proposed by Svedholm-Häkkinen and Lindeman (2018): a 17-item version with four intercorrelated factors without one higher order factor. We used the 'Lavaan' package 
in R (R Development Core Team, 2008; Rosseel, 2012) with robust weighted least squares (WLSMV) as estimation method. This estimator is seen as most suitable for categorical data (Brown, 2006). To be fully consistent with Svedholm-Häkkinen and Lindeman (2018), we also ran the CFAs with ML estimation, yielding highly similar results (for these results, see OSF page).

\subsection{RESULTS}

\subsubsection{MOKKEN SCALE ANALYSES}

The Mokken scale analysis performed on the first dataset (A) showed that no subset of items could be constructed that validly measured a single latent trait. None of the 41 items could discriminate sufficiently between respondents with varying latent trait levels, all $H_{j} \mathrm{~s}$ $\leq .182, H=.105$ (for all $41 H_{j}$ s and the item-pair scalability coefficients, see OSF page). Furthermore, the explorative analyses indicated that 18 out 41 items formed eight separate scales with each two to three items at most. The remaining 23 items were left unscalable, that is, the items were not or even more poorly discriminating and/or covaried negatively with items included in one of the eight scales. Table 7.2 shows the eight scales and the item scalability coefficients $\left(H_{j}\right)$ with the corresponding standard errors for the 18 scalable items (for the item-pair scalability coefficients, see OSF page). Only two items had a $H_{j}$ that was significantly above .3 and none of the scales consisted entirely of items with $H_{j}$ significantly $>$.3. Due to the poor quality of the scales, total scalability coefficients for the separate scales were not calculated. In addition to the finding that the scales did not discriminate between respondents, Cronbach's alpha was low for the two scales consisting of three items (for two item-scales, alpha cannot be computed), $\mathrm{C} 1: \alpha=.58 ; \mathrm{C} 2: \alpha=.55$.

Findings for dataset B were more or less similar. Again, none of the 41 items could discriminate sufficiently between respondents with varying latent trait levels, all $H_{s} \leq$ $.195, H=.100$ (for all $41 H_{s}$ and the item-pair scalability coefficients, see OSF page). The explorative analyses identified seven separate scales with five items in the first scale and two items in each of the other six scales. The 24 remaining items were left unscalable. Table 7.3 shows the seven scales and the item scalability coefficients $\left(H_{j}\right)$ with the corresponding standard errors for the 17 scalable items (for the item-pair scalability coefficients, see OSF page). Again, only two items had a $H_{j}$ that was significantly above .3 and none of the scales consisted entirely of items with $H_{j}$ significantly $>.3$. Due to the poor quality of the scales, total scalability coefficients of the separate scales were not calculated. Finally, the one scale including more than two items was marginally reliable, $\mathrm{C} 1: \alpha=.67$.

Thus, the results of the Mokken scale analyses on both datasets A and B suggested that the AOT was not measuring a unidimensional latent trait. Furthermore, the item scales found did not have sufficient discriminative power and had a poor reliability and could therefore not be considered to be useful (sub)scales. In addition, it has been argued that using many subscales with only two or three items can have a negative impact on the reliability, validity and measurement precision of a scale (Kruyen et al., 2012, 2013; Mellenbergh, 1996; Reise \& Waller, 2009). 
Table 7.2. Clusters, item scalability coefficients $\left(H_{\mathrm{j}}\right)$, and the corresponding standard errors for the 18 scalable items selected by the automated selection procedure on Dataset A ( $N$ $=930)$

\begin{tabular}{|c|c|c|c|c|c|c|}
\hline & Item & Cluster & $\mathrm{M}^{\mathrm{a}}$ & SD & $H_{j}$ & SE \\
\hline 5. & $\begin{array}{l}\text { There are two kinds of people in this world: } \\
\text { those who are for the truth and those who are } \\
\text { against the truth. (R) }\end{array}$ & C1 & 4.10 & 1.30 & $0.376^{*}$ & 0.029 \\
\hline 8. & $\begin{array}{l}\text { I think there are many wrong ways, but only } \\
\text { one right way, to almost anything. }(R)\end{array}$ & C1 & 5.00 & 0.98 & 0.325 & 0.032 \\
\hline 17. & $\begin{array}{l}\text { There are basically two, kinds of people in } \\
\text { this world, good and bad. (R) }\end{array}$ & C1 & 4.63 & 1.34 & $0.369^{*}$ & 0.028 \\
\hline 4. & $\begin{array}{l}\text { A person should always consider new } \\
\text { possibilities. }\end{array}$ & C2 & 5.01 & 0.78 & 0.310 & 0.034 \\
\hline 37. & $\begin{array}{l}\text { Beliefs should always be revised in response } \\
\text { to new information or evidence }\end{array}$ & C2 & 4.50 & 0.85 & 0.351 & 0.032 \\
\hline 41. & $\begin{array}{l}\text { People should always take into consideration } \\
\text { evidence that goes against their beliefs. }\end{array}$ & C2 & 4.65 & 0.89 & 0.350 & 0.034 \\
\hline 15. & $\begin{array}{l}\text { It is important to persevere in your beliefs } \\
\text { even when evidence is brought to bear } \\
\text { against them. }(R)\end{array}$ & C3 & 3.90 & 1.22 & 0.369 & 0.036 \\
\hline 19. & $\begin{array}{l}\text { Certain beliefs are just too important to } \\
\text { abandon no matter how good case can be } \\
\text { made against them. (R) }\end{array}$ & C3 & 3.11 & 1.13 & 0.369 & 0.036 \\
\hline 25. & $\begin{array}{l}\text { My beliefs would not have been very different } \\
\text { if I had been raised by a different set of } \\
\text { parents. }(R)\end{array}$ & C4 & 3.89 & 1.33 & 0.356 & 0.037 \\
\hline 28. & $\begin{array}{l}\text { Even if my environment (family, } \\
\text { neighborhood, schools) had been different, I } \\
\text { probably would have the same religious } \\
\text { views. (R) }\end{array}$ & C4 & 2.85 & 1.38 & 0.356 & 0.037 \\
\hline 23. & $\begin{array}{l}\text { I believe that loyalty to one's ideals and } \\
\text { principles is more important than "open- } \\
\text { mindedness". (R) }\end{array}$ & C5 & 4.46 & 0.97 & 0.347 & 0.039 \\
\hline 33. & $\begin{array}{l}\text { One should disregard evidence that conflicts } \\
\text { with your established beliefs. }(R)\end{array}$ & C5 & 4.82 & 0.89 & 0.347 & 0.039 \\
\hline 11. & $\begin{array}{l}\text { There are a number of people I have come to } \\
\text { hate because of the things they stand for. }(R)\end{array}$ & C6 & 4.10 & 1.34 & 0.345 & 0.031 \\
\hline 31. & $\begin{array}{l}\text { My blood boils over whenever a person } \\
\text { stubbornly refuses to admit he's wrong. (R) }\end{array}$ & C6 & 2.92 & 1.30 & 0.345 & 0.031 \\
\hline 13. & $\begin{array}{l}\text { No one can talk me out of something I know } \\
\text { is right. }(R)\end{array}$ & $\mathrm{C} 7$ & 3.00 & 1.21 & 0.335 & 0.036 \\
\hline 14. & $\begin{array}{l}\text { Basically, I know everything I need to know } \\
\text { about the important things in life. }(R)\end{array}$ & C7 & 4.32 & 1.17 & 0.335 & 0.036 \\
\hline 16. & $\begin{array}{l}\text { Considering too many different opinions often } \\
\text { leads to bad decisions. }(R)\end{array}$ & C8 & 3.93 & 1.16 & 0.331 & 0.036 \\
\hline 35. & $\begin{array}{l}\text { A group which tolerates too much difference } \\
\text { of opinion among its members cannot exist } \\
\text { for long. }(\mathrm{R})\end{array}$ & C8 & 4.06 & 1.21 & 0.331 & 0.036 \\
\hline
\end{tabular}

Note. $\mathrm{R}=$ reverse scored item. ${ }^{*} H_{j}$ significantly above. 3 with $p<.05 .{ }^{a}$ higher $=$ more actively openminded thinking. 
Table 7.3. Clusters, item scalability coefficients $\left(H_{\mathrm{j}}\right)$, and the corresponding standard errors for the 17 scalable items selected by the automated selection procedure on Dataset $B(N$ $=509$ )

\begin{tabular}{|c|c|c|c|c|c|c|}
\hline Item & & Cluster & $\mathrm{M}^{\mathrm{a}}$ & SD & $H_{j}$ & SE \\
\hline 5. & $\begin{array}{l}\text { There are two kinds of people in this world: } \\
\text { those who are for the truth and those who are } \\
\text { against the truth. }(\mathrm{R})\end{array}$ & $\mathrm{C} 1$ & 3.93 & 1.30 & 0.315 & 0.034 \\
\hline 8. & $\begin{array}{l}\text { I think there are many wrong ways, but only } \\
\text { one right way, to almost anything. (R) }\end{array}$ & $\mathrm{C} 1$ & 5.02 & 0.86 & $0.373^{*}$ & 0.031 \\
\hline 17. & $\begin{array}{l}\text { There are basically two, kinds of people in } \\
\text { this world, good and bad. (R) }\end{array}$ & C1 & 4.49 & 1.38 & $0.361^{*}$ & 0.030 \\
\hline 24. & $\begin{array}{l}\text { Of all the different philosophies which exist in } \\
\text { the world there is probably only one which is } \\
\text { correct. }(\mathrm{R})\end{array}$ & $\mathrm{C} 1$ & 5.12 & 0.89 & 0.316 & 0.034 \\
\hline 33. & $\begin{array}{l}\text { One should disregard evidence that conflicts } \\
\text { with your established beliefs. }(R)\end{array}$ & $\mathrm{C} 1$ & 4.73 & 0.92 & 0.309 & 0.034 \\
\hline 6. & $\begin{array}{l}\text { Changing your mind is a sign of weakness. } \\
\text { (R) }\end{array}$ & C2 & 4.96 & 1.03 & 0.357 & 0.051 \\
\hline 38. & $\begin{array}{l}\text { I think that if people don't know what they } \\
\text { believe in by the time they're } 25 \text {, there's } \\
\text { something wrong with them. (R) }\end{array}$ & $\mathrm{C} 2$ & 4.42 & 1.21 & 0.357 & 0.051 \\
\hline 25. & $\begin{array}{l}\text { My beliefs would not have been very different } \\
\text { if I had been raised by a different set of } \\
\text { parents. (R) }\end{array}$ & $\mathrm{C} 3$ & 3.88 & 1.29 & 0.350 & 0.049 \\
\hline 28. & $\begin{array}{l}\text { Even if my environment (family, } \\
\text { neighborhood, schools) had been different, I } \\
\text { probably would have the same religious } \\
\text { views. (R) }\end{array}$ & C3 & 2.99 & 1.43 & 0.350 & 0.049 \\
\hline 37. & $\begin{array}{l}\text { Beliefs should always be revised in response } \\
\text { to new information or evidence. }\end{array}$ & C4 & 4.47 & 0.87 & 0.338 & 0.046 \\
\hline 41. & $\begin{array}{l}\text { People should always take into consideration } \\
\text { evidence that goes against their beliefs. }\end{array}$ & C4 & 4.58 & 0.91 & 0.338 & 0.046 \\
\hline 21. & $\begin{array}{l}\text { It is a noble thing when someone holds the } \\
\text { same beliefs as their parents. }(R)\end{array}$ & C5 & 4.00 & 1.05 & 0.312 & 0.049 \\
\hline 22. & $\begin{array}{l}\text { Coming to decisions quickly is a sign of } \\
\text { wisdom. }(R)\end{array}$ & C5 & 4.33 & 1.12 & 0.312 & 0.049 \\
\hline 13. & $\begin{array}{l}\text { No one can talk me out of something I know } \\
\text { is right. (R) }\end{array}$ & C6 & 2.99 & 1.21 & 0.312 & 0.049 \\
\hline 14. & $\begin{array}{l}\text { Basically, I know everything I need to know } \\
\text { about the important things in life. }(R)\end{array}$ & C6 & 4.33 & 1.14 & 0.312 & 0.049 \\
\hline 35. & $\begin{array}{l}\text { A group which tolerates too much difference } \\
\text { of opinion among its members cannot exist } \\
\text { for long. }(R)\end{array}$ & $\mathrm{C7}$ & 4.08 & 1.18 & 0.300 & 0.048 \\
\hline 39. & $\begin{array}{l}\text { I believe letting students hear controversial } \\
\text { speakers can only confuse and mislead them. } \\
\text { (R) }\end{array}$ & $\mathrm{C} 7$ & 4.22 & 1.01 & 0.300 & 0.048 \\
\hline
\end{tabular}

Note. $\mathrm{R}=$ reverse scored item. ${ }^{*} H_{j}$ significantly above. 3 with $p<.05 .{ }^{a}$ higher $=$ more actively openminded thinking. 


\subsubsection{CONFIRMATORY FACTOR ANALYSES}

We first conducted a CFA on dataset A and B, testing the one-factor model on all 41 items as proposed by Stanovich and West (2007). For dataset A, we obtained mixed results on the model fit indices. For the absolute fit indices, Chi-square indicated a poor fit, $\chi^{2}(779)=$ 2000.67, $p<.001$, which could be expected given the large sample size. Root Mean Square Error of Approximation (RMSEA) and Standardized Root Mean Square Residual (SRMR), on the other hand, showed an acceptable fit, indicating that there was an acceptable discrepancy between hypothesized model (with optimal parameter estimates) and the actually obtained sample data (covariance matrix), RMSEA $=0.041, p=.999 ; \mathrm{SRMR}=0.06$. The incremental fit indices (analogous to $\mathrm{R}^{2}$ ), however, showed poor fit, indicating that the improved data fit by the tested one-factor model was only marginal when compared to the data fit of the null model (in which all the observed variable are uncorrelated), Comparative Fit Index (CFI) $=0.69$; Tucker Lewis Index (TLI) $=0.67$. Hu and Bentler (1999) argue that values close to .95 for CFI and TLI in combination with values close to .06 and .08 for RMSEA and SRMR, respectively, are needed to conclude that there is a relatively good fit between the hypothesized model and the observed data. Following their guidelines, we concluded that the model did not describe the data adequately. Furthermore, 27 out of 41 items had small standardized factor loadings ( $<.4$; for all factor loadings, see OSF page), indicating that those items could not discriminate between respondents with varying trait levels (Brown, 2006). In line with Stanovich and West (2007), the scale as a whole was reliable, $\alpha=.81$. We obtained more or less similar results for dataset $\mathrm{B}, \chi^{2}(779)=1384.80, p<.001$; RMSEA $=.039, p=$ $.990 ; \mathrm{SRMR}=.064 ; \mathrm{CFI}=0.71$; TLI $=0.70$. Also, 27 items had small standardized factor loadings $(<.4)$ and the Cronbach's alpha for the total scale was .80 .

Next, we conducted a CFA on dataset A and B, testing the intercorrelated four-factor model without a higher-order factor on the 17-item AOT as proposed by Svedholm-Häkkinen $\&$ Lindeman (2018). Both for dataset A and B, we obtained mixed results. For dataset A, the absolute fit indices indicated an acceptable fit, $\chi^{2}(113)=431.39, p<.001$; $\operatorname{RMSEA}=0.055$, $p=0.06$; SRMR $=0.058$, whereas the incremental fit indices indicated a poor data fit; $\mathrm{CFI}=$ 0.75 ; TLI $=0.70$. Hence, this model also did not describe the data adequately. Additionally, eight out of 17 items had small standardized factor loadings $(<.4$; for all factor loadings, see OSF page). The scale as a whole had a Cronbach's alpha of .67 , and the subscales Dogmatism, Fact Resistance, Liberalism, and Belief Personification had alphas of .53, .56, .32 and .51 respectively. For dataset $B$, we obtained rather similar results, $\chi^{2}(113)=367.07$, $p<.001 ; \mathrm{RMSEA}=.067, p<.001 ; \mathrm{SRMR}=.069 ; \mathrm{CFI}=0.67 ; \mathrm{TLI}=0.60$. Eight items had small standardized factor loadings $(<.4)$ and the Cronbach's alpha for the total scale was .66. The subscale alphas were $.60, .51, .24, .46$, for Dogmatism, Fact Resistance, Liberalism, and Belief personification, respectively.

In sum, both the Mokken scale analyses and the one-factor CFA suggested that AOT was not measuring a unidimensional trait and did not yield an item set that could be used 
to validly order individuals on the single trait actively open minded thinking. Also, both the Mokken scale analyses and the four-factor CFA did not yield reliable subscales that could discriminate between respondents.

\subsection{DISCUSSION}

The aim of this study was to obtain a valid shorter version of the AOT developed by Stanovich and West (2007) that could be used to order individuals on the latent trait actively open-minded thinking. Our results did not provide support for the hypothesis that either the 41-item AOT or a subset of items would measure actively open-minded thinking as a single latent trait. The Mokken scale analyses performed on two large datasets of Dutch first-year higher professional education students showed that none of the items discriminated very well between students on the (assumed) latent trait. In addition, no adequate AOT subscales could be identified. These findings imply that - for the studied population - sumscores on the AOT do not provide insight into the concept it aims to measure.

\subsubsection{RELATING THE CURRENT RESULTS TO PREVIOUS FINDINGS}

Sumscores on the AOT are widely used in, for example, correlational analyses. When one computes a sumscore and assumes that it provides insights into the construct "actively open-minded thinking", one assumes that all items load on the same assumed latent trait. However, the evidence so far, including our results, do not support this assumption. The results of our Mokken scale analyses, our one-factor CFA, and the results of Svedholm-Häkkinen and Lindeman (2018) indicated that the AOT is not measuring one unitary trait. This renders the reported reliabilities for the scale as a whole (see Table 7.1) rather meaningless, as Cronbach's alpha assumes a unidimensional construct.

In addition to the fact that the scale does not measure a unidimensional trait, we also found no evidence for meaningful subscales. Here, our results differ somewhat from Svedholm-Häkkinen and Lindeman (2018), who found that a 17-item version of the AOT measured four separate subscales. Our Mokken scale analyses indicated that more than half of the 41 items were left unscalable (i.e., could not be included in a subscale) and that none of the (very small) subscales that were formed had sufficient discriminative power. Hence, the items included in a subscale could not order participants with varying levels of the latent trait that the scale was potentially measuring. Furthermore, the formed subscales were not reliable. Our CFA testing the four-factor model (without one higher-order factor) proposed by Svedholm-Häkkinen and Lindeman (2018) did not describe the data adequately. We obtained acceptable values for the absolute fit indices, but a poor values for the incremental fit indices. Hence, the tested four-factor model fitted acceptably with the obtained sample data but the model fitted the data only slightly better than the worst possible model would 
do. Furthermore, 8 of the 17 items had low factor loadings and could thus not discriminate between participants. Also note that both in our study and in the study by Svedholm-Häkkinen and Lindeman (2018) low reliabilities for the four subscales were obtained. Hence, there is still no convincing evidence that scores on the subscales can be interpreted meaningfully. One possible explanation for the divergent results with Svedholm-Häkkinen and Lindeman (2018) regarding the CFAs may be that, the Likert type AOT items are not suitable for FA and therefore do not yield robust results across studies (Magidson \& Vermunt, 2003). A more likely explanation, however seems that the AOT items do not sufficiently measure what they intend to. Taking the Mokken scale and FAs together, it seems that the AOT items included in the available studies so far are not measuring a single psychological trait actively open-minded thinking nor any subtraits. The construct validity and content validity of the items should be improved in order to obtain a valid measurement instrument.

If it is unclear what the sumscore on the AOT represents, it is also unclear how the correlations that previous studies found between the AOT and other variables (such as other dispositions like the tendency to enjoy and engage in thinking, measured with the NFC, or performance on critical thinking tests, see e.g., Heijltjes et al., 2014; Svedholm-Häkkinen \& Lindeman, 2018; Toplak et al. 2014a) should be interpreted. The correlations between the AOT and other thinking dispositions may mean that some AOT items measure more or less the same as some items from other disposition questionnaires, and that the AOT sumscores therefore correlate with these variables (e.g., an item in the AOT is 'If I think longer about a problem I will be more likely to solve it' and an item in the Need for Cognition scale is 'I would prefer complex to simple problems'). It may also be that both the AOT and its criterion variables (e.g., rational reasoning) implicitly measure something else that we are not aware of and that this causes a correlation (cf. the third variable problem).

\subsubsection{LIMITATIONS AND FURTHER RESEARCH}

To our knowledge, this is the first study that investigated the psychometric properties of the AOT using Mokken scale analysis, which can be considered to be more suitable than the more commonly used FAs because it is suitable for the categorical responses to the AOT items and robust to violations of multivariate normality and linear correlation between items (Flora et al., 2012; Jamieson, 2004; Liddell \& Kruschke, 2018; Mokken, 1971; Sijtsma \& Molenaar, 2002). Nevertheless, two limitations of our study should be noted. First, it is possible that all the participants in our study sample were very strong actively open-minded thinkers (i.e., relatively high average item scores items and therefore quite homogeneous), resulting in little or no variance in item scores. Participants in our sample, however, had a rather similar total score on the 41-item AOT $(\mathrm{M}=171.8, \mathrm{SD}=15.2)$ compared to the Stanovich and West study (2007) that introduced this version of the AOT $(\mathrm{M}=170.7, \mathrm{SD}=18.2)$. A second limitation is that our analyses were conducted on the Dutch version of the AOT. Although we see no obvious reason to expect diverting results, it would be interesting to replicate our Mokken scale analyses with other datasets on the English version of the AOT. 


\subsubsection{CONCLUSION}

To conclude, the results of our study suggest that there is no item set of the 41 item version of the AOT that can be used to validly order individuals on their ability to think active open-mindedly, which is a crucial assumption when using it in research. Consequently, it is questionable whether scores on the AOT provide insights into the concept it aims to measure. If the present Mokken scale analyses were replicated with English AOT data, this would be a strong argument for starting the process of (re-)designing a new scale to measure actively open-minded thinking or to consider alternative measures of thinking dispositions. 
Chapter 8

Summary and discussion 
Teachers play a crucial role in attaining a major objective of higher education: fostering students' critical thinking (CT). Yet, little is known about how to support teachers in fulfilling this role successfully. Therefore, the main aim of this dissertation was to start investigating how to equip higher education teachers with the knowledge and skills necessary for teaching one essential CT-skill: the ability to avoid bias in reasoning and decision-making (West et al., 2008). For being able to teach CT, there are three important variables, which formed the central focus in this dissertation: (1) teachers' own CT-skills, operationalized as ability to avoid bias in one's own reasoning and decision-making; (2) teachers' CT-teaching-skills, operationalized as ability to detect and explain biases in students' reasoning; and (3) teachers' positive attitudes towards teaching $\mathrm{CT}$, operationalized as perceived relevance of and perceived competence in teaching CT. The first part of this dissertation (Chapters 2, 3, and 4) addressed the main question "How can higher education teachers be equipped with the knowledge and skills necessary for teaching critical thinking?'. The second part (Chapters 5, 6, and 7) contained three additional studies on cognitive mechanisms (Chapters 5 and 6) and dispositions (Chapter 7) that underlie biased reasoning. In this Chapter, I will first summarize the main findings, then discuss them in light of scientific implications, practical implications, and future directions. Thereafter, I point out the overarching limitations of all studies and, finally, I conclude with a recommendation for the educational practice.

\section{PART 1: TEACHING CRITICAL THINKING}

\subsubsection{SUMMARY}

The study in Chapter 2 investigated what teacher characteristics are associated with higher education teachers' CT-skills and their attitudes towards teaching CT. CT-skills were assessed with the Cognitive Reflection Test (CRT), which measures the ability to avoid biased reasoning on seven short math problems. Teaching attitudes were measured with a questionnaire that addressed teachers' perceived relevance of and perceived competence in teaching CT. Results of a structural equation model $(N=263)$ showed that a stronger disposition towards effortful thinking, teaching in a more technological domain, and a higher level of education were related to better performance on the CRT. Stronger thinking dispositions were also positively related to teachers' perceived relevance of teaching CT, yet a better CRT performance was not. Finally, confidence in CRT performance rather than actual test performance was related to higher perceived competence in teaching CT.

The quasi-experimental study $(N=54)$ in Chapter 3 tested whether a three-session teacher training on CT positively affected their CT-skills, CT-teaching-skills, and attitudes towards teaching CT, compared to an untreated control condition. The first session provided 
instructions on reasoning biases and how to avoid them, followed by practice tasks. The second and third sessions focused on strengthening teachers' attitudes and skills towards teaching CT through discussing the relevance of teaching CT, providing extra opportunity for practice, designing a domain-specific CT-task, and discussing ways to integrate CT during teaching. Overall, the training had a large effect on teachers' ability to avoid the instructed biases on learning tasks. However, it did not affect their ability to avoid non-instructed biases on transfer tasks, although the trained teachers did invest more effort in solving the transfer tasks than non-trained teachers. This latter finding may indicate that trained teachers were aware that their answers could be incorrect and engaged in deeper thinking about the tasks yet without being able to come up with the correct answer. With regard to CT-teaching-skills, teachers' ability to detect biases hidden in excerpts from student papers strongly improved after training; however, they still had difficulties in clearly explaining why a student's reasoning was biased. On average, more than half of the detected biases were still explained incorrectly. Finally, the training did not positively affect teachers' attitudes towards teaching CT. Perceived relevance was already high prior to the training in both the training and control condition. Perceived competence in teaching CT decreased temporarily in the training condition after the first training session, but this negative effect disappeared after the final (third) session.

The experiment described in Chapter $4(N=37)$ focused on the crucial CT-teaching-skill of providing feedback on students' reasoning. It was investigated whether the ability to explain biases in students' reasoning would improve from training. Teachers in the control condition participated in a CT-training session on avoiding biases in their own reasoning (cf. first training session in Chapter 3) and teachers in the experimental condition additionally followed an individual online training on how to clearly explain biases in students' written arguments. At the immediate posttest, the teachers in the experimental condition improved explaining biases they had practiced with (learning), whereas teachers in the control condition did not. This improvement was maintained at delayed posttest two weeks later, but surprisingly - teachers in the control condition had also improved. Both conditions improved (slightly and equally) explaining non-practiced biases (transfer). Perceived relevance of and perceived competence in teaching CT was relatively high in both conditions and constant across test occasions.

\subsubsection{DISCUSSION: IMPLICATIONS AND FUTURE DIRECTIONS.}

The studies presented in Chapters 2, 3, and 4 break new ground by exploring teachers' CT and their teaching of CT. Together, the results reported in those chapters give some first answers to the question of how to equip higher education teachers with the knowledge and skills necessary for teaching CT, and specifically, for teaching to avoid biased reasoning.

Teachers' critical thinking. First, the results add to research in the reasoning and decision-making field, which has shown that people's thinking dispositions, mindware (i.e., knowledge and skills needed for the reasoning situation), and cognitive ability are important predictors of the ability to avoid biased reasoning (Campitelli \& Gerrans, 2014; 
Frederick, 2005; Heijltjes et al., 2015; Klaczynski, 2014; Stanovich et al., 2016; West et al., 2008). Findings in Chapter 2 suggest that these theoretical variables can also be practically applied to teacher characteristics to predict their ability to avoid biased reasoning. Teachers' dispositions towards effortful thinking, their teaching domain, and level of education all explained unique variance in their CRT performance. Note that I view level of education as proxy of cognitive ability and teaching domain as proxy of requisite mindware for the CRT, which draws strongly on numerical abilities. Chapter 3 and 4 additionally indicate that mindware not only has a correlational effect on teachers' ability to avoid bias, but also a causal effect. Training teachers' mindware on how to avoid biases or on how to explain them adequately (through explicit instruction and practice opportunities) improved their performance on heuristic-and-biases tasks and their explanations of biases in students' reasoning.

Thus, the results presented in this dissertation not only extend prior findings on variables that might affect biased thinking to teachers, but they also have implications for future research in this area and for training teachers in educational practice. For instance, teaching domain and teachers' thinking dispositions may need to be taken into account when training teachers to improve CT-skills (e.g., considering questions like: What mindware is already available? What are their knowledge gaps? How disposed are the teachers towards [teaching] CT?). Future research in this area could address whether teaching domain would also be a predictor of the ability to explain biases in students' reasoning (next to being a predictor of the ability to avoid bias in teachers' own reasoning). Other interesting questions are whether thinking dispositions would moderate the effectiveness of training (see e.g., previous research with students, Heijltjes, Van Gog, Leppink, et al., 2014; Klaczynski, 2014), whether thinking dispositions could also be trained, and if so, whether a positively changed thinking disposition would lead to better performance on CT(-teaching)-tasks.

Training teachers' critical thinking. Next to adding to the literature in the reasoning and decision-making field, this dissertation's results also replicate and extend research on teaching CT to students. Previous experiments with higher education students showed that explicit instruction of cognitive biases (through a video or a text) combined with task practice improved their performance on learning tasks but not on transfer tasks (Heijltjes et al., 2015; Heijltjes, Van Gog, Leppink, et al., 2014; Heijltjes, Van Gog, \& Paas, 2014). In line with this, the current studies with higher education teachers show that explicit instruction and practice on how to avoid biases (Chapter 3) and on how to explain biases (Chapter 4) has a positive effect on teachers' performance on learning tasks, but not on transfer tasks. Although the two types of training did not affect transfer across CT-tasks, the training studied in Chapter 3 did affect transfer across contexts: instruction on how to avoid bias on heuristics-and-biases tasks had a large effect on teachers' ability to detect those biases in excerpts from student papers. However, effects on teachers' ability to explain these detected biases were small.

Thus, Chapter 3 and for 4 imply that teachers' CT-skills and CT-teaching-skills can be trained and, importantly, need to be trained. Especially the CT-teaching skill of explaining biases in students' reasoning is of crucial importance; we cannot expect students to improve their reasoning without providing them with adequate feedback on what is wrong in their 
reasoning and why, and what the correct line of reasoning would be. Nevertheless, several important questions for future research still remain. First, how can we support transfer to other CT-tasks? This is a very important question, because we cannot train every type of bias teachers will ever encounter, so teachers need to be able to apply the instructed principles to novel tasks (with similar underlying principles) and the ultimate goal is that they can also apply them to novel reasoning situations outside the training setting (i.e., in the classroom). I suspect that answering this transfer question will be a major challenge, as instructional methods that typically affect transfer of cognitive skills do not seem to affect transfer of CT-skills. For example, the studied teacher trainings in this dissertation relied on example-based learning (Van Gog et al., 2019), used generative learning activities (Fiorella \& Mayer, 2016), and applied the fading guidance principle (Renkl \& Atkinson, 2003) - all transfer promoting interventions - but did not support transfer across CT-tasks. Moreover, the research conducted in the other subproject within this larger research project (see Chapter 1) tested effects of other generative learning activities that typically foster transfer (e.g., self-explaining, interleaved practice, comparing correct and erroneous examples, repeated retrieval practice), yet, none of these instructional interventions yielded clear transfer effects across CT-tasks. Another open question is whether teacher CT-training would have sustained effects and how training would transfer to the actual teaching context. The ultimate question to be addressed in future research, is how students' CT-skills are affected by improved CT-teaching-skills of their teachers. Also, it is of practical importance to test the effectiveness of this type of training in different teaching domains and with other biases. Although the cognitive biases addressed in this dissertation are common in a wide range of situations (Pennycook et al., 2015b), there is an endless list of cognitive biases people can fall prey to, and it might differ per teaching domain which biases are most important to avoid. Especially given the difficulty of achieving transfer, practical implementation of teacher training requires knowledge on what particular reasoning biases are most important for teachers to be trained in, hereby distinguishing between biases relevant for each teaching domain and domain-specific biases (for a discussion on how to institutionally embed CT in higher education, see Elen et al., 2019).

Attitudes towards teaching critical thinking. Finally, despite the promising findings on the trainability of required CT(-teaching)-skills, the studies in all three chapters also indicate that improving these skills is by no means a guarantee that teachers' attitudes towards teaching CT will increase along with this. Confidence in one's CRT performance rather than actual performance appeared to predict teachers' perceived competence in teaching CT (Chapter 2) and, despite the fact that teachers' CT-teaching-skills increased after a CT-training, their perceived relevance of and perceived competence in teaching CT did not (Chapter 3 and 4). This could be problematic because previous research demonstrated that a positive attitude towards the relevance of teaching as subject and confidence in their ability to teach it are important predictors of effective teaching (Klassen \& Tze, 2014; Lorencová et al., 2019; Paul et al., 1997; Van Aalderen-Smeets \& Walma van der Molen, 2013, 2015). Note, however, that in all three studies teachers generally agreed with the relevance of teaching CT already prior to the study and, also, the average perceived competence in teaching CT was already 
moderately high. Whether or not that is sufficient is hard to say as we cannot quantify how relevant teachers should perceive the teaching of CT to be or how competent they should feel. Besides, it is possible that the teachers in my studies may have been more positive about CT compared to the average (inter)national higher education teacher, because they all worked at university of applied sciences that pays a lot of attention to the topic of CT. Yet, other international studies have also found that teachers perceive teaching CT as highly relevant (though they differ in views on what CT is and how to teach it; Choy \& Cheah, 2009; Dumitru, 2018; Morais et al., 2018; Paul et al., 1997; Stedman \& Adams, 2012). These results are still hard to compare directly as all studies relied on very different research methods and on small samples mostly. It would therefore be interesting for future research to investigate teachers' attitudes towards teaching CT in a large representative international sample.

Thus, the current results give no direct answer to the question on if and how teachers' attitudes towards teaching CT can be fostered. The findings seem to imply that changing teaching attitudes may either require more training time and explicit attention, or more sensitive instruments to measure attitudes than those used in the current studies. Hence, next to investigating how to train teachers' CT-skills, it crucial to start investigating how to implement teacher training on CT in such a way that teachers feel competent enough to make CT an integrated part of their teaching practice. Note, however, that not only the degree of perceived competence, but also the degree to which this perception matches with actual competence (i.e., calibration) is important. For instance, a teacher with a very high perceived competence but low actual ability in CT-teaching (overconfidence), may teach CT explicitly to students, but not necessarily in an effective way.

\section{PART 2: COGNITIVE MECHANISMS AND DISPOSITIONS UNDERLYING BIASED REASONING}

\subsubsection{SUMMARY}

The studies in Part 2 of this dissertation focused on cognitive mechanisms and dispositions underlying biased reasoning. Chapter 5 built on recent research in which it has been shown that most biased reasoners seem somehow aware that their reasoning is incorrect; they show robust signs of error or "conflict" detection (De Neys \& Glumicic, 2008; Frey et al., 2018; Pennycook et al., 2015a). One important shortcoming in this research, however, is that the conflict detection effect was studied with classical, arguably non-realistic, heuristics-and-biases tasks. Therefore, the present study $(N=159)$ investigated whether conflict detection also occurs during reasoning on longer, more complex, and more realistic tasks involving decision-making (i.e., avoiding bias in own reasoning on problem-solving tasks) or decision-evaluation (recognizing bias in reasoning of others on vignette tasks; paralleling a teacher's task to evaluate students' reasoning in assignments). In line with previous studies, conflict detection was measured by comparing participants' confidence ratings and response times on so-called conflict tasks and on no-conflict tasks. Conflict tasks are designed to prime a heuristic or intuitive response that conflicts with more logical considerations. No-conflict tasks, on the other hand, prime the exact same heuristic response 
but here this heuristic response is also the correct response (i.e., no conflict between the heuristic and logical considerations; see Chapter 5 for examples). If biased reasoners do not notice the logical conflict in conflict tasks, their confidence and response times on (incorrect) heuristic responses to conflict tasks should not differ from their confidence and response times on (correct) heuristic responses to no-conflict tasks. However, if they show decreased confidence or longer response times on their (incorrect) heuristic responses to conflict tasks relative to (correct) heuristic responses to no-conflict tasks, they show some signs of sensitivity to the fact that their heuristic response may not be correct. Results showed that conflict detection (as indicated by decreased confidence) occurred during reasoning on the more realistic tasks used in the present study and that the conflict detection effect was very similar during reasoning on decision-making and decision-evaluation tasks. Also, conflict detectors showing a larger conflict detection effect (i.e., larger difference between confidence on conflict and no-conflict tasks) were more likely to start reasoning correctly on next conflict problems instead of remaining biased on all problems. Response time indices appeared to be less reliable measures of conflict detection on the studied tasks. This study provided the first tentative evidence that the conflict detection mechanism may also apply to biased decision-making and decision-evaluation on real-world scenarios. These findings are especially relevant for (studying) reasoning in contexts in which recognizing reasoning errors is important (e.g., in medicine where doctors have to evaluate initial diagnoses of others, or in education where teachers have to detect and give feedback on biases in their students' reasoning).

The study in Chapter 6 explored people's reasoning on the popular bat-and-ball problem (one of the CRT items used in Chapter 2). The aim was to investigate the impact of minimal response feedback (i.e., telling participants whether their response is correct or incorrect; frequently done in educational settings) on people's task performance and conflict detection. To examine the nature of a potential learning effect, a two-response paradigm was adopted, in which participants first had to indicate their initial, intuitive hunch and then could take time to deliberate and change their answer. Results of two experiments $(N=112$ and $N=$ 80) clearly indicated that feedback had, on average, no significant effect on participants' accuracy on (variations of) this problem. Explorative analyses did reveal an increased conflict detection effect (indexed by response time) after receiving feedback on errors, suggesting that participants did process the feedback but that the additional deliberation time did not help most participants to arrive at the correct response. Interestingly, the small group of reasoners who did learn to correct their errors after receiving feedback showed a stronger conflict detection effect in the pretest block, took more deliberation time after receiving negative feedback, and performed better on the two transfer problems, compared to the group of reasoners who remained biased on all problems. Also, for most participants who improved, correct responding initially occurred during the deliberation (final response) stage. However, on the next problem or on the one after that, they managed to also solve it correctly without deliberation (i.e., already correct at the intuitive response stage) and remained correct throughout the subsequent problems (on both response stages). This suggests that most participants who found the correct answer automatized the reasoning very quickly. 
The last study, presented in Chapter 7, investigated the internal validity of the Actively Open-minded Thinking scale in two samples of Dutch higher education students $(N=930$ and $N=509$ ). This frequently used questionnaire intends to measure the disposition towards rational thinking and has been used to explain individual differences in biased reasoning. Yet, despite its frequent use, it is unclear whether and to what degree the items measure the psychological trait actively open-minded thinking. Mokken scale analyses showed that none of the 41 items in the scale could discriminate sufficiently between respondents with varying latent trait levels. Furthermore, no item-set could be obtained that would allow for validly ordering individuals on the assumed latent trait of actively open-mined thinking, which is a crucial assumption when using it in research. Consequently, it is questionable whether scores on the Actively Open-minded Thinking scale provide insight into the concept it aims to measure.

\subsubsection{DISCUSSION: IMPLICATIONS AND FUTURE DIRECTIONS}

The results presented in Chapter 5, 6, and 7 build on and extend previous research on the (cognitive) mechanisms underlying biased reasoning and provide some suggestions on what might work and why when teaching people to avoid biases in their thinking. Chapter 5 and 6 provide further evidence for the conflict detection effect, showing it occurs also during reasoning on more realistic tasks and when evaluating other people's decisions. This is important because, when teaching to avoid biased reasoning, the goal is to teach how to avoid biased decisions on actual problems and not on the problems that heuristics-and-biases tasks typically address (e.g., judging whether a person is most likely a dentist or rock singer [cf. Frey et al., 2018] is quite far removed from important real-world decisions with far-reaching consequences). The study in Chapter 5 took a first step towards investigating conflict detection in more realistic scenarios and in evaluating other people's decisions. Although, the results need to be replicated and extended with other tasks in other (more ill-structured) reasoning contexts, the finding that people detect conflict also on more realistic (decision-evaluation) tasks offers interesting suggestions for further research into training people to avoid biased reasoning.

Connections between conflict detection and findings on teaching critical thinking. First, Chapter 3 showed that CT-training increased teachers' mental effort ratings in solving transfer tasks, but not their actual reasoning performance. This may indicate - just as for the feedback intervention in Chapter 6 - that the training did affect teachers' error awareness but not their reasoning accuracy on transfer tasks. Note that I make the assumption here that mental effort could be a proxy for conflict detection, which would be interesting to establish in future research. As findings from Chapter 5 and 6 suggest that biased reasoners who show a more pronounced conflict detection effect have a higher chance to start reasoning correctly on a next problem, or to benefit from a minimal intervention such as simple response feedback, conflict detection might be used next to reasoning accuracy as a more sensitive outcome measure. This would be very relevant for future research into the effects of CT-training, as it might (perhaps) indicate a 'readiness' for transfer (i.e., people who show signs of conflict 
detection might benefit more from feedback/instruction than people who do not). Second, given that the conflict detection mechanism also seems to occur during evaluating reasoned decisions of others (Chapter 5), teachers might also detect conflict when evaluating biased reasoning of their students. This may also explain why CT-training had such a large effect on teachers' ability to detect biases in a student product (Chapter 3). If we would assume that teachers already have detected conflict during evaluating students' biased reasoning at a pretest (yet not strong enough to point the biases out explicitly), this would explain why the training helped them to start pointing out even the non-instructed biases explicitly at posttest. It is promising that the skill to detect biases can be trained relatively easily. It would be interesting to investigate in future research whether teachers could also train their students' awareness of reasoning errors.

Future research on conflict detection and dispositions. The current findings (Chapter 5 and 6) suggest that biased reasoners who show a more pronounced conflict detection effect have a higher chance to start reasoning correctly on a next problem, or to benefit from a minimal intervention such as simple response feedback. Hence, this could indicate that a larger detection effect can interpreted as "better" reasoning. This interpretation is, however, highly speculative and probably too simple because a qualitative interpretation of such effect sizes is rather complex. As explained by Frey et al. (2018), a large increase in deliberation time after conflict detection (i.e., large conflict detection effect) does not necessarily indicate good detection or reasoning, but may also reflect inefficient reasoning. Vice versa, a relatively short increase in deliberation time (i.e., small conflict detection effect) does not necessarily indicate inferior detection or reasoning, but can also mean that this person is an efficient reasoner who is less affected by an experienced conflict. Also note that previous research has shown mixed findings on correlations between reasoning accuracy and detection effect size, especially on response time indices (Frey et al., 2018; Pennycook et al., 2015a). To improve CT-training efficacy, it would be interesting to further investigate what the size of an individual's conflict detection signal means, and under what circumstances it would be an adequate predictor of future reasoning accuracy. For example, there are theoretical suggestions that conflict detectors with stronger logical intuitions (e.g., highly automatized numeracy mindware) are more likely to deliberate on their intuitively cued biased response or to even override it and find the logical response (Bago \& De Neys, 2017; De Neys \& Pennycook, 2019). In addition, it may be that those who are more disposed towards rational thinking are more likely to let error detection result into correct reasoning. However, further research into this requires valid measurement instruments of variables like mindware and thinking dispositions which both are quite challenging to assess. As shown in Chapter 7, existing and even frequently used instruments may not measure the intended construct adequately. Consequently, it is important to start developing valid and reliable instruments of such crucial concepts, an issue to which I will return in the next section. 


\subsection{LIMITATIONS}

The research presented in this dissertation has several limitations. I will focus on overarching issues here as study specific limitations have been addressed in the respective chapters. Two more general limitations were due to the fact that studies in the first part were conducted in authentic teaching contexts. First, it was challenging to obtain large teacher samples for the training studies (Chapters 3 and 4), as these required substantial time investment from teachers (which could conflict with their teaching schedules or other professional development activities). Consequently, the conducted experiments could only pick up medium to large sized effects and not more subtle effects. In addition, because it was challenging to recruit large teacher samples, it was not possible to design experiments with multiple conditions that would have allowed for comparing and drawing conclusions on what specific aspects of the training were most effective for which outcomes. Second, because participating in the studies was on voluntary basis and required quite some time and effort investment, self-selection bias may have been present (especially in Chapters 2 and 3), which may have positively affected teachers' average performance level and attitudes. Nevertheless, because most of the current findings were in line with findings from other studies with (larger samples of) student populations, I would not expect that the outcomes would differ when studied in which self-selection bias is not present.

Another limitation of the research reported in this dissertation, and in CT research in general, concerns the measurement of CT. Both in research using standardized CT-tests (Bernard et al., 2008; Bondy et al., 2001; Jacobs, 1999; Leppa, 1997; Loo \& Thorpe, 1999) and in research using heuristic-and-bias tasks (Aczel et al., 2015; Bruine de Bruin et al., 2007; West et al., 2008; the current studies) reliability issues are quite common. For the heuristics-and-biases tests, a potential explanation is that multiple uncorrelated latent factors underlie performance on the test items. Take for example a classical task that is used to measure belief bias in syllogistic reasoning:

Premise 1: All living things need water.

Premise 2: $\quad$ Roses need water.

Conclusion: Roses are living things.

Does the conclusion follow necessarily from the premises?

Performance on such tasks depends not only on the extent to which the conclusion triggers a belief bias, but also on the extent to which a person possesses the requisite mindware. In other words, systematic variance in performance on such tasks can either be explained by a person's belief bias or by his/her logical reasoning skills. If it differs per item why a person got the answer right or wrong and the variables explaining these differences (e.g., belief bias and mindware) do not correlate (strongly), then a problem arises when calculating the reliability for this set of items. The complexity of this problem may increase even more when different task types are included in one test (as done in many previous studies and in 
Chapter 3). Constructing a test with many items that only target one type of bias and require only one type of mindware (Chapter 4) can solve the low reliability issue, but still does not tell whether the test assessed one latent construct or multiple constructs. Moreover, a test including only items on belief bias is a rather restricted operationalization of the theoretical idea behind the ability to avoid bias in reasoning. For example, in their book "The Rationality Quotient", Stanovich, West, and Toplak (2016) distinguish between 20 subtests that should together reflect people's rational thinking (defined in terms of avoiding cognitive biases). Although this book made an important contribution by classifying all different types of rational thinking into one theoretical model, there is no psychometric evidence (yet) for the internal validity of this model. Taken together, an important question for future research to address is whether the ability to avoid bias in reasoning and decision-making (or rational thinking) can be considered as a single construct, as multiple correlated constructs without one higher-order factor, or whether there are different unrelated constructs. More advanced psychometric approaches (e.g., item response theory as applied in Chapter 7) can provide an answer to this.

These measurement issues also raise the question of what the tests in this dissertation research and in previous research by others have actually measured. I would argue that despite the issues raised - the findings do say something about the reasoning biases that were assessed, since the used heuristics-and-biases tasks represent models of how people reason in all kinds of contexts. That is, the thinking biases leading to incorrect reasoning and decisions on these tasks, are presumably the same thinking biases that lead to incorrect reasoning and decisions in the real world. Taking the belief bias as example again: people are not only systemically biased by their beliefs when dealing with syllogisms, they are also systemically biased by their beliefs when reasoning about political information in their actual lives (Thibodeau et al., 2015). As such, a correct, non-biased response on a heuristics-and-bias task is always favorable over an incorrect response (and also related to better everyday reasoning; Pennycook et al., 2015b; Toplak et al., 2017). Therefore, finding improved performance on these tasks due to training or feedback is meaningful, especially when the tasks are embedded in more realistic contexts (e.g., detecting and explaining biases in excerpts from student papers). In addition, in conducting this research I have attempted to approach the construct in various ways by using different task formats (classical heuristics-and-biases tasks, tasks to asses bias in students' written arguments, and tasks involving more realistic decision-making or decision-evaluation situations), with different response formats (multiple-choice, free-response, and the two-response paradigm), while taking multiple response measures into account (performance, confidence, response time, mental effort, and conflict detection), and tried to validate an existing measure of the disposition towards actively open-minded thinking (without success). The combination of these measures provided a rich insight into how people reason. 


\subsection{CONCLUSION AND RECOMMENDATIONS FOR PRACTICE}

The studies in this dissertation provide first evidence for the trainability of teachers' own ability to avoid bias, their ability to detect bias in students' reasoning, and their ability to clearly explain to students why their reasoning is biased. These results imply that it is important for educational practice to start focusing explicitly on improving teachers' ability to provide feedback on students' biased reasoning, which requires improving their ability to avoid, detect, and explain biased reasoning. Feedback is a powerful way to improve learning and concerns an instructional intervention that can be integrated throughout the whole curriculum. The research presented in this dissertation shows that the essential cognitive skills that teachers need to provide feedback on students' reasoning biases can and need to be trained. However, it also showed that transfer to explaining non-instructed biases does not occur easily. Moreover, the essential attitudes necessary for bringing such skills into the teaching practice may not improve along with improved cognitive skills. Thus, teachers training on CT, and future research into the effectiveness of such a training, should not only focus on improving relevant CT-teaching-skills, but should also explicitly focus on fostering teachers' attitudes towards teaching these skills. One way to achieve this could be to let teachers decide what specific CT-skills have priority in their educational program, based on what is important for the professional domain in which the students end up working, and on what reasoning biases they want to learn to provide feedback. This should be a carefully designed decision-making process, resulting in concrete learning goals of which all teachers recognize the relevance. Subsequently, scheduling some training sessions combined with enough time for retrieval practice may be sufficient for teachers to master the targeted feedback skills and feel competent in providing feedback in class, thereby making an impact on the teaching of CT in higher education. 
Nederlandse samenvatting

(Summary in Dutch)

Kritisch denken doceren

in het hoger onderwijs:

bias in redeneren leren vermijden,

herkennen en vitleggen 
Het kritisch denken van studenten bevorderen is een belangrijke doelstelling van het hoger onderwijs. Docenten spelen een cruciale rol in het realiseren van deze doelstelling. Desondanks is er weinig bekend over hoe docenten het beste ondersteund kunnen worden in het succesvol vervullen van deze rol. Het hoofddoel van dit proefschrift was daarom een eerste stap te zetten in het onderzoek naar de vraag hoe we docenten uit kunnen rusten met de kennis en vaardigheden die nodig zijn voor het onderwijzen van een essentieel aspect van kritisch denken: het vermogen om bias in redeneren en besluitvorming te vermijden.

\section{HET VERMIJDEN VAN BIAS}

Een cognitieve bias of "cognitieve vertekening" is een systematische fout in het menselijk denken. Door de zogeheten overtuigingsbias (belief bias) bijvoorbeeld, zijn we snel geneigd een onlogische conclusie te accepteren omdat we al overtuigd zijn van de waarheid van die onlogische conclusie. Deze neiging wordt duidelijk in het onderstaande redeneervraagstuk. Het betreft een logische redenering, bestaande uit twee stellingen en een conclusie. De vraag is of de conclusie logisch volgt uit de gegeven stellingen:

Alle bloemen hebben water nodig.

Rozen hebben water nodig.

Rozen zijn dus bloemen.

Het antwoord is nee. We weten allemaal dat rozen bloemen zijn, maar de vraag was of de conclusie hierboven logisch volgt uit de stellingen en dat is niet het geval. De eerste stelling impliceert niet noodzakelijkerwijs dat alles wat water nodig heeft enkel een bloem kan zijn. Daarom mag uit de tweede stellingname dat rozen water nodig hebben niet logisch geconcludeerd worden dat rozen bloemen zijn. Hoewel dit vraagstuk niet op ingewikkelde logica berust, antwoordt toch maar liefst $70 \%$ van de universitair studenten in verschillende onderzoeken ten onrechte dat de conclusie logisch uit de stellingen volgt (Markovits \& Nantel, 1989; Sá et al., 1999). De sterke overtuiging of, in dit geval, de kennis dat rozen bloemen zijn, belemmert hier het vermogen om logisch te redeneren. Wanneer we in bovenstaande taak het woord "rozen" door "tijgers" vervangen, treedt de logische denkfout niet meer op. Niet alleen op taken als hierboven, maar ook in het dagelijks leven leidt deze overtuigingsbias tot fouten in ons redeneren. Mensen laten zich bijvoorbeeld systematisch misleiden door hun eigen overtuigingen wanneer ze redeneren over politieke informatie die ze lezen (Thibodeau et al., 2015). Naast logisch redeneren zijn we ook vrij onbedreven in het inschatten van kansen. We laten ons hierbij al gauw misleiden door de informatie over één 
uniek geval en nemen de benodigde basisinformatie voor het schatten van de relatieve kans op dit unieke geval te weinig in overweging. Deze bias berust dus op de neiging om de weging van basiskansen te verwaarlozen (base-rate neglect bias). Deze neiging komt bij velen tot uiting in onderstaand redeneervraagstuk:

In een onderzoek werden 1000 mensen getest. Onder de deelnemers waren 5 vrouwen en 995 mannen. Sasha is een willekeurig gekozen deelnemer aan het onderzoek. Sasha gaat graag naar moderne dansles op vrijdagavond. Na afloop drinkt Sasha vaak nog een cocktail, het liefste een Aperol Spritz. Sasha wordt door iedereen erg lief gevonden.

Wat is het meest waarschijnlijk?

Sasha is een vrouw

Sasha is een man

Het meest waarschijnlijk is dat Sasha een man is. Hoewel de persoonsbeschrijving eerder past bij het stereotype beeld van een vrouw, zijn er genoeg mannen die graag naar moderne dansles gaan en cocktails drinken. En belangrijker: omdat de steekproef voor $99.5 \%$ uit mannen bestaat, is het veel waarschijnlijker dat Sasha man is dan vrouw. Het oplossen van dit vraagstuk vereist geen complexe berekening, maar een dergelijke vraag wordt toch door zo'n $80 \%$ van de universitair studenten onjuist beantwoord (De Neys et al., 2011). De inschatting wordt hier te sterk gebaseerd op de associatie met vrouwen, die de beschrijving van Sasha oproept. Daarnaast wordt de basisinformatie over de groepssamenstelling, benodigd voor het schatten van de relatieve kans dat Sasha vrouw is, te weinig in overweging genomen. Deze basis-kans verwaarlozingsbias komt ook vaak terug in ons dagelijks redeneren. De meeste mensen maken zich bijvoorbeeld meer zorgen over de kans op terrorisme dan over de gevaren die statistisch veel waarschijnlijker zijn, zoals bijvoorbeeld het krijgen van een auto-ongeluk (Sunstein, 2003).

Hoewel bias in het redeneren inherent is aan de menselijke cognitie en in veel gevallen vrij onschuldig is, kan het ook leiden tot onjuiste beslissingen die soms ernstige gevolgen hebben. Bijvoorbeeld wanneer een rechter de aangeklaagde schuldig acht op basis van een verkeerde weging van het statistische bewijs hiervoor (Thompson \& Schumann, 1987), wanneer artsen een diagnostische fout maken doordat ze eerder die dag iets hebben gelezen over een specifiek, onwaarschijnlijk ziektebeeld in de media (Schmidt et al., 2014) of wanneer ouders besluiten hun kinderen niet te vaccineren omdat ze zich zeldzame gevallen herinneren waarin vaccins hebben gefaald of tot complicaties leidden (Smith, 2017). Dit zijn slechts een paar voorbeelden, maar de lijst van cognitieve biases waar mensen aan ten prooi kunnen vallen lijkt oneindig lang (Kahneman, 2011). Het is dan ook van belang om te onderzoeken of, en hoe, we bias in het redeneren en beslissen, zoveel mogelijk, kunnen tegengaan; met name in het hoger onderwijs waarin toekomstige professionals worden opgeleid. Willen we studenten leren om bias in hun redeneren te vermijden, dan zullen docenten hun deze kritische denkvaardigheid expliciet moeten onderwijzen. 


\section{KRITISCH DENKEN IN DIT PROEFSCHRIFT}

Om kritisch denken te kunnen onderwijzen, zijn de volgende drie variabelen, die centraal staan in dit proefschrift, belangrijk: (1) kritische denkvaardigheden van docenten zelf, in dit proefschrift geoperationaliseerd als het vermogen van docenten om bias in hun redeneren te vermijden; (2) vaardigheden om kritisch denken te onderwijzen, waaronder het vermogen van docenten om bias in redeneringen van studenten te herkennen en te kunnen uitleggen waarom de conclusie in deze redeneringen niet juist is; en (3) een positieve houding van docenten ten aanzien van het onderwijzen van kritisch denken, waaronder het erkennen van de relevantie om kritisch denken te onderwijzen en het gevoel hebben hier competent in te zijn. Het onderzoek in het eerste deel van dit proefschrift (hoofdstukken 2, 3 en 4) had betrekking op de hoofdvraag "Hoe kunnen docenten in het hoger onderwijs uitgerust worden met de kennis en vaardigheden die nodig zijn voor het onderwijzen van kritisch denken?". Het tweede deel (hoofdstukken 5, 6 en 7) bevatte drie aanvullende studies over cognitieve processen (hoofdstukken 5 en 6) en disposities (hoofdstuk 7) die ten grondslag liggen aan bias in het redeneren. In deze samenvatting presenteer ik de hoofdbevindingen van de zes studies en sluit ik af met het antwoord dat ze gezamenlijk op de hoofdvraag geven.

\section{DEEL 1: KRITISCH DENKEN ONDERWIJZEN}

\section{ONDERZOEKSRESULTATEN}

Het doel van de studie in hoofdstuk 2 was om te onderzoeken welke (hbo-)docentkenmerken samenhangen met betere kritische denkvaardigheden en met een meer positieve houding ten aanzien van het onderwijzen van kritisch denken. Kritische denkvaardigheden werden gemeten met een verlengde versie van de zogeheten Cognitieve Reflectie Test (CRT; Toplak et al. 2014a), waarmee docenten $(N=263)$ werden getest op hun vermogen om bias in hun redeneren te vermijden op zeven korte rekenvraagstukken. De houding ten aanzien van het onderwijzen van kritisch denken werd gemeten met een vragenlijst over hoe relevant docenten het onderwijzen van kritisch denken vonden en hoe competent ze zichzelf hierin voelden. De resultaten van een structureel vergelijkingsmodel toonden aan dat docenten die (1) sterker de neiging (dispositie) hadden om kritisch na te willen denken, (2) die lesgaven binnen een technisch domein (vergeleken met maatschappelijk en economisch domein) en (3) die een hoger opleidingsniveau hadden, gemiddeld genomen beter presteerden op de CRT. Daarnaast beoordeelden docenten die geneigd waren om meer moeite te doen in kritisch denken het onderwijzen van kritisch denken als relevanter dan docenten die minder sterk die neiging hadden. De CRT-prestatie was echter niet gerelateerd aan hoe relevant docenten het onderwijzen van kritisch denken vonden. Tenslotte was het niet zozeer hun daadwerkelijke CRT-prestatie, maar het vertrouwen dat docenten hadden in hun prestatie, dat voorspelde hoe competent ze zichzelf voelden in het onderwijzen van kritisch denken, waarbij een groter vertrouwen samen ging met een hogere mate van ervaren competentie. 
De quasi-experimentele studie in hoofdstuk 3 onderzocht de effecten van een training voor hbo-docenten gericht op (het onderwijzen van) kritisch denken. De training bestond uit drie sessies van elk drie uur, verspreid over zes weken. De eerste sessie was gericht op het trainen van de kritische denkvaardigheden van docenten zelf. Docenten ontvingen aan de hand van verschillende redeneervraagstukken expliciete instructies over een aantal veelvoorkomende biases en hoe deze te vermijden, gevolgd door oefenvraagstukken die ze zelf moesten lossen. De tweede en de derde sessie waren gericht op het versterken van de vaardigheden in het lesgeven in kritisch denken en de houding ten aanzien daarvan. De docenten oefenden met extra redeneervraagstukken en ontwierpen, gebaseerd op de oefenvraagstukken, zelf een domein-specifiek vraagstuk. Daarnaast werd de relevantie van het onderwijzen van kritisch denken bediscussieerd en werden er manieren besproken waarop kritisch denken in het lesgeven geïntegreerd kan worden. De 32 docenten die deelnamen aan de training, de experimentele conditie, maakten voorafgaand en direct na afloop van de eerste en laatste trainingssessie een test waarmee hun eigen kritische denkvaardigheden, twee benodigde vaardigheden voor het onderwijzen ervan en hun houding ten aanzien van het onderwijzen van kritisch denken werd gemeten. Op vergelijkbare tijdstippen werd dezelfde test gemaakt door een groep van 22 docenten die geen van allen de training volgden, de controleconditie. De resultaten wezen uit dat de training een groot en positief effect had op het vermogen van docenten om bias in hun redeneren te vermijden op de leertaken, dat wil zeggen, op het type vraagstukken dat in de training aan bod was gekomen. De training had echter geen effect op hun prestatie op transfertaken, dat wil zeggen, op hun vermogen om gerelateerde, maar niet-getrainde biases in het redeneren te vermijden. Met betrekking tot vaardigheden om kritisch denken te onderwijzen lieten de getrainde docenten een sterke verbetering zien in het herkennen van biases die voorkwamen in een kort studentverslag, specifieker de managementsamenvatting van een afstudeeronderzoek. Hoewel de getrainde docenten, in tegenstelling tot de niet-getrainde docenten, ook iets beter scoorden in het uitleggen waarom de conclusie in die redeneringen onjuist was, was hun prestatie nog steeds vrij laag: bij meer dan de helft van de gedetecteerde biases gaven ze geen goede uitleg, gemiddeld genomen. Tenslotte had de training geen positief effect op de houding ten aanzien van het onderwijzen van kritisch denken. Al voordat de training had plaatsgevonden, gaven bijna alle docenten in beide condities aan het onderwijzen van kritisch denken relevant te vinden, dat wil zeggen, ze waren het gemiddeld genomen eens met alle stellingen die deze relevantie adresseerden. Hun waargenomen relevantie nam niet verder toe of af na het volgen van de training. Docenten achtten zichzelf doorgaans ook al redelijk competent in het onderwijzen van kritisch denken, dat wil zeggen, ze waren het gemiddeld genomen "een beetje eens" met stellingen die hun persoonlijke competentiegevoel adresseerden. Na afloop van de eerste trainingssessie daalde de ervaren competentie in de experimentele conditie, maar deze steeg na afloop van de laatste trainingssessie weer tot hetzelfde niveau als in de (stabiel gebleven) controle conditie.

De training in hoofdstuk 3 bleek slechts een klein effect te hebben op de vaardigheid van docenten om studenten uitleg te geven betreft de bias in hun redeneren. Dit is echter een cruciaal aspect van het lesgeven in kritisch denken: als een redenering niet klopt dan moeten docenten feedback kunnen geven aan hun studenten. Daarom werd in hoofdstuk 4 middels 
een experiment onderzocht of het vermogen van docenten om bias in studentredeneringen te kunnen uitleggen, verbeterd kon worden door hen hier expliciet op te trainen. Docenten in de controleconditie $(n=19)$ namen deel aan een trainingssessie die enkel gericht was op het vermijden van bias in hun eigen redeneren (vergelijkbaar met de eerste trainingssessie in hoofdstuk 3). Docenten in de experimentele conditie $(n=18)$ volgden deze training ook, maar kregen daarnaast een aanvullende online training waarin ze instructie ontvingen over en oefenden met het geven van feedback op biases die voorkwamen in studentverslagen, specifieker alinea's uit beroepsproducten. Voorafgaand aan, direct na afloop van, en twee weken na de training maakten docenten in beide condities een test waarin ze aan fictieve studenten uitleg moesten geven over de bias in hun redeneren in geschreven verslagen. Tevens werden de docenten bevraagd op hun houding ten aanzien van het onderwijzen van kritisch denken. Docenten in de experimentele conditie presteerden direct na afloop van de uitlegtraining beter op de leertaken dan voorafgaand aan de training, dat wil zeggen, op het kunnen uitleggen van de biases waarmee ze hadden geoefend. Ze presteerden tevens beter dan de docenten in de controleconditie die, na afloop van de training over bias in het eigen redeneren, zelfs helemaal geen vooruitgang lieten zien in het uitleggen van de biases. Twee weken later bleek dat docenten in de experimentele conditie hun verbetering in het kunnen uitleggen van bias (t.o.v. voorafgaand aan de training) hadden weten te behouden. Verrassend was echter dat ook de docenten in de controleconditie, ogenschijnlijk spontaan, vooruitgegaan waren. In beide condities vond slechts een minimale verbetering plaats in het uitleggen van biases die niet in de uitlegtraining aan bod was gekomen, maar wel op vergelijkbare onderliggende principes berustten. Met andere woorden, er was nauwelijks transfer van getrainde naar niet-getrainde taken. Tenslotte vonden docenten het onderwijzen van kritisch denken voorafgaand aan de training al relevant en voelden ze zich hier ook al redelijk competent in. Deze houding veranderde verder niet over de drie testmomenten heen.

\section{CONCLUSIE}

De studies in de hoofdstukken 2, 3 en 4 begaven zich op een tot dusver nauwelijks verkend terrein: het kritisch denken van docenten en hun vaardigheden dit te onderwijzen, in het bijzonder, de kritische denkvaardigheid om bias in het redeneren te vermijden. De resultaten van deze studies tonen aan dat training zowel de kritische denkvaardigheden van docenten als hun vaardigheden om dit te onderwijzen kan verbeteren. Immers, de twee onderzochte trainingen hadden een groot leereffect op de getrainde (onderwijs)vaardigheden en hoofdstuk 3 wees tevens uit dat training gericht op het vermijden van bias in het eigen redeneren een groot effect had op de onderwijsvaardigheid bias in het redeneren van studenten te herkennen. Er vond dus transfer plaats van het redeneren in de getrainde context (bias op redeneervraagstukken vermijden) naar het toepassen van de geleerde principes in een nieuwe, ongetrainde context (bias in studentverslagen herkennen). Hoofdstuk 3 en 4 laten echter ook zien docenten niet of nauwelijks kunnen uitleggen waarom er een bias in het redeneren van studenten zit wanneer ze hier niet expliciet op getraind zijn. Voor het lesgeven is juist deze vaardigheid, om feedback op bias in het redeneren van studenten te kunnen geven, van 
cruciaal belang. We kunnen niet van studenten verlangen dat ze leren bias te vermijden zolang docenten ze niet uit kunnen leggen wanneer en waarom er een bias in hun redeneren zit en hoe ze hun redenering kunnen verbeteren. De resultaten geven dus aan dat het noodzakelijk om docenten te trainen op dergelijke vaardigheden. Hoewel het vermijden, herkennen en uitleggen van bias te trainen is, tonen de onderzoeksresultaten ook aan dat transfer van een getrainde bias naar nieuwe (enigszins gerelateerde) biases niet zo maar optreedt. Dergelijke transfer zou wel zeer wenselijk, zo niet noodzakelijk, zijn, omdat het praktisch onhaalbaar is om docenten (en studenten) te trainen in het vermijden van alle bestaande biases. Tenslotte geven alle drie de studies aan dat de houding van docenten ten aanzien van het onderwijzen van kritisch denken al behoorlijk positief was, maar niet automatisch positiever wordt wanneer docenten beter worden in de benodigde (onderwijs)vaardigheden. Hoewel een positieve houding voorafgaand aan training wenselijk is, is ook aandacht voor het bekrachtigen van deze houding van groot belang. Een hoge, maar onveranderde vragenlijstscore op deze houding kan ons immers niet vertellen of docenten het onderwijzen van de getrainde vaardigheden al "relevant genoeg" vinden en of ze zichzelf hier al "voldoende competent" in voelen. Deze twee houdingsaspecten bepalen echter wel in grote mate of docenten de getrainde vaardigheden op het gebied van (lesgeven in) kritisch denken daadwerkelijk in hun onderwijspraktijk zullen toepassen.

\section{DEEL 2: COGNITIEVE PROCESSEN EN DISPOSITIES TEN GRONDSLAG AAN BIAS}

\section{ONDERZOEKSRESULTATEN}

De studies in het tweede deel van dit proefschrift waren gericht op cognitieve processen en disposities die ten grondslag liggen aan bias in het redeneren. Hoofdstuk $\mathbf{5}$ bouwde voort op recent onderzoek wat aantoont dat de meeste mensen die aan een bias ten prooi vallen zich hier toch enigszins bewust van lijken te zijn; ze vertonen tekenen van conflictdetectie (De Neys \& Glumicic, 2008; Frey et al., 2018; Pennycook et al., 2015a). Conflictdetectie wordt doorgaans gemeten aan de hand van reactietijden (hoe lang deelnemers erover doen om een vraagstuk op te lossen) en aan de hand van het vertrouwen dat deelnemers rapporteren te hebben in de accuratesse van hun gegeven antwoord. Deze twee variabelen worden in verband gebracht met de prestatie van deelnemers op zogeheten conflicttaken en geen-conflicttaken. Conflicttaken zijn zo ontworpen dat ze een bepaald intuïtief antwoord, een heuristiek, oproepen dat in conflict is met de logische- of statistische taakinformatie die in acht genomen moet worden, zoals het geval is bij de voorbeeldtaken hierboven. Daarentegen zijn geen-conflicttaken ontworpen om precies dezelfde heuristiek op te roepen maar, in dit geval, vormt de opgeroepen heuristiek geen conflict met de logische of statistische informatie in de taak. Een geen-conflictversie van het Sasha-vraagstuk hierboven zou refereren naar een steekproef van 995 vrouwen en 5 mannen, zodat het beeld dat de persoonsbeschrijving van Sasha oproept in lijn is en geen conflict vormt met de statistische taakinformatie. Als mensen bij het oplossen van het Sasha-vraagstuk enkel op de persoonsbeschrijving afgaan, zonder de statische informatie in overweging te nemen, dan 
zouden ze even zeker moeten zijn over hun incorrecte antwoord op de conflictversie van het vraagstuk als over hun correcte antwoord op de geen-conflictversie. Je zou daarnaast dan ook verwachten dat hun reactietijd op beide taakversies even lang is. Onderzoek heeft inmiddels echter veelvuldig aangetoond dat de meeste mensen langer doen over hun conflicttaak en minder vertrouwen hebben in hun accuratesse bij het geven van een incorrect antwoord op conflicttaken dan bij het geven van een correct antwoord op geen-conflicttaken. Met andere woorden, de meeste mensen lijken aan te voelen dat hun heuristieke antwoord op de conflicttaak mogelijk niet correct is: ze vertonen tekenen van conflictdetectie.

Een belangrijke tekortkoming in het onderzoek naar conflictdetectie is dat dit cognitieve proces enkel is onderzocht aan de hand van klassieke - niet erg realistische redeneervraagstukken. Omdat beslissen of Sasha hoogstwaarschijnlijk man of vrouw is nog vrij ver af staat van belangrijke beslissingen die doorgaans in het dagelijks leven worden genomen, was nog niet bekend of conflictdetectie ook plaatsvindt in meer realistische beslissituaties. De studie in hoofdstuk $5(N=159)$ onderzocht daarom het conflictdetectie-effect tijdens het redeneren op langere, complexere en meer realistische vraagstukken en vergeleek hierbij twee vormen van redeneren: besluitvorming (bias in eigen beslissing vermijden, zoals bij de vraagstukken hierboven) en besluitevaluatie (bias in de beslissing van iemand anders herkennen, een taak waar docenten dagelijks mee te maken hebben wanneer ze het werk van hun studenten beoordelen). De onderzoeksresultaten toonden aan dat het conflictdetectie-effect ook plaatsvond wanneer mensen redeneerden op de realistische vraagstukken (zie hoofdstuk 5 voor voorbeelden). Deelnemers vertoonden conflictdetectie tijdens het redeneren op zowel besluitvormings- als op besluitevaluatietaken. Daarnaast was het zo dat deelnemers met een groter conflictdetectie-effect (een groter verschil in het gerapporteerde vertrouwen of in de reactietijd tussen conflict- en geen-conflicttaken) gemiddeld genomen een hogere totaalscore hadden op de redeneertaken dan deelnemers die een kleiner conflictdetectie-effect lieten zien. Dit onderzoek leverde het eerste, voorzichtige, bewijs op dat het conflictdetectieproces ook van toepassing kan zijn op besluitvorming en besluitevaluatie in meer realistische situaties. Deze resultaten zijn in het bijzonder relevant voor (onderzoek naar) redeneren in contexten waarin het evalueren van andermans redeneren belangrijk is. Bijvoorbeeld in het onderwijs waar docenten de argumenten van hun studenten moeten beoordelen of in de geneeskunde waar artsen de initiële diagnoses van anderen zorgvuldig moeten checken.

De studie in hoofdstuk 6 onderzocht het redeneren op het populaire knuppel-balraadsel (the bat-and-ball problem; een van de taken uit de CRT in hoofdstuk 2). Het doel was te onderzoeken wat de impact was van minimale prestatiefeedback op het vermijden van bias in het redeneren op (vergelijkbare versies van) het knuppel-balraadsel. De prestatiefeedback hield in dat deelnemers na het oplossen van een vraagstuk zagen of hun gegeven antwoord goed of fout was, zoals ook in het onderwijs veelvuldig gebeurt. Resultaten van twee experimenten $(N=112$ en $N=80)$ wezen uit dat de feedback het redeneren op het knuppel-balraadsel niet significant verbeterde. Uit exploratieve analysen bleek dat deelnemers wel een groter conflictdetectie-effect vertoonden na ontvangst van feedback op hun incorrecte antwoorden, gemeten aan de hand van hun reactietijden. Dit wijst erop dat de 
meeste deelnemers de feedback wel verwerkten, maar dat het ze niet hielp tot het correcte antwoord te komen. Er was een kleine groep deelnemers die na feedback wel verbeterde. Interessant was dat deze groep in vergelijking met de groep die niet verbeterde, al een groter conflictdetectie-effect vertoonde op een pretest waarin ze nog geen feedback ontvingen. Daarnaast deed deze groep langer over het geven van een antwoord na het ontvangen van de feedback en presteerde beter op twee transfertaken.

De laatste studie, gepresenteerd in hoofdstuk 7, onderzocht de interne validiteit van de Actief-Open-Minded-Denken-schaal. Deze vragenlijst beoogt de dispositie tot rationeel denken te meten en is veelvuldig gebruikt om individuele verschillen in bias in het redeneren te verklaren. Ondanks het frequente gebruik was tot dusver nog onduidelijk of en in welke mate de vragen van deze schaal daadwerkelijk de psychologische eigenschap "actief open-minded zijn" meten. De Nederlandse vertaling van deze vragenlijst was ingevuld door Nederlandse hbo-studenten $(N=930$ en $N=509)$. Mokken-schaalanalyses wezen uit dat geen van de 41 vragen in de schaal voldoende onderscheidend vermogen had om verschillen tussen respondenten aan te kunnen tonen op de latente trek die de schaal beoogt te meten, namelijk de eigenschap actief open-minded te zijn. Er was ook geen subset aan vragen die het wel mogelijk maakte respondenten valide te ordenen op de veronderstelde latente de trek. Dit is echter een cruciale voorwaarde om de schaal te kunnen gebruiken voor onderzoeksdoeleinden en de resultaten betekenisvol te kunnen interpreteren. Het is daarom zeer de vraag of scores op de Actief-Open-Minded- Denken-schaal inzicht geven in het concept dat de vragenlijst beoogt te meten.

\section{CONCLUSIE}

Het onderzoek in de hoofdstukken 5, 6 en 7 bouwde voort op eerder onderzoek naar (cognitieve) processen die ten grondslag liggen aan bias in het redeneren. De resultaten vergroten niet alleen ons inzicht in hoe mensen redeneren, maar geven ook enkele suggesties over wat mogelijk werkt en waarom bij het leren van de vaardigheid om bias in het redeneren te vermijden. Hoofdstuk 5 en 6 geven evidentie voor het conflictdetectie-effect en hoofdstuk 5 laat zien dat dit effect ook optreedt tijdens redeneren op realistische vraagstukken, zowel tijdens besluitvorming als tijdens besluitevaluatie. Dit laatste is hoopvol voor de onderwijspraktijk, omdat dit zou kunnen betekenen dat docenten ook conflict detecteren wanneer ze beslissingen van hun studenten evalueren. Als het mogelijk is docenten te trainen om hun eigen conflictdetectieproces te herkennen, bijvoorbeeld tijdens het beoordelen van studentverslagen, dan zou dit een interessante trainingsbenadering kunnen zijn voor het verbeteren van hun vermogen bias expliciet te detecteren. Beide hoofdstukken wijzen er daarnaast op dat de grootte van het conflictdetectie-effect een positieve voorspeller is van de kans om bias op volgende redeneervraagstukken wel te vermijden. Dit maakt het een interessante uitkomstmaat om naast taakprestatie mee te nemen in onderwijsonderzoek naar de effecten van training gericht op het vermijden van bias. Een belangrijke kanttekening hierbij is wel dat de precieze betekenis van de effectgrootte van conflictdetectie vooralsnog onduidelijk is en tevens vrij complex. Toekomstig onderzoek zou zich dan moeten richten op 
het vergroten van het inzicht in deze betekenis, bijvoorbeeld door de effectgrootte in verband te brengen met relevante variabelen zoals bijvoorbeeld denkdisposities. Dit vraagt echter om betrouwbare en valide meetinstrumenten en, zoals aangetoond in hoofdstuk 7, dat is nog best een uitdaging.

\section{AANBEVELING VOOR DE PRAKTIJK}

Het hoofddoel van dit proefschrift was een eerste stap te zetten in het onderzoek naar de vraag hoe we docenten uit kunnen rusten met de kennis en vaardigheden die nodig zijn voor het onderwijzen van een belangrijk aspect van kritisch denken: het vermogen om bias in redeneren en besluitvorming te vermijden. Gezamenlijk bieden de studies uit dit proefschrift een aanzet tot een antwoord op die vraag en geven ze richting aan verder (praktijkgericht) onderzoek naar deze vraag.

Om studenten te leren bias te vermijden is het belangrijk dat de onderwijspraktijk zich expliciet gaat richten op het verbeteren van de cruciale vaardigheid van docenten om feedback te kunnen geven op bias in het redeneren van hun studenten. Feedback is een krachtige manier om het leren van studenten te bevorderen en betreft een onderwijsinterventie die relatief eenvoudig in het hele curriculum geïntegreerd kan worden. Het geven van goede feedback doet een beroep op het vermogen van docenten om bias in hun eigen te redeneren te vermijden, bias te herkennen in het redeneren van hun studenten en vervolgens aan hun uit te leggen hoe ze hun redenering kunnen verbeteren. Het onderzoek in dit proefschrift wijst uit dat het niet alleen mogelijk maar ook nodig is om docenten in deze vaardigheden te trainen. Praktische implementatie van zo'n training vereist wel dat de houding die docenten hebben ten aanzien van het onderwijzen van de beoogde vaardigheden serieus wordt genomen: docenten moeten deze relevant vinden voor hun onderwijspraktijk en zich na afloop van training ook voldoende competent voelen om ermee aan de slag te gaan. Een mogelijke manier om dit te bereiken, die in toekomstig onderzoek nader onder de loep genomen zou kunnen worden, is door docenten zelf te laten beslissen welke kritische denkvaardigheden prioriteit hebben binnen hun opleiding en op welke biases ze willen leren feedback te geven, uitgaande van de beroepscontext waarvoor wordt opgeleid. Dit moet een zorgvuldig ontworpen besluitvormingsproces zijn, resulterend in concrete leerdoelen waarvan alle docenten de relevantie onderkennen. Het plannen van enkele trainingssessies, in combinatie met het vrij roosteren van voldoende tijd voor docenten om de getrainde vaardigheden onder de knie te krijgen en zich hierin competent te voelen, zou al een impact kunnen hebben op het doceren van kritisch denken in het hoger onderwijs. 
Supplementary Materials 


\section{SUPPLEMENTARY MATERIALS - CHAPTER 3}

\subsection{EXAMPLE ITEMS HEURISTICS-AND-BIASES TASKS}

Below, we translated an example item of each task category administered at the pretest, intermediate test, and posttest. Examples of the other items can be found in the references in our method section or through contacting the first author.

\section{Learning Tasks.}

\section{Belief bias in logic reasoning (cf. Evans, 2002.).}

Premise 1: No lawyers are straightforward

Premise 2: $\quad$ Some crooks are straightforward

Conclusion: Some lawyers are no crooks

Given that both premises are true,

a. the conclusion follows logically from the premises

b. the conclusion does not follow logically from the premises

\section{Correct answer: $\mathrm{b}$}

The important thing to notice here is that one does not confuse the believability of the conclusion with the logical validity of the conclusion. For more information see Evans (2002, p. 983).

\section{Base-rate neglect in probability estimation (cf. Stanovich et al. 2016).}

Imagine you started a new web shop and you need to gain brand awareness among your target group. You are considering paid advertising through social media. What information would you want to have in order to estimate the probability that your web shop will gain brand awareness among your target group given that you use paid advertising through social media? Below are four pieces of information that may or may not be relevant for determining the probability. Please indicate all of the pieces of information and only those pieces of information that are necessary to determine the probability.

a.\% web shops that used paid advertisement through social media, of all web shops that gained brand awareness among their target group

b. $\%$ of web shops that gained brand awareness among their target group

c. $\%$ of web shops that did not gain brand awareness among their target group

d. \% web shops that used paid advertisement through social media, of all web shops that did not gain brand awareness among their target group

Correct answer: $\mathrm{a}+\mathrm{b}+\mathrm{d}$ or $\mathrm{a}+\mathrm{c}+\mathrm{d}$ (we counted $\mathrm{a}+\mathrm{b}+\mathrm{c}+\mathrm{d}$ also as correct). The important thing to notice here is that one should not overrate the first piece of information (a) as this 
percentage is uninformative for the probability estimation as long as you do not know how many unsuccessful web shops used paid advertisement as well (d). For more information, see Stanovich \& West (Stanovich \& West, 2000, p. 654) and Stanovich, West, and Toplak (2016, p. 337)

\section{Transfer Tasks.}

\section{Confirmation bias in logic reasoning (cf. Evans, 2002).}

Each of the four cards below have an image on one side and a number on the other side. The following rule applies to the cards:

'If there is a clover on one side, then there is a 9 on the other side'.

Choose those cards and only those cards that need to be turned over in order to decide whether the rule is true or false.

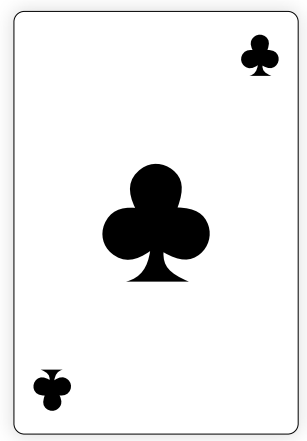

a.

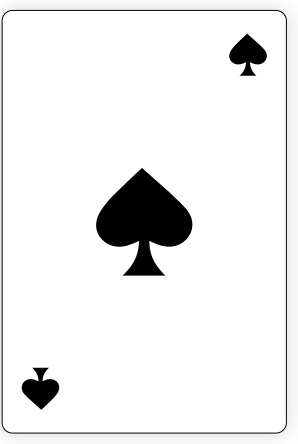

b.

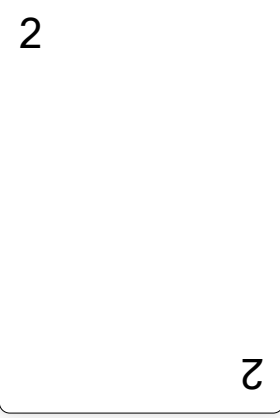

c.
9

\section{6}

Correct answer: $\mathrm{b}$ and $\mathrm{c}$.

The important thing to notice here is that one should not only confirm the logical rule (card b must have a 9 on the other side) but also look for falsification of the rule (card c cannot have a clover on the other side). For more information, see Evans (2002, p. 984) and Gigerenzer and Hug (1992).

\section{Covariation detection problem in probability estimation (cf. Heijltjes et al., 2014).}

You are an entrepreneur and your company is on the brink of bankruptcy. Your neighbor tells you about Corporate Fixer: a company that specializes in solving business problems. 'They do fan-tas-tic work', he says, 'the company of a good friend of mine became extremely successful after their help!' You visit their website and find out that the services of Corporate Fixer are quite pricey. You are prepared to pay the price, provided that you have a better chance of solving your business problems with their help than without any help. On an independent comparison website, you see that (a) 188 companies received help from corporate fixer and solved their business problems, (b) 95 companies did not receive help and solved their problems, (c) 90 companies received help without solving 
their business problems, and (d) 25 companies did not receive help and did not solve their problems:

Business problems solved Business problem unsolved Help from Corporate fixer No help from Corporate fixer 188 95

90 25

Based on this information, would you commission from Corporate fixer or not?

a. yes

b. no

\section{Correct answer: $b$}

The important thing to notice here is that one should to evaluate the information given in a $2 \times 2$ contingency table equally and surpress the tendency to focus on the large number in cell A. For more information, see Heijltjes, Van Gog, Leppink, and Paas (2014, p. 40) and Wasserman, Dorner, and Kao (1990).

\subsection{QUESTIONNAIRE ATIIUDES TOWARDS TEACHING CRITICAL THINKING}

We intended to measure attitudes towards teaching critical thinking with a 16-item questionnaire that addressed perceived relevance with 4 items and perceived competence with three underlying subscales: perceived competence regarding own critical thinking skills (4 items), the ability to recognize students' reasoning biases (4 items), and instructing critical thinking skills to students (4 items). However, because this factor structure did not fit our data well, we explored alternative factor structures in the pretest data. We found that a two-factor model with a total of 6 items fitted the data best. Important revisions were that we excluded the competence items addressing teachers' own critical thinking skills (as this did not concern teaching) and the items that lacked a reference to a specific activity related to teaching critical thinking (e.g., "I am able to integrate critical thinking in the content I am teaching"). Two confirmative factor analyses on the intermediate test and posttest data confirmed this factor structure. Therefore, we reduced the 16 -item version to a 6 -item version as mentioned in the manuscript:

\section{Perceived relevance (translated from Dutch):}

1. Critical thinking during educational activities encourages students to become independent thinkers.

2. Learning outcomes will improve from critical thinking during educational activities.

3. Critical thinking allows students to better understand the course content.

\section{Perceived competence (translated from Dutch):}

4. I notice it immediately when students commit a thinking fallacy during my lessons.

5. I can explain clearly to my students how they are drawing incorrect conclusions from the available information.

6. I can explain various fallacies to my students in such a way that they understand it. 


\subsection{SUPPLEMENTARY RESULTS}

Table S3.1. Means (M) and standard deviations (SD) of performance on heuristics-andbiases tasks per task category at pretest, intermediate test, and posttest per condition

\begin{tabular}{|c|c|c|c|c|}
\hline & \multicolumn{2}{|c|}{ Control } & \multicolumn{2}{|c|}{ Training } \\
\hline & M & SD & $M$ & SD \\
\hline \multicolumn{5}{|c|}{ Syllogisms with belief bias (range: $0-7$ ) } \\
\hline Pretest & 4.5 & 1.4 & 4.5 & 1.0 \\
\hline Intermediate test & 4.5 & 1.5 & 5.3 & 1.2 \\
\hline Posttest & 4.2 & 1.2 & 4.9 & 1.2 \\
\hline \multicolumn{5}{|c|}{ Base-rate tasks (range: 0-3) } \\
\hline Pretest & 1.3 & 1.0 & 1.8 & 0.8 \\
\hline Intermediate test & 1.5 & 0.7 & 2.2 & 0.6 \\
\hline Posttest & 1.4 & 0.8 & 2.3 & 0.8 \\
\hline \multicolumn{5}{|c|}{ Wason selection tasks (range: $0-3$ ) } \\
\hline Pretest & 0.3 & 0.6 & 0.3 & 0.6 \\
\hline Intermediate test & 0.6 & 0.8 & 0.7 & 1.0 \\
\hline Posttest & 0.5 & 0.8 & 0.6 & 1.0 \\
\hline \multicolumn{5}{|c|}{ Covariation detection tasks (range: $0-2$ ) } \\
\hline Pretest & 1.8 & 0.4 & 1.5 & 0.7 \\
\hline Intermediate test & 1.8 & 0.5 & 1.6 & 0.6 \\
\hline Posttest & 1.8 & 0.4 & 1.3 & 0.8 \\
\hline
\end{tabular}


Table S3.2. Percentage of teachers who detected and correctly explained the hidden biases in the vignettes (for each bias separately) at pretest, intermediate test, and posttest per condition

\begin{tabular}{|c|c|c|c|c|}
\hline & \multicolumn{2}{|c|}{ Bias detection } & \multicolumn{2}{|c|}{ Bias explanation } \\
\hline & Control & Training & Control & Training \\
\hline \multicolumn{5}{|c|}{ Belief bias (denial of the antecedent) } \\
\hline Pretest & $68.2 \%$ & $59.4 \%$ & $36.4 \%$ & $25.0 \%$ \\
\hline Intermediate test & $50.0 \%$ & $50.0 \%$ & $22.7 \%$ & $25.0 \%$ \\
\hline Posttest & $52.6 \%$ & $80.0 \%$ & $5.3 \%$ & $46.7 \%$ \\
\hline \multicolumn{5}{|c|}{ Belief bias (affirmation of the consequent) } \\
\hline Pretest & $63.6 \%$ & $84.4 \%$ & $45.5 \%$ & $50.0 \%$ \\
\hline Intermediate test & $36.4 \%$ & $59.4 \%$ & $4.5 \%$ & $12.5 \%$ \\
\hline Posttest & $73.7 \%$ & $93.3 \%$ & $31.6 \%$ & $50.0 \%$ \\
\hline \multicolumn{5}{|c|}{ Confirmation bias (Wason selection task) } \\
\hline Pretest & $36.4 \%$ & $34.4 \%$ & $13.6 \%$ & $6.3 \%$ \\
\hline Intermediate test & $13.6 \%$ & $40.6 \%$ & $0.0 \%$ & $3.1 \%$ \\
\hline Posttest & $47.4 \%$ & $96.7 \%$ & $5.3 \%$ & $16.7 \%$ \\
\hline \multicolumn{5}{|l|}{ Base-rate neglect bias } \\
\hline Pretest & $31.8 \%$ & $28.1 \%$ & $9.1 \%$ & $9.4 \%$ \\
\hline Intermediate test & $50.0 \%$ & $53.1 \%$ & $4.5 \%$ & $12.5 \%$ \\
\hline Posttest & $36.8 \%$ & $93.3 \%$ & $0.0 \%$ & $36.7 \%$ \\
\hline \multicolumn{5}{|c|}{ Covariation detection problem } \\
\hline Pretest & $54.5 \%$ & $75.0 \%$ & $22.7 \%$ & $18.8 \%$ \\
\hline Intermediate test & $9.1 \%$ & $34.4 \%$ & $0.0 \%$ & $15.6 \%$ \\
\hline Posttest & $73.7 \%$ & $93.3 \%$ & $15.8 \%$ & $33.3 \%$ \\
\hline
\end{tabular}


Table S3.3. Effect of critical thinking training on teachers' invested mental effort in learning and transfer (heuristics-and-biases) tasks

\begin{tabular}{|c|c|c|c|}
\hline & $\begin{array}{c}\mathrm{M} 1: \\
\text { intercept only }\end{array}$ & $\begin{array}{c}\text { M2: } \\
\text { effect occasion }\end{array}$ & $\begin{array}{c}\text { M3: } \\
\text { effect of training }\end{array}$ \\
\hline \multicolumn{4}{|l|}{ Mental effort learning tasks } \\
\hline Fixed part & Coefficient (s.e.) & Coefficient (s.e.) & Coefficient (s.e.) \\
\hline Intercept & $4.28(0.13)^{\star \star}$ & $4.09(0.15)^{\star \star}$ & $3.91(0.23)^{\star \star}$ \\
\hline Pre-Intermediate & & $0.48(0.12)^{\star \star}$ & $0.28(0.19)$ \\
\hline Pre-Post & & $0.06(0.12)$ & $0.02(0.20)$ \\
\hline Condition & & & $0.30(0.30)$ \\
\hline Pre-Intermediate $\times$ Condition & & & $0.34(0.24)$ \\
\hline Pre-Post $\times$ Condition & & & $0.06(0.25)$ \\
\hline \multicolumn{4}{|l|}{ Random part } \\
\hline Occasion and error variance $\left(\sigma_{e}^{2}\right)$ & $0.46(0.68)$ & $0.39(0.63)$ & $0.38(0.62)$ \\
\hline Teacher variance $\left(\sigma^{2} u 0\right)$ & $0.79(0.89)$ & $0.81(0.90)$ & $0.77(0.88)$ \\
\hline Deviance & 420.3 & 403.2 & 398.2 \\
\hline \multicolumn{4}{|l|}{ Mental effort transfer tasks } \\
\hline Fixed Part & Coefficient (s.e.) & Coefficient (s.e.) & Coefficient (s.e.) \\
\hline Intercept & $5.17(0.18)^{\star \star}$ & $5.12(0.20)^{\star \star}$ & $5.27(0.31)^{\star \star}$ \\
\hline Pre-Intermediate & & $0.20(0.16)$ & $-0.23(0.23)$ \\
\hline Pre-Post & & $-0.06(0.17)$ & $-0.99(0.24)$ \\
\hline Condition & & & $-0.25(0.40)$ \\
\hline Pre-Intermediate $\times$ Condition & & & $0.71(0.30)^{\star}$ \\
\hline Pre-Post $\times$ Condition & & & $1.51(0.31)^{\star \star}$ \\
\hline \multicolumn{4}{|l|}{ Random part } \\
\hline Occasion and error variance $\left(\sigma^{2} e\right)$ & $0.73(0.86)$ & $0.72(0.85)$ & $0.58(0.76)$ \\
\hline Teacher variance $\left(\sigma^{2} u 0\right)$ & $1.50(1.22)$ & $1.50(1.23)$ & $1.50(1.23)$ \\
\hline Deviance & 501.4 & 498.7 & 475.9 \\
\hline
\end{tabular}

Note. Occasion is dummy-coded in two dummies (Intermediate test, Posttest) with Pretest as reference category. Condition coded $0=$ control, $1=$ training. ${ }^{*} p<.05,{ }^{* \star} p<.001$.

\section{SUPPLEMENTARY MATERIALS - CHAPTER 4}

\subsection{CRITICAL THINKING TRAINING}

As noted, the goal of the interactive CT-training was to introduce the concept biased reasoning and to practice with avoiding bias in teachers' own thinking and reasoning. Teachers were trained in avoiding belief bias in syllogistic reasoning (i.e., tendency to be influenced by the believability of a conclusion when evaluating the logical validity of arguments, see 
e.g., Markovits \& Nantel, 1989; Newstead, Pollard, Evans, \& Allen, 1992) and in avoiding confirmation bias in Wason selection tasks (tendency to verify rules/hypotheses rather than to falsify them, see e.g., Dawson, Gilovich, \& Regan, 2002; Nickerson, 1998).

First, we started with a general introduction on $\mathrm{CT}$ and its relation with heuristics and biases. Second, we explained the definition of a logically valid argument and four basic laws of logic (see e.g., Evans, 2002): (1) modus ponens (if p then q, p therefore q; valid); (2) denial of the antecedent (if $p$ then $q$, not $p$ therefore not q; invalid); (3) affirmation of the consequent (if $\mathrm{p}$ then $\mathrm{q}, \mathrm{q}$ therefore $\mathrm{p}$; invalid); and (4) modus tollens (if $\mathrm{p}$ then $\mathrm{q}$, not $\mathrm{q}$ therefore not $\mathrm{p}$; valid). We demonstrated belief bias in each of the four laws by contrasting a syllogism without belief bias (e.g., if someone is a teacher then this person has a job, John is not a teacher therefore John does not have a job [cf. denial of the antecedent]) to a syllogism with belief bias (e.g., if a DNA test prediction matches with actual health outcomes then the test is valuable, the DNA test prediction for heart failure does not match with actual health outcomes therefore the DNA test for heart failure is not valuable [cf. denial of the antecedent]). Teachers could respond whether or not the conclusions were correct according to them, hereafter we explained the correct answers using worked examples. Third, teachers could practice the laws of logic with eight (belief-biased) syllogistic reasoning problems. Fourth, we ended the training with solving two Wason selection tasks together (one abstract version and one with contextual information, see Cosmides, 1989). An example of the abstract version is:

Each of the four cards below have an image on one side and a number on the other side. The following rule applies to the cards:

'If there is a clover on one side, then there is a 9 on the other side'.

Choose those cards and only those cards that need to be turned over in order to decide whether the rule is true or false (correct answer: clover and 2).

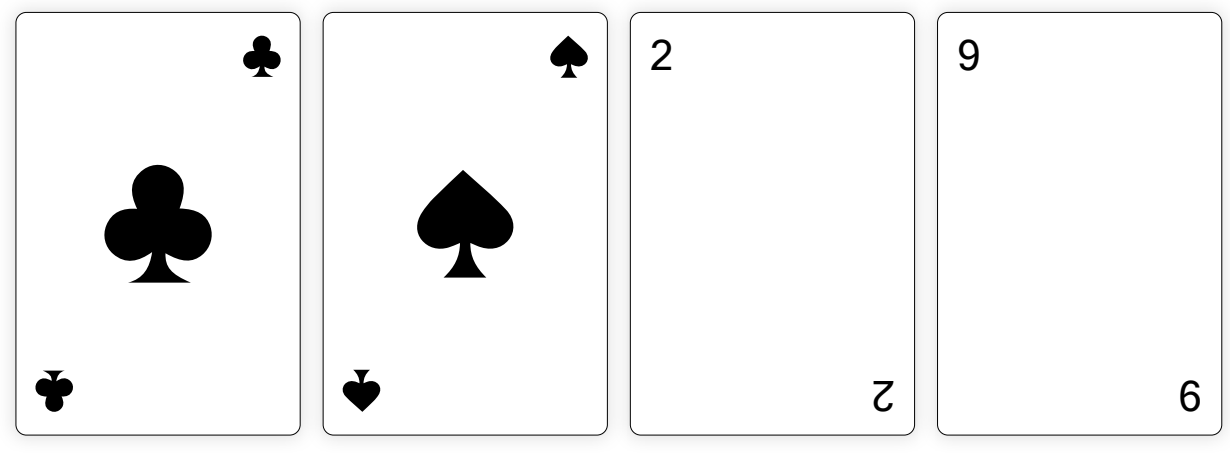

The goal was to show how the same laws of logic also apply (next to syllogistic reasoning) to reasoning about the verification of rules/hypotheses and how confirmation bias can cause 
individuals' tendency to verify hypotheses incorrectly with if $\mathrm{p}$ then $\mathrm{q}, \mathrm{q}$ therefore $\mathrm{p}$ (cf. affirmation of the consequent) rather than to falsify correctly with if $p$ then $q$, not $q$ therefore not p (cf. modus tollens, see also Evans, 2002).

\subsection{FULL TEST INSTRUCTION}

You will be presented a total of 7 short texts that were part of various group assignments written by Safety-and-Security-Management students. Each text contains an argument that is logically invalid. After each text, please explain these students why their argument is invalid and how they can improve their argument.

A logically invalid argument is an argument where a drawn conclusion does not necessarily follow from the given information or claims in a text. For this assignment, you do not need to assess whether the preceding information in the texts is reliable or true (assume that all information provided in the texts is true); you only need to explain why the students' conclusion cannot be drawn from the information provided in the text. Remember to address your explanation to students, so make your explanation as concrete as possible and target your explanation to the specific argument in the students' text.

You may use scrap paper during this assignment, but you are not supposed to search for additional information on the Internet.

\subsection{EXAMPLE BIAS EXPLANATION FOR TASK EXAMPLE IN FIGURE 4.2 (CF. DENIAL OF THE ANTECEDENT)}

You cannot know for sure that the port does not sufficiently minimize the risk of incidents with harmful consequences (detection). The IMO only tells you something about the consequences of following the SOLAS guidelines and not about not following these guidelines (information-lack explanation). It could be the case that the Port of Rotterdam has its own safety policy beyond the SOLAS guidelines that also sufficiently minimizes the risk of incidents with harmful consequences (counter-example explanation). You can improve your argument by placing a reservation to the conclusion. For example: "Because a recent inspection investigation shows that the Port of Rotterdam does not follow all the SOLAS guidelines, it may be possible that the port does not sufficiently minimize the risk of incidents with harmful consequences" (reservation suggestion). Your original conclusion would be correct if you would have specific information about the consequences of not following all SOLAS guidelines on the port's risk of incidents with harmful consequences (required-information suggestion). 


\subsection{TRANSLATED TASK EXAMPLES WITH EXAMPLE EXPLANATIONS TO STUDENTS}

Task example with an affirmation-of-the-consequent fallacy in students' syllogistic reasoning (learning task):

\section{Students wrote the following in a group assignment where they advised the Municipality of Moerdijk about the placement of a wind turbine}

The distribution of danger zones consists of six zones. The largest zone is Zone 1. When devices fall under high-risk establishments (for example, due to chemical and heavy industrial activities), they must be in Zone 1 (Environmental Service, 2017). At this moment, the old $3 \mathrm{MW}$ wind turbine must be in Zone 1. We can therefore assume that it concerns a high-risk establishment.

Explain students why their argument is invalid.

Explain students how they could improve their argument.

Many people tend to agree with the conclusion that the $3 \mathrm{MW}$ wind turbine must be in Zone 1. However, this is not logically correct because the line of reasoning 'if $p$ then $q, q$ therefore p' is not valid (cf. affirmation of the consequent). A possible explanation to students could be:

You cannot know for sure that the $3 \mathrm{MW}$ wind turbine concerns a high-risk establishment (detection). The Environmental Service only tells you that all high-risk establishments must be in Zone 1, not that all devices in Zone 1 are high-risk establishments (information-lack explanation). It could be the case that there is an additional rule which states that all wind turbines must be in Zone 1, independent of falling under high-risk establishments or not (counter-example explanation). You can improve your argument by placing a reservation to the conclusion. For example: "The 3MW wind turbine must currently be in Zone 1. It may therefore be possible that it concerns a high-risk establishment" (reservation suggestion). Your original conclusion would be correct if you would have specific information about the reason why the $3 \mathrm{MW}$ wind turbine must be in Zone 1 (required-information suggestion).

Task example with an affirmation-of-the-consequent fallacy in students' reasoning about hypothesis verification (cf. Wason selection task; transfer task): 


\section{Students wrote the following in a group assignment where they were instructed to create a safe living environment for care home "Fort Isabella":}

Cameras work preventively against intruders. It is claimed that placing cameras in front of entrances to health care institutions greatly reduces the number of sneak-in attempts. To investigate this claim in practice, we will conduct an interview with the managers of the healthcare institutions Horizon and PlusZorg. For the past two years, Horizon has installed cameras in front of the entrances to all its care homes. We ask the health care manager whether the number of sneak-in attempts has decreased since the placement of the cameras. At PlusZorg, the number of sneakin attempts has fallen dramatically since four years. We are very curious if this was the result of installing cameras. With both interviews we can test whether the abovementioned claim also applies to the two care homes.

Explain students why their argument is invalid.

Explain students how they could improve their argument.

Many people tend to agree with the conclusion that both interviews can verify whether the claim applied to the two care homes. Although this is logically correct for Horizon, it not correct for PlusZorg because verifying a hypothesis with the line of reasoning if $p$ then $\mathrm{q}$, q therefore p' is not valid (cf. affirmation of the consequent). The claim could instead be correctly falsified with 'with if $p$ then $q$, $-q$ therefore $-p$ (cf. modus tollens). A possible explanation to students could be:

You cannot verify for sure whether the claim applies to PlusZorg (detection). The claim tells you something about the consequences of placing cameras and not about causes of reduced numbers of sneak-in attempts (information-lack explanation). It could be the case that PlusZorg implemented something else that caused the decreased sneak-in attempts. In this scenario, it can still be the case that placing cameras also reduces the number of sneak-in attempts for PlusZorg. You can improve your argument by placing a reservation to the conclusion. For example: "We are curious whether PlusZorg's decreased number of sneak-in attempts was the result of placing cameras. This way can possibly find out whether the claim also applied to PlusZorg" (reservation suggestion). Your original conclusion would be correct if the second interview was conducted with a manager of a care home with an increased number of sneak-in attempts and you asked him/her about the placement of cameras. 


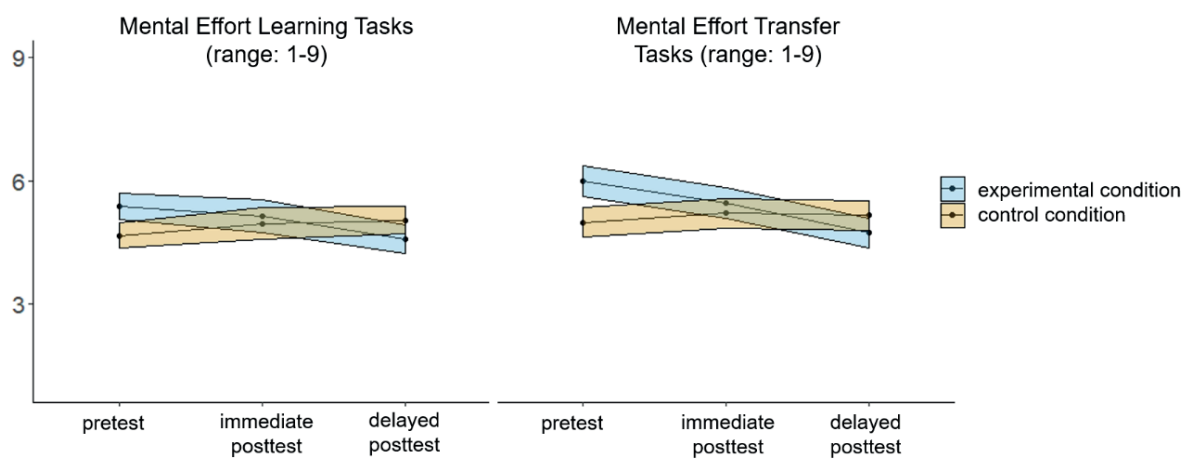

Figure S4.1. Plotted interaction effects between Test Occasion and Condition. Error bands indicate standard error.

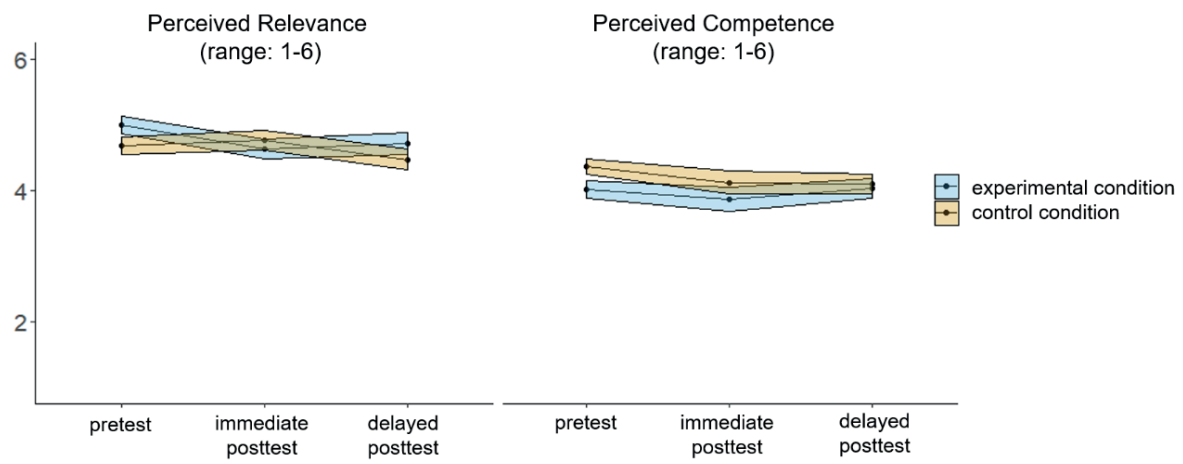

Figure S4.2. Plotted interaction effects between Test Occasion and Condition. Error bands indicate standard error. 
Table S4.1. Overview of coding scheme with coded examples (translated from dutch) belonging to the task shown in Section 4 of the Supplementary Materials (cf. affirmation of the consequent)

\section{Explain students why their argument is invalid}

\begin{tabular}{|c|c|c|}
\hline Code & Definition & Coded example \\
\hline no explanation (0p) & $\begin{array}{l}\text { The explanation does not contain } \\
\text { any (correct) statements about } \\
\text { logic of the students' argument }\end{array}$ & "I do not understand this argument." \\
\hline detection (1p) & $\begin{array}{l}\text { The explanation shows } \\
\text { recognition of the invalid } \\
\text { conclusion }\end{array}$ & $\begin{array}{l}\text { "The fact that the old } 3 \mathrm{MW} \text { wind } \\
\text { turbine is in Zone } 1 \text { does not mean that } \\
\text { it is actually a high-risk establishment." }\end{array}$ \\
\hline $\begin{array}{l}\text { information-lack } \\
\text { explanation (2p) }\end{array}$ & $\begin{array}{l}\text { The explanation points out what } \\
\text { necessary information is lacking } \\
\text { to draw the students' conclusion }\end{array}$ & $\begin{array}{l}\text { "Why does the old } 3 \mathrm{MW} \text { wind turbine } \\
\text { have to be in zone } 1 \text { right now, that is } \\
\text { not clear to me..." }\end{array}$ \\
\hline $\begin{array}{l}\text { counter-example } \\
\text { explanation }(3 p)\end{array}$ & $\begin{array}{l}\text { The explanation demonstrates } \\
\text { through a counter example how } \\
\text { the students' argument can also } \\
\text { yield a different conclusion }\end{array}$ & $\begin{array}{l}\text { "If all devices with risks are in zone } 1 \text {, } \\
\text { does this mean that conversely in zone } \\
1 \text { only devices with risks can be found? } \\
\text { Can there also be other reasons for } \\
\text { placing a device in zone } 1 \text { ?" }\end{array}$ \\
\hline
\end{tabular}

Explain students how they could improve their argument

\begin{tabular}{|c|c|c|}
\hline Code & Definition & Coded example \\
\hline no suggestion (0p) & $\begin{array}{l}\text { The explanation does not contain } \\
\text { concrete (correct) suggestions to } \\
\text { improve the logic of the students' } \\
\text { argument }\end{array}$ & $\begin{array}{l}\text { "In this case I would ask more } \\
\text { explanation from the students on this } \\
\text { text." }\end{array}$ \\
\hline $\begin{array}{l}\text { reservation } \\
\text { suggestion (1p) }\end{array}$ & $\begin{array}{l}\text { The explanation suggests to } \\
\text { place a reservation to the } \\
\text { conclusion }\end{array}$ & "make a reservation in the argument." \\
\hline $\begin{array}{l}\text { required-information } \\
\text { suggestion }(2 p)\end{array}$ & $\begin{array}{l}\text { The explanation provides a } \\
\text { suggestion on concrete } \\
\text { information that would allow the } \\
\text { students to draw their original } \\
\text { conclusion }\end{array}$ & $\begin{array}{l}\text { "...Test the device following the high- } \\
\text { risk criteria and not based on where it } \\
\text { is located. I would think of another } \\
\text { example to explain to them..." }\end{array}$ \\
\hline
\end{tabular}

Note. For conciseness we did not copy the full explanations to the 'Coded example' column, only the phrases that were illustrative of the code definition. 
Table S4.2. Overview of coding scheme with coded examples (translated from dutch) belonging to the task shown in section 4 of the supplementary materials (cf. wason selection task)

\section{Explain students why their argument is invalid}

\begin{tabular}{ll} 
Code & Definition \\
\hline no explanation (0p) & $\begin{array}{l}\text { The explanation does not } \\
\text { contain concrete (correct) } \\
\text { suggestions to improve the logic } \\
\text { of the students' argument } \\
\text { The explanation shows } \\
\text { recognition of the invalid } \\
\text { conclusion }\end{array}$ \\
detection (1p) & $\begin{array}{l}\text { The explanation points out what } \\
\text { necessary information is lacking } \\
\text { to draw the students' conclusion } \\
\text { explanation (2p) }\end{array}$ \\
& $\begin{array}{l}\text { The explanation demonstrates } \\
\text { through a counter example how } \\
\text { the students' argument can also } \\
\text { yield a different conclusion }\end{array}$ \\
\end{tabular}

\section{Coded example}

"You want to investigate a claim, here while you are only going to interview two healthcare administrators..."

“... The interview with Horizon is in line with this reasoning, but the interview with PlusZorg is not."

"... For Horizon you can investigate this claim. For Pluszorg you do not know if they have cameras."

"Part 1: the already hung cameras lead to fewer intrusions; if yes from the interview, the statement is correct. Part 2: there are decreases at the other healthcare institution, if one has hung cameras there, the conclusion does not have to be logical; the decrease may also be due to other causes and / or no cameras may even have been hung."

\section{Explain students how they could improve their argument}

\begin{tabular}{ll} 
Code & Definition \\
\hline & The explanation does not \\
& contain any (correct)
\end{tabular}

no suggestion $(0 p)$

reservation

suggestion (1p)

required-information suggestion $(2 p)$

\begin{abstract}
suggestions to improve the logic of the students' argument
\end{abstract}

The explanation suggests to place a reservation to the conclusion

The explanation provides a suggestion on concrete information that would allow the students to draw their original conclusion
Coded example

"also ask the question if more effort has been made."

\begin{abstract}
"... include a reservation. We are not sure why the number of sneak-in attempts has fallen."

"Focus your research on healthcare institutions that have not had a decrease in the number of incidents; if they have hung cameras, the claim is incorrect: healthcare institutions that have hung cameras, if they have no decrease in the number of incidents, the claim is also incorrect. If they do have a decrease, that is proof that the claim for those care homes is valid.
\end{abstract}

Note. For conciseness we did not copy the full explanations to the 'Coded example' column, only the phrases that were illustrative of the code definition. 
Table S4.3. Two-way mixed, absolute agreement, single-measures intra-class correlations (ICC) for each explanation code category separately

\begin{tabular}{lccc}
\hline & Pretest & Immediate posttest & Delayed posttest \\
\hline Learning Tasks & & & \\
Explanation codes & .763 & .638 & .822 \\
$\quad$ Suggestion codes & .785 & .523 & .779 \\
$\quad$ Invalid logic & .847 & .487 & .907 \\
Transfer Tasks & & & \\
$\quad$ Explanation codes & .501 & .901 & .845 \\
Suggestion codes & 1.00 & 1.00 & .659 \\
Invalid logic & .642 & .642 & .843 \\
\hline
\end{tabular}

Table S4.4. Explanation code frequencies $(n)$ on Learning Tasks for the experimental condition $(n=18)$ and the control condition $(n=19)$

\begin{tabular}{|c|c|c|c|c|c|c|c|c|}
\hline & \multicolumn{4}{|c|}{ Experimental condition } & \multicolumn{4}{|c|}{ Control condition } \\
\hline & DA1 & DA2 & AC1 & AC2 & DA1 & DA2 & $\mathrm{AC} 1$ & AC2 \\
\hline \multicolumn{9}{|l|}{ Pretest } \\
\hline No explanation & 4 & 9 & 1 & 6 & 2 & 6 & 2 & 8 \\
\hline Detection & 3 & 2 & 7 & 3 & 5 & 3 & 6 & 6 \\
\hline Information-lack explanation & 0 & 0 & 3 & 3 & 0 & 0 & 4 & 0 \\
\hline Counter-example explanation & 11 & 7 & 7 & 6 & 12 & 10 & 7 & 5 \\
\hline \multicolumn{9}{|l|}{ Immediate posttest } \\
\hline No explanation & 3 & 3 & 1 & 4 & 6 & 6 & 3 & 3 \\
\hline Detection & 6 & 2 & 4 & 3 & 7 & 4 & 9 & 6 \\
\hline Information-lack explanation & 1 & 1 & 3 & 0 & 1 & 1 & 1 & 1 \\
\hline Counter-example explanation & 8 & 12 & 10 & 11 & 5 & 8 & 6 & 9 \\
\hline \multicolumn{9}{|l|}{ Delayed posttest } \\
\hline No explanation & 4 & 8 & 2 & 0 & 4 & 8 & 0 & 0 \\
\hline Detection & 5 & 1 & 1 & 1 & 3 & 3 & 5 & 4 \\
\hline Information-lack explanation & 0 & 1 & 5 & 0 & 0 & 0 & 4 & 1 \\
\hline Counter-example explanation & 9 & 8 & 10 & 17 & 12 & 8 & 10 & 14 \\
\hline
\end{tabular}

Note. DA1-DA2 $=$ denial of the antecedent fallacy task number 1 and 2. AC1-AC2 $=$ affirmation of the consequent fallacy task number 1 and 2 . 
Table S4.5. Suggestion code frequencies $(n)$ on Learning Tasks for the experimental condition $(n=18)$ and the control condition $(n=19)$

\begin{tabular}{|c|c|c|c|c|c|c|c|c|}
\hline & \multicolumn{4}{|c|}{ Experimental condition } & \multicolumn{4}{|c|}{ Control condition } \\
\hline & DA1 & DA2 & $\mathrm{AC} 1$ & AC2 & DA1 & DA2 & $\mathrm{AC} 1$ & AC2 \\
\hline \multicolumn{9}{|l|}{ Pretest } \\
\hline No suggestion & 14 & 10 & 12 & 6 & 12 & 8 & 12 & 10 \\
\hline Reservation suggestion & 1 & 2 & 0 & 0 & 1 & 3 & 2 & 0 \\
\hline Required-information suggestion & 3 & 6 & 6 & 12 & 6 & 8 & 5 & 9 \\
\hline \multicolumn{9}{|l|}{ Immediate posttest } \\
\hline No suggestion & 6 & 5 & 9 & 5 & 12 & 14 & 14 & 11 \\
\hline Reservation suggestion & 2 & 1 & 2 & 3 & 1 & 2 & 1 & 1 \\
\hline Required-information suggestion & 10 & 12 & 7 & 10 & 6 & 3 & 4 & 7 \\
\hline \multicolumn{9}{|l|}{ Delayed posttest } \\
\hline No suggestion & 12 & 10 & 4 & 2 & 12 & 11 & 7 & 8 \\
\hline Reservation suggestion & 2 & 2 & 3 & 3 & 2 & 2 & 1 & 2 \\
\hline Required-information suggestion & 4 & 6 & 11 & 13 & 5 & 6 & 11 & 9 \\
\hline
\end{tabular}

Note. DA1-DA2 $=$ denial of the antecedent fallacy task number 1 and 2. AC1-AC2 $=$ affirmation of the consequent fallacy task number 1 and 2.

Table S4.6. Explanation code frequencies $(n)$ on Transfer Tasks for the experimental condition $(n=18)$ and the control condition $(n=19)$

\begin{tabular}{|c|c|c|c|c|c|c|}
\hline & \multicolumn{3}{|c|}{ Experimental condition } & \multicolumn{3}{|c|}{ Control condition } \\
\hline & WS1 & WS2 & WS3 & WS1 & WS2 & WS3 \\
\hline \multicolumn{7}{|l|}{ Pretest } \\
\hline No explanation & 17 & 18 & 18 & 16 & 15 & 18 \\
\hline Detection & 0 & 0 & 0 & 0 & 0 & 0 \\
\hline Information-lack explanation & 1 & 0 & 0 & 1 & 1 & 0 \\
\hline Counter-example explanation & 0 & 0 & 0 & 2 & 3 & 1 \\
\hline \multicolumn{7}{|l|}{ Immediate posttest } \\
\hline No explanation & 12 & 11 & 9 & 14 & 11 & 17 \\
\hline Detection & 1 & 1 & 4 & 4 & 2 & 0 \\
\hline Information-lack explanation & 0 & 1 & 0 & 0 & 0 & 0 \\
\hline Counter-example explanation & 5 & 5 & 5 & 1 & 6 & 2 \\
\hline \multicolumn{7}{|l|}{ Delayed posttest } \\
\hline No explanation & 8 & 12 & 10 & 12 & 11 & 9 \\
\hline Detection & 0 & 1 & 1 & 1 & 2 & 2 \\
\hline Information-lack explanation & 3 & 1 & 2 & 1 & 0 & 2 \\
\hline Counter-example explanation & 7 & 4 & 5 & 5 & 6 & 6 \\
\hline
\end{tabular}

Note. WS1-WS3 = Wason selection task number 1-3. 
Table S4.7. Suggestion code frequencies $(n)$ on Transfer Tasks for the experimental condition $(n=18)$ and the control condition $(n=19)$

\begin{tabular}{|c|c|c|c|c|c|c|}
\hline & \multicolumn{3}{|c|}{ Experimental condition } & \multicolumn{3}{|c|}{ Control condition } \\
\hline & WS1 & WS2 & WS3 & WS1 & WS2 & WS3 \\
\hline \multicolumn{7}{|l|}{ Pretest } \\
\hline No suggestion & 17 & 18 & 18 & 19 & 19 & 19 \\
\hline Reservation suggestion & 0 & 0 & 0 & 0 & 0 & 0 \\
\hline Required-information suggestion & 1 & 0 & 0 & 0 & 0 & 0 \\
\hline \multicolumn{7}{|l|}{ Immediate posttest } \\
\hline No suggestion & 15 & 14 & 14 & 16 & 18 & 18 \\
\hline Reservation suggestion & 1 & 4 & 3 & 1 & 0 & 0 \\
\hline Required-information suggestion & 2 & 0 & 1 & 2 & 1 & 1 \\
\hline \multicolumn{7}{|l|}{ Delayed posttest } \\
\hline No suggestion & 15 & 16 & 15 & 18 & 17 & 18 \\
\hline Reservation suggestion & 2 & 1 & 2 & 0 & 1 & 0 \\
\hline Required-information suggestion & 1 & 1 & 1 & 1 & 1 & 1 \\
\hline
\end{tabular}

Note. WS1-WS3 = Wason selection task number 1-3.

\section{SUPPLEMENTARY MATERIALS - CHAPTER 5}

\subsection{CLASSICAL CONJUNCTION PROBLEM}

An example of a classical conjunction problem (see e.g., De Neys et al., 2011; Frey et al., 2018):

Jon is 32 . He is intelligent and punctual but unimaginative and somewhat lifeless. In school, he was strong in mathematics but weak in languages and art.

Which one of the following statements is most likely?

Jon plays in a rock band

Jon plays in a rock band and is an accountant

Because Jon's description is more representative of an accountant, most people incorrectly indicate that it is more likely that Jon plays in a rock band and is accountant than that Jon is a rock band player only. However, the conjunction of any two probabilities can never be more likely than either of the conjuncts in isolation. 


\subsection{ITEM-LEVEL CHECK}

In order to check whether the content of the items' cover stories influenced the accuracy of the items, we conducted a logistic mixed effect model, with response accuracy as dependent variable (incorrect $=0$; incorrect $=1$ ), item-number content as fixed effect and task format ( problem solving $=0$; vignette $=1)$, conflict version $($ conflict $=0$; no conflict $=1$ ), and participants' identification as random effects. Results of the model for the base-rate tasks, indicated that - after controlling for variance due to task format or conflict version - two items were performed significantly better compared to the other test items, $\mathrm{B}=0.65 \mathrm{SE}=$ $0.28, \mathrm{~W}=2.31, p=.021 ; \mathrm{B}=0.61 \mathrm{SE}=0.28, \mathrm{~W}=2.17, p=.030$. However, post-hoc pairwise comparisons between all items did not yield significant accuracy differences between any of the items ( $p s \geq .140)$. Thus, base-rate accuracy did not seem to depend on the content of the cover stories.

For the conjunction items, we found that six items were performed significantly better compared to the other test items ( $p \mathrm{~s} \leq .019)$, and that one item was performed significantly worse, $\mathrm{B}=0.70 \mathrm{SE}=0.28, \mathrm{~W}=-2.52, p=.012$. Post-hoc pairwise comparisons between all items showed no significant accuracy differences between items ( $p \mathrm{~s} \geq .095)$, except for the one item that was performed worse compared to other items $(p s \leq .010)$. Based on the content of the cover story, we could not directly explain the obtained accuracy difference. Because we only found one potentially different item, we decided not to exclude any items. Note that we counterbalanced the content of the reasoning tasks across task formats and conflict versions and always included item-content number as random effect in our statistical models to account for potential variance due to the item's content. Hence, a difference in one item is unlikely to affect our conclusions.

\subsection{INDIVIDUAL DIFFERENCES IN CONFLICT DETECTION}

Next to investigating whether conflict detection takes place at the group level, we explored potential individual differences in conflict detection and tested the consistency of conflict detection across task formats.

\section{BASE-RATE TASKS}

Consistent with the manuscript structure, we first report all results for the base-rate tasks and then for the conjunction tasks.

Number of detectors. We analyzed how many of the biased reasoners showed conflict detection. For both task formats, we tallied per conflict detection index which percentage of the biased reasoners showed the conflict detection effect, a reversed conflict detection effect, or no effect (no difference between error measures on conflict and no-conflict trials). The results are shown in Table S1 (top two panels). 
Confidence (\%). For the problem-solving tasks, we found that $65.9 \%$ of the biased reasoners showed conflict detection as indexed by their confidence ratings, with an average effect size of $-18.1 \%(\mathrm{SD}=16.2)$. This was rather similar on the vignette tasks, with the majority $(65.3 \%)$ of the biased reasoners showing conflict detection, with an average effect size of $-17.5 \%$ ( $\mathrm{SD}=16.6)$. Both the percentage of conflict detectors and the size of the effect were comparable to previous findings of Frey et al. (2018) on classical base-rate tasks, who found that $72 \%$ of the biased participants were conflict detectors with a confidence effect size of $-20.0 \%$.

Response time (s). For the response time index, we found that $51.1 \%$ of the biased participants showed conflict detection on the problem-solving tasks, with an average conflict detection effect size of $9.9 \mathrm{~s}(\mathrm{SD}=18.7)$. Likewise, $50.7 \%$ of the biased participants showed conflict detection on the vignette tasks, with an average size of $12.5 \mathrm{~s}$ (SD =15.8). These findings deviated from Frey et al. (2018) who found a slightly larger group of conflict detectors $(64 \%)$, yet with a smaller effect size $(\mathrm{M}=4.2 \mathrm{~s})$.

Confidence response time (s). For the problem-solving tasks, we found that $60.7 \%$ of the biased participants showed conflict detection on the confidence response time index, with an average effect size $0.9 \mathrm{~s}(\mathrm{SD}=1.4)$. For the vignette tasks we found that $54.9 \%$ showed conflict detection, with an effect size of $1.1 \mathrm{~s}(\mathrm{SD}=1.5)$. These findings were more or less in line with Frey et al. (2018), who found that somewhat smaller group of biased participants showed conflict detection (43\%), yet with a comparable effect size ( $\mathrm{M}=1.3 \mathrm{~s})$.

In sum, even when taking individual differences into account, we found very similar conflict detection patterns across both base-rate task formats (problem-solving tasks and vignette tasks). Furthermore, apart from some small differences, we found similar conflict detection patterns on the current, more realistic versions of base-rate tasks as Frey et al. (2018) found with classical base-rate problems.

Accuracy correlations. We calculated the correlation between each individual's conflict detection effect size on the three conflict detection indices and their total accuracy on the conflict tasks. The results are also included in Table S1 (top two panels). Results showed only one significant correlation for the base-rate problem-solving tasks. Within the reversed detection group, a larger response time effect size was related to lower accuracy on conflict tasks, $\mathrm{r}=-0.32, p=.009$. Hence, the longer participants took to answer correctly solved no-conflict tasks (relative to their incorrectly solved conflict tasks), the more likely it was that they solved other conflict tasks incorrectly. For the vignette tasks, the analyses yielded two significant correlations, both within the detection group. A larger confidence effect size, $\mathrm{r}=-0.24, p=.022$, and a larger response-time effect size, $\mathrm{r}=0.30, p=.010$, was related to higher total accuracy on conflict tasks. Hence, a lower confidence and longer response time on incorrectly evaluated decisions on conflict tasks (relative to correctly evaluated decision on no-conflict tasks) was associated with better accuracy on other conflict tasks. For the remaining conflict detection indices and subgroups the results are less clear, with only small and non-significant correlations. Hence, in these cases there is no clear evidence that the size of the conflict detection effect reflects individual differences in the quality of the detection process among biased reasoners. 
Table S5.1. Individual-level findings for different subgroups of biased reasoners and for the whole group of biased reasoners

\begin{tabular}{|c|c|c|c|c|}
\hline & $\begin{array}{c}\text { Subgroup: } \\
\text { Conflict } \\
\text { detection }\end{array}$ & $\begin{array}{l}\text { Subgroup: } \\
\text { Reversed } \\
\text { detection }\end{array}$ & $\begin{array}{l}\text { Subgroup: } \\
\text { Same }\end{array}$ & $\begin{array}{c}\text { Whole biased } \\
\text { group }\end{array}$ \\
\hline \multicolumn{5}{|l|}{ Base-rate problems } \\
\hline \multicolumn{5}{|l|}{ Confidence } \\
\hline$\%$ of biased group & $65.9(n=89)$ & $25.9(n=35)$ & $8.1(n=11)$ & $100(n=135)$ \\
\hline Conf. effect size (SD) & $-18.1(16.2)$ & $9.9(8.6)$ & - & $-9.4(18.6)$ \\
\hline Effect size-accuracy $r(p)$ & $-0.12(.250)$ & $0.19(.270)$ & - & $0.04(.700)$ \\
\hline \multicolumn{5}{|l|}{ Response time } \\
\hline$\%$ of biased group & $51.1(n=69)$ & $48.9(n=66)$ & - & $100(n=135)$ \\
\hline RT effect size $(S D)$ & $9.9(18.7)$ & $-8.8(13.0)$ & - & $0.7(18.6)$ \\
\hline Effect size-accuracy $r(p)$ & $0.23(.054)$ & $-0.32(.009)$ & - & $.008(.900)$ \\
\hline \multicolumn{5}{|l|}{ Confidence response time } \\
\hline$\%$ of biased group & $61.5(n=83)$ & $38.5(n=52)$ & - & $100(n=135)$ \\
\hline Conf. RT effect size $(S D)$ & $0.9(1.4)$ & $-0.5(0.6)$ & - & $0.3(1.3)$ \\
\hline Effect size-accuracy $r(p)$ & $0.10(.390)$ & $-0.05(.700)$ & & $-0.02(.800)$ \\
\hline \multicolumn{5}{|l|}{ Base-rate vignettes } \\
\hline \multicolumn{5}{|l|}{ Confidence } \\
\hline$\%$ of biased group & $65.3(n=94)$ & $25.7(n=37)$ & $9.0(n=13)$ & $100(n=144)$ \\
\hline Conf. effect size $(S D)$ & $-17.5(16.6)$ & $9.8(7.5)$ & 0 & $-8.9(18.4)$ \\
\hline Effect size-accuracy $r(p)$ & $-0.24(.022)$ & $0.19(.267)$ & - & $-0.11(.200)$ \\
\hline \multicolumn{5}{|l|}{ Response time } \\
\hline$\%$ of biased group & $50.7(n=70)$ & $49.3(n=74)$ & - & $100(n=144)$ \\
\hline $\mathrm{RT}$ effect size $(S D)$ & $12.5(15.8)$ & $-12.92(15)$ & - & $-0.6(19.9)$ \\
\hline Effect size-accuracy $r(p)$ & $0.30(.010)$ & $-0.21(.085)$ & - & $0.11(.200)$ \\
\hline \multicolumn{5}{|l|}{ Confidence response time } \\
\hline$\%$ of biased group & $51.4(n=74)$ & $47.9(n=69)$ & $0.7(n=1)$ & $100(n=144)$ \\
\hline Conf. RT effect size $(S D)$ & $1.1(1.5)$ & $-0.7(1)$ & 0 & $0.2(1.6)$ \\
\hline Effect size-accuracy $r(p)$ & $0.08(.470)$ & $-0.08(.550)$ & - & $0.1(.200)$ \\
\hline \multicolumn{5}{|l|}{ Conjunction problems } \\
\hline \multicolumn{5}{|l|}{ Confidence } \\
\hline$\%$ of biased group & $72.0(n=103)$ & $22.4(n=32)$ & $5.6(n=8)$ & $100(n=143)$ \\
\hline Conf. effect size $(S D)$ & $-16.0(12.3)$ & $6.4(4.8)$ & 0 & $-10.1(14.3)$ \\
\hline Effect size-accuracy $r(p)$ & $-0.35(<.001)$ & $0.09(.610)$ & - & $-0.30(<.001)$ \\
\hline \multicolumn{5}{|l|}{ Response time } \\
\hline$\%$ of biased group & $55.9(n=80)$ & $44.1(n=63)$ & - & $100(n=143)$ \\
\hline RT effect size $(S D)$ & $9.9(14.1)$ & $-8.7(9.7)$ & - & $1.7(15.4)$ \\
\hline Effect size-accuracy $r(p)$ & $0.30(.008)$ & $0.01(.925)$ & - & $0.24(.004)$ \\
\hline \multicolumn{5}{|l|}{ Confidence response time } \\
\hline$\%$ of biased group & $44.8(n=62)$ & $55.2(n=81)$ & - & $100(n=143)$ \\
\hline Conf. RT effect size (SD) & $0.6(0.7)$ & $-0.7(0.9)$ & - & $-0.1(1.0)$ \\
\hline Effect size-accuracy $r(p)$ & $0.16(.200)$ & $0.08(.490)$ & - & $0.04(.600)$ \\
\hline \multirow{2}{*}{\multicolumn{5}{|c|}{$\begin{array}{l}\text { Conjunction vignettes } \\
\text { Confidence }\end{array}$}} \\
\hline Confidence & & & & \\
\hline$\%$ of biased group & $57.9(n=84)$ & $37.2(n=54)$ & $4.8(n=7)$ & $100(n=145)$ \\
\hline Conf. effect size $(S D)$ & $-16.2(11.9)$ & $11.1(8.4)$ & & $-5.2(16.7)$ \\
\hline Effect size-accuracy $r(p)$ & $-0.29(.008)$ & $0.28(.041)$ & & $-0.16(.060)$ \\
\hline \multicolumn{5}{|l|}{ Response time } \\
\hline$\%$ of biased group & $54.5(n=79)$ & $45.5(n=66)$ & - & $100(n=145)$ \\
\hline RT effect size $(S D)$ & $9.2(7.3)$ & $-10.3(16.8)$ & & $0.4(15.8)$ \\
\hline Effect size-accuracy $r(p)$ & $0.07(.550)$ & $-0.13(.290)$ & & $0.04(.600)$ \\
\hline \multicolumn{5}{|l|}{ Confidence response time } \\
\hline$\%$ of biased group & $41.4(n=60)$ & $58.6(n=85)$ & - & $100(n=145)$ \\
\hline Conf. RT effect size $(S D)$ & $0.8(1.2)$ & $-1.1(3.4)$ & & $-0.3(2.8)$ \\
\hline Effect size-accuracy $r(p)$ & $0.29(.022)$ & $0.02(.886)$ & & $0.17(.050)$ \\
\hline
\end{tabular}

Note . Conf. $=$ confidence, $\mathrm{RT}=$ response time, $r=$ correlation coefficient. 
In sum, for the vignette tasks we found indications that the size of the conflict detection effects reflected individual differences in the quality of the detection process among biased reasoners. In line with Frey et al. (2018) for classical base-rate tasks, we obtained significant correlations on the confidence and response time indices. For problem-solving tasks, on the other hand, we found no such correlations.

Conflict detection consistency. Given the similarity of conflict detection patterns across both task formats, one would expect that individuals who detected conflict in problem-solving tasks, would also detect conflict in vignette tasks. Therefore, we used cross-tables to count how many of the biased participants showed conflict detection across both task formats. Results are shown in Tables S2-S4.

Confidence. Table S2 shows that $78.6 \%$ of all participants $(n=125$ out of 159$)$ were consistent biased reasoners (i.e., entering at least one biased response on one of the three conflict tasks in both task formats). Of these consistent biased reasoners, $42.4 \%$ was also a consistent conflict detector, as indexed by confidence. That is, this group showed conflict detection (i.e., decreased confidence) in both task formats. There were also two groups of inconsistent detectors: first, $23.2 \%$ of the biased reasoners were conflict detectors only in the problem-solving format; second, $20.8 \%$ detected conflict, only in the vignette format. The remaining $13.6 \%$ were consistent non-detectors.

Response time. For the response time index (Table S3), 22.4\% of the consistent biased reasoners $(n=125)$, were also consistent conflict detectors (i.e., increased response time) in both problem-solving and vignette tasks. Furthermore, $28.8 \%$ detected conflict only in the problem-solving tasks, and $23.2 \%$ detected conflict only in vignette tasks. The remaining $25.6 \%$ were consistent reversed detectors.

Confidence response time. Finally, $32.0 \%$ of the consistent biased reasoners $(n=125)$ showed conflict detection on the confidence response time index (i.e., increased confidence response time) in both task formats (Table S4). Also, 28.8\% showed conflict detection in the problem-solving tasks only, and $17.6 \%$ in the vignette tasks only. The other $21.6 \%$ did not detect conflict in any task format.

In sum, all three conflict detection indices indicated there was a group of consistent conflict detectors and a group of consistent non-detectors. The confidence index yielded the largest group of consistent detectors, followed by the confidence response time, and response time indices, respectively. There were also two groups of inconsistent detectors, which showed conflict detection in only one of the two task formats. All three indices indicated that more participants detected conflict in the problem-solving tasks (i.e., decision-making) than in the vignette tasks (i.e., decision-evaluation), although it were small differences. 
Table S5.2. Cross table showing the number of individuals who detected conflict on the base-rate tasks across both task formats, as indexed by confidence

\begin{tabular}{lcccc}
\hline & Detection & Reverse & Same & All correct \\
\cline { 2 - 5 } Problem-solving & 53 & 21 & 8 & 7 \\
Detection & 17 & 12 & 3 & 3 \\
Reverse & 9 & 1 & 1 & 0 \\
Same & 15 & 3 & 1 & 5 \\
All correct & & & & \\
\hline
\end{tabular}

Table S5.3. Cross table showing the number of individuals who detected conflict on the base-rate tasks across both task formats, as indexed by response time

\begin{tabular}{lccc}
\hline & Detection & $\begin{array}{c}\text { Vignette } \\
\text { Reverse }\end{array}$ & All correct \\
\cline { 2 - 4 } Problem-solving & & & \\
Detection & 28 & 36 & 5 \\
Reverse & 29 & 32 & 5 \\
All correct & 13 & 6 & 5 \\
\hline
\end{tabular}

Table S5.4. Cross table showing the number of individuals who detected conflict on the base-rate tasks across both task formats, as indexed by confidence response time

\begin{tabular}{lcccc}
\hline & Detection & Reverse & Same & All correct \\
\cline { 2 - 5 } Problem-solving & 40 & 36 & 0 & 7 \\
Detection & 22 & 27 & 0 & 3 \\
Reverse & 12 & 6 & 1 & 5 \\
All correct & & & & \\
\hline
\end{tabular}

\section{CONJUNCTION TASKS}

We conducted the same individual difference analyses for the conjunction tasks.

Number of detectors. As for the base-rate tasks, we tallied per conflict detection index how many of the biased reasoners showed the conflict detection effect. Results are shown in Table S1 (bottom two panels).

Confidence (\%). For the problem-solving tasks, we found that $72.0 \%$ of the biased reasoners showed conflict detection on the confidence index, with an average effect size of $-16.0 \%$ $(\mathrm{SD}=12.3)$. For the vignette tasks, we found that a somewhat smaller majority of the biased 
reasoners $(57.9 \%)$ showed conflict detection, yet with a similar effect size $(-16.2 \%$, SD $=11.9$ ). The percentage of conflict detectors on the problem-solving tasks was in line with Frey et al. (2018), who found a percentage of 79\%, yet the effect sizes on both task formats were smaller than the effect size $(-27.6 \%)$ in Frey et al. (2018).

Response time (s). For the response time index, we found that $55.9 \%$ of the biased participants showed conflict detection on the problem-solving tasks, with an average detection effect size of $9.9 \mathrm{~s}(\mathrm{SD}=14.1)$. Similarly, $54.5 \%$ of the biased participants showed conflict detection on the vignette tasks, with an average size of $9.2 \mathrm{~s}(\mathrm{SD}=7.3)$. These findings deviated from Frey et al., (2018) who found a larger group of conflict detectors $(71 \%)$, with a smaller effect size (3.0 s).

Confidence response time (s). For the problem-solving tasks, we found that $44.8 \%$ of the biased participants showed conflict detection on the confidence response time index, with an average effect size $0.6 \mathrm{~s}(\mathrm{SD}=0.7)$. Likewise, $42.1 \%$ of the biased participants showed conflict detection on the vignette tasks with an average effect size of $0.8 \mathrm{~s}(\mathrm{SD}=1.2)$. These findings were in line with Frey et al. (2018), who found that $48 \%$ of the biased participants showed conflict detection with a comparable effect size $1.1 \mathrm{~s}$.

In sum, also when taking individual differences into account, we found very similar conflict detection patterns for both problem-solving (i.e., decision-making) and vignette (i.e., decision-evaluation) tasks. Furthermore, apart from the response time index, the results on the current, more realistic versions of the conjunction tasks were very similar to what Frey et al. (2018) obtained on classical conjunction problems.

Accuracy correlations. We calculated the correlation between individuals' conflict detection effect size on the three conflict detection indices and their total accuracy on the conflict tasks (see Table S1, bottom two panels). The analyses yielded four significant correlations for the problem-solving tasks. Within the conflict detection group (and also in the whole biased group), a larger confidence effect size, $\mathrm{r}=-0.35, p<.001$, and a larger response time effect size, $\mathrm{r}=0.30, p=.008$, was related to higher accuracy on conflict problem-solving tasks. Hence, a lower confidence and longer response time on incorrectly solved conflict tasks (relative to correctly solved no-conflict tasks) was associated with higher accuracy on other conflict problem-solving tasks. For the vignette tasks, we obtained three significant correlations. Within the conflict detection group, a larger confidence effect size, $\mathrm{r}=-0.29$, $p=.008$, and confidence response time effect size, $\mathrm{r}=0.29, p=.022$, was related to higher accuracy on conflict vignette tasks. Within the reversed detection group, a larger reversed effect size was related to lower accuracy, $\mathrm{r}=0.28, p=.041$.

In sum, the correlations indicated that, for both for tasks formats, the size of the conflict detection effects could reflect individual differences in the quality of the detection process among biased reasoners. This was in contrast to Frey et al. (2018), who, for the classical conjunction problems, obtained only one significant correlation (on the response time index) in this direction. 
Conflict detection consistency. We used cross-tables to test to what extent reasoners were consistent conflict detectors across the two task formats (Tables S5-S7).

Confidence. Of all participants $(n=159), 82.4 \%(n=131)$ responded biased at least once to the conflict tasks in both task formats (Table S5). Of these consistent biased reasoners, $43.5 \%$ was also a consistent conflict detector in both task formats, as indicated by confidence ratings. There were again two groups of inconsistent detectors: $29.0 \%$ detected conflict in the problem-solving tasks only, and $16.8 \%$ detected conflict in the vignette tasks only. The remaining $10.7 \%$ were consistent non-detectors.

Response time. For the response time index (Table S6), 33.6\% of the consistent biased reasoners $(n=131)$, was also consistent conflict detector in both task formats. Furthermore, $22.9 \%$ only detected conflict on the problem-solving tasks, whereas $20.6 \%$ only detected conflict on the vignette tasks. The remaining $22.9 \%$ were consistent non-detectors.

Confidence response time. Finally, $17.6 \%$ of the consistent biased reasoners $(n=131)$. showed conflict detection on the confidence response time index in both task formats, $26.7 \%$ of the biased reasoners only showed conflict detection in the problem-solving format, and $22.1 \%$ only in the vignette format (Table S7). The final $33.6 \%$ did not detect conflict in any task format.

In sum, all three conflict detection indices showed there was one group of consistent conflict detectors and a group of consistent non-detectors. The confidence index yielded the largest group of consistent conflict detectors, followed by the response time index and confidence response time index, respectively. There was also a relatively large group of inconsistent detectors, meaning that they detected conflict either in the problem-solving format or in the vignette format. All three indices pointed out that slightly more participants detected conflict in the problem-solving format (i.e., decision-making) than in the vignette format (i.e., decision-evaluation).

Table S5.5. Cross table showing the number of individuals who detected conflict on the conjunction tasks across both task formats, as indexed by confidence

\begin{tabular}{lcccc}
\hline & Detection & Reverse & Same & All correct \\
\cline { 2 - 5 } Problem-solving & 57 & 35 & 3 & 8 \\
Detection & 16 & 11 & 1 & 4 \\
Reverse & 6 & 1 & 1 & 0 \\
Same & 5 & 7 & 2 & 2 \\
All correct & & & & \\
\hline
\end{tabular}


Table S5.6. Cross table showing the number of individuals who detected conflict on the conjunction tasks across both task formats, as indexed by response time

\begin{tabular}{lccc}
\hline & Detection & Vignette & Reverse \\
\cline { 2 - 4 } Problem-solving & & & All correct \\
Detection & 44 & 30 & 6 \\
Reverse & 27 & 30 & 6 \\
All correct & 8 & 6 & 2 \\
\hline
\end{tabular}

Table S5.7. Cross table showing the number of individuals who detected conflict on the conjunction tasks across both task formats, as indexed by confidence response time

\begin{tabular}{lccc}
\hline & Detection & Vignette & Reverse \\
\cline { 2 - 4 } Problem-solving & & & All correct \\
Detection & 23 & 35 & 4 \\
Reverse & 29 & 44 & 8 \\
All correct & 8 & 6 & 2 \\
\hline
\end{tabular}




\section{SUPPLEMENTARY MATERIALS - CHAPTER 6}

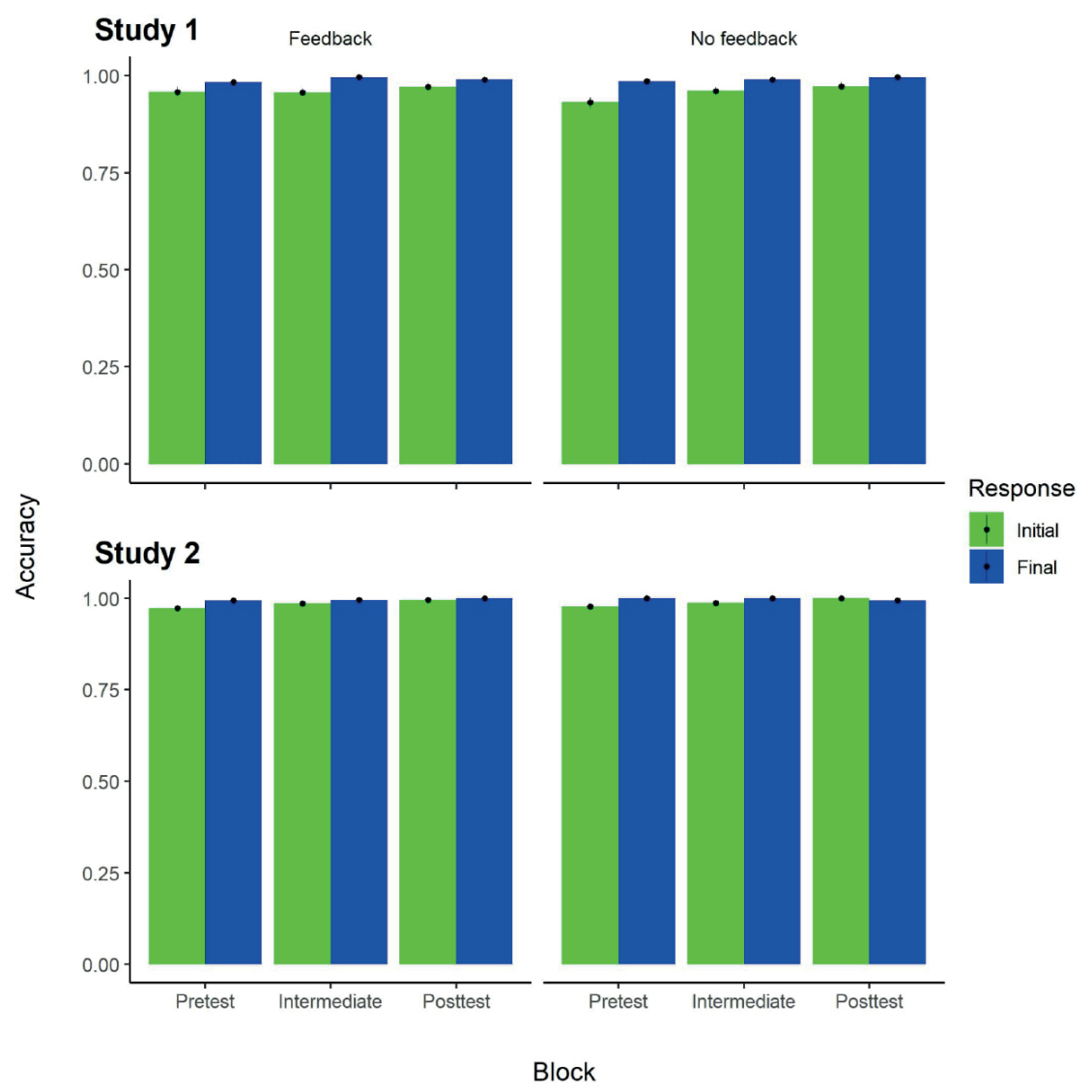

Figure S6.1. Average initial and final accuracy on no-conflict problems. Error bars are standard errors. 


\section{SUPPLEMENTARY MATERIALS}

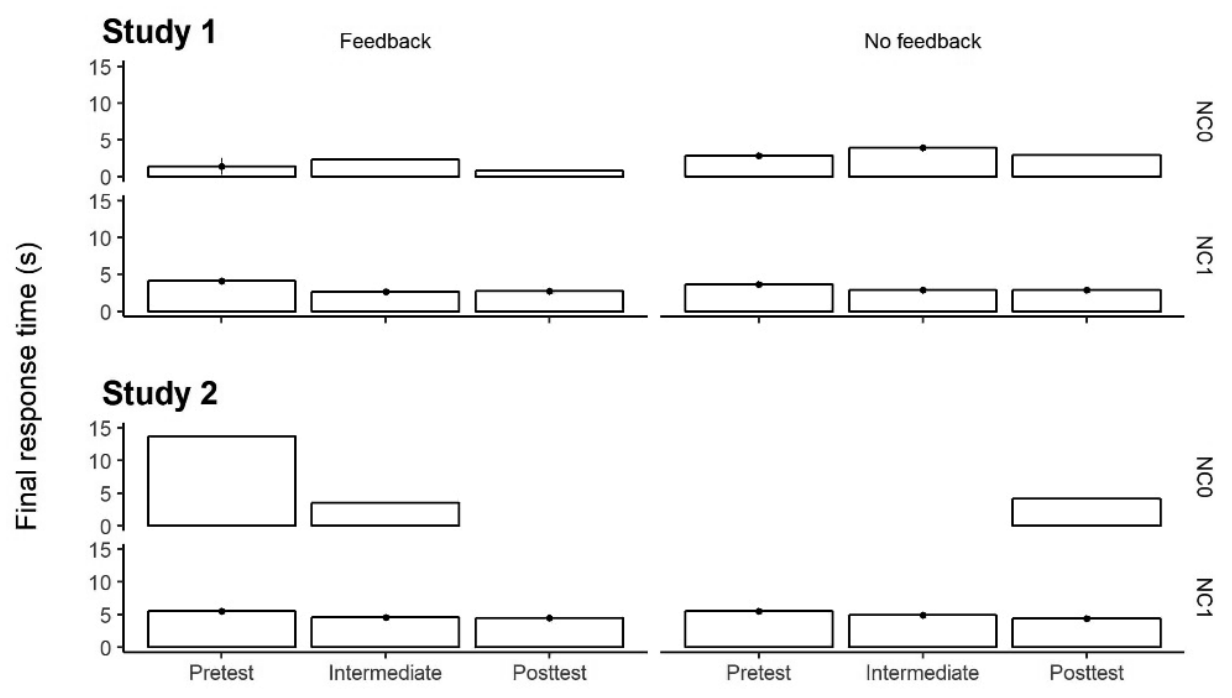

Block

Figure S6.2. Average, final response times on no-conflict problems. $\mathrm{NCO}=$ incorrect performance, $\mathrm{NC} 1$ $=$ correct performance. Error bars are standard errors. Note. Due to a technical failure, final response time of one no-conflict trial in the intermediate block and one no-conflict trial in posttest block is missing for all participants in Study 2. 


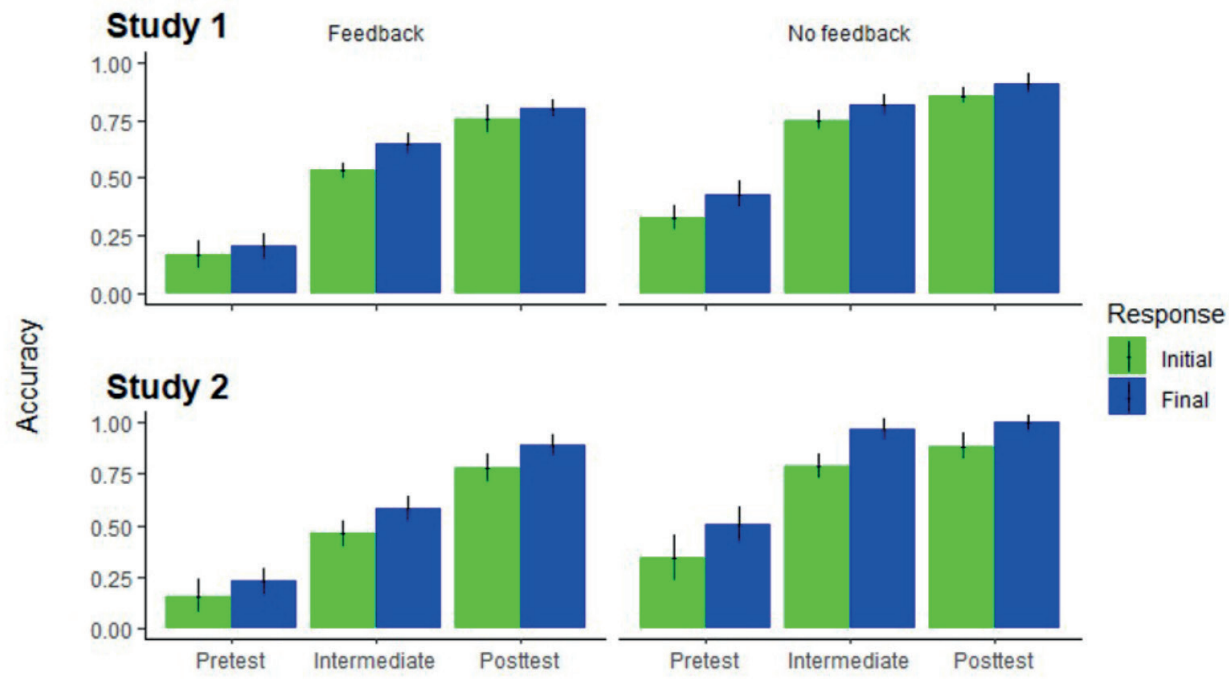

Block

Figure S6.3. Average initial and final accuracy on conflict problems for improved reasoners only. Error bars are standard errors. 
Table S6.1. Mixed-effects logistic regression model testing the effect of feedback

\begin{tabular}{lll}
\hline & Initial responses: & Final responses \\
\hline $\begin{array}{l}\text { Study } 1 \\
\text { Fixed effects }\end{array}$ & Coefficient (s.e.) & Coefficient (s.e.) \\
$\quad$ Intercept & $-9.19(1.37)^{\star \star}$ & $-15.39(2.03)^{\star *}$ \\
$\quad$ Block & $2.50(0.47)^{\star \star}$ & $5.54(1.49)^{\star \star}$ \\
$\quad$ Condition & $-0.76(1.38)$ & $1.56(2.13)$ \\
$\quad$ Block $\times$ Condition & $1.29(0.72)$ & $-1.21(1.65)$ \\
Random effects & & \\
$\quad$ Subject & $74.81(8.65)$ & $381.1(19.52)$ \\
Study 2 & Coefficient (s.e.) & Coefficient (s.e.) \\
Fixed effects & & \\
$\quad$ Intercept & $-15.88(2.36)^{\star *}$ & $-13.44(1.79)^{\star *}$ \\
$\quad$ Block & $6.17(1.68)^{\star *}$ & $3.10(0.86)^{\star *}$ \\
$\quad$ Condition & $-4.52(2.87)$ & $-6.44(3.77)$ \\
$\quad$ Block $\times$ Condition & $4.44(2.50)$ & $6.33(3.42)$ \\
Random effects & & \\
$\quad$ Subject & $280.6(16.75)$ & $272.7(16.51)$ \\
\hline
\end{tabular}

Note. Condition coded $0=$ no-feedback condition, $1=$ feedback condition. Block coded $0=$ pretest, 1 $=$ posttest. ${ }^{*} p<.05,{ }^{\star *} p<.001$. 
Table S6.2. ANOVAs with sex as covariate and as moderator

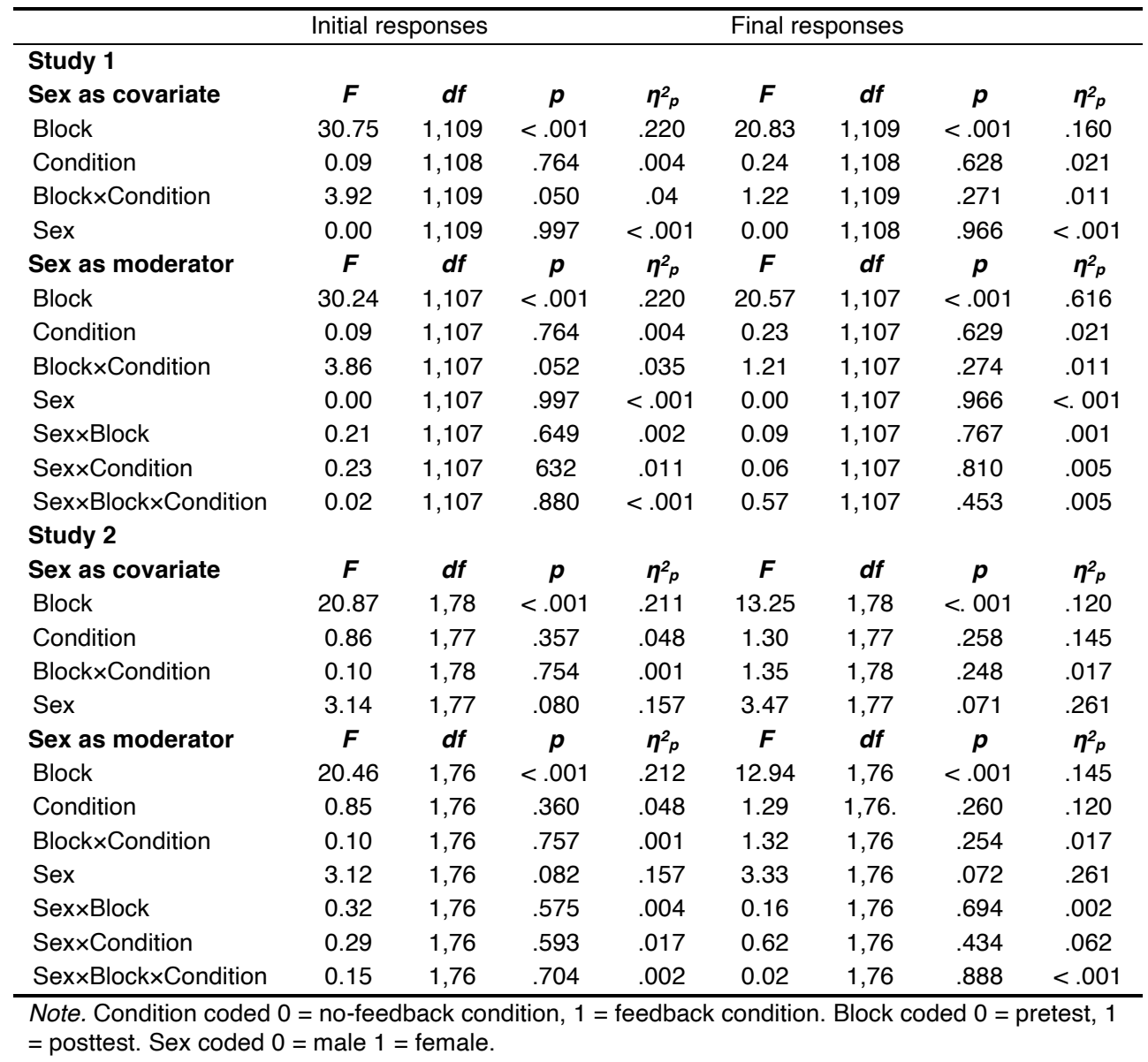


Table S6.3. Mixed-effects logistic regression model testing the effect of feedback with sex as covariate

\begin{tabular}{|c|c|c|}
\hline & Initial responses: & Final responses \\
\hline Study 1 & Coefficient (s.e.) & Coefficient (s.e.) \\
\hline \multicolumn{3}{|l|}{ Fixed effects } \\
\hline Intercept & $-9.23(1.57)^{\star \star}$ & $-15.38(2.23)^{\star \star}$ \\
\hline Block & $2.49(0.47)^{\star \star}$ & $5.54(1.49)^{* *}$ \\
\hline Condition & $-0.69(1.41)$ & $1.57(2.13)$ \\
\hline Block $\times$ Condition & $1.29(0.72)$ & $-1.21(1.65)$ \\
\hline Sex & $0.17(1.24)$ & $-0.01(1.46)$ \\
\hline \multicolumn{3}{|l|}{ Random effects } \\
\hline Subject & $73.3(8.56)$ & $380.5(19.51)$ \\
\hline Study 2 & Coefficient (s.e.) & Coefficient (s.e.) \\
\hline \multicolumn{3}{|l|}{ Fixed effects } \\
\hline Intercept & $-15.19(2.57)^{\star \star}$ & $-12.75(2.09)^{\star \star}$ \\
\hline Block & $6.15(1.67)^{\star \star}$ & $3.09(0.86)^{\star \star}$ \\
\hline Condition & $-4.47(2.87)$ & $-6.32(3.78)$ \\
\hline Block $\times$ Condition & $4.38(2.49)$ & $6.22(3.42)$ \\
\hline Sex & $-1.00(1.71)$ & $-1.05(1.86)$ \\
\hline \multicolumn{3}{|l|}{ Random effects } \\
\hline Subject & $272.7(16.51)$ & $417.1(20.42)$ \\
\hline
\end{tabular}

Note. Condition coded $0=$ no-feedback condition, $1=$ feedback condition. Block coded $0=$ pretest, 1 $=$ posttest. Sex coded $0=$ male $1=$ female. ${ }^{*} p<.05,{ }^{* \star} p<.001$. 
Table S6.4. Mixed-effects logistic regression model testing the effect of feedback with sex as moderator

\begin{tabular}{|c|c|c|}
\hline & Initial responses: & Final responses \\
\hline \multicolumn{3}{|l|}{ Study 1} \\
\hline Fixed effects & Coefficient (s.e.) & Coefficient (s.e.) \\
\hline Intercept & $-8.91(1.86)^{\star *}$ & $-14.65(2.57)^{\star *}$ \\
\hline Block & $2.34(0.82)^{\star \star}$ & $4.37(1.79)^{\star}$ \\
\hline Condition & $-0.93(2.13)$ & $-7.05(3.65)$ \\
\hline Block $\times$ Condition & $1.08(1.15)$ & $7.48(2.96)^{\star}$ \\
\hline Sex & $-0.28(1.77)$ & $-3.47(3.96)$ \\
\hline Sex $\times$ Block & $0.21(0.97)$ & $3.33(3.47)$ \\
\hline Sex $\times$ Condition & $0.41(2.87)$ & $12.20(5.35)^{\star}$ \\
\hline Sex $\times$ Block $\times$ Condition & $0.41(1.49)$ & $-12.22(4.57)^{\star}$ \\
\hline \multicolumn{3}{|l|}{ Random effects } \\
\hline \multicolumn{3}{|l|}{ Subject } \\
\hline \multicolumn{3}{|l|}{ Study 2} \\
\hline Fixed effects & Coefficient (s.e.) & Coefficient (s.e.) \\
\hline Intercept & $-12.96(2.40)^{\star \star}$ & $-12.06(2.28)^{\star \star}$ \\
\hline Block & $3.62(1.23)^{\star \star}$ & $2.46(0.97)^{\star}$ \\
\hline Condition & $-6.21(4.89)$ & $-8.05(4.97)$ \\
\hline Block $\times$ Condition & $5.76(4.32)$ & $7.69(4.36)$ \\
\hline Sex & $-8.43(3.67)^{\star}$ & $-3.43(3.36)$ \\
\hline Sex $\times$ Block & $7.56(2.97)^{\star}$ & $2.21(2.24)$ \\
\hline Sex $\times$ Condition & $5.76(6.32)$ & $3.99(6.73)$ \\
\hline Sex $\times$ Block $\times$ Condition & $-5.27(5.59)$ & $-3.53(5.85)$ \\
\hline \multicolumn{3}{|l|}{ Random effects } \\
\hline Subject & $321.2(17.92)$ & $429.7(20.73)$ \\
\hline
\end{tabular}

Note. Condition coded $0=$ no-feedback condition, $1=$ feedback condition. Block coded $0=$ pretest, 1 $=$ posttest. Sex coded $0=$ male $1=$ female. ${ }^{*} p<.05,{ }^{* \star} p<.001$. a We broke down the final response's significant interaction; results indicated that the feedback was effective for males $(p<.001)$ not for females $(p=.142)$. 
Table S6.5. Frequency of different types of justifications for the final bat-and-ball problem in Study 2

\begin{tabular}{|c|c|c|c|c|}
\hline \multirow[b]{2}{*}{ Justification } & \multicolumn{2}{|c|}{ Feedback condition } & \multicolumn{2}{|c|}{ No-feedback condition } \\
\hline & $\begin{array}{l}\text { Correct } \\
(n=13)\end{array}$ & $\begin{array}{l}\text { Incorrect } \\
(n=24)\end{array}$ & $\begin{array}{l}\text { Correct } \\
(n=14)\end{array}$ & $\begin{array}{l}\text { Incorrect } \\
(n=25)\end{array}$ \\
\hline Math - correct & 11 & - & 14 & - \\
\hline Math - incorrect & - & 16 & - & 18 \\
\hline Math - unspecified & - & 1 & - & 2 \\
\hline Guess & 1 & 2 & - & - \\
\hline Intuition & 1 & 4 & - & 4 \\
\hline Other - correct & - & - & - & 1 \\
\hline Other - incorrect & - & 1 & - & - \\
\hline Other - unspecified & - & - & - & - \\
\hline
\end{tabular}

Table S6.6. Average proportion of correct responses on the two transfer problems for improved versus unimproved reasoners

\begin{tabular}{clll}
\hline & $n$ & $\mathrm{M}$ & $\mathrm{SE}$ \\
\hline Feedback condition & & & \\
Improved reasoners & 9 & .56 & .13 \\
Unimproved reasoners & 31 & .44 & .08 \\
no ceiled pretest & 27 & .38 & .08 \\
ceiled pretest & 4 & .88 & .13 \\
No-feedback condition & & & \\
Improved reasoners & 7 & .64 & .14 \\
Unimproved reasoners & 33 & .50 & .08 \\
no ceiled pretest & 25 & .36 & .08 \\
ceiled pretest & 8 & .94 & .07 \\
\hline
\end{tabular}

Note. For the unimproved reasoners, we additionally distinguished between those who had no ceiled pretest performance (i.e., could still improve but simply did not) and those who had a ceiled pretest performance (i.e., were unable to improve). 
SUPPLEMENTARY MATERIALS 


\section{REFERENCES}


Abrami, P. C., Bernard, R. M., Borokhovski, E., Waddington, D. I., Wade, C. A., \& Persson, T. (2015). Strategies for teaching students to think critically: A meta-analysis. Review of Educational Research, 85, 275-314. doi:10.3102/0034654314551063

Abrami, P. C., Bernard, R. M., Borokhovski, E., Wade, A., Surkes, M. A., Tamim, R., \& Zhang, D. (2008). Instructional interventions affecting critical thinking skills and dispositions: A stage 1 meta-analysis. Review of Educational Research, 78, 1102-1134. doi:10.3102/0034654308326084

Aczel, B., Bago, B., Szollosi, A., Foldes, A., \& Lukacs, B. (2015). Measuring individual differences in decision biases: Methodological considerations. Frontiers in Psychology, 6. doi:10.3389/fpsyg.2015.01770

Alexander, P. A. (2013). Calibration: What is it and why it matters? An introduction to the special issue on calibrating calibration. Learning and Instruction, 24, 1-3. doi:10.1016/j.learninstruc.2012.10.003

Anwyl-Irvine, A., Massonnié, J., Flitton, A., Kirkham, N., \& Evershed, J. (2018). Gorilla in our Midst: An online behavioral experiment builder [Preprint]. Neuroscience. doi:10.1101/438242

Arum, R., Cho, E., Kim, J., \& Roksa, J. (2012). Documenting uncertain times: Post-graduate transitions of the Academically Adrift cohort. Social Science Research Council.

Arum, R., \& Roksa, J. (2011). Limited learning on college campuses. Society, 48, 203-207. doi:10.1007/s12115-011-9417-8

Athanasiou, K., Katakos, E., \& Papadopoulou, P. (2012). Conceptual ecology of evolution acceptance among Greek education students: The contribution of knowledge increase. Journal of Biological Education, 46, 234-241. doi:10.1080/00219266.20 12.716780

Athanasiou, K., \& Papadopoulou, P. (2012). Conceptual ecology of the evolution acceptance among Greek education students: Knowledge, religious practices and social influences. International Journal of Science Education, 34, 903-924. doi:10 $.1080 / 09500693.2011 .586072$

Athanasiou, K., \& Papadopoulou, P. (2015). Evolution theory teaching and learning: What conclusions can we get from comparisons of teachers' and students' conceptual ecologies in greece and Turkey? EURASIA Journal of Mathematics, Science and Technology Education, 11, 841-853. doi:10.12973/eurasia.2015.1443a

Bago, B., \& De Neys, W. (2017). Fast logic?: Examining the time course assumption of dual process theory. Cognition, 158, 90-109. doi:10.1016/j.cognition.2016.10.014

Bago, B., \& De Neys, W. (2019). The Smart System 1: Evidence for the intuitive nature of correct responding on the bat-and-ball problem. Thinking \& Reasoning, 25, 257-299. doi:10.1080/13546783.2018.1507949

Bago, B., Raoelison, M., \& De Neys, W. (2019). Second-guess: Testing the specificity of error detection in the bat-and-ball problem. Acta Psychologica, 193, 214-228. doi:10.1016/j.actpsy.2019.01.008

Ball, L. J. (2013). Microgenetic evidence for the beneficial effects of feedback and practice on belief bias. Journal of Cognitive Psychology, 25, 183-191. doi:10.1080/204459 11.2013.765856

Ball, L. J., Hoyle, A. M., \& Towse, A. S. (2010). The facilitatory effect of negative feedback on the emergence of analogical reasoning abilities. British Journal of Developmental Psychology, 28, 583-602. doi:10.1348/026151009X461744 


\section{REFERENCES}

Bandura, A. (1982). Self-efficacy mechanism in human agency. American Psychologist, 37, 122-147. doi:10.1037/0003-066X.37.2.122

Barnet, S., Bedau, H. A., \& O’Hara, J. (2017). Critical thinking, reading, and writing: A brief guide to argument.

Baron, J. (1991). Beliefs about thinking. In J. F. Voss, D. N. Perkins, \& J. W. Segal (Eds.), Informal reasoning and education (pp. 169-186). Hillsdale. https://www.sas. upenn.edu/ baron/papers/voss.pdf

Baron, J. (2008). Thinking and deciding (4. ed). Cambridge University Press.

Baron, J., Scott, S., Fincher, K., \& Emlen Metz, S. (2015). Why does the Cognitive Reflection Test (sometimes) predict utilitarian moral judgment (and other things)? Journal of Applied Research in Memory and Cognition, 4, 265-284. doi:10.1016/j. jarmac.2014.09.003

Baruch, Y., \& Holtom, B. C. (2008). Survey response rate levels and trends in organizational research. Human Relations, 61, 1139-1160. doi:10.1177/0018726708094863

Bates, D., Mächler, M., Bolker, B., \& Walker, S. (2015). Fitting linear mixed-effects models using lme4. Journal of Statistical Software, 67, 1-48. doi:10.18637/jss.v067.i01

Bautista, N., Misco, T., \& Quaye, S. J. (2018). Early childhood open-mindedness: An investigation into preservice teachers' capacity to address controversial issues. Journal of Teacher Education, 69, 154-168. doi:10.1177/0022487117702575

Beatty, E. L., \& Thompson, V. A. (2012). Effects of perspective and belief on analytic reasoning in a scientific reasoning task. Thinking \& Reasoning, 18, 441-460. doi:1 $0.1080 / 13546783.2012 .687892$

Bernard, R. M., Zhang, D., Abrami, P. C., Sicoly, F., Borokhovski, E., \& Surkes, M. A. (2008). Exploring the structure of the Watson-Glaser Critical Thinking Appraisal: One scale or many subscales? Thinking Skills and Creativity, 3, 15-22. doi:10.1016/j.tsc.2007.11.001

Bondy, K. N., Koenigseder, L. A., Ishee, J. H., \& Williams, B. G. (2001). Psychometric properties of the California Critical Thinking Tests. Journal of Nursing Measurement, 9, 309-328. doi:10.1891/1061-3749.9.3.309

Bonner, C., \& Newell, B. R. (2010). In conflict with ourselves? An investigation of heuristic and analytic processes in decision making. Memory \& Cognition, 38, 186-196. doi:10.3758/MC.38.2.186

Bosch-Domènech, A., Brañas-Garza, P., \& Espín, A. M. (2014). Can exposure to prenatal sex hormones (2D:4D) predict cognitive reflection? Psychoneuroendocrinology, 43, 1-10. doi:10.1016/j.psyneuen.2014.01.023

Brañas-Garza, P., Kujal, P., \& Lenkei, B. (2019). Cognitive reflection test: Whom, how, when. Journal of Behavioral and Experimental Economics, 82, 101455. doi:10.1016/j.socec.2019.101455

Brown, T. A. (2006). Confirmatory factor analysis for applied research. Guilford Press.

Bruine de Bruin, W., Parker, A. M., \& Fischhoff, B. (2007). Individual differences in adult decision-making competence. Journal of Personality and Social Psychology, 92, 
938-956. doi:10.1037/0022-3514.92.5.938

Cacioppo, J. T., \& Petty, R. E. (1982). The need for cognition. Journal of Personality and Social Psychology, 42, 116-131. doi:10.1037/0022-3514.42.1.116

Cacioppo, J. T., Petty, R. E., \& Feng Kao, C. (1984). The efficient assessment of Need for Cognition. Journal of Personality Assessment, 48, 306-307. doi:10.1207/ s15327752jpa4803_13

Campitelli, G., \& Gerrans, P. (2014). Does the cognitive reflection test measure cognitive reflection? A mathematical modeling approach. Memory \& Cognition, 42, 434-447. doi:10.3758/s13421-013-0367-9

Charter, R. A. (2003). A Breakdown of Reliability Coefficients by Test Type and Reliability Method, and the Clinical Implications of Low Reliability. The Journal of General Psychology, 130, 290-304. doi:10.1080/00221300309601160

Chiesi, F., Morsanyi, K., Donati, M. A., \& Primi, C. (2018). Applying item response theory to develop a shortened version of the Need for Cognition scale. Advances in Cognitive Psychology, 14, 75-86. doi:10.5709/acp-0240-z

Choy, S. C., \& Cheah, P. K. (2009). Teacher perceptions of critical thinking among students and its influence on higher education. International Journal of Teaching and Learning in Higher Education, 20, 198-206.

Chun, M. M., \& Wolfe, J. M. (1996). Just say No: How are visual searches terminated when there is no target present? Cognitive Psychology, 30, 39-78. doi:10.1006/ cogp. 1996.0002

Cicchetti, D. V. (1994). Guidelines, criteria, and rules of thumb for evaluating normed and standardized assessment instruments in psychology. Psychological Assessment, 6, 284-290. doi:10.1037/1040-3590.6.4.284

Cohen, A. R., Stotland, E., \& Wolfe, D. M. (1955). An experimental investigation of need for cognition. The Journal of Abnormal and Social Psychology, 51(2), 291-294. doi:10.1037/h0042761

Cohen, J. (1988). Statistical power analysis for the behavioral sciences (2. ed., reprint). Psychology Press.

Dawson, E., Gilovich, T., \& Regan, D. T. (2002). Motivated reasoning and performance on the Wason selection task. Personality and Social Psychology Bulletin, 28, 1379-1387. doi:10.1177/014616702236869

De Neys, W. (2012). Bias and conflict: A case for logical intuitions. Perspectives on Psychological Science, 7, 28-38. doi:10.1177/1745691611429354

De Neys, W. (2014). Conflict detection, dual processes, and logical intuitions: Some clarifications. Thinking \& Reasoning, 20, 169-187. doi:10.1080/13546783.2013.8 54725

De Neys, W. (Ed.). (2017). Dual process theory 2.0. Routledge, Taylor \& Francis Group.

De Neys, W., \& Bonnefon, J.-F. (2013). The 'whys' and 'whens' of individual differences in thinking biases. Trends in Cognitive Sciences, 17, 172-178. doi:10.1016/j. tics.2013.02.001

De Neys, W., Cromheeke, S., \& Osman, M. (2011). Correction: Biased but in doubt: 
Conflict and decision confidence. PLOS ONE, 6. doi:10.1371/annotation/1ebd8050-5513-426f-8399-201773755683

De Neys, W., \& Feremans, V. (2013). Development of heuristic bias detection in elementary school. Developmental Psychology, 49, 258-269. doi:10.1037/a0028320

De Neys, W., \& Glumicic, T. (2008). Conflict monitoring in dual process theories of thinking. Cognition, 106, 1248-1299. doi:10.1016/j.cognition.2007.06.002

De Neys, W., \& Pennycook, G. (2019). Logic, fast and slow: Advances in dual-process theorizing. Current Directions in Psychological Science, 28, 503-509. doi:10.1177/0963721419855658

De Neys, W., Rossi, S., \& Houdé, O. (2013). Bats, balls, and substitution sensitivity: Cognitive misers are no happy fools. Psychonomic Bulletin \& Review, 20, 269-273. doi:10.3758/s13423-013-0384-5

Deniz, H. (2011). Examination of changes in prospective elementary teachers' epistemological beliefs in science and exploration of factors meditating that change. Journal of Science Education and Technology, 20, 750-760. doi:10.1007/ s10956-010-9268-X

Deniz, H., Donnelly, L. A., \& Yilmaz, I. (2008). Exploring the factors related to acceptance of evolutionary theory among Turkish preservice biology teachers: Toward a more informative conceptual ecology for biological evolution. Journal of Research in Science Teaching, 45, 420-443. doi:10.1002/tea.20223

Donnelly, N., Cave, K., Greenway, R., Hadwin, J. A., Stevenson, J., \& Sonuga-Barke, E. (2007). Visual search in children and adults: Top-down and bottom-up mechanisms. Quarterly Journal of Experimental Psychology, 60, 120-136. doi:10.1080/17470210600625362

Dumitru, D. (2018, June 20). University teachers' thoughts about how critical thinking is a part of their classes. International Conference on Technology and Innovation in Learning, Teaching and Education (TECH-EDU 2018), Thessaloniki, Greece. doi:10.1007/978-3-030-20954-4_15

Eberhardt, J. L., Davies, P. G., Purdie-Vaughns, V. J., \& Johnson, S. L. (2006). Looking deathworthy: Perceived stereotypicality of black defendants predicts capital-sentencing outcomes. Psychological Science, 17, 383-386. doi:10.1111/ j.1467-9280.2006.01716.x

Eccles, J. S., \& Wigfield, A. (2002). Motivational beliefs, values, and goals. Annual Review of Psychology, 53, 109-132. doi:10.1146/annurev.psych.53.100901.135153

Elen, J., Jiang, L., Huyghe, S., Evers, M., Verburgh, A., ..., \& Palaigeorgiou, G. (2019). Promoting Critical Thinking in European Higher Education Institutions: Towards an educational protocol. Vila Real: UTAD.

Elik, N., Wiener, J., \& Corkum, P. (2010). Pre-service teachers' open-minded thinking dispositions, readiness to learn, and attitudes about learning and behavioural difficulties in students. European Journal of Teacher Education, 33, 127-146. doi:10.1080/02619760903524658

Ennis, R. H. (1987). A taxonomy of critical thinking dispositions and abilities. In J. Baron 
\& R. J. Sternberg (Eds.), Teaching thinking skills: Theory and practice (pp. 9-26). Freeman.

Ennis, R. H., Millman, J., \& Tomko, T. N. (1985). Cornell critical thinking tests (3erd ed.). Midwest Publications.

Epstein, S., Pacini, R., Denes-Raj, V., \& Heier, H. (1996). Individual differences in intuitive-experiential and analytical-rational thinking styles. Journal of Personality and Social Psychology, 71(2), 390-405. doi:10.1037/00223514.71.2.390

Evans, J. St. B. T. (2002). Logic and human reasoning: An assessment of the deduction paradigm. Psychological Bulletin, 128, 978-996. doi:10.1037//00332909.128.6.978

Evans, J. St. B. T. (2008). Dual-processing accounts of reasoning, judgment, and social cognition. Annual Review of Psychology, 59, 255-278. doi:10.1146/annurev. psych.59.103006.093629

Evans, J. St. B. T., Handley, S. J., \& Harper, C. N. J. (2001). Necessity, possibility and belief: A study of syllogistic reasoning. The Quarterly Journal of Experimental Psychology Section A, 54, 935-958. doi:10.1080/02724980042000417

Evans, J. St. B. T., \& Over, D. E. (1996). Rationality and reasoning. Psychology.

Evans, J. St. B. T., \& Stanovich, K. E. (2013). Dual-Process theories of higher cognition: Advancing the debate. Perspectives on Psychological Science, 8, 223-241. doi:10.1177/1745691612460685

Facione, P. A. (1990a). Critical thinking: A statement of expert consensus for purposes of educational assessment and instruction. Millbrae, CA: The California Academic Press.

Facione, P. A. (1990b). The California Critical Thinking Skills Test. California Academic Press.

Faul, F., Erdfelder, E., Lang, A.-G., \& Buchner, A. (2007). G*Power 3: A flexible statistical power analysis program for the social, behavioral, and biomedical sciences. Behavior Research Methods, 39(2), 175-191. doi:10.3758/BF03193146

Ferreira, M. B., Mata, A., Donkin, C., Sherman, S. J., \& Ihmels, M. (2016). Analytic and heuristic processes in the detection and resolution of conflict. Memory \& Cognition, 44, 1050-1063. doi:10.3758/s13421-016-0618-7

Fiorella, L., \& Mayer, R. E. (2016). Eight ways to promote generative learning. Educational Psychology Review, 28, 717-741. doi:10.1007/s10648-015-9348-9

Flora, D. B., LaBrish, C., \& Chalmers, R. P. (2012). Old and new ideas for data screening and assumption testing for exploratory and confirmatory factor analysis. Frontiers in Psychology. doi:10.3389/fpsyg.2012.00055

Fong, G. T., Krantz, D. H., \& Nisbett, R. E. (1986). The effects of statistical training on thinking about everyday problems. Cognitive Psychology, 18, 253-292. doi:10.1016/0010-0285(86)90001-0

Frederick, S. (2005). Cognitive reflection and decision making. Journal of Economic Perspectives, 19, 25-42. doi:10.1257/089533005775196732 


\section{REFERENCES}

Frey, D., \& De Neys, W. (2017). Is conflict detection in reasoning domain general ? Proceedings of the Annual Meeting of the Cognitive Science Society, 39, 391-396. https://pdfs.semanticscholar.org/aaec/4079baaeba9ba75c6cf816874e5cc2b9a201. pdf

Frey, D., Johnson, E. D., \& De Neys, W. (2018). Individual differences in conflict detection during reasoning. Quarterly Journal of Experimental Psychology, 1-52. doi:10.10 $80 / 17470218.2017 .1313283$

Galesic, M., \& Bosnjak, M. (2009). Effects of questionnaire length on participation and indicators of response quality in a web survey. Public Opinion Quarterly, 73, 349-360. doi:10.1093/poq/nfp031

Gangemi, A., Bourgeois-Gironde, S., \& Mancini, F. (2015). Feelings of error in reasoning - In search of a phenomenon. Thinking \& Reasoning, 21, 383-396. doi: $10.1080 / 13546783.2014 .980755$

Gerber, S., \& Scott, L. (2011). Gamers and gaming context: Relationships to critical thinking: Gamers and critical thinking. British Journal of Educational Technology, 42, 842-849. doi:10.1111/j.1467-8535.2010.01106.x

Gigerenzer, G., Hell, W., \& Blank, H. (1988). Presentation and content: The use of base rates as a continuous variable. Journal of Experimental Psychology: Human Perception and Performance, 14, 513-525. doi:10.1037/0096-1523.14.3.513

Gigerenzer, G., \& Hug, K. (1992). Domain-specific reasoning: Social contracts, cheating, and perspective change. Cognition, 43, 127-171. doi:10.1016/00100277(92)90060-U

Halpern, D. F. (1998). Teaching Critical Thinking for Transfer across Domains. American Psychologist, 53, 449-455. doi:10.1037//0003-066X.53.4.449

Haran, U., Ritov, I., \& Mellers, B. A. (2013). The role of actively open-minded thinking in information acquisition, accuracy, and calibration. Judgment and Decision Making, 8, 188-201.

Hattie, J. (2003, October). Teachers make a difference: What is the research evidence? Building Teacher Quality: What does the research tell us ACER Research Conference, Melbourne, Australia. http://research.acer.edu.au/research_ conference_2003/4/

Hattie, J. (2013). Calibration and confidence: Where to next? Learning and Instruction, 24, 62-66. doi:10.1016/j.learninstruc.2012.05.009

Hawkins, J., Pea, R. D., Glick, J., \& Scribner, S. (1984). "Merds that laugh don't like mushrooms": Evidence for deductive reasoning by preschoolers. Developmental Psychology, 20, 584-594. doi:10.1037/0012-1649.20.4.584

Hays, M. J., Kornell, N., \& Bjork, R. A. (2010). The costs and benefits of providing feedback during learning. Psychonomic Bulletin \& Review, 17, 797-801. doi:10.3758/PBR.17.6.797

Heijltjes, A., Van Gog, T., Leppink, J., \& Paas, F. (2014). Improving critical thinking: Effects of dispositions and instructions on economics students' reasoning skills. Learning and Instruction, 29, 31-42. doi:10.1016/j.learninstruc.2013.07.003 
Heijltjes, A., Van Gog, T., Leppink, J., \& Paas, F. (2015). Unraveling the effects of critical thinking instructions, practice, and self-explanation on students' reasoning performance. Instructional Science, 43, 487-506. doi:10.1007/s11251-015-9347-8

Heijltjes, A., Van Gog, T., \& Paas, F. (2014). Improving Students' Critical Thinking: Empirical Support for Explicit Instructions Combined with Practice: Critical thinking instructions. Applied Cognitive Psychology, 28, 518-530. doi:10.1002/ acp.3025

Hoffman, B., \& Schraw, G. (2010). Conceptions of efficiency: Applications in learning and problem solving. Educational Psychologist, 45, 1-14. doi:10.1080/00461520903213618

Hoover, J. D., \& Healy, A. F. (2017). Algebraic reasoning and bat-and-ball problem variants: Solving isomorphic algebra first facilitates problem solving later. Psychonomic Bulletin \& Review, 24, 1922-1928. doi:10.3758/s13423-017-1241-8

Hoover, J. D., \& Healy, A. F. (2019). The bat-and-ball problem: Stronger evidence in support of a conscious error process. Decision, 6, 369-380. doi:10.1037/ $\operatorname{dec} 0000107$

Hox, J. J. (2010). Multilevel analysis: Techniques and applications (2. ed). Routledge, Taylor \& Francis.

Hu, L., \& Bentler, P. M. (1999). Cutoff criteria for fit indexes in covariance structure analysis: Conventional criteria versus new alternatives. Structural Equation Modeling: A Multidisciplinary Journal, 6, 1-55. doi:10.1080/10705519909540118

Huber, C. R., \& Kuncel, N. R. (2016). Does college teach critical thinking? A meta-analysis. Review of Educational Research, 86, 431-468. doi: $10.3102 / 0034654315605917$

Jacobs, S. S. (1999). The equivalence of forms A and B of the California Critical Thinking Skills Test. Measurement and Evaluation in Counseling and Development, 31, 211-222.

Jacowitz, K. E., \& Kahneman, D. (1995). Measures of anchoring in estimation tasks. Personality and Social Psychology Bulletin, 21, 1161-1166. doi:10.1177/01461672952111004

Jamieson, S. (2004). Likert scales: How to (ab)use them. Medical Education, 38, 1217-1218. doi:10.1111/j.1365-2929.2004.02012.x

Janssen, E. M., Mainhard, T., Buisman, R. S. M., Verkoeijen, P. P. J. L., Heijltjes, A. E. G., Van Peppen, L. M., \& Van Gog, T. (2019). Training higher education teachers' critical thinking and attitudes towards teaching it. Contemporary Educational Psychology, 58, 310-322. doi:10.1016/j.cedpsych.2019.03.007

Janssen, E. M., Meulendijks, W., Mainhard, T., Verkoeijen, P. P. J. L., Heijltjes, A. E. G., Van Peppen, L. M., \& Van Gog, T. (2019). Identifying characteristics associated with higher education teachers' Cognitive Reflection Test performance and their attitudes towards teaching critical thinking. Teaching and Teacher Education, 84, 139-149. doi:10.1016/j.tate.2019.05.008

Johnson, E. D., Tubau, E., \& De Neys, W. (2016). The doubting System 1: Evidence for 


\section{REFERENCES}

automatic substitution sensitivity. Acta Psychologica, 164, 56-64. doi:10.1016/j. actpsy.2015.12.008

Jones, A. (2007). Multiplicities or manna from heaven? Critical thinking and the disciplinary context. Australian Journal of Education, 51, 84-103. doi:10.1177/000494410705100107

Jones, A., \& Moreland, J. (2003). Considering pedagogical content knowledge in the context of research on teaching: An example from technology. Waikato Journal of Education, 9. doi:10.15663/wje.v9i0.387

Jurkovič, M. (2016). Effect of short-term mindfulness induction on myside bias and miserly processing: A preliminary study. Studia Psychologica, 58, 231-237. doi:10.21909/ sp.2016.03.719

Kahneman, D. (2011). Thinking, fast and slow. Lane.

Kahneman, D., \& Frederick, S. (2005). A model of heuristic judgment. In K. J. Holyoak \& R. G. Morrison (Eds.), The Cambridge Handbook of Thinking and Reasoning (pp. 267-293). Cambridge University Press.

Kahneman, D., \& Tversky, A. (1973). On the psychology of prediction. Psychological Review, 80, 237-251. doi:10.1037/h0034747

Kanyongo, G. Y., Brook, G. P., Kyei-Blankson, L., \& Gocmen, G. (2007). Reliability and statistical power: How measurement fallibility affects power and required sample sizes for several parametric and nonparametric statistics. Journal of Modern Applied Statistical Methods, 6, 81-90. doi:10.22237/jmasm/1177992480

Kenyon, T., \& Beaulac. (2014). Critical thinking education and debiasing. Informal Logic, 34, 341. doi:10.22329/il.v34i4.4203

Klaczynski, P. A. (2014). Heuristics and biases: Interactions among numeracy, ability, and reflectiveness predict normative responding. Frontiers in Psychology, 5. doi:10.3389/fpsyg.2014.00665

Klassen, R. M., \& Tze, V. M. C. (2014). Teachers' self-efficacy, personality, and teaching effectiveness: A meta-analysis. Educational Research Review, 59-76. doi:10.1016/j.edurev.2014.06.001

Krumrei-Mancuso, E. J., \& Rouse, S. V. (2016). The development and validation of the Comprehensive Intellectual Humility Scale. Journal of Personality Assessment, 98, 209-221. doi:10.1080/00223891.2015.1068174

Kruyen, P. M., Emons, W. H. M., \& Sijtsma, K. (2012). Test length and decision quality in personnel selection: When is short too short? International Journal of Testing, 12, 321-344. doi:10.1080/15305058.2011.643517

Kruyen, P. M., Emons, W. H. M., \& Sijtsma, K. (2013). On the shortcomings of shortened tests: A literature review. International Journal of Testing, 13, 223-248. doi:10.10 80/15305058.2012.703734

Ku, K. Y. L. (2009). Assessing students' critical thinking performance: Urging for measurements using multi-response format. Thinking Skills and Creativity, 4, 70-76. doi:10.1016/j.tsc.2009.02.001

Kuijpers, R. E. (2015). Applications of categorical marginal models in test construction. 
Tilburg University.

Kuijpers, R. E., Van der Ark, L. A., \& Croon, M. A. (2013). Standard errors and confidence intervals for scalability coefficients in Mokken scale analysis using marginal models. Sociological Methodology, 43, 42-69. doi:10.1177/0081175013481958

LaHuis, D. M., \& Ferguson, M. W. (2009). The accuracy of significance tests for slope variance components in multilevel random coefficient models. Organizational Research Methods, 12, 418-435. doi:10.1177/1094428107308984

Landis, J. R., \& Koch, G. G. (1977). The measurement of observer agreement for categorical data. Biometrics, 33, 159-174. doi:10.2307/2529310

Lean Keng, S., \& AlQudah, H. N. I. (2017). Assessment of cognitive bias in decision-making and leadership styles among critical care nurses: A mixed methods study. Journal of Advanced Nursing, 73(2), 465-481. doi:10.1111/ jan. 13142

Leppa, C. J. (1997). Standardized measures of critical thinking: Experience with the california critical thinking tests. Nurse Education, 22, 29-33.

Liberali, J. M., Reyna, V. F., Furlan, S., Stein, L. M., \& Pardo, S. T. (2012). Individual differences in numeracy and cognitive reflection, with implications for biases and fallacies in probability Judgment. Journal of Behavioral Decision Making, 25, 361-381. doi:10.1002/bdm.752

Liddell, T. M., \& Kruschke, J. K. (2018). Analyzing ordinal data with metric models: What could possibly go wrong? Journal of Experimental Social Psychology, 79, 328-348. doi:10.1016/j.jesp.2018.08.009

Liu, O. L., Frankel, L., \& Roohr, K. C. (2014). Assessing critical thinking in higher education: Current state and directions for next-generation assessment. ETS Research Report Series, 2014(1), 1-23. doi:10.1002/ets2.12009

Loo, R., \& Thorpe, K. (1999). A psychometric investigation ofscores on theWatson-glaser critical thinking appraisal new forms. Educational and Psychological Measurement, 59, 995-1003.

Lorencová, H., Jarošová, E., Avgitidou, S., \& Dimitriadou, C. (2019). Critical thinking practices in teacher education programmes: A systematic review. Studies in Higher Education, 44(5), 844-859. doi:10.1080/03075079.2019.1586331

Lunn, P. D. (2013). The role of decision-making biases in Ireland's banking crisis. Irish Political Studies, 28, 563-590. doi:10.1080/07907184.2012.742068

Magidson, J., \& Vermunt, J. K. (2003). Comparing latent class factor analysis with the traditional approach in datamining. In H. Bozdogan (Ed.), Statistical data mining and knowledge discovery (pp. 373-383). Chapman \& Hall/CRC.

Marcus, B., Bosnjak, M., Lindner, S., Pilischenko, S., \& Schütz, A. (2007). Compensating for low topic interest and long surveys: A field experiment on nonresponse in web surveys. Social Science Computer Review, 25, 372-383. doi:10.1177/0894439307297606

Markovits, H., \& Nantel, G. (1989). The belief-bias effect in the production and evaluation of logical conclusions. Memory \& Cognition, 17, 11-17. doi:10.3758/BF03199552 


\section{REFERENCES}

Markovits, H., Venet, M., Janveau-Brennan, G., Malfait, N., Pion, N., \& Vadeboncoeur, I. (1996). Reasoning in young children: Fantasy and information retrieval. Child Development, 67(6), 2857. doi:10.2307/1131756

Mata, A. (2019). Conflict detection and social perception: Bringing meta-reasoning and social cognition together. Thinking \& Reasoning, 1-10. doi:10.1080/13546783.20 19.1611664

Mata, A., Ferreira, M. B., Voss, A., \& Kollei, T. (2017). Seeing the conflict: An attentional account of reasoning errors. Psychonomic Bulletin \& Review, 24, 1980-1986. doi:10.3758/s13423-017-1234-7

Mata, A., Schubert, A.-L., \& Ferreira, M. B. (2014). The role of language comprehension in reasoning: How "good-enough" representations induce biases. Cognition, 133, 457-463. doi:10.1016/j.cognition.2014.07.011

McGraw, K. O., \& Wong, S. P. (1996). Forming inferences about some intraclass correlation coefficients. Psychological Methods, 1(1), 30-46. doi:10.1037/1082989X.1.1.30

Mellenbergh, G. J. (1996). Measurement precision in test score and item response models. Psychological Methods, 1, 293-299. doi:10.1037/1082-989X.1.3.293

Mellers, B., Stone, E., Atanasov, P., Rohrbaugh, N., Metz, S. E., Ungar, L., Bishop, M. M., Horowitz, M., Merkle, E., \& Tetlock, P. (2015). The psychology of intelligence analysis: Drivers of prediction accuracy in world politics. Journal of Experimental Psychology: Applied, 21, 1-14. doi:10.1037/xap0000040

Mercier, H., \& Sperber, D. (2011). Why do humans reason? Arguments for an argumentative theory. Behavioral and Brain Sciences, 34, 57-74. doi:10.1017/ S0140525X10000968

Mevel, K., Poirel, N., Rossi, S., Cassotti, M., Simon, G., Houdé, O., \& De Neys, W. (2015). Bias detection: Response confidence evidence for conflict sensitivity in the ratio bias task. Journal of Cognitive Psychology, 27, 227-237. doi:10.1080/20445911.2 014.986487

Meyer, A., Zhou, E., \& Frederick, S. (2018). The non-effects of repeated exposure to the Cognitive Reflection Test. Judgment and Decision Making, 13, 246-259.

Misangyi, V. F., LePine, J. A., Algina, J., \& Goeddeke, F. (2006). The adequacy of repeated-measures regression for multilevel research: Comparisons with repeated-measures ANOVA, multivariate repeated-measures ANOVA, and multilevel modeling across various multilevel research designs. Organizational Research Methods, 9, 5-28. doi:10.1177/1094428105283190

Miyake, A., Friedman, N. P., Rettinger, D. A., Shah, P., \& Hegarty, M. (2001). How are visuospatial working memory, executive functioning, and spatial abilities related? A latent-variable analysis. Journal of Experimental Psychology: General, 130, 621-640. doi:10.1037//0096-3445.130.4.621

Mokken, R. J. (1971). A Theory and Procedure of Scale Analysis. De Gruyter Mouton.

Molenaar, I. W., \& Sijtsma, K. (2000). MPS5 for Windows. A program for Mokken scale analysis for polytomous items. 
Morais, F., Silva, H., Cruz, G., Pedrosa, D., Payan-Carreira, R., Dominguez, C., \& Nascimento, M. M. (2018, June 20). Perceptions of Portuguese University Teachers About Critical Thinking Educational Practices. International Conference on Technology and Innovation in Learning, Teaching and Education (TECH-EDU 2018), Thessaloniki, Greece. doi:10.1007/978-3-030-20954-4_17

National Research Council. (2012). Education for life and work: Developing transferable knowledge and skills in the 21st century (J. W. Pellegrino \& M. L. Hilton, Eds.). Board on Testing and Assessment and Board on Science Education, Division of Behavioral and Social Sciences and Education. Washington, DC: The National Academies Press.

Newman, I. R., Gibb, M., \& Thompson, V. A. (2017). Rule-based reasoning is fast and belief-based reasoning can be slow: Challenging current explanations of belief-bias and base-rate neglect. Journal of Experimental Psychology: Learning, Memory, and Cognition, 43, 1154-1170. doi:10.1037/xlm0000372

Newstead, S. E., Pollard, P., Evans, J. St. B. T., \& Allen, J. L. (1992). The source of belief bias effects in syllogistic reasoning. Cognition, 45, 257-284. doi:10.1016/00100277(92)90019-E

Nickerson, R. S. (1998). Confirmation bias: A ubiquitous phenomenon in many guises. Review of General Psychology, 2(2), 175-220. doi:10.1037/1089-2680.2.2.175

Nussbaum, M. C. (2010). Not for profit: Why democracy needs the humanities. Princeton Univ. Press.

Oster, N., \& Koesterich, R. (2013). Breaking bad behaviors: Understanding investing biases and how to overcome them. IShares Market Perspectives, 1-9.

Paas, F. (1992). Training strategies for attaining transfer of problem-solving skill in statistics: A cognitive-load approach. Journal of Educational Psychology, 84, 429-434. doi:10.1037/0022-0663.84.4.429

Paas, F., \& Van Merriënboer, J. J. G. (1993). The efficiency of instructional conditions: An approach to combine mental effort and performance measures. Human Factors: The Journal of the Human Factors and Ergonomics Society, 35, 737-743. doi:10.1177/001872089303500412

Pascarella, E. T., Blaich, C., Martin, G. L., \& Hanson, J. M. (2011). How robust are the findings of Academically Adrift? Change: The Magazine of Higher Learning, 43, 20-24. doi:10.1080/00091383.2011.568898

Paul, R. W., Elder, L., \& Bartell, T. (1997). California teacher preparation for instruction in critical thinking: Research findings and policy recommendations. California Commission on Teacher Credentialing, Sacramento.

Pennycook, G., Cheyne, J. A., Barr, N., Koehler, D. J., \& Fugelsang, J. A. (2014). Cognitive style and religiosity: The role of conflict detection. Memory \& Cognition, 42, 1-10. doi:10.3758/s13421-013-0340-7

Pennycook, G., Cheyne, J. A., Koehler, D. J., \& Fugelsang, J. A. (2015). Is the Cognitive Reflection Test a measure of both reflection and intuition? Behavior Research Methods, 48, 341-348. doi:10.3758/s13428-015-0576-1 


\section{REFERENCES}

Pennycook, G., Fugelsang, J. A., \& Koehler, D. J. (2012). Are we good at detecting conflict during reasoning? Cognition, 124, 101-106. doi:10.1016/j.cognition.2012.04.004

Pennycook, G., Fugelsang, J. A., \& Koehler, D. J. (2015a). What makes us think? A three-stage dual-process model of analytic engagement. Cognitive Psychology, 80, 34-72. doi:10.1016/j.cogpsych.2015.05.001

Pennycook, G., Fugelsang, J. A., \& Koehler, D. J. (2015b). Everyday consequences of analytic thinking. Current Directions in Psychological Science, 24, 425-432. doi:10.1177/0963721415604610

Pennycook, G., Trippas, D., Handley, S. J., \& Thompson, V. A. (2014). Base rates: Both neglected and intuitive. Journal of Experimental Psychology: Learning, Memory, and Cognition, 40, 544-554. doi:10.1037/a0034887

Perkins, D., Tishman, S., Ritchhart, R., Donis, K., \& Andrade, A. (2000). Intelligence in the wild: A dispositional view of intellectual traits. Educational Psychology Review, 12, 269-293. doi:10.1023/A:1009031605464

Pithers, R. T., \& Soden, R. (2000). Critical thinking in education: A review. Educational Research, 42, 237-249. doi:10.1080/001318800440579

Price, E., Ottati, V., Wilson, C., \& Kim, S. (2015). Open-Minded Cognition. Personality and Social Psychology Bulletin, 41, 1488-1504. doi:10.1177/0146167215600528

Primi, C., Morsanyi, K., Chiesi, F., Donati, M. A., \& Hamilton, J. (2016). The development and testing of a new version of the Cognitive Reflection Test applying Item Response Theory (IRT). Journal of Behavioral Decision Making, 29, 453-469. doi: $10.1002 /$ bdm. 1883

R Development Core Team. (2008). R: A language and environment for statistical computing. R Foundation for Statistical Computing. http://www.R-project.org.

Raoelison, M., \& De Neys, W. (2019). Do we de-bias ourselves?: The impact of repeated presentation on the bat-and-ball problem. Judgment and Decision Making, 14, $178-178$.

Reise, S. P., \& Waller, N. G. (2009). Item response theory and clinical measurement. Annual Review of Clinical Psychology, 5, 27-48. doi:10.1146/annurev. clinpsy.032408.153553

Renkl, A., \& Atkinson, R. K. (2003). Structuring the transition from example study to problem solving in cognitive skill acquisition: A cognitive load perspective. Educational Psychologist, 38, 15-22. doi:10.1207/S15326985EP3801_3

Ritchhart, R., \& Perkins, D. N. (2005). Learning to think: The challenges of teaching thinking. In K. J. Holyoak \& R. G. Morrison (Eds.), The Cambridge Handbook of Thinking and Reasoning. Cambridge University Press.

Rosseel, Y. (2012). Lavaan: An R Package for Structural Equation Modeling. Journal of Statistical Software, 48. doi:10.18637/jss.v048.i02

Sá, W. C., Kelley, C. N., Ho, C., \& Stanovich, K. E. (2005). Thinking about personal theories: Individual differences in the coordination of theory and evidence. Personality and Individual Differences, 38, 1149-1161. doi:10.1016/j. paid.2004.07.012 
Sá, W. C., \& Stanovich, K. E. (2001). The domain specificity and generality of mental contamination: Accuracy and projection in judgments of mental content. British Journal of Psychology, 92, 281-302. doi:10.1348/000712601162194

Sá, W. C., West, R. F., \& Stanovich, K. E. (1999). The domain specificity and generality of belief bias: Searching for a generalizable critical thinking skill. Journal of Educational Psychology, 91, 497-510. doi:10.1037/0022-0663.91.3.497

Schmeck, A., Opfermann, M., Van Gog, T., Paas, F., \& Leutner, D. (2015). Measuring cognitive load with subjective rating scales during problem solving: Differences between immediate and delayed ratings. Instructional Science, 43, 93-114. doi:10.1007/s11251-014-9328-3

Schmidt, H. G., Mamede, S., Van den Berge, K., Van Gog, T., Van Saase, J. L. C. M., \& Rikers, R. M. J. P. (2014). Exposure to media information about a disease can cause doctors to misdiagnose similar-looking clinical cases. Academic Medicine, 89, 285-291. doi:10.1097/ACM.0000000000000107

Schmidt, H. G., Van Gog, T., Schuit, S. C. E., Van den Berge, K., Van Daele, P. L. A., Bueving, H., Van der Zee, T., Van den Broek, W. W., Van Saase, J. L. C. M., \& Mamede, S. (2016). Do patients' disruptive behaviours influence the accuracy of a doctor's diagnosis? A randomised experiment. BMJ Quality \& Safety, 26, 19-23. doi:10.1136/bmjqs-2015-004109

Shrout, P. E., \& Fleiss, J. L. (1979). Intraclass correlations: Uses in assessing rater reliability. Psychological Bulletin, 86, 420-428. doi:10.1037/0033-2909.86.2.420

Shu, S. B., \& Townsend, C. (2014). Using aesthetics and self-affirmation to encourage openness to risky (and safe) choices. Journal of Experimental Psychology: Applied, 20, 22-39. doi:10.1037/xap0000003

Siegel, H. (1988). Educating reason: Rationality, critical thinking and education. Routledge.

Sijtsma, K., \& Molenaar, I. W. (2002). Introduction to nonparametric item response theory. SAGE.

Smith, T. C. (2017). Vaccine rejection and hesitancy: A review and call to action. Open Forum Infectious Diseases, 4(3). doi:10.1093/ofid/ofx146

Smits, I. A. M., Timmerman, M. E., \& Meijer, R. R. (2012). Exploratory Mokken scale analysis as a dimensionality assessment tool: Why scalability does not imply unidimensionality. Applied Psychological Measurement, 36(6), 516-539. doi:10.1177/0146621612451050

Spadaccini, J., \& Esteves, J. E. (2014). Intuition, analysis and reflection: An experimental study into the decision-making processes and thinking dispositions of osteopathy students. International Journal of Osteopathic Medicine, 17, 263-271. doi:10.1016/j.ijosm.2014.04.004

Šrol, J., \& De Neys, W. (2019). Predicting individual differences in conflict detection and bias susceptibility during reasoning [Preprint]. PsyArXiv. doi:10.31234/osf. io/2uf6g

Stagnaro, M., Pennycook, G., \& Rand, D. G. (2018). Performance on the Cognitive 


\section{REFERENCES}

Reflection Test is Stable across time. Judgment and Decision Making, 13, 260-267.

Stanovich, K. E. (2011). Rationality and the reflective mind. Oxford University Press.

Stanovich, K. E. (2018). Miserliness in human cognition: The interaction of detection, override and mindware. Thinking \& Reasoning, 1-22. doi:10.1080/13546783.201 8.1459314

Stanovich, K. E., \& West, R. F. (1997). Reasoning independently of prior belief and individual differences in actively open-minded thinking. Journal of Educational Psychology, 89, 342-357. doi:10.1037/0022-0663.89.2.342

Stanovich, K. E., \& West, R. F. (1998). Individual differences in rational thought. Journal of Experimental Psychology: General, 127, 161-188. doi:10.1037/00963445.127.2.161

Stanovich, K. E., \& West, R. F. (2000). Individual differences in reasoning: Implications for the rationality debate? Behavioral and Brain Sciences, 23, 645-665. doi:10.1017/ S0140525X00003435

Stanovich, K. E., \& West, R. F. (2007). Natural myside bias is independent of cognitive ability. Thinking \& Reasoning, 13, 225-247. doi:10.1080/13546780600780796

Stanovich, K. E., \& West, R. F. (2008). On the relative independence of thinking biases and cognitive ability. Journal of Personality and Social Psychology, 94, 672-695. doi:10.1037/0022-3514.94.4.672

Stanovich, K. E., West, R. F., \& Toplak, M. E. (2016). The rationality quotient: Toward a test of rational thinking. MIT Press.

Stedman, N. L. P., \& Adams, B. L. (2012). Identifying faculty's knowledge of critical thinking concepts and perceptions of critical thinking instruction in higher education. NACTA Journal, 56, 9-14.

Sternberg, R. J. (2001). Why schools should teach for wisdom: The balance theory of wisdom in educational settings. Educational Psychologist, 36, 227-245. doi:10.1207/S15326985EP3604_2

Strathman, A., Gleicher, F., Boninger, D. S., \& Edwards, C. S. (1994). The consideration of future consequences: Weighing immediate and distant outcomes of behavior. Journal of Personality and Social Psychology, 66, 742-752. doi:10.1037/00223514.66.4.742

Stupple, E. J. N., \& Ball, L. J. (2008). Belief-logic conflict resolution in syllogistic reasoning: Inspection-time evidence for a parallel-process model. Thinking \& Reasoning, 14, 168-181. doi:10.1080/13546780701739782

Stupple, E. J. N., Ball, L. J., \& Ellis, D. (2013). Matching bias in syllogistic reasoning: Evidence for a dual-process account from response times and confidence ratings. Thinking \& Reasoning, 19, 54-77. doi:10.1080/13546783.2012.735622

Sunstein, C. R. (2003). Terrorism and probability neglect. Journal of Risk and Uncertainty, 26, 121-136. doi:10.1023/A:1024111006336

Svedholm-Häkkinen, A. M., \& Lindeman, M. (2018). Actively open-minded thinking: Development of a shortened scale and disentangling attitudes towards knowledge and people. Thinking \& Reasoning, 24, 21-40. doi:10.1080/13546783.2017.13787 23 
Swami, V., Voracek, M., Stieger, S., Tran, U. S., \& Furnham, A. (2014). Analytic thinking reduces belief in conspiracy theories. Cognition, 133, 572-585. doi:10.1016/j. cognition.2014.08.006

Szaszi, B., Szollosi, A., Palfi, B., \& Aczel, B. (2017). The cognitive reflection test revisited: Exploring the ways individuals solve the test. Thinking \& Reasoning, 23, 207-234. doi:10.1080/13546783.2017.1292954

Tabachnick, B. G., \& Fidell, L. S. (2014). Using multivariate statistics (Pearson new international edition, sixth edition). Pearson.

Thibodeau, P., Peebles, M. M., Grodner, D. J., \& Durgin, F. H. (2015). The wished-for always wins until the winner was inevitable all along: Motivated reasoning and belief bias regulate emotion during elections. Political Psychology, 36, 431-448. doi:10.1111/pops. 12100

Thompson, V. A., \& Evans, J. St. B. T. (2012). Belief bias in informal reasoning. Thinking \& Reasoning, 18, 278-310. doi:10.1080/13546783.2012.670752

Thompson, V. A., \& Johnson, S. C. (2014). Conflict, metacognition, and analytic thinking. Thinking \& Reasoning, 20, 215-244. doi:10.1080/13546783.2013.869763

Thompson, V. A., Pennycook, G., Trippas, D., \& Evans, J. St. B. T. (2018). Do smart people have better intuitions? Journal of Experimental Psychology: General, 147, 945-961. doi:10.1037/xge0000457

Thompson, V. A., Prowse Turner, J. A., \& Pennycook, G. (2011). Intuition, reason, and metacognition. Cognitive Psychology, 63, 107-140. doi:10.1016/j. cogpsych.2011.06.001

Thompson, V. A., Turner, J. A. P., Pennycook, G., Ball, L. J., Brack, H., Ophir, Y., \& Ackerman, R. (2013). The role of answer fluency and perceptual fluency as metacognitive cues for initiating analytic thinking. Cognition, 128, 237-251. doi:10.1016/j.cognition.2012.09.012

Thompson, W. C., \& Schumann, E. L. (1987). Interpretation of statistical evidence in criminal trials: The prosecutor's fallacy and the defense attorney's fallacy. Law and Human Behavior, 11, 167-187. doi:10.1007/BF01044641

Thomson, K. S., \& Oppenheimer, D. M. (2016). Investigating an alternate form of the Cognitive Reflection Test. Judgment and Decision Making, 11, 99-113.

Tishman, S., Jay, E., \& Perkins, D. N. (1993). Teaching Thinking Dispositions:

From Transmission to Enculturation. Theory Into Practice, 32, 147-153. doi:10.1080/00405849309543590

Toplak, M. E., \& Stanovich, K. E. (2002). The domain specificity and generality of disjunctive reasoning: Searching for a generalizable critical thinking skill. Journal of Educational Psychology, 94, 197-209. doi:10.1037//0022-0663.94.1.197

Toplak, M. E., West, R. F., \& Stanovich, K. E. (2011). The Cognitive Reflection Test as a predictor of performance on heuristics-and-biases tasks. Memory \& Cognition, 39, 1275-1289. doi:10.3758/s13421-011-0104-1

Toplak, M. E., West, R. F., \& Stanovich, K. E. (2014a). Assessing miserly information processing: An expansion of the Cognitive Reflection Test. Thinking \& Reasoning, 20, 147-168. doi:10.1080/13546783.2013.844729

Toplak, M. E., West, R. F., \& Stanovich, K. E. (2014b). Rational thinking and cognitive 
sophistication: Development, cognitive abilities, and thinking dispositions. Developmental Psychology, 50, 1037-1048. doi:10.1037/a0034910

Toplak, M. E., West, R. F., \& Stanovich, K. E. (2017). Real-World Correlates of Performance on Heuristics and Biases Tasks in a Community Sample: Heuristics and Biases Tasks and Outcomes. Journal of Behavioral Decision Making, 30, 541-554. doi:10.1002/bdm. 1973

Travers, E., Rolison, J. J., \& Feeney, A. (2016). The time course of conflict on the Cognitive Reflection Test. Cognition, 150, 109-118. doi:10.1016/j. cognition.2016.01.015

Trouche, E., Johansson, P., Hall, L., \& Mercier, H. (2016). The selective laziness of reasoning. Cognitive Science, 40, 2122-2136. doi:10.1111/cogs.12303

Tversky, A., \& Kahneman, D. (1974). Judgment under uncertainty: Heuristics and biases. Science, 185, 1124-1131. doi:10.1126/science.185.4157.1124

Tversky, A., \& Kahneman, D. (1983). Extensional Versus Intuitive Reasoning: The Conjunction Fallacy in Probability Judgment. Psychological Review, 90, 293-315. doi:10.1016/B978-1-4832-1446-7.50038-8

Tversky, A., \& Kahneman, D. (1986). Rational choice and the framing of decisions. The Journal of Business, 59, S251-S278. doi:10.1086/296365

Van Aalderen-Smeets, S. I., \& Walma van der Molen, J. H. (2013). Measuring primary teachers' attitudes toward teaching science: Development of the Dimensions of Attitude toward Science (DAS) instrument. International Journal of Science Education, 35, 577-600. doi:10.1080/09500693.2012.755576

Van Aalderen-Smeets, S. I., \& Walma van der Molen, J. H. (2015). Improving primary teachers' attitudes toward science by attitude-focused professional development. Journal of Research in Science Teaching, 52, 710-734. doi:10.1002/tea.21218

Van den Berge, K., Mamede, S., Van Gog, T., Romijn, J. A., Van Guldener, C., Van Saase, J. L., \& Rikers, R. M. (2012). Accepting diagnostic suggestions by residents: A potential cause of diagnostic error in medicine. Teaching and Learning in Medicine, 24, 149-154. doi:10.1080/10401334.2012.664970

Van der Ark, L. A. (2007). Mokken scale analysis in R. Journal of Statistical Software, 20, $1-19$.

Van der Ark, L. Andries, Van der Palm, D. W., \& Sijtsma, K. (2011). A latent class approach to estimating test-score reliability. Applied Psychological Measurement, 35, 380-392. doi:10.1177/0146621610392911

Van der Palm, D. W., Van der Ark, L. A., \& Sijtsma, K. (2014). A flexible latent class approach to estimating test-score reliability. Journal of Educational Measurement, 51, 339-357. doi:10.1111/jedm.12053

Van Gog, T., Kirschner, F., Kester, L., \& Paas, F. (2012). Timing and frequency of mental effort measurement: Evidence in favour of repeated measures. Applied Cognitive Psychology, 26, 833-839. doi:10.1002/acp.2883

Van Gog, T., \& Paas, F. (2008). Instructional efficiency: Revisiting the original construct in educational research. Educational Psychologist, 43, 16-26. doi:10.1080/00461520701756248 
Van Gog, T., Rummel, N., \& Renkl, A. (2019). Learning how to solve problems by studying examples. In J. Dunlosky \& K. A. Rawson (Eds.), The Cambridge Handbook of Cognition and Education (1st ed., pp. 183-208). Cambridge University Press. doi:10.1017/9781108235631.009

Van Kelecom, K. (2017, February). We zeggen te vaak sorry (én we doen het verkeerd) [Taalblad.be]. https://web.archive.org/web/20181209123856/http:/taalblad. be/e-zine/kort-en-klein/we-zeggen-te-vaak-sorry-en-we-doen-het-verkeerd-/1314. html

Van Peppen, L. M., Verkoeijen, P. P. J. L., Heijltjes, A. E. G., Janssen, E. M., Koopmans, D., \& Van Gog, T. (2018). Effects of self-explaining on learning and transfer of critical thinking skills. Frontiers in Education, 3. doi:10.3389/feduc.2018.00100

Van Veen, K., Zwart, R., \& Meirink, J. (2012). What makes teacher professional development effective? A literature review. In M. Kooy \& K. Van Veen (Eds.), Teacher learning that matters: International perspectives (1st ed., pp. 23-41). Routledge. doi:10.4324/9780203805879

Venet, M., \& Markovits, H. (2001). Understanding uncertainty with abstract conditional premises. Merrill-Palmer Quarterly, 47, 74-99. doi:10.1353/mpq.2001.0006

Wason, P. C. (1968). Reasoning about a rule. Quarterly Journal of Experimental Psychology, 20, 273-281. doi:10.1080/14640746808400161

Wasserman, E. A., Dorner, W. W., \& Kao, S. F. (1990). Contributions of specific cell information to judgments of interevent contingency. Journal of Experimental Psychology: Learning, Memory, and Cognition, 16, 509-521. doi:10.1037/02787393.16.3.509

Watt, H. M. G., \& Richardson, P. W. (2007). Motivational factors influencing teaching as a career choice: Development and validation of the FIT-choice scale. The Journal of Experimental Education, 75(3), 167-202. doi:10.3200/JEXE.75.3.167-202

West, R. F., Meserve, R. J., \& Stanovich, K. E. (2012). Cognitive sophistication does not attenuate the bias blind spot. Journal of Personality and Social Psychology, 103, 506-519. doi:10.1037/a0028857

West, R. F., Toplak, M. E., \& Stanovich, K. E. (2008). Heuristics and biases as measures of critical thinking: Associations with cognitive ability and thinking dispositions. Journal of Educational Psychology, 100, 930-941. doi:10.1037/a0012842

Zee, M., \& Koomen, H. M. Y. (2016). Teacher self-efficacy and its effects on classroom processes, student academic adjustment, and teacher well-being: A synthesis of 40 years of research. Review of Educational Research, 86, 981-1015. doi:10.3102/0034654315626801

Zizzo, D. J., Stolarz-Fantino, S., Wen, J., \& Fantino, E. (2000). A violation of the monotonicity axiom: Experimental evidence on the conjunction fallacy. Journal of Economic Behavior \& Organization, 41, 263-276. doi:10.1016/ S0167-2681(99)00076-1 
DANKWOORD 
Met veel plezier en enthousiasme heb ik aan dit proefschrift gewerkt. De vrijheid die ik kreeg, de afwisseling van werkzaamheden, het samenwerken, het alleen ergens in vastbijten, ik vond het allemaal leuk. Echter, zo heel af en toe, maakten de positieve emoties ook wel eens plaats voor ietwat minder aangename gevoelens, variërend van lichte twijfels ("Had ik niet toch...?") tot existentiële crises ("Als ik morgen onder een bus kom, zou dan überhaupt één iemand zich nog afvragen wat er uit dit onderzoek is gekomen?”). Mensen die mij wat beter kennen, zullen niet verrast zijn dat ik zowel de positieve als de negatieve emoties doorgaans vrij intens beleefde. Dat ik terugkijk op een intens leuk en leerzaam traject, waarin de minder leuke ervaringen altijd weer vrij snel naar de achtergrond verdwenen, komt door de onmisbare hulp die ik uit zo veel verschillende hoeken ontving, de belangstelling die mensen voor mijn onderzoek toonden, de zeer welkome afleiding die vaak op mijn pad kwam en de liefde en steun waar ik te allen tijde op kon rekenen.

De onmisbare hulp bij mijn onderzoek ontving ik in de eerste plaats van mijn projectteam. Tamara, Tim, Peter, Anita en Lara, bedankt voor de warme samenwerking. Wat fijn om in een team te werken met zo'n diversiteit aan expertise, waarin iedereen zich voor de volle honderd procent inzet, maar waarin ook ruimte is voor relativering en een grapje. Tamara, bedankt voor al je vindingrijke oplossingen, het openstaan voor mijn eigen ideeën, het stimuleren om deze ideeën daadwerkelijk uit te voeren en het leggen van de hoge lat bij elke studie. Tim, bedankt voor je kritische bevragingen, je aanhoudende aanmoedigingen om mijn argumenten strakker te verwoorden en je aanstekelijke enthousiasme wanneer het statistiek betrof. Peter, bedankt voor je geduld, je motiverende feedback en het vertrouwen dat je me gaf in mijn eigen capaciteiten. Dankzij jou begrijp ik meer van de psychometrie dan ik ooit had durven hopen. Anita, wat vond ik het spannend om, vooral die eerste keer, docenttrainingen te geven, maar wat voelde ik me gesterkt door jouw hulp, aanwezigheid en vertrouwen. Ik heb veel geleerd van jouw expertise als trainer, jouw kennis en vaardigheid als onderzoeker en van jouw drive om onderwijspraktijk en onderzoek met elkaar te verbinden. Lara, fijn om tegelijk met jou aan dit traject te beginnen, om de symposia en workshops met jou te organiseren, om een hotelkamer met jou te delen tijdens de congressen en om onze succesjes samen te vieren. Ik denk dat we elkaar goed aanvulden.

Collega's van de afdeling Educatie, bedankt voor het delen van jullie kennis, ervaringen en expertise. Jullie gaven me allemaal het gevoel altijd binnen te mogen lopen voor een vraag of advies. In het bijzonder Steven, zonder onze koffieautomaatoverleggen was mijn onderzoek niet hetzelfde geweest. Luce, fijn dat jij me altijd zo goed begrijpt en voor elk probleem een praktische oplossing hebt. Jos, wat een geluk had ik dat ik altijd op jouw, uiterst vriendelijke, technische ondersteuning kon rekenen, bedankt! Alle studenten en onderzoeksassistenten die me hebben geholpen, Wietse, Samuël, Anne-Roos, Aitana, Marja, dankjewel (thank you)!

Alle collega's van het lectoraat Brein en Leren van Avans, bedankt voor de inspirerende kenniskringbijeenkomsten, de interesse, het meeleven en voor de onmisbare hulp bij mijn dataverzamelingen. In het bijzonder Stefan, zonder jouw enthousiaste initiatief en ondersteuning was de tweede trainingsstudie er niet geweest. Milou, in planning en organisatie zal ik nooit uitblinken, maar ik heb wel geleerd van jouw skills op dit vlak, dankjewel! Marion, bedankt voor de fijne samenwerking bij het geven van onze workshop in Groningen. Stefan, Milou, Marion, Hans, Janneke, Anton, Marloes, Yvonne en Ilse, bedankt voor jullie bereidheid om niet alleen zelf, maar ook jullie collega's te vragen om bij te dragen aan mijn 
onderzoek. Peter en Anita, wat leiden jullie een fantastisch lectoraat!

To all colleagues at the LaPsyDé in Paris, thank you for making my visit a great experience. Malheureusement, mon français n'est pas asses bon pour boire un verre en français le vendredi. Wim, thank you for your willingness to supervise me, for your calm and open mind, and for sharing your expertise. I learnt so much in those three months. Matthieu, thank you for the nice collaboration, especially for the "live" $\mathrm{R}$ programming sessions.

Alle 2.144 deelnemers aan mijn onderzoek, zonder jullie tijd en moeite was dit onderzoek nergens, dank jullie wel. In het bijzonder de docenten op Avans die de intensieve kritisch-denken testen maakten en/of deelnamen aan mijn training. Bedankt voor jullie tijd, aandacht, nieuwsgierigheid en eerlijkheid. Ik heb veel van jullie geleerd, vooral hoe divers de groep docenten is die achter mijn gerapporteerde statistieken schuilt.

Mijn kritische familieleden en vrienden, papa, mama, Maria, Anouk, Jasmijn (en vrienden), Marieke, Jochanan, Sara (en vrienden), Sanne, Roel, Brady (en collega's), dank jullie wel dat jullie steeds opnieuw bereid waren om de door mij ontworpen testtaken uit te proberen. Jullie scoorden vaak zo hoog dat ik onterecht dacht dat de taken te makkelijk waren. Renate, bedankt voor je hulp bij mijn statistische analyses en je aanmoediging om op R over te stappen. Sara, bedankt voor je creativiteit en voor het opvrolijken van de werkdagen waarin ik samen met jou aan onderzoeksmaterialen kon werken. Anouk, bedankt voor het delen van je docentexpertise. Ik heb veel geleerd van die ene keer dat jij mijn trainingsslides onder handen nam. Hakim, bedankt voor het strakke design van de voorkant en lay-out van dit proefschrift, vooral bedankt dat je het zo vanzelfsprekend vond om dit voor mij te doen.

Naast de directe hulp bij het uitvoeren van mijn onderzoek, zijn er zoveel mensen die op een andere manier hebben bijgedragen aan een succesvolle afronding van dit proefschrift. Graag wil ik iedereen bedanken die zo oprecht geïnteresseerd is geweest in mijn onderzoek, een grotere motivator bestaat niet, en die mij zeer welkome afleiding heeft geboden of die me simpelweg het gevoel heeft gegeven goed genoeg te zijn.

Ook hiervoor bedank ik al mijn collega's. De positieve sfeer op onze afdeling heeft veel bijgedragen aan mijn werkplezier. Steven, fijn om iemand te hebben gevonden die het ook echt leuk vindt om heel lang door te praten over werkelijk waar elk thema dat door een van ons wordt aangedragen. Luce, ik ben me altijd een beetje jouw minion blijven voelen, zo'n goed gevoel is dat. Bas, bedankt voor jouw vrolijkheid en provocerende grappen. Ik hoop dat we onze congreskroegentochten blijven voortzetten en daarbij natuurlijk ook onze karaokeprestaties blijven neerzetten. Sophie, bedankt voor onze ontelbare Guti's. Het uitwisselen van ups en downs is toch het leukst met iemand met een vergelijkbare emotionele aard. Loes, ik voelde direct dat het goed zat tussen ons. Carolien(tje), ik heb veel geleerd van de nuchtere, humoristische en positieve blik waarmee jij naar de dingen kijkt. Milou, bedankt voor je luisterend oor en je ontwapenende verwondering. Tim, bedankt dat ik zo snel bij jou en Steven mocht aanschuiven op nasi-donderdag en voor de droge, soms wat cynische, levenslessen die je mij meegaf op onze kamer of tijdens een van de speciaalbiertjes. Lara, congressen en samenwerken vond ik extra leuk door onze gesprekken die even niet over onderzoek gingen, doorgaans in leukste horeca dankzij jouw uitgebreide vooronderzoek. Rob, bedankt voor jouw kwetsbare eerlijkheid en tomeloos enthousiasme. Esther, Monika, Minke, bedankt voor de veel te lange, veel te interessante kamergesprekken over zeer uiteenlopende 
onderwerpen. Tamara, jouw pogingen om mij aan het hardlopen te krijgen waren niet altijd even succesvol, maar aan die ene geslaagde poging, de Bruggenloop, heb ik nog steeds goede herinneringen. Tim, de enerverende gesprekken op de feestjes na werk heb ik altijd als een zeer aangename afwisseling ervaren... Peter, al jouw anekdotes, bijvoorbeeld over geërfde boekjes van Duitse ooms ("1x1 des guten Tons"), toverden altijd direct een hele grote lach op mijn gezicht. Anita, wat een fijne herinneringen heb ik aan ons gezamenlijke bezoek aan de OECD-bijeenkomst in Parijs; ik vond het zo gezellig. E3.36 (oud)kamergenoten Alex, Bas, Esther, Jaël, Joris, Michelle, Minke, Monika, Steven, Tim, bedankt voor het bieden van een veilige thuishaven op werk. Caroline, werken voor ICO was niet alleen leerzaam, maar ook gewoon erg leuk omdat ik dan weer reden had om even bij jou langs te lopen voor een overleg en een praatje. Margot, bedankt voor onze korte maar warme samenwerking. Vincent, bedankt voor je aanmoedigende woorden op de juiste momenten. Martine, leuk om met jou ervaringen uit te wisselen, hopelijk blijven we dat doen. Bjorn, bedankt voor je interesse en goocheltrucs.

Daarnaast wil ik graag mijn vrienden bedanken, die mij met hun kennis, luisterend oor, humor, afleiding en liefde altijd weer tot nieuwe inzichten brengen en mij vooral doen beseffen dat werk niet het belangrijkste is. In het bijzonder Anouk, Jasmijn, Marieke en Simona, samen met jullie zijn voelt als thuiskomen. Esther en Nena, we zijn alle drie anders maar op de belangrijke dingen zo hetzelfde. Sanne en Sara, na het daverende succes van onze onvoorbereide fietsvakantie op barrels van fietsen wist ik dat alles met jullie leuk is. Renate, jouw vertrouwen in mijn capaciteiten heeft me zoveel gebracht.

Tot slot, maar verre van 'at least', bedank ik mijn lieve familie. Papa en mama, fijn dat jullie me zo mijn eigen gang (hebben) laten gaan en me steunen waar jullie kunnen. Papa, bedankt voor je relativerende opmerkingen die me met beide benen op de grond houden, zoals "Ik vind het niet zo'n verrassende uitkomst, had je daar zo'n lang onderzoek voor nodig?" Hoewel ik vermoed dat je het ook gewoon wel leuk vindt om te zeggen, blijf je me uitdagen na te denken over het nut van (mijn) onderzoek. Mama, jij kan mensen zo'n fijn, geliefd gevoel geven. Je hebt mij altijd het gevoel hebt gegeven dat ik in jouw ogen meer dan goed genoeg ben, dankjewel. Als kind al genoot ik er zo van om met jou over van alles en nog wat te kletsen, dat doe ik nu nog steeds. Simone, voor altijd mijn grote zus, bedankt voor jouw eerlijkheid, je eigenzinnigheid en je grootse liefde voor familie. Ik heb een hoop geleerd van je geleerd. Peyman, wat bewonder ik jouw geduldige, ruimdenkende en leergierige karakter. Sasha, drie dagen voor het inleveren van dit proefschrift maakte je mij tot trotse tante van jou. Ik hoop dat je jouw ereplekje in de Nederlandse samenvatting kan waarderen later. Tante Froukje, oom Arnold, Rosa en Maria, ik ben zo blij dat jullie er zijn. Opa, jouw onbegrensde belangstelling, nieuwsgierigheid en verwondering inspireren mij nog steeds. Wat had ik graag je trotse gezicht in de zaal zien zitten .

Lieve Brady, ik hoop dat je altijd zo nieuwsgierig, grappig, veerkrachtig en zelfverzekerd blijft. Ik vind het leven zo leuk zo samen met jou. 
CURRICULUM VITAE AND PUBLICATIONS 
Eva Janssen was born on October 24th 1990 in Delft (the Netherlands). She completed her secondary education in 2009 at the Stanislas College in Delft. In 2013, she graduated from the Master Youth, Education and Society at Utrecht University (cum laude). Hereafter, she applied for the Research Master Educational Sciences: Learning in Interaction at the same university and graduated in 2015 (cum laude). In 2016, Eva started her PhD at Utrecht University. Her $\mathrm{PhD}$ research was part of a larger project. In this project, researchers from Utrecht University and Erasmus University Rotterdam collaborated closely with educational advisors, teachers, and researchers from Avans University of Applied Sciences. Together they investigated how to improve and maintain students' critical thinking skills to avoid bias in reasoning and to achieve transfer, and how to equip teachers for teaching these skills to their students. In January 2019, Eva spent three months at the Université Paris-Descartes (Paris-V; part of Université Sorbonne Paris Cité). She presented her work at various (inter)national conferences (e.g., ICO, ORD, EARLI, AERA). She was a member of the Educational Committee of the national research school, the Interuniversity Center for Educational Sciences (ICO). She was also PhD coordinator for the ICO theme group Learning and Instruction. Eva is currently employed as a postdoctoral researcher at the Department of Education at Utrecht University.

\section{Publications (reverse chronological):}

Janssen, E. M., Verkoeijen, P. P. J. L., Heijltjes, A. E. G., Mainhard, T., Van Peppen, L. M., \& Van Gog, T. (2020). Psychometric properties of the Actively Open-minded Thinking scale. Thinking Skills and Creativity, 36, 100659. doi:10.1016/j.tsc.2020.100659

Janssen, E.M., Raoelison, M., \& De Neys, W. (2020). "You're wrong!": The impact of accuracy feedback on the bat-and-ball problem. Acta Psychologica, 206, 103042. doi:10.1016/j. actpsy.2020.103042

Janssen, E. M., Mainhard, T., Buisman, R. S. M., Verkoeijen, P. P. J. L., Heijltjes, A. E. G., Van Peppen, L. M., \& Van Gog, T. (2019). Training higher education teachers' critical thinking and attitudes towards teaching it. Contemporary Educational Psychology, 58, 310-322. doi:10.1016/j.cedpsych.2019.03.007

Janssen, E. M., Meulendijks, W., Mainhard, T., Verkoeijen, P. P. J. L., Heijltjes, A. E. G., Van Peppen, L. M., \& Van Gog, T. (2019). Identifying characteristics associated with higher education teachers' Cognitive Reflection Test performance and their attitudes towards teaching critical thinking. Teaching and Teacher Education, 84, 139-149. doi:10.1016/j. tate.2019.05.008

Van Peppen, L. M., Verkoeijen, P. P. J. L., Heijltjes, A. E. G., Janssen, E. M., Koopmans, D., \& Van Gog, T. (2018). Effects of self-explaining on learning and transfer of critical thinking skills. Frontiers in Education, 3. doi:10.3389/feduc.2018.00100

Janssen, E. M., Van der Ven, S. H. G., Van Hoogmoed, A. H., \& Leseman, P. P. M. (2017). The effect of anticipated achievement feedback on students' semantic processing as indicated by the N400 cloze effect. Learning and Instruction, 47, 80-90. doi:10.1016/j.learninstruc.2016.10.003

Van der Ven, S. H. G., Van Touw, S. A. C., Van Hoogmoed, A. H., Janssen, E. M., \& Leseman, P. P. M. (2017). The effect of a prospected reward on semantic processing: An N400 EEG study. Zeitschrift für Psychologie, 224, 257-265. doi:10.1027/2151-2604/a000261 



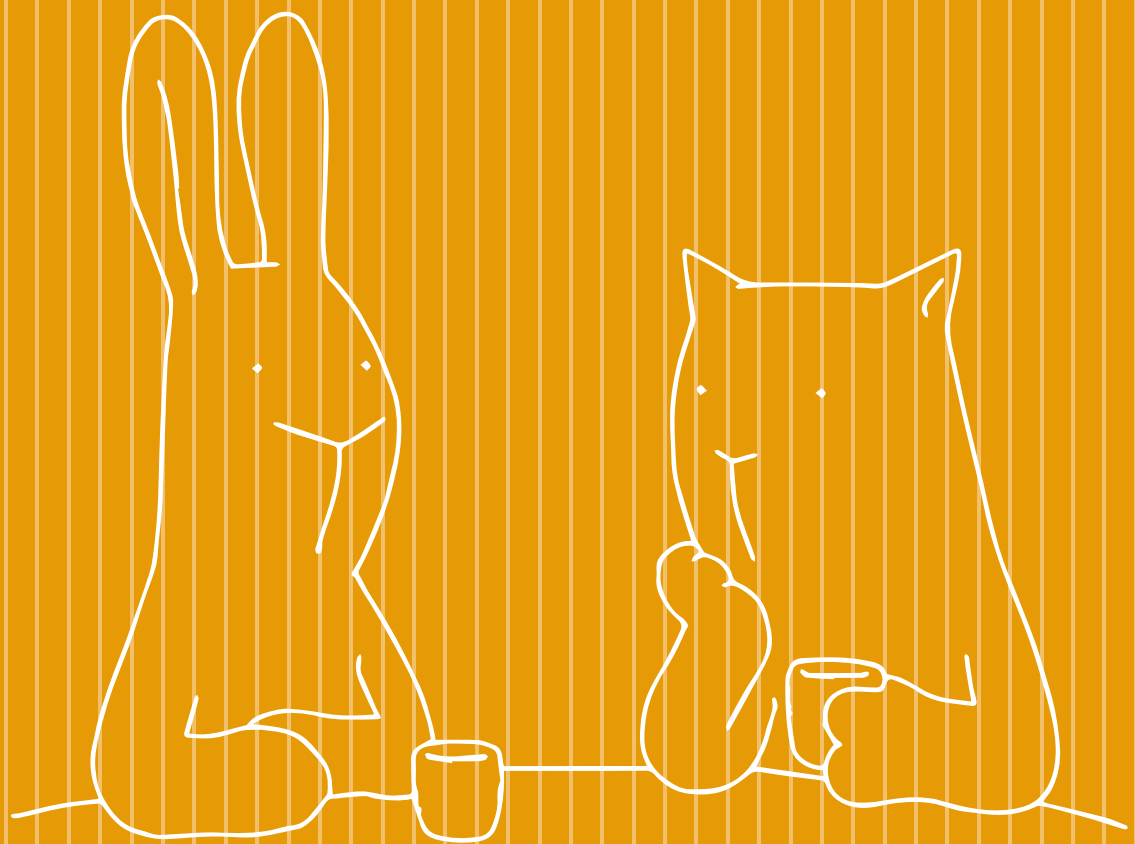

Ik begin steeds meer te begrijpen dot ik heel veel dingen niet snop. 